NATIONAL LABORATORY

\title{
Supply Chain-Based Solution to Prevent Fuel Tax Evasion: Proof of Concept Final Report
}

\section{November 2011}

Prepared by

Gary Capps

Mary Beth Lascurain

Oscar Franzese

Duncan Earl

David West

Timothy McIntyre

Shih-Miao Chin

Ho-Ling Hwang

Raynella Connatser

Samuel Lewis

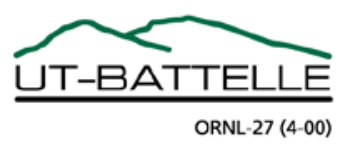





\title{
SUPPLY CHAIN-BASED SOLUTION TO PREVENT FUEL TAX EVASION: PROOF OF CONCEPT FINAL REPORT
}

\author{
Gary Capps \\ Mary Beth Lascurain \\ Oscar Franzese \\ Duncan Earl \\ David West \\ Timothy McIntyre \\ Shih-Miao Chin \\ Ho-Ling Hwang \\ Raynella Connatser \\ Samuel Lewis
}

Date Published: November 2011

\author{
Prepared by \\ OAK RIDGE NATIONAL LABORATORY \\ Oak Ridge, Tennessee 37831-6283 \\ managed by \\ UT-BATTELLE, LLC \\ for the \\ U.S. DEPARTMENT OF ENERGY \\ under contract DE-AC05-00OR22725
}





\section{CONTENTS}

\section{Page}

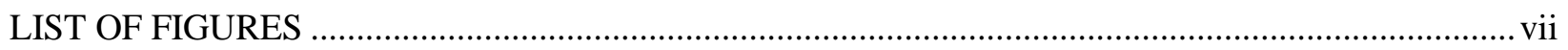

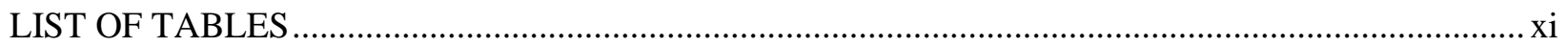

ACRONYMNS AND ABBREVIATIONS ..................................................................................

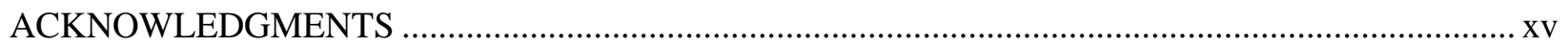

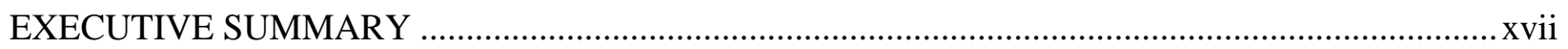

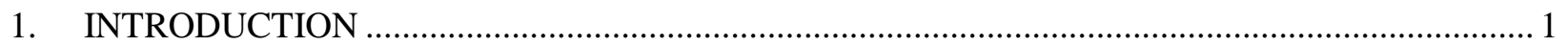

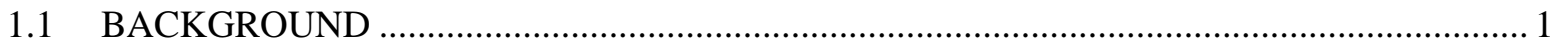

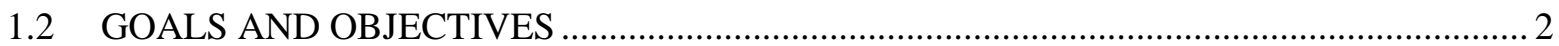

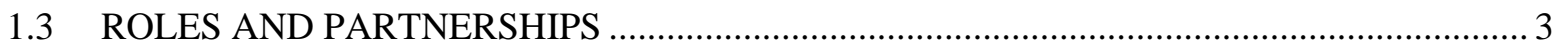

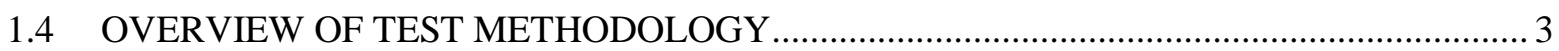

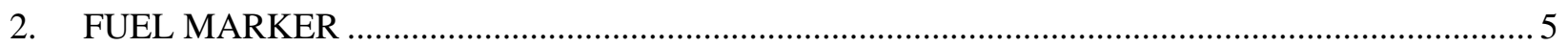

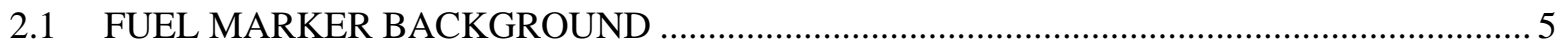

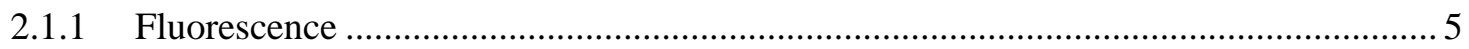

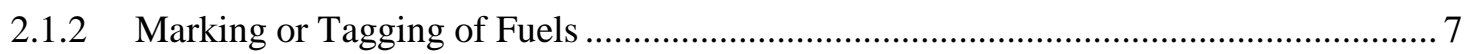

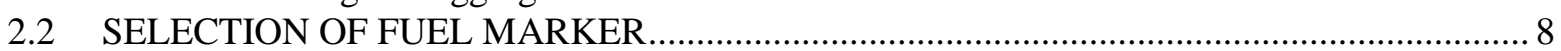

2.2.1 Required Marker Characteristics: Compatibility as a Fuel Additive ........................... 10

2.2.2 Required Marker Characteristics: Utility as a Tracking Moiety ............................... 11

2.3 AGING AND CHEMICAL STABILITY TESTING ................................................... 17

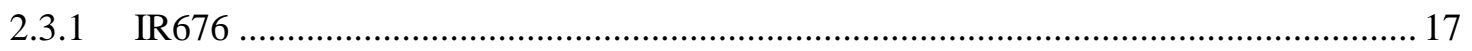

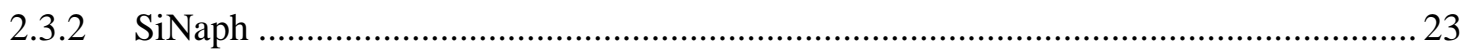

2.3.3 Sister Dye: 3,3'-diethylthiatricarbocyanine iodide ................................................. 27

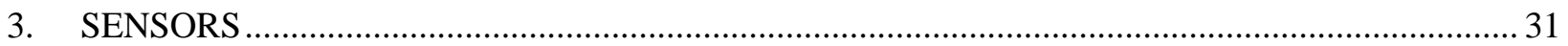

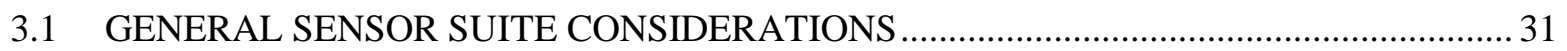

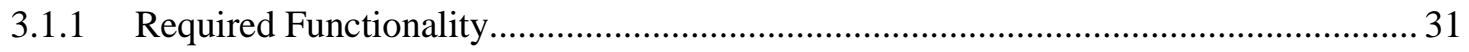

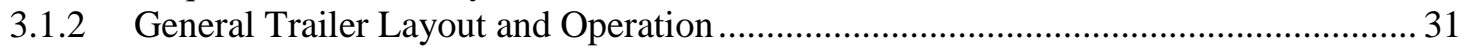

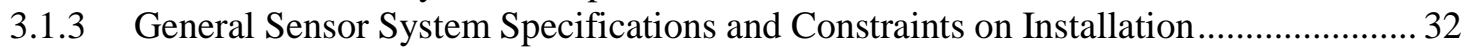

3.2 HATCH AND VALVE SENSOR DEVELOPMENT AND EVALUATION ......................... 33

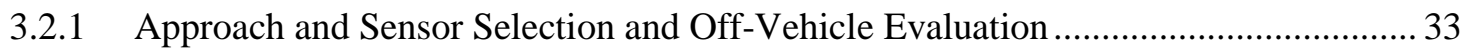

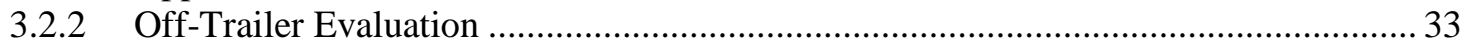

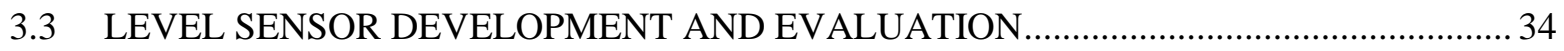

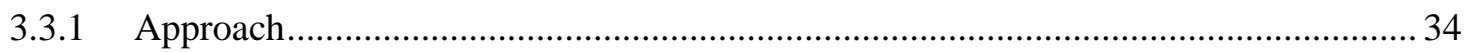

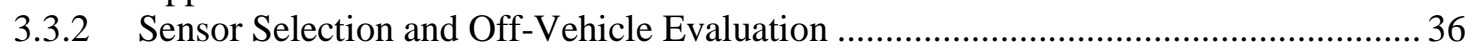

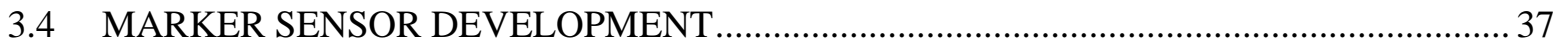

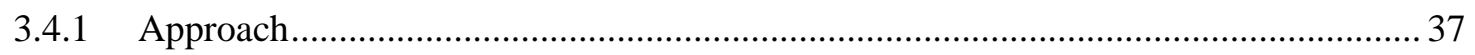

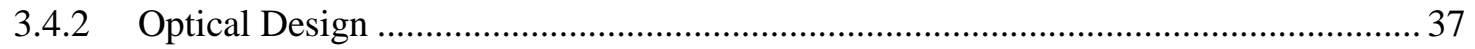

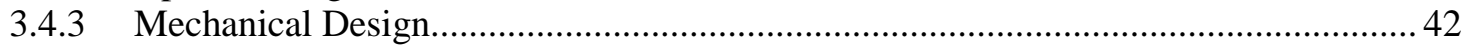

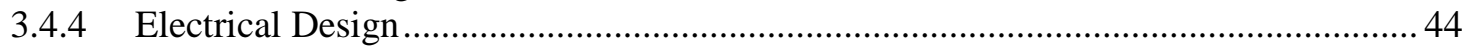

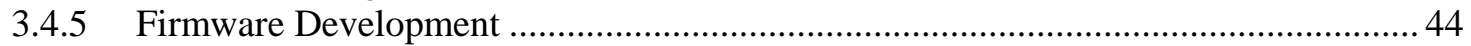

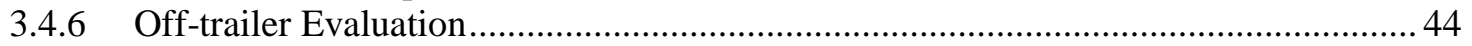


3.5 SENSOR SYSTEM LAYOUT AND CONFIGURATION ............................................ 47

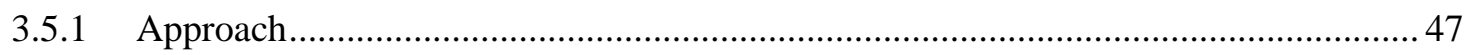

3.5.2 Off-Trailer Evaluation and Development ............................................................ 47

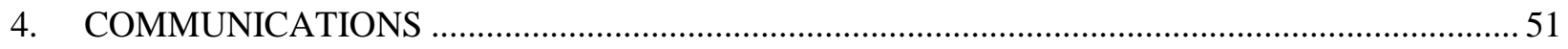

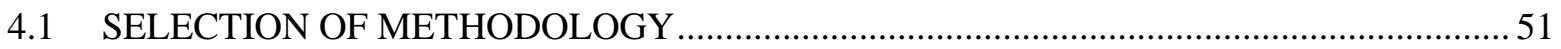

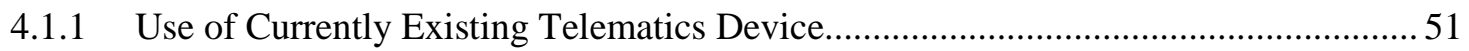

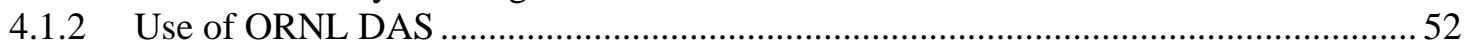

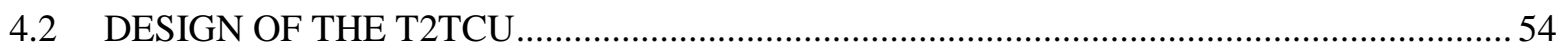

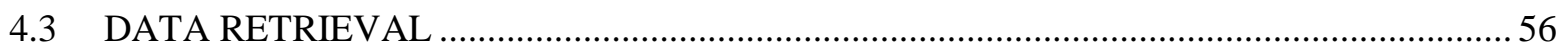

5. GEO-FENCING AND EVIDENTIAL REASONING APPLICATIONS .......................................... 59

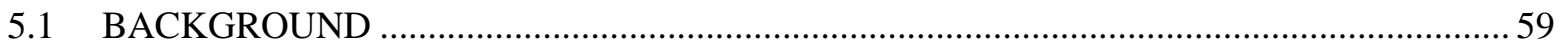

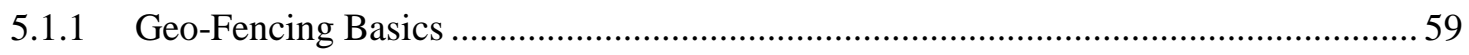

5.1.2 Fundamentals of Evidential Reasoning Methods......................................................... 59

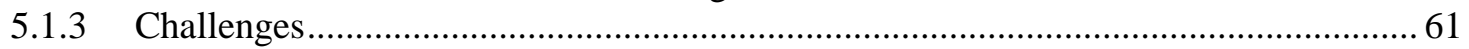

5.2 REAL-WORLD FUEL DELIVERY OPERATION DATA COLLECTION EFFORTS.......... 62

5.2.1 Real-World Information Relating to Pilot Tanker Truck Operations ........................... 62

5.2.2 Truck Travel Behavior Data (PeopleNet Fleet Manger Reports) ...................................66

5.2.3 Service Station, Terminals, and Wholesale Customer Locations .................................. 68

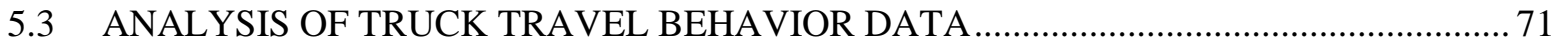

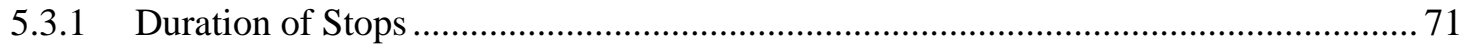

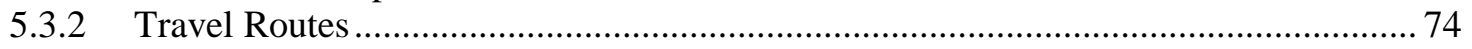

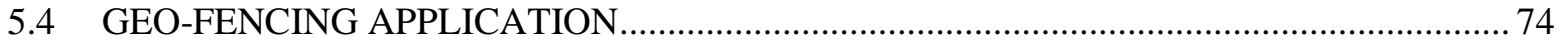

5.4.1 Methodologies to Detect Out-of-Fence Travel Behavior............................................ 74

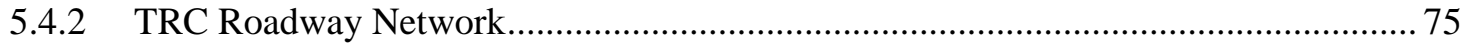

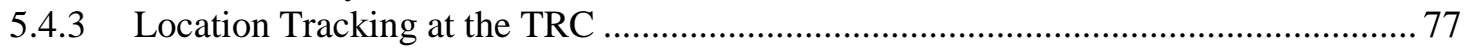

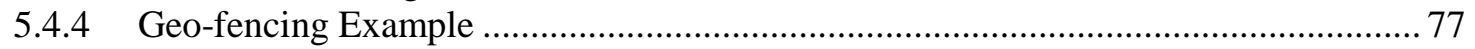

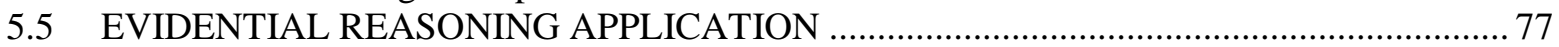

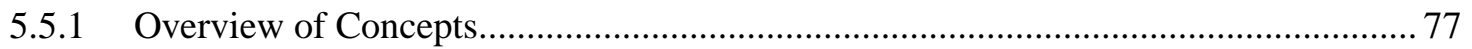

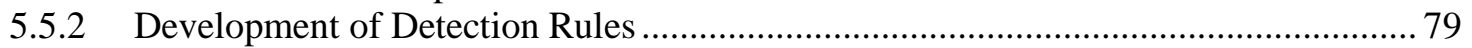

5.5.3 Determining Uncertainties in Marker Concentration and Fuel Level

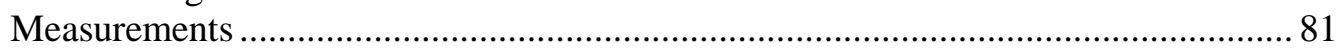

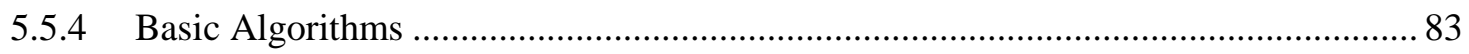

5.6 INTEGRATING THE GEO-FENCING AND EVIDENTIAL REASONING RULES ............ 84

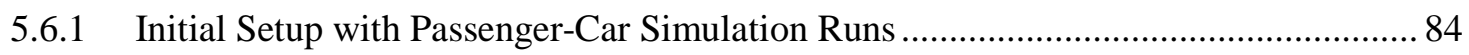

5.6.2 Additional Passenger Car Simulation Scenarios on Actual Truck Routes.................... 84

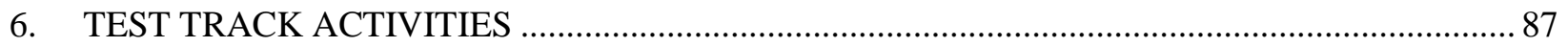

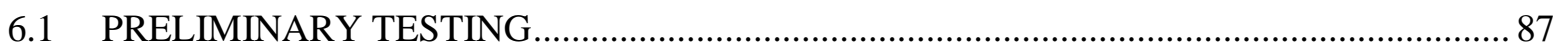

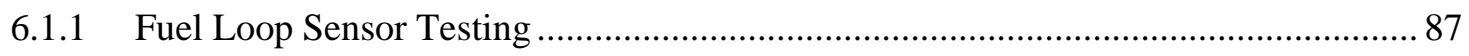

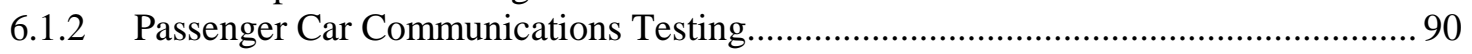

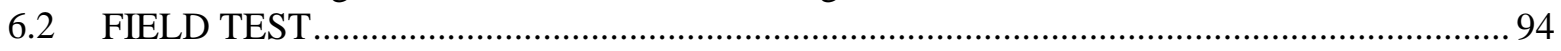

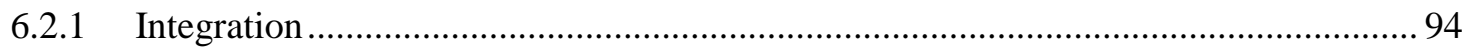

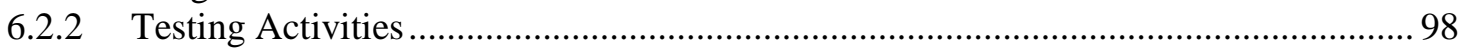

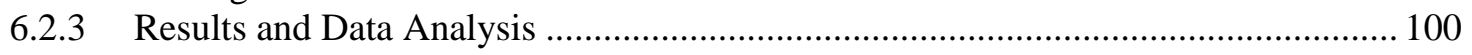

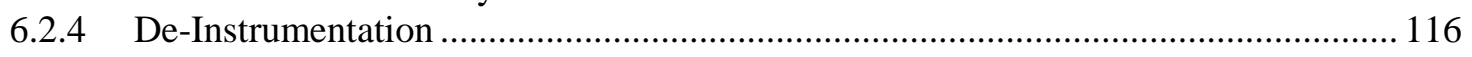

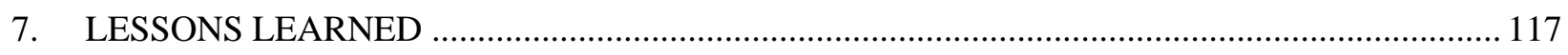

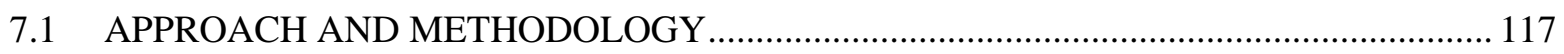

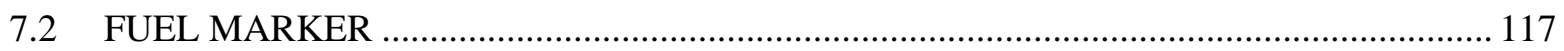




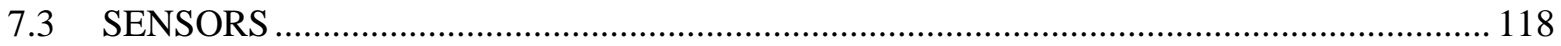

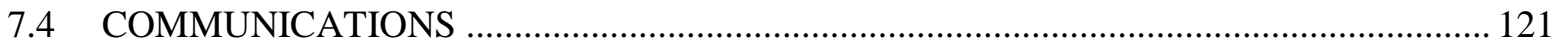

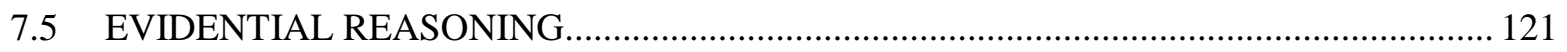

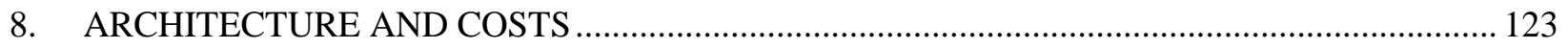

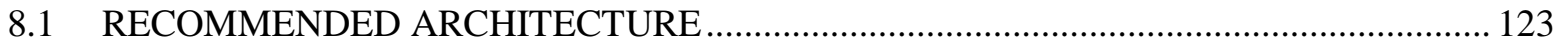

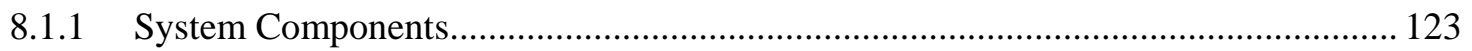

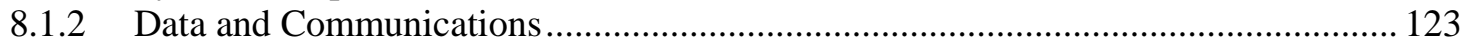

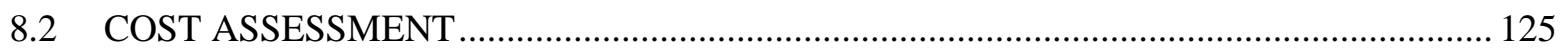

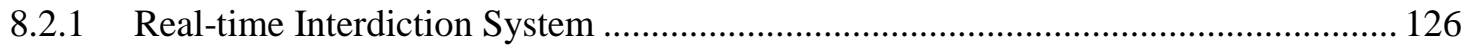

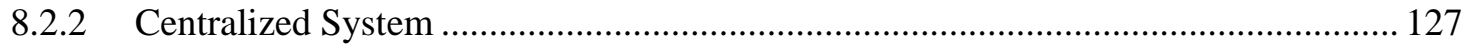

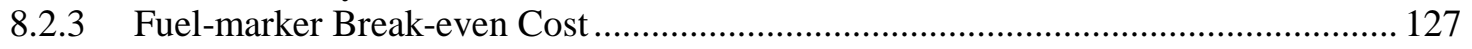

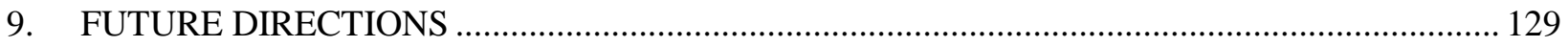

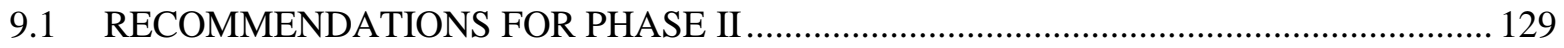

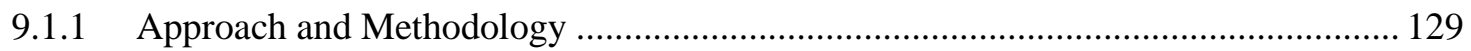

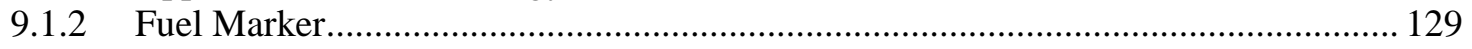

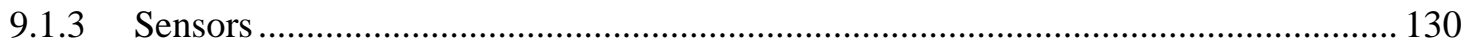

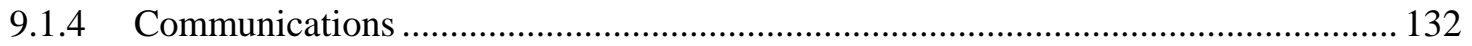

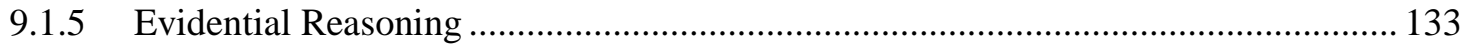

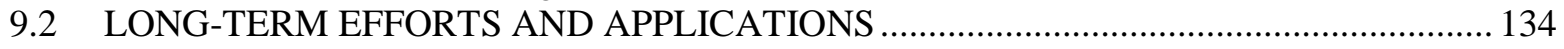

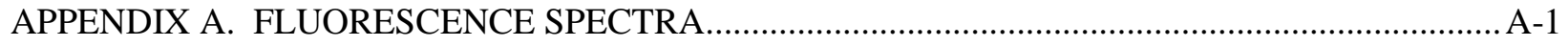

APPENDIX B. FUEL MARKER SENSOR SCHEMATICS AND LAYOUT ....................................... B-1

APPENDIX C. FUEL MARKER SENSOR MECHANICAL DRAWINGS ........................................ C-1

APPENDIX D. FUEL MARKER SENSOR BILL OF MATERIALS ................................................ D-1

APPENDIX E. FUEL MARKER SENSOR FIRMWARE SOURCECODE ......................................... E-1

APPENDIX F. FUEL MARKER SENSOR COMMAND SPECIFICATION.......................................

APPENDIX G. CUSTOM SIGHT GLASS DRAWINGS …................................................................ 



\section{LIST OF FIGURES}

A concept drawing of the proposed fuel tax evasion system ...... 1

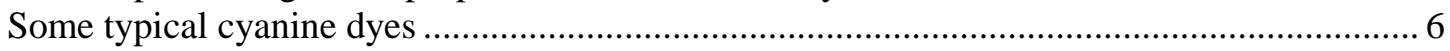

SiNaph, the chosen fuel-tax-evasion project marker, in its single macromolecule structure.

Some representative structures of several prominent fielded fuel dyes (A-C) and candidate dyes tested but not selected for the fuel tax evasion project (D and E) . Absorbance measurements with IR676 ("novel marker”) and agricultural diesel demonstrating lack of interference with fuel-tax-evasion project long wavelength marker's spectral region by Solvent Red 26/164-based agricultural diesel dye. Fluorescence spectra collected for pump diesel to determine whether competing fluorescence in the wavelength region of excitation above $630 \mathrm{~nm}$ would pose any significant interference, and none was noted

Fluorescence spectra collected for pump diesel containing the fielded fuel dye Solvent Yellow 124 to determine whether competing fluorescence in wavelength region of excitation above $630 \mathrm{~nm}$ would pose any significant interference, and none was noted ........ 14

Focused-range incremental calibration curve of IR676 in diesel on the prototype selfreferencing fuel marker sensor.....

Absorption spectra of IR676 at concentrations ranging from 0.052 ppm-9.4 ppm

collected after no wait time....

Emission spectra collected after no wait time of IR676 samples in diesel............................... 18

Emission spectra collected after 1 week of aging of IR676 samples in diesel...

Absorption spectra after 2 weeks of IR676 aging in diesel fuel........................................... 19

Emission spectra collected after 2 weeks of aging of IR676 samples in diesel ....................... 20

Change in emission spectrum of over time for the highest concentration of IR676 in diesel.

Various representations of an advanced macromolecule evaluated after stability failure of IR676 but ruled out due to price of $\$ 162.50$ for 1 milligram.............................................. 21 Naphthenic acids are prevalent in diesel and other heavier hydrocarbon fuels......................... 22 temperature (traces are overlapping) of SiNaph-marked diesel in the presence of tank

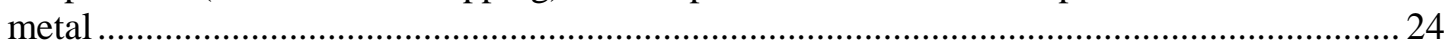

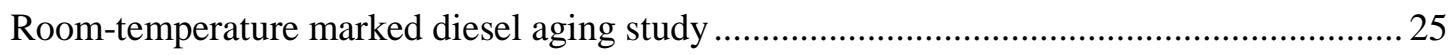

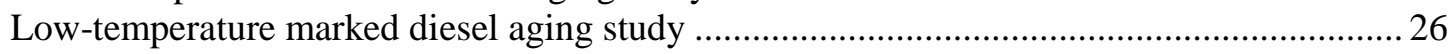

High-temperature marked diesel aging study .................................................................... 26

High-temperature unmarked diesel aging study ................................................................. 27

Blue trace (with peak at $770 \mathrm{~nm}$ ) shows initial absorbance response of "sister" dye in

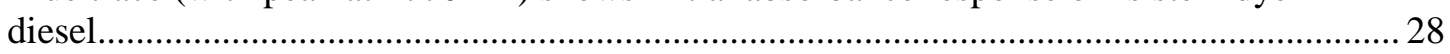

Degradation of sister dye at low, room, and high temperature in presence of tank metal....... 29

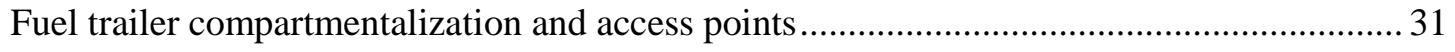

Sample GO switch installation to monitor valve position........................................................ 33

Setup for off-trailer evaluation of hatch and valve sensors .................................................... 34 


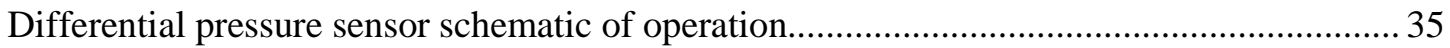

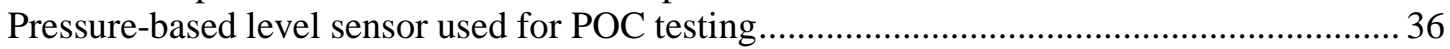

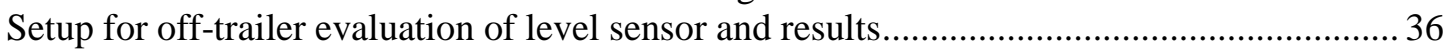

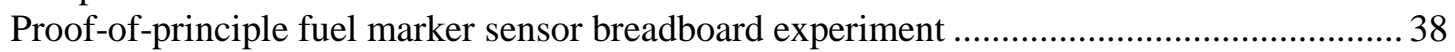

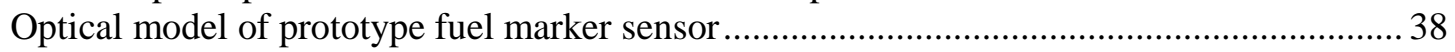

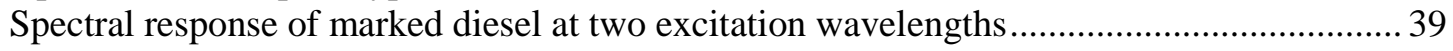

Focal point for blue LED (at $6 \mathrm{~mm}$ beyond cover window) ...................................................... 41

Focal point for red LED (located $6 \mathrm{~mm}$ beyond cover window).............................................. 41

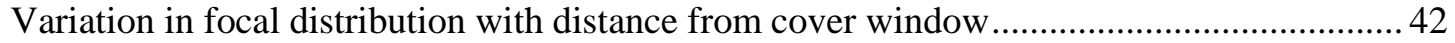

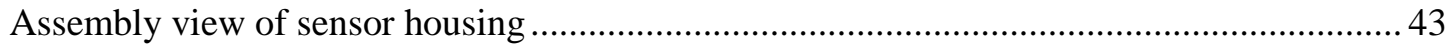

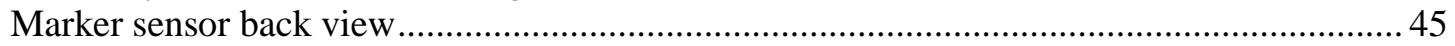

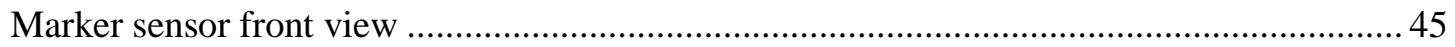

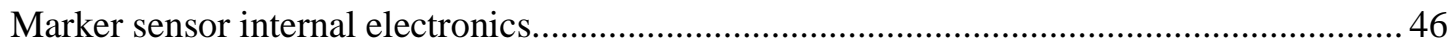

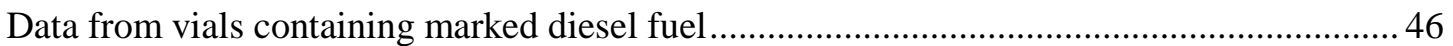

Schematic of sensor placement around fill valve on trailer compartment............................... 47

View of downpipe region on trailer showing sight glasses and bottom valves....................... 48

Sensors mounted on trailer fill valve and ORNL-designed sight glass ....................................4 49

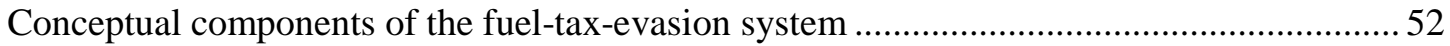

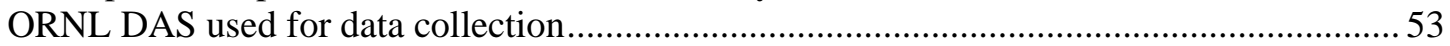

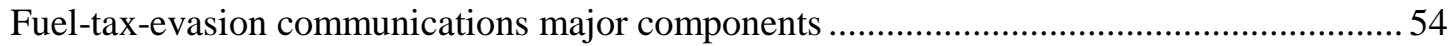

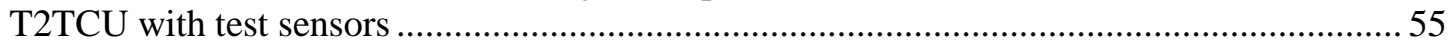

Fuel-Tax-Evasion Upload Data Service software ............................................................... 56

Fuel-Tax-Evasion Upload Data Service software_FTE settings ........................................... 57

Fuel Tax Evasion Upload Data Service software—started data retrieval service ....................5 57

Fuel-Tax-Evasion Upload Data Service software_communications software .........................5

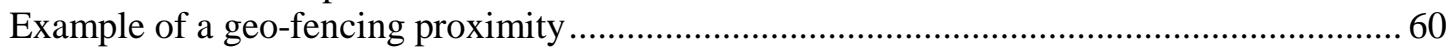

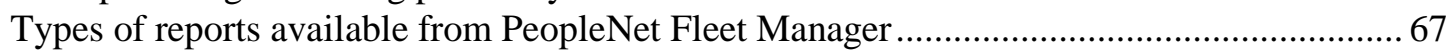

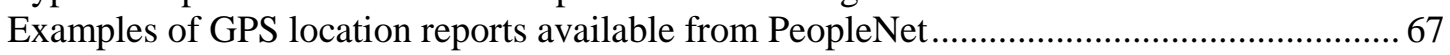

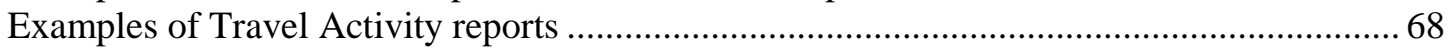

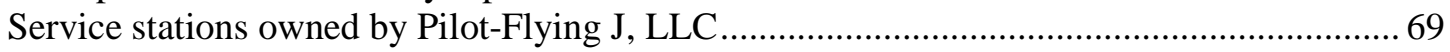

Pilot and Flying J service stations around the Knoxville, Tennessee, region.......................... 70

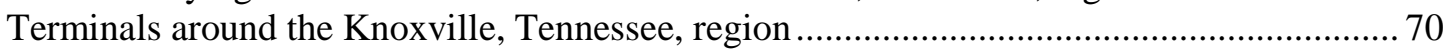

Examples of wholesalers and other customers served by Pilot tankers.................................... 71

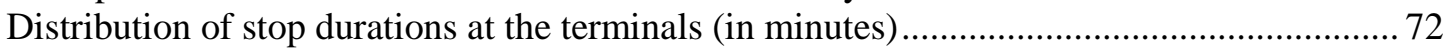

Distribution of stop durations at gas stations (in minutes) ....................................................... 73

Distribution of stop durations at other locations, including wholesale customers

(in minutes) ................................................................................................................... 73

67 Example of multiple route choices between a specific terminal and service station pair

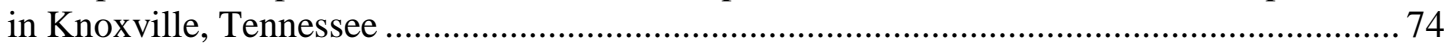

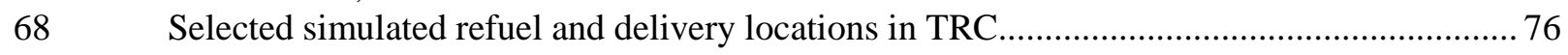

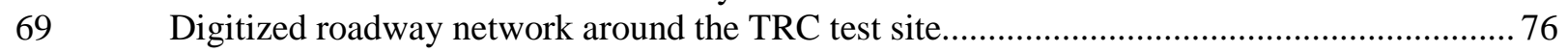

$70 \quad$ Predetermined nominal scenario route and geo-fencing boundary …................................. 78

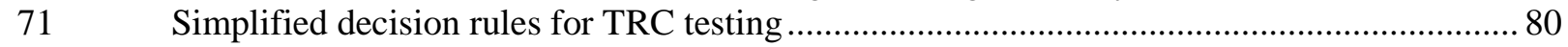

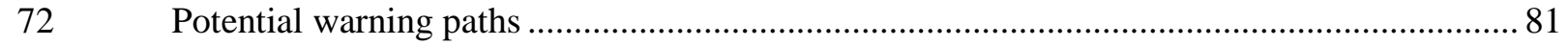

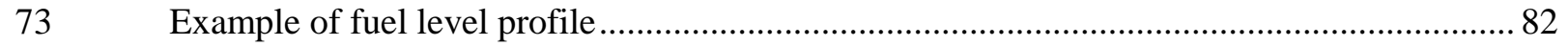

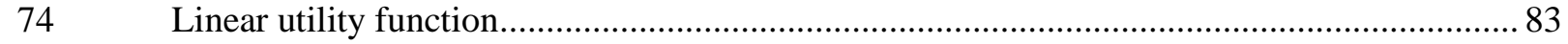

75 Example route for a simulation run in Knoxville, Tennessee .............................................. 84

76 A comparison of spatial location accuracy in data collected with the DAS device

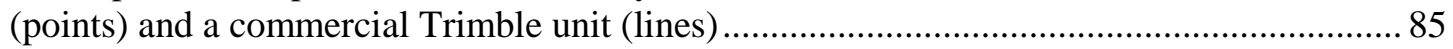

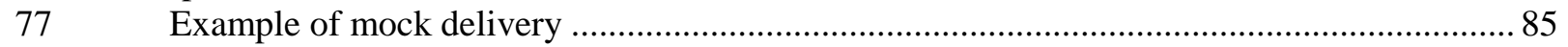

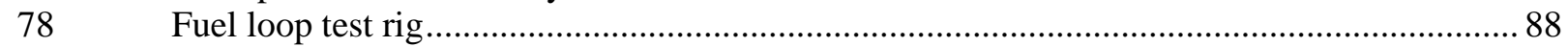




\begin{tabular}{|c|c|}
\hline 79 & Marker sensor data from fuel loop test at TRC.. \\
\hline 80 & Marker concentration data... \\
\hline 81 & DAS and T2TCU testing with fuel loop.... \\
\hline 82 & 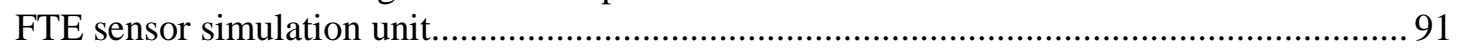 \\
\hline 83 & Travel routes based on GPS data from communications testing........... \\
\hline 84 & Simulated data collected during communications testing at TRC.... \\
\hline 85 & GPS plots of the pre-test routes at the TRC on March 22, $2011 \ldots \ldots \ldots . . . . .$. \\
\hline 86 & Sample scenario results from the pre-test at TRC on March 22, $2011 .$. \\
\hline 87 & 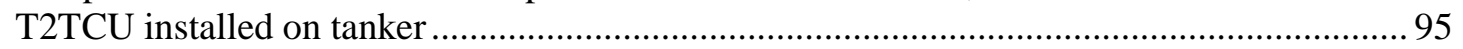 \\
\hline 88 & Marker sensor in situ operation verification..... \\
\hline 89 & Instrumented hatch located on top of tanker ....... \\
\hline 90 & Instrumented valve on tanker ........................... \\
\hline 91 & 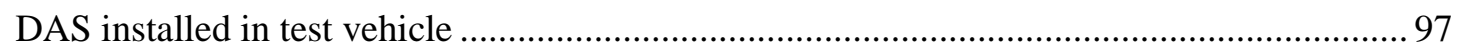 \\
\hline 92 & Test vehicle connected to TRC tanker for fuel transfer...................... \\
\hline 93 & Tanker routes at TRC during second day of field test...... \\
\hline 94 & Activity from offloading of compartment 1 under Scenario $1 \ldots .$. \\
\hline 95 & 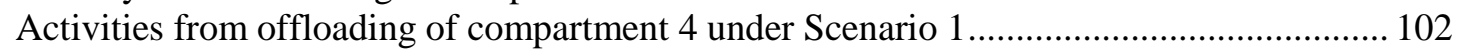 \\
\hline 96 & 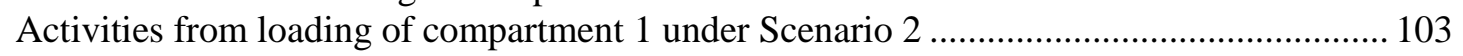 \\
\hline 97 & 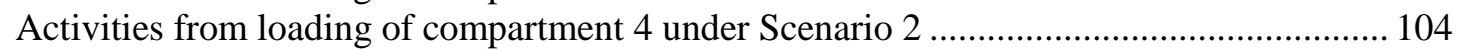 \\
\hline 98 & 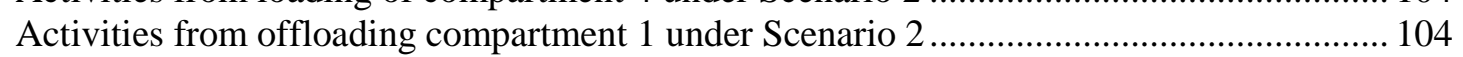 \\
\hline 99 & Activities from offloading compartment 4 under Scenario $2 \ldots$ \\
\hline 100 & 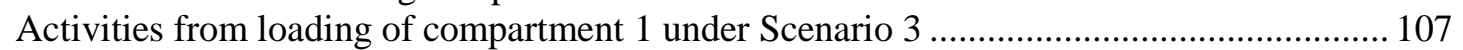 \\
\hline 101 & Offloading 50 gal at an unscheduled location under Scenario 3 ............... \\
\hline 102 & 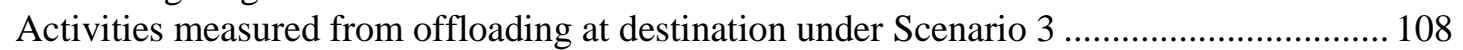 \\
\hline 103 & 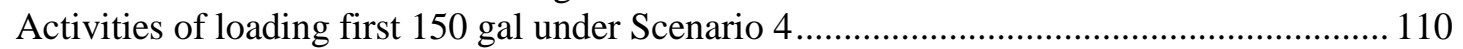 \\
\hline 104 & 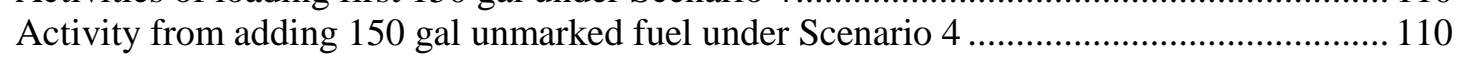 \\
\hline 105 & 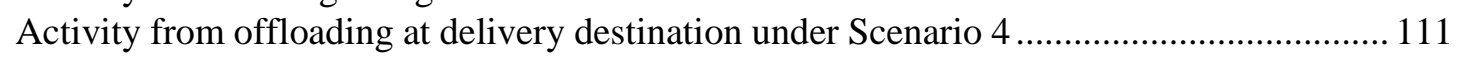 \\
\hline 106 & 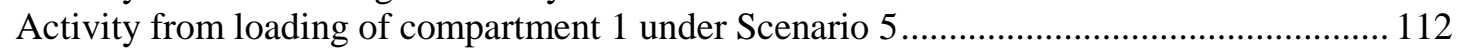 \\
\hline 107 & 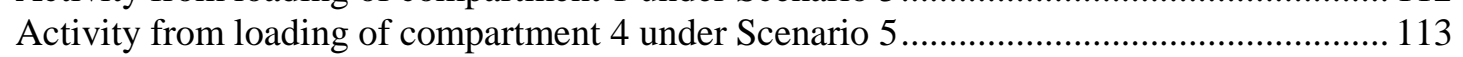 \\
\hline 108 & 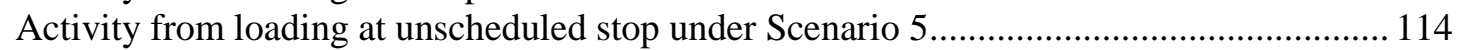 \\
\hline 109 & Activity from offloading of compartment 1 under Scenario $5 . . .$. \\
\hline 110 & 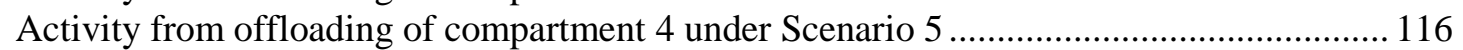 \\
\hline 111 & 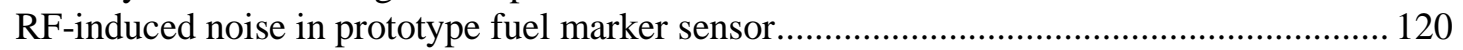 \\
\hline 112 & Data flow for recommended architecture .................. \\
\hline 113 & NovaChem capabilities for bulk manufacturing..... \\
\hline 114 & Valve with dust cover sensing apparatus................ \\
\hline 115 & Portion Fuel Level Profile at Stop t..... \\
\hline C.1 & Assembly of sensor components ......... \\
\hline C.2 & 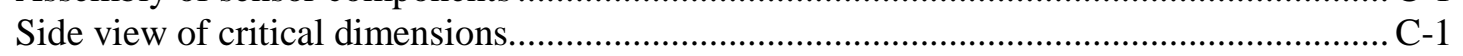 \\
\hline C.3 & Top view of critical dimensions .............. \\
\hline C. 4 & Critical holes and edges on PCB board layout.... \\
\hline C.5 & Critical PCB component placement (front)........... \\
\hline C.6 & Critical PCB component placement (back) ....... \\
\hline
\end{tabular}





\section{LIST OF TABLES}

Table

Page

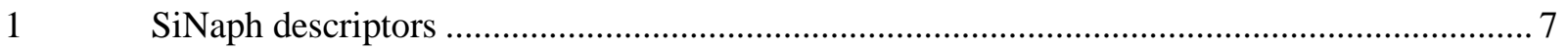

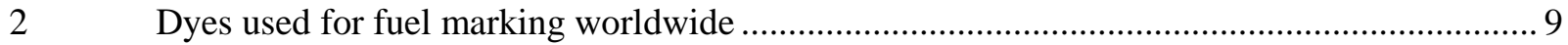

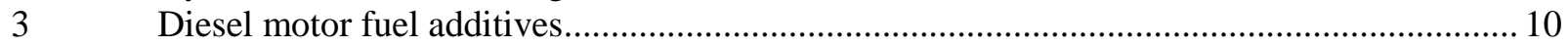

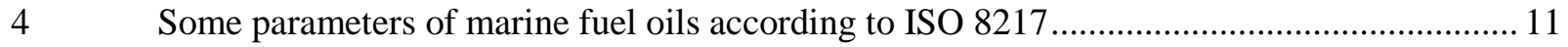

$5 \quad$ Summary of sensors per fuel tanker compartment ............................................................ 56

$6 \quad$ Summary of FTE-related costs for the proposed architecture ............................................. 126 



\section{ACRONYMNS AND ABBREVIATIONS}

$\begin{array}{ll}\text { BOS } & \text { Back Office System } \\ \text { CCM } & \text { Chassis Communications Module } \\ \text { COTS } & \text { Commercial off-the shelf } \\ \text { CSV } & \text { Comma Separated Value } \\ \text { DAS } & \text { Data Acquisition System } \\ \text { DOE } & \text { United States Department of Energy } \\ \text { DOT } & \text { United States Department of Transportation } \\ \text { D-S } & \text { Dempster Shafter } \\ \text { FHWA } & \text { Federal Highway Administration } \\ \text { FSK } & \text { Frequency Shift Key } \\ \text { FTE } & \text { Fuel Tax Evasion } \\ \text { GIS } & \text { Geographical Information System } \\ \text { gpm } & \text { Gallons per minute } \\ \text { GPS } & \text { Global Positioning System } \\ \text { IC } & \text { Integrated Circuit } \\ \text { IDS } & \text { Intelligent Decision System } \\ \text { LED } & \text { Light Emitting Diode } \\ \text { ml } & \text { milliliter } \\ \text { mm } & \text { millimeter } \\ \text { mW } & \text { milliwatt } \\ \text { NIR } & \text { Near Infrared } \\ \text { nm } & \text { nanometer } \\ \text { ORNL } & \text { Oak Ridge National Laboratory } \\ \text { PCB } & \text { Printed Circuit Board } \\ \text { Pilot } & \text { Pilot Travel Centers, LLC (including Flying J Travel Centers) } \\ \text { POC } & \text { Proof of Concept } \\ \text { ppb } & \text { Parts per billion } \\ \text { ppm } & \text { Parts per million } \\ \text { psi } & \text { Pounds per square inch (gauge) } \\ \text { RF } & \text { Radio frequency } \\ \text { SE VDA } & \text { Southeastern Vehicle Dynamic Area } \\ \text { SiNAPH } & \text { 2,3-naphthalocyaninato-bis(trihexylsiloxy)silane, bis(trihexylsiloxy)silicon 2,3- } \\ \text { T2TCU } & \text { Tractor to Trailer Communications Unit } \\ \text { TRC } & \text { Transportation Research Center } \\ \text { UV } & \text { Ultraviolet } \\ \text { VDC } & \text { Voltage Direct Current } \\ \text { WRC } & \text { Winding Road Course } \\ \text { WRI } & \text { Wireless Roadside Inspection } \\ & \end{array}$





\section{ACKNOWLEDGMENTS}

ORNL would like to thank Pilot Travel Centers, LLC, for their gratis partnership in this proof-of-concept testing. Pilot generously donated the use of their vehicle and fuel for the testing. In addition, Doug Drummer, David Breeding, driver Nick Peaker, and others provided the research team with valuable information regarding fleet operations and access to fleet data; they also allowed the research team to observe operations firsthand in order to better design the test systems, formulate test plans, and select instrumentation.

ORNL would also like to thank the staff at the Transportation Research Center for their testing support. Seth Reynolds, Ken Webster, and drivers, technicians, and other support staff made it possible to complete the full set of tests, adapting to various weather and technical issues which arose during the testing. 



\section{EXECUTIVE SUMMARY}

The goal of this research was to provide a proof-of-concept (POC) system for preventing non-taxable (non-highway diesel use) or low-taxable (jet fuel) petrochemical products from being "blended" with taxable fuel products and preventing taxable fuel products from cross-jurisdiction evasion. The research worked to fill the need to validate the legitimacy of individual loads, offloads, and movements by integrating and validating, on a near-real-time basis, information from global positioning system (GPS), valve sensors, level sensors, and fuel-marker sensors.

There were five innovative aspects to this pilot research:

1. Instead of relying on a single solution, fuel tax evasion (FTE) will be prevented through the integration of multiple measures.

2. This research recognized that there is not a "silver bullet" for preventing FTE. As such, solutions will be tailored to address specific scam(s).

3. Inline sensors were developed and evaluated for their feasibility to authenticate the presence and concentration of fuel markers in the fuel.

4. This research integrated advances in sensor technology, wireless communications, vehicle tracking, and information analysis. An integrated sensor suite, communications system, and data acquisition platform were developed. The platform allowed the use of various sensor modules and communication methods to fit specific applications. Local data storage was also provided for backup purposes.

5. The simulated control center was designed to be able to communicate with law enforcement personnel in a timely manner to allow them to investigate potential fuel diversion activities.

The inclusion of a chemical marker and sensor system as an indicator of fuel dilution was intended to serve as a confirmation of illegal activities associated with evasion of fuel tax. The ORNL team successfully identified and rigorously tested a fuel marker with the following characteristics: compatibility with fuels and engines, production of no objectionable emissions or by-products, no visibility to the naked eye, chemical stability under thermal extremes over the period of months, and high enough optical yields to produce detectable fluorescence in the parts-per-billion range. The optical and aging chemistries of candidates other than the marker chosen were investigated along the way, as was the performance of less expensive off-the-shelf alternative markers. The SiNaph system was chosen because it proved to be the purest and therefore most stable over the long term and the most optically reliable of those studied. Ultimately, this marker can be reliable as a long-term fuel marker and for dilution sensing. A suite of sensors attached to a fuel transport vehicle provided the critical information needed to evaluate whether or not a FTE has occurred. In the most general terms, the sensor suite must furnish information capable of determining whether any of the access points into the trailer were opened, how much fuel is present (fuel level), and whether the fuel in the truck is the fuel on which taxes were paid at the load point. The marker sensor was developed in-house by ORNL, and mounting solutions were designed inhouse with commercial, off-the-shelf sensors for the pressure sensors and the valve and hatch position sensors. In contrast to the hatch, valve, and level sensors, the marker sensor required that a selfreferencing sensor and sensing technique be developed that had no commercial counterpart.

The communications system required to accomplish the needs of the FTE effort was able to collect and format sensor signals from the trailer (switches, level sensor, marker sensor, weight), convey the sensor signals from the trailer to the tractor without the addition of any cabling or wiring between the tractor and trailer via power line communications, receive sensor data from the trailer and condition and format the sensor signals for acceptance by the onboard computer, and be capable of sending the data packets to a back office system (BOS) for processing. Due to the restrictions imposed by the use of the RS485 protocol for the fuel marker sensor, a suitable commercial off-the-shelf solution was not identified for the 
tractor-to-trailer communications unit (T2TCU), so the development was contracted to private industry. The data posted by the T2TCU was read by the ORNL data acquisition system (DAS) at $1 \mathrm{~Hz}$. A realtime communications link was established between an ORNL computer and the DAS, was made possible by the onboard cellular modem. This streaming data was monitored and split into short files capturing 5min increments of data; these files were made available to the evidential reasoning component for analysis via automated uploads to a server.

As part of this research study, the development of a POC intelligent Geographical Information System (GIS)-based system was called for to facilitate near-real time notification of suspicious variation in standard delivery processes, tracking the tanker as it travels from the origin (e.g., terminal) to its destinations (e.g., retail outlet) on the delivery route. The control center, where the "system" resides, monitors sensor activities occurring at and between the terminal loading of the tank truck and the unloading at the retail outlet. By applying sensor technologies in combination with tools developed based on data fusion and validation techniques, suspicious activities could be identified in real time. The intelligent GIS-based system developed under this research study performed two major functions: geofencing and evidential reasoning. Data related to fuel delivery operations were obtained from several sources including observations made during "shadowing” of tanker drivers during normal operations, notes gathered from interviews with Pilot managers, and analysis of specific PeopleNet reports. Supplemental information was also collected through web searches and internal communications with the Sensor Team. In addition, simulations were conducted to generate sensor information using a passenger car with connections to the DAS.

The technical approach taken in this activity provides a proactive rather than a reactive capability to determine if non-taxed fuel is being diverted for use in motor vehicles. An integrated sensor, communications, and power management platform will be installed on the tank truck. The sensor units were able to identify, in real time, the opened or closed status of the tank truck valve, the level of fuel that is being loaded or unloaded, and the amount of fuel marker in the fuel. The tanker also had a GPS unit for tracking. This was tied to a geo-fencing program that identified stops along the vehicle's route. The geofencing program was integrated with the evidential reasoning system to provide, when combined with the transportation manifest, an appropriate level of warning of suspicious activities.

After a thorough search for commercially available materials to build each system component to detect FTE, ORNL laid out a test methodology whereby each system component was tested individually prior to the assembly of the complete system. The fuel marker components were first designed on a benchtop system within a lab where each sensor component of the fuel marker sensor suite was tested. Once the benchtop system was proven, the assembly was scaled up for retrofit to a fuel tanker. Due to liability issues, ORNL contracted with the Transportation Research Center (TRC) in East Liberty, Ohio, to conduct the field test on a closed track. ORNL began by testing the fuel loop assembly on the tanker at TRC to see how the assembly processed full-scale fuel flow and to rule out fuel leaks. That test was successful and was followed by a field test within an instrumented tanker using FTE scenarios.

The POC testing does not lend itself to real-world data collection due to the non-production nature of the equipment and sensors used and the need to control the testing steps and maneuvers to gather the required data. These factors and the general concern for staff safety and the safety of the Pilot Travel Centers, LLC (Pilot) vehicle led to a decision early in the project to conduct this POC vehicle-based data collection at a closed test facility. The TRC in East Liberty, Ohio, was chosen based on the size of its facility (several miles of roadway), experience with commercial vehicle testing, and ability to handle the needed fuel transfers.

Prior to the field test with the tanker, preliminary testing was conducted at TRC to test out the marker sensor hardware and software under conditions similar to those which would be experienced during the 
field test; this opportunity was also taken to test the prototype T2TCU with a functioning marker sensor. Additionally, the simulated sensor data was collected as the test scenarios were performed on-site at TRC to confirm the DAS-to-BOS communications and collect data to fine-tune the evidential reasoning system.

The Field Test conducted at TRC using the Pilot's tanker tractor-trailer involved the full ORNL end-toend FTE system. The test vehicle was moved from location to location within the TRC reservation to simulate loading and offloading locations such as a fuel terminal and retail fuel sales location and included several test scenarios which mimicked real-world operations.

This POC testing was successful, proving the feasibility of this FTE detection solution. In addition, this research provided valuable information regarding where future research efforts are needed to further develop such a system. 



\section{INTRODUCTION}

\subsection{BACKGROUND}

Revenues from motor fuel and other highway use taxes provide the primary source of funding for the United States' transportation system, and ensuring all of these taxes are collected, remitted, and credited to the Highway Trust Fund is a priority for the U.S. Department of Transportation (DOT) Federal Highway Administration (FHWA). In the past, loss of revenue due to tax evasion has been estimated to range from $\$ 1$ billion annually to as much as $25 \%$ of total revenues. After the point of taxation was changed for both gasoline (1988) and diesel fuel (1994), significant increases in tax revenue were realized which were assumed to be due to decreased evasion. Additionally, diesel fuel that was to be used for offroad (non-taxable use) purposes was marked with a red dye, making it easier for on-road enforcement personnel to identify the propulsion fuel as having had all proper taxes paid prior to its use in the vehicle. However, there are still a number of extensive and convoluted schemes to evade fuel taxes that cannot be addressed by a single solution. Rather, a supply-chain-based solution is needed. This research used evidential reasoning techniques, fuel markers, sensor devices, and vehicle tracking devices to monitor, track, and detect the transfer and movements of petroleum products between different locations and determine the "legitimacy" of the movements and fuel loading/unloading (Fig. 1). This fuel tax evasion (FTE) detection system has the potential to reduce or eliminate a number of FTE schemes, resulting in millions of dollars of additional revenue in the Highway Trust Fund.

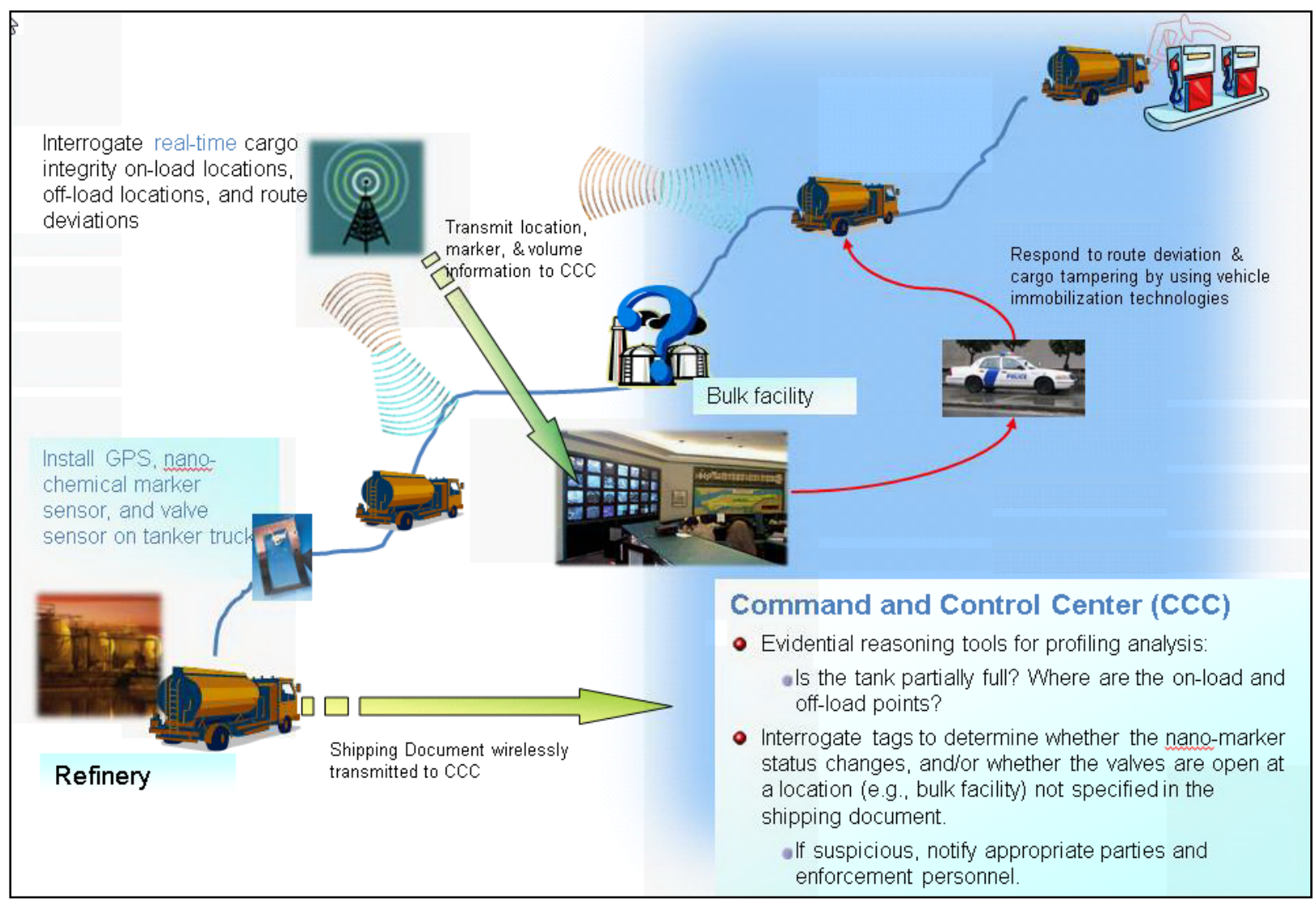

Fig. 1. A concept drawing of the proposed fuel tax evasion system. 
For Federal tax purposes, the point of taxation for gasoline and diesel fuel is at the point of its removal from bulk storage at the terminal rack. Approximately half of the states in the United States have the same point of taxation. However, the rest of the states have a point of taxation at the wholesaler/distributor level or below. This presents additional challenges in tracking untaxed fuel after it leaves the bulk storage. Some of the technologies introduced in this research project would be useful in tracking the "below the rack" (after the product leaves the bulk terminal) movement. The terminal rack is the facility where fuel from bulk storage tanks is loaded into tanker trucks for delivery to retail stations or bulk users. Only dyed diesel fuel may be removed from bulk storage without payment of the Federal diesel fuel excise tax, and the taxes in various states that have similar dyed diesel statutes. Dyed diesel is used by farmers, fishermen, and others for off-road proposes. There are no dyeing requirements for gasoline.

The current approaches in identifying and preventing FTE are primarily through fuel tax enforcement, electronic registration and data reporting, and processing and analyzing fuel tax data. The enforcement activities include checking highway diesel users for dyed fuel in their tanks and audits of the gasoline tank truck delivery system. These approaches are time consuming, and they attempt to discover and investigate evasions after they have taken place. This research proposes to monitor and determine, in near real time, the "legitimacy" of every loading, offloading, and movement of petroleum products. By so doing, this proposed solution provides enforcement staff with intelligence on a potential diversion of fuel or other tax evasion during such an event.

\subsection{GOALS AND OBJECTIVES}

The goal of this research was to provide a proof of concept (POC) system for preventing (1) non-taxable (non-highway diesel use) or low-taxable (jet fuel) petrochemical products from being "blended" with taxable fuel products and (2) taxable fuel products from cross-jurisdiction evasion. The research worked to fill the need to validate the legitimacy of individual loads, offloads, and movements by integrating and validating, on a near-real-time basis, information from global positioning system (GPS), valve sensors, level sensors, and fuel-marker sensors.

There were five innovative aspects to this pilot research.

1. Instead of relying on a single solution, FTE will be prevented through the integration of multiple measures.

2. This research recognized that there is not a "silver bullet" for preventing FTE. As such, solutions will be tailored to address specific scam(s).

3. Inline sensors were developed and evaluated for their feasibility to authenticate the presence and concentration of fuel markers in the fuel.

4. This research integrated advances in sensor technology, wireless communications, vehicle tracking, and information analysis. An integrated sensor suite, communications system, and data acquisition platform were developed. The platform allowed the use of various sensor modules and communication methods to fit specific applications. Local data storage was also provided for backup purposes.

5. The simulated control center was designed to be able to communicate with law enforcement personnel in a timely manner to allow them to investigate potential fuel diversion activities.

The major benefit to the highway programs is the potential to increase the collection of fuel tax revenue. It is estimated that Highway Trust Fund could be depleted in the near future. This research will substantially increase the resources needed to build and maintain the transportation infrastructure.

The technical approach taken in this activity provides a proactive rather than a reactive capability to determine if non-taxed fuel is being diverted for use in motor vehicles. An integrated sensor, communications, and power management platform were installed on the tank truck. The sensor units were 
able to identify, in real time, the open or closed status of the tank truck valve, the level of fuel that is being loaded or unloaded, and the amount of fuel marker in the fuel. The tanker also had a Global Positioning System (GPS) unit for tracking. This was tied to a geo-fencing program that identified stops along the vehicle's route. The geo-fencing program was integrated with the evidential reasoning system to provide, when combined with the transportation manifest, an appropriate level of warning for suspicious activities.

This system allowed for near-real-time notification of any suspicious variation in standard delivery processes as the tanker moved from the origin (e.g., terminal) to the destinations (e.g., retail outlet) on its delivery route. Therefore, the key metrics to measure the impacts of this research include, but are not limited to,

1. the propensity and accuracy of detecting illegal blending and bootlegging;

2. the ease and the costs (including both direct and indirect costs) of deploying the proposed system;

3. the frequency and speed by which the communications center is notified of a potential illegal activity; and

4. the transparency of the detection system to the carriers' operations.

A testing procedure was developed to measure these key metrics and data collection, and analysis plans were developed to quantify the impacts of the research.

\subsection{ROLES AND PARTNERSHIPS}

Oak Ridge National Laboratory (ORNL) provided the technical and managerial support for the project. This role included the development and integration of sensors, tracking devices, fuel markers, communications software, evidential reasoning software, geographical information system (GIS) software, and display interfaces.

Pilot Travel Center, LLC (Pilot), which owns carrier and retail outlets, donated diesel fuel to conduct the tests scenarios that were conducted in the field operational test. Tracking devices, valve position indicators, fuel-marker sensors, power supply, and a data acquisition system (DAS) fitted with wireless communication capability were installed on a tanker truck from Pilot's carrier fleet at the Transportation Research Center (TRC) in East Liberty, Ohio.

ORNL contracted with Air-Weigh to build a specialized trailer-to-tractor communications unit (T2TCU) to interface with the sensor suite that was mounted on the tanker. This device transferred data from the sensors through the trailer's data bus to the truck's in-cab DAS. More detailed discussions regarding these devices follow in Sects. 3 and 4 of this report.

\subsection{OVERVIEW OF TEST METHODOLOGY}

After a thorough search for commercially available materials to build each system component to detect FTE, ORNL laid out a test methodology whereby each system component was tested individually prior to the assembly of the complete system. The fuel marker components were first designed on a benchtop system within a lab where each sensor component of the fuel marker sensor suite was tested. Once the benchtop system was proven, the assembly was scaled up for retrofit to a fuel tanker. Due to liability issues, ORNL contracted with TRC in East Liberty, Ohio, to conduct the field test on a closed track. ORNL began by testing the fuel loop assembly on the tanker at TRC to see how the assembly processed full-scale fuel flow and to rule out fuel leaks. That test was successful and was followed by a field test within an instrumented tanker using FTE scenarios. 



\section{FUEL MARKER}

The inclusion of a chemical marker and sensor system as an indicator of fuel dilution was intended to serve as a confirmation of illegal FTE activities within the larger, multifaceted approach to mitigating revenue loss from illicit transfers of fuel. The ORNL team has successfully identified and rigorously tested a fuel marker that has the following characteristics:

- compatibility with fuels and engines,

- production of no objectionable emissions or by-products,

- no visibility to the naked eye,

- chemical stability under thermal extremes over the period of months, and

- optical yields high enough to produce detectable fluorescence in the parts-per-billion range.

The optical and aging chemistries of candidates other than the marker chosen were investigated along the way, as was the performance of less-expensive off-the-shelf alternative markers. However, the SiNaph system was chosen because it proved to be the purest and therefore the most stable over the long term and the most optically reliable of those studied. Ultimately, this marker can be reliable as a long-term fuel marker and for dilution sensing. In going forward, a less expensive purchase route for the same highquality marker could be followed given lead time of 6 months. Most integral to a real-world implementation of the fuel marker system would be the development of a specialty manufacture niche that could produce a SiNaph marker for the lowest possible cost at a lead time of 1 to 2 years.

\subsection{FUEL MARKER BACKGROUND}

The Fuel Marker Team sought to implement their knowledge of fluorescence and tagging chemistries within the framework of automotive and diesel fuel applications to the benefit of the multi-strategy POC research approach for mitigating FTE. This section will introduce the fundamental principles of fluorescence; give a working history of fuel tagging via optical markers; and detail all the work that led to the final selection of a marker. Other project-critical topics such as the density of diesel, the integral nature of purified optical material, and the effect of mixing very highly concentrated, nearly saturated solutions into pure diluent will be covered as well.

\subsubsection{Fluorescence}

Molecular fluorescence results when light, commonly in the ultraviolet, visible, or infrared region of the electromagnetic spectrum, impinges upon a compound and promotes the molecule or system of molecules and atoms to an excited (increased internal energy) electronic state. As the molecule or system of molecules and atoms relaxes to the more statistically favored, lower-energy ground state, some of the released energy can be detected as emitted light. The energy value of this emitted light reflects aspects of the chemical structure of the fluorescent compound, and a graph of emission intensity versus energy value (wavelength) can create a descriptor unique to a molecule or, more accurately, a family of molecules. Because certain families of molecules can be excited and made to emit fluorescence in wavelength regions where most other ambient compounds do not, the emitted light of a fluorescent marker offers a strong, high signal relative to lower background levels. When these properties are exploited as a tagging strategy in order to monitor the increase, decrease, or migration of a fluorescent species, extremely sensitive detection is possible, even in optically dense or "murky" solutions. Some examples of the double bonds within an organic molecule that offer "loosely held" electrons that can be excited by wavelengths of visible energy are shown in the generic and widely available cyanine pure organic dyes in Fig. 2. A series of such pi bonds, the extended association of multiple double bonds within a molecule or complex, can be referred to as conjugation. 


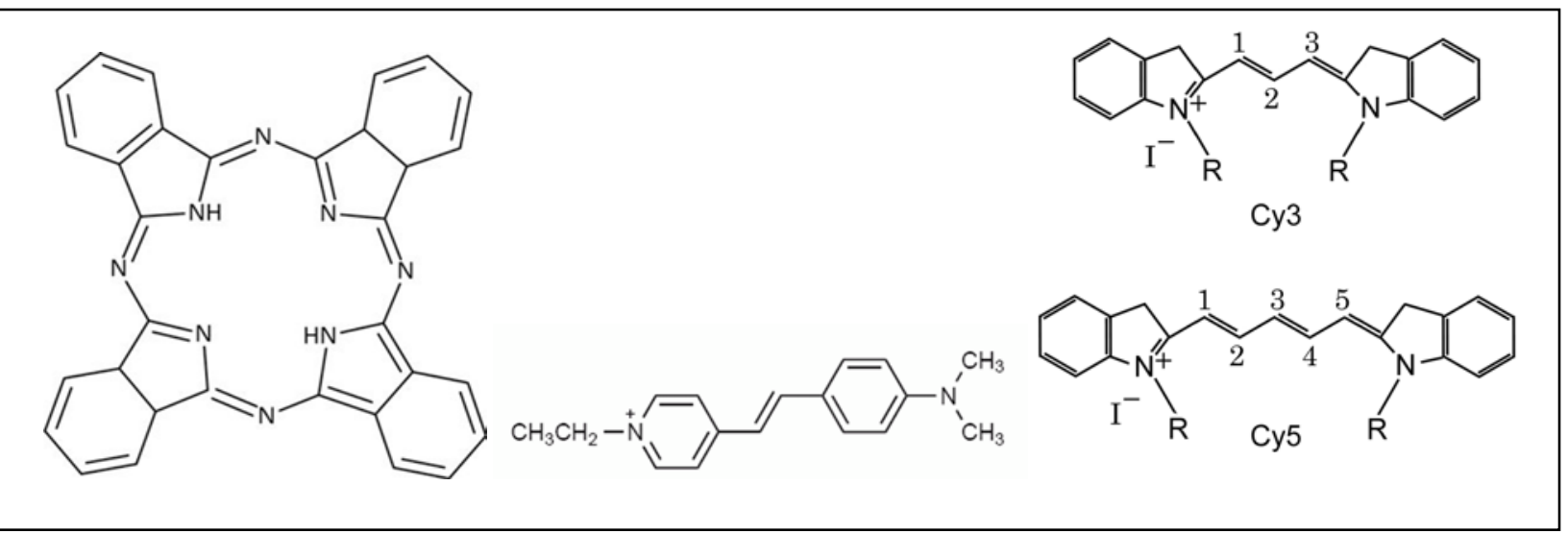

Fig. 2. Some typical cyanine dyes. Note the conjugation, or extended double bond—single bond-double bond pattern, within the molecules that leads to fluorescence. (Image source: http://en.wikipedia.org/wiki/Cyanine)

The final FTE marker that was chosen is 2,3-naphthalocyaninato-bis(trihexylsiloxy)silane, bis(trihexylsiloxy)silicon 2,3-naphthalocyanine, (henceforth denoted as "SiNaph"). SiNaph's structure is shown in Fig. 3.

Table 1 includes basic physical and spectroscopic properties, as well as useful identifying numbers, to facilitate further searches regarding this compound. It is a complicated synthetic product from cyanine roots.

For the purposes of the FTE project, the fluorescent marker system was ultimately based on this fluorophore, whose emission results from highly extended conjugation and whose stability was facilitated by organic ligand complexation around a silicon core ion. These "flexible" organo-silicon chemical linkages offer dual advantages to the SiNaph molecule. The central ion allows long-range electronic conjugation for high fluorescent yield and thermal stabilization. This structural robustness of the macromolecule cushions the blow of acidic diesel components and thermal effects, thereby preventing molecular degradation and its attendant decrease in signal due to anything other than concentration dilution. Other, competing reductions in fluorescent signal emitted from the chosen marker molecules include degradation of the marker by chemical attack from diesel components or additives to diesel (naphthenic acids, peptizing agents, surfactants) as well as chemical degradation due to absorbance of excess molecular-level heat, the other relaxation route of an optically excited molecule.

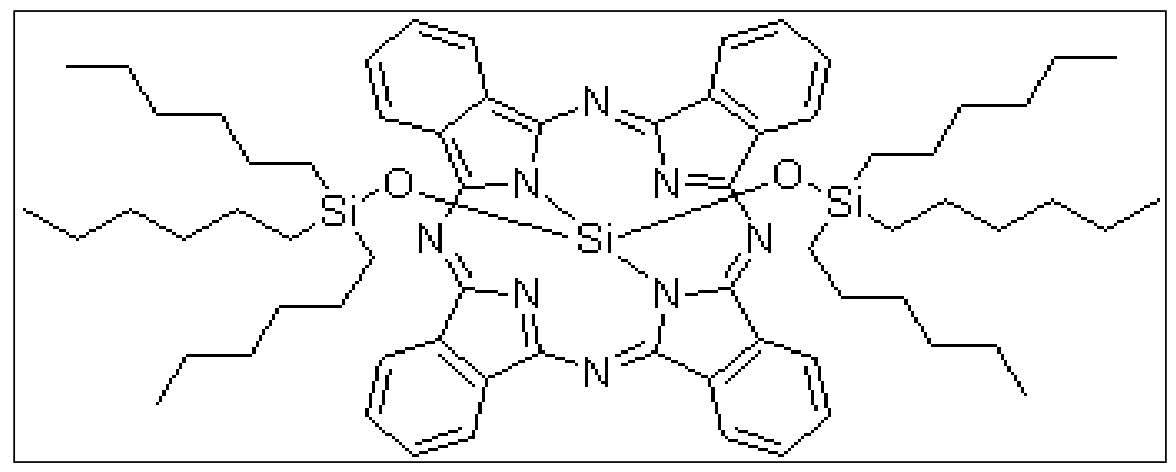

Fig. 3. SiNaph, the chosen fuel-tax-evasion project marker, in its single macromolecule structure. 
Table 1. SiNaph descriptors

\begin{tabular}{|c|c|}
\hline Name: & Silicon 2,3-naphthalocyanine bis(trihexylsilyloxide) \\
\hline Synonym: & $\begin{array}{l}\text { 2,3-Naphthalocyaninato-bis(trihexylsiloxy)silane, } \\
\text { Bis(trihexylsiloxy)silicon 2,3-naphthalocyanine }\end{array}$ \\
\hline CAS number: & $92396-88-8$ \\
\hline Beilstein registry number: & 4348502 \\
\hline Linear formula: & $\mathrm{C}_{84} \mathrm{H}_{102} \mathrm{~N}_{8} \mathrm{O}_{2} \mathrm{Si}_{3}$ \\
\hline Molecular weight: & 1340.02 \\
\hline Melting point: & $270^{\circ} \mathrm{C}$ (dec.)(lit.) \\
\hline \multirow[t]{2}{*}{ UV absorption: } & $\lambda_{\max } 729$ nanometer $(\mathrm{nm})$ \\
\hline & $\lambda_{\max } 774 \mathrm{~nm}(2 \mathrm{nd})$ \\
\hline
\end{tabular}

\subsubsection{Marking or Tagging of Fuels}

Marking, tagging, or dyeing of heating and vehicular fuels has been implemented widely over the last three decades to differentiate lower taxed and/or higher polluting fuels used for industrial endeavors, aviation, marine navigation, and home heating from automotive fuels. The greater volume of these onroad fuels used results in a significant revenue stream from increased tax rates. In addition, environmental regulatory agencies provide guidelines regarding on-road fuels as a source of sulfur, nitrogen oxides, and groundwater contamination. Significant pollution could occur were there not strict limitations imposed to keep the levels of insulting species as low as possible for on-road fuels. Most currently fielded strategies to tag fuel mark the lower-taxed stream and require sampling of the fuel from the consumer end. The FTE project has proved an approach to mitigating FTE at the distributor end for on-road automotive diesel via a more-real-time, lower-concentration, less-perceptible fuel marking strategy that relies upon fluorescence, not absorbance or titration. The discussion below introduces the two main marker systems already fielded, one in Europe and one domestically, to create a context for the novel nature of the FTE project proved in concept by the ORNL team.

Solvent Yellow 124 is a yellow “azo dye” (organic chromophores containing amino acid groups that can be neutral or ionic) added to fuels not intended for automotive use in the European Union countries to differentiate those road vehicle fuels from home heating fuels. It is a marker used since August 2002 to distinguish diesel fuel intended for heating from a higher-taxed motor diesel fuel. The dye is added to lower-taxed non-road fuels in amounts of $6 \mathrm{mg} / \mathrm{L}$ or $7 \mathrm{mg} / \mathrm{kg}$ (or ppm) under the name Euromarker. This dye can be easily hydrolyzed with acids; splits off the acetal group are responsible for its miscibility in hydrocarbons and lead to the formation of a hydrophilic form similar to the common methyl orange food and clothing dye. A pH value in the acidic range leads to a red colorimetric reading detectable in fuel at 300 parts per billion via extraction to hydrochloric acid. These operations require manpower, sample destruction, and time to identify the marker in fuel samples, steps that the FTE project and the ORNL team sought to avoid incorporating in the ultimate deployment of the tax evasion mitigation strategy. The amount of Solvent Yellow 124 added to the fuel is determined via a much more tedious method than our online sensor, namely by removing samples from the fuel lines and by measuring the Euromarker content in the fuel. In this way, it is possible for customs agents (equivalent to our tax officers) to calculate how much of the low-taxed fuel was added to the legal one, regardless of the usage of such scam techniques as duel fuel tanks or dummy reservoirs. Current trends in the European Union countries include efforts by Great Britain to circumvent the "laundering” or chemically bleaching/leaching of dye out of the diluted fuel as well as replacement for a biological marker in Denmark. 
Solvent Red 26, also known as C.I. 26120, is also an azo dye created synthetically and utilized for colorimetric distinction of higher-sulfur-content, tax-reduced, or tax-exempt agricultural and heavy equipment diesel and lighter home heating oils from automotive diesel fuel. Colorimetric markers are measured by the absorbance of light, not their emitted light detection as in the case with fluorimetric markers such as SiNaph. Solvent Red 26, whose chemical formula is $\mathrm{C}_{25} \mathrm{H}_{22} \mathrm{~N}_{4} \mathrm{O}$, or 1-[[2,5-dimethyl-4[(2-methylphenyl)azo]-phenyl]azo]-2-naphthol, is soluble in oils and insoluble in water. Replacement of Solvent Red 26 with Solvent Red 164 is increasingly common due to its longer alkyl chains which facilitate an increased solubility in the hydrocarbon matrices it marks. The IRS, which governs taxation, requires $11.13 \mathrm{mg} / \mathrm{l}$ Solvent Red per liter of fuel; the EPA, which governs sulfur regulations, requires fivefold less. Both values are based on the integrated optical spectroscopic response of the colorimetric dye in a given wavelength range, since the batch-to-batch variations in the chemistry of unrefined dyes such as these can produce significant variations in the exact absorbance or fluorescent (in the case of unrefined SiNaph lots) responses and magnitudes.

Table 2 presents a wider swath of the fuel dyes used worldwide. All of these are either sampled (not real time/online), colorimetric, titration, or placed in off-road fuels.

Shortcomings of currently fielded fuel marking methodology that our approach seeks to mitigate include lack of response sensitivity in colorimetric systems, lack of a real-time measurement capability, and personnel-intensive monitoring scheme.

\subsection{SELECTION OF FUEL MARKER}

Two aspects of the fuel marker figured equally importantly in the selection of a compound to indicate illegal fuel dilution. Generally these descriptors fall into the categories of optical performance and automotive compatibility.

The first is the requirement attendant to the optical and chemical characteristics of a dye. Along this line, the compound needed to

- $\quad$ exhibit intense (high yield) fluorescence to minimize the amount of marker needed, thereby minimizing cost and ensuring the concentration of marker in the fuel falls below the EPA rule of $3 \%$ difference for fuel additives;

- emit fluorescence in a spectral region distinct from that of diesel and other fielded fuel colorants for clear identification of the concentration level; and

- be highly miscible with diesel fuel to ensure the concentration of marker is consistent throughout.

The second requirement centers on the fact that this marker system will exist in a combustible fuel system. Hence, in addition to the optical requirements detailed above, the compound must also

- pose no calculable threat to an internal combustion engine via accumulations of salts or heavy metals within fuel systems, and

- any non-combustible component of the dye would need to be present at levels low enough to be comparable to other inorganic contributions to fuel systems due to existing chemicals present such as lube oil.

Identifying a fuel marker that met these requirements was an iterative process, beginning with surveys of the academic literature and existing fielded technologies for fuel marking and tracking. These intensive surveys were followed by initial experimental assays of promising marker candidates. From an early field of four to six candidates, one was chosen. After aging studies proved that marker to be substandard in meeting all our desired expectations for the marker, new chemistries to offer similar or greater optical 
Table 2. Dyes used for fuel marking worldwide

(Source: http://www.wikipedia.com/fueldyes)

\begin{tabular}{|c|c|c|}
\hline Country & Fuel & Dye \\
\hline \multirow[t]{2}{*}{ Ger. Australia } & Regular Unleaded Petrol & Purple (or Bronze) \\
\hline & Premium Unleaded Petrol & Yellow \\
\hline Austria & Heating oil & Any red dye \\
\hline I*I Canada & Agricultural Fuel & Red/purple dye \\
\hline \multirow[t]{2}{*}{ trinland } & Heating oil & Furfural and Solvent Yellow 124 \\
\hline & $\begin{array}{l}\text { Diesel for construction and } \\
\text { agriculture }\end{array}$ & Furfural and Solvent Yellow 124 \\
\hline \multirow[t]{2}{*}{ France } & Gas oil & Solvent Red 24 \\
\hline & Marine diesel & Solvent Blue 35 \\
\hline \multirow[t]{2}{*}{ Estonia } & Heating oil & Automate Red NR or similar + Solvent Yellow 124 \\
\hline & Agricultural diesel & Automate Blue 8 GHF or similar + Solvent Yellow 124 \\
\hline Germany & Heating oil & Solvent Yellow 124 and similar \\
\hline \multirow[t]{2}{*}{ 玩 Greece } & Heating oil & Any red dye \\
\hline & Marine diesel & Any black dye \\
\hline \multirow{2}{*}{ Ireland } & Gas oil & Solvent Blue 79 \\
\hline & Kerosene & Solvent Red 19 and similar \\
\hline \multirow[t]{2}{*}{ Italy } & Heating oil & Solvent Red 161 \\
\hline & Gas oil & Solvent Green 33 \\
\hline Netherlands & Agricultural diesel & Any red dye and the additive Furfural \\
\hline ع & Agricultural diesel & Any green dye \\
\hline \multirow[t]{2}{*}{ (3) Portugal } & Agricultural diesel & Solvent Blue 35 \\
\hline & Heating oil & Solvent Red 19 and similar \\
\hline \multirow[t]{2}{*}{ Spain } & Agricultural diesel & Any red dye \\
\hline & Heating oil & Any blue dye \\
\hline Eweden & Heating oil & Solvent Blue 35, Solvent Blue 79, Solvent Blue 98 \\
\hline \multirow[t]{2}{*}{ 인 United Kingdom } & Gas oil (“Red Diesel”) & Solvent Red 24, quinizarin \\
\hline & Rebated kerosene & Coumarin \\
\hline European Union & Many rebated & Solvent Yellow 124 (“Euromarker”) \\
\hline 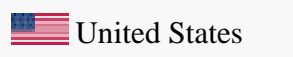 & Low-tax fuels, high-sulfur fuels & Solvent Red 26, Solvent Red 164 \\
\hline \multirow[t]{4}{*}{ Worldwide } & Aviation gasoline 80/87 & Red dye \\
\hline & Aviation gasoline 82UL & Purple dye \\
\hline & Aviation gasoline 100LL & Blue dye \\
\hline & Aviation gasoline 100/130 & green dye \\
\hline
\end{tabular}


response accompanied by increased chemical stability were identified. Out of this effort came the discovery of SiNaph as a solution for FTE project fuel marker. A discussion of current fuel additives as well as a description of the chemicals in diesel fuel that attacked early marker follows. This information helps frame where SiNaph falls in the scheme of fuel additives, and what it means for it to withstand acid attack and thereby maintain a stable optical response over the course of months. Both these aspects relate directly back to the requirements set forth above for a successful fuel marker moiety.

\subsubsection{Required Marker Characteristics: Compatibility as a Fuel Additive}

Nickel and vanadium, among other earthen metals, are inherent in the raw petroleum sources that can contaminate refined diesel and foul automotive mechanisms and the environment over time. More metals can be introduced during fuel production and storage, such as copper, iron, nickel, and zinc. In addition, other components can accumulate within engines or in emissions that result from fuel-performanceimproving additives. The ORNL FTE project team evaluated these levels of metals, inorganic compounds, and larger organic molecules to illustrate the fact that SiNaph adds no insult to the engine or environment outside the bounds of existing fuel and oil contents. Table 3 lists common additives to diesel motor fuels. Fuel additives, generally speaking, comprise chemical substances added to gasoline, diesel, kerosene, and other fuels to impart or improve certain properties. Acetone, ether, nitrous oxide (nitrous), nitromethane (nitro), butyl rubber, ferox, oxyhydrogen, ferrous picrate, silicone and other anti-foaming agents, and tetranitromethane, fuel injector cleaners, fuel injector treatments, octane boosters, diesel fuel treatments, and water removers are also available. Additional categories of fuel additives include antioxidants, hybrid compound blends, oxygenates, antiknock agents, leader scavengers, fuel dyes, metal deactivators, and corrosion inhibitors. Both nitrous oxide (nitrous) and nitromethane (nitro) are used in auto racing and other high-performance applications. Acetone is a vaporization additive used mainly with methane racing fuel. Butyl rubber is a detergent for diesel fuel injectors, while ferox is an engine catalyst that improves fuel economy and reduces emissions in gasoline engines. Ferrous picrate and oxyhydrogen are also used to improve fuel mileage. Silicone is used as anti-foaming agent in diesel engines. Fuel additives such as tetranitromethane can help to improve the combustion properties of diesel fuel. Finally, lubricants can, over time, migrate into fuel lines and, when burned, build up components like zinc and silicon, which are deposited into the diesel particulate filter. After calculating the contribution of silicon from SiNaph to a diesel particulate filter over time, and in consultation with diesel particulate filtration (DPF) and lube oil researchers within ORNL, it was determined that the levels of silicon or silicon dioxide resulting from SiNaph marking of diesel fuel were adequately low to fall within the range of inorganic oxide deposition due to lube oil and engine wear.

Table 3. Diesel motor fuel additives

\begin{tabular}{lll}
\hline \multicolumn{1}{c}{ Purpose } & \multicolumn{1}{c}{ Identity } & \multicolumn{1}{c}{ Amount } \\
\hline Ignition improvers & n-hexyl nitrate & $0.2 \%$ \\
Stability improvers & Peptizing agents ${ }^{a}$ (can be acidic or basic) & $100 \mathrm{ppm}$ \\
Corrosion stabilizers & Sulfonates & $50 \mathrm{ppm}$ \\
Conductivity improvers & Polymeric amine salt & Not listed \\
Winter de-icer & Ethanol & $0.1 \%$ \\
\hline
\end{tabular}

${ }^{a}$ Peptizing agents enhance dispersion of a substance into colloidal form and contributes to colloidal stability once formed. These are surface-active molecules added to a suspending medium to promote uniform and maximum separation of extremely fine solid particles, often of colloidal size.

As an extended example of the levels of silicon, which was our main concern for accumulation in the engines of vehicles burning diesel marked with SiNaph, it is highlighted in the ISO 8217 parameters in Table 4 that aggregate aluminum and silicon much be at $80 \mathrm{ppm}$ or below. In our marked diesel, silicon is at 800 parts per billion (ppb), an order of magnitude lower than this example. This lent early 
encouragement to the utility of the SiNaph marker system, although marine fuels were not the primary target for SiNaph marking.

Table 4. Some parameters of marine fuel oils according to ISO 8217 (3rd Ed., 2005)

\begin{tabular}{|c|c|c|c|c|c|c|}
\hline \multicolumn{7}{|c|}{ Marine residual fuels (RMA30-RME 180 denote different grades) } \\
\hline Parameter & Unit & Limit & RMA 30 & RMB 30 & RMD 80 & RME 180 \\
\hline Density at $15^{\circ} \mathrm{C}$ & $\mathrm{kg} / \mathrm{m}^{3}$ & Max & 960.0 & 975.0 & 980.0 & 991.0 \\
\hline Viscosity at $50^{\circ} \mathrm{C}$ & $\mathrm{mm}^{2} / \mathrm{s}$ & Max & 30.0 & 30.0 & 80.0 & 180.0 \\
\hline Water & $\% \mathrm{~V} / \mathrm{V}$ & Max & 0.5 & 0.5 & 0.5 & 0.5 \\
\hline Sulfur $^{a}$ & $\%(\mathrm{~m} / \mathrm{m})$ & Max & 3.5 & 3.5 & 4.0 & 4.5 \\
\hline Aluminum + Silicon $^{b}$ & $\mathrm{mg} / \mathrm{kg}$ & Max & 80 & 80 & 80 & 80 \\
\hline Flash point $^{c}$ & ${ }^{\circ} \mathrm{C}$ & Min & 60 & 60 & 60 & 60 \\
\hline Pour point, Summer & ${ }^{\circ} \mathrm{C}$ & Max & 6 & 24 & 30 & 30 \\
\hline Pour point, Winter & ${ }^{\circ} \mathrm{C}$ & Max & 0 & 24 & 30 & 30 \\
\hline
\end{tabular}

${ }^{a}$ Maximum sulfur content is $1.5 \%$ in designated areas.

${ }^{b}$ The aluminum+silicon value is used to check for remains of the catalyst after catalytic cracking. Most catalysts contain aluminum or silicon, and remains of catalyst can cause damage to the engine.

${ }^{c}$ The flash point of all fuels used in the engine room should be at least $60^{\circ} \mathrm{C}$.

A final evaluation of the SiNaph as compatible in engines did not come until deep into the marker-sensor characterization when a sensitivity determination could be made and the linear dynamic range of the total system was defined. Several days of effort, including searches of commercial and scientific literature as well as consultation with fuel scientists within ORNL and with contacts in the commercial fuel blending sector, concluded that the amount of silicon contributed to the engine from this marker system at $1 \mathrm{ppm}$ ( $\sim 60 \mathrm{ppb}$ silicon) concentration dye in diesel is well below what is introduced by lubricating oil and other external sources, and therefore poses no threat to engine performance or emissions characteristics. Once the level of $800 \mathrm{ppb}$ of SiNaph was established as a detectable concentration for fuel marking with the current sensor system, a calculation was executed to determine that the amount of silicon dioxide produced over 100,000 miles driven by a 20 mile per gallon vehicle is $1.405 \mathrm{~g}$. The levels of silicon contributed by the SiNaph are well within a range comparable to other inorganic materials that are used in fuel and lubricant oil additives such as zinc. This finding was promising in the process of moving the fuel marker system into a potential position of commercialization.

\subsubsection{Required Marker Characteristics: Utility as a Tracking Moiety}

The identification of an appropriate fuel taggant began with an extensive literature searches to determine which dyes or tags are already being utilized in the field, both in the United States and abroad. This serves multiple purposes, including

- identifying which wavelength ranges our own chosen taggant will have to avoid to mitigate interference from diluent dyes and

- familiarizing the research team with the various chemistries employed to achieve dye-fuel miscibility, engine compatibility, and environmental impact.

Several dyes were identified to include the most probable diluent fuel dyes based on usage in untaxed diesel fuel in the United States; as indicated above, the selected taggant must fluoresce in a range outside that of these existing dyes. These dyes included Solvent Red 26 (agricultural diesel), Solvent Yellow 124, also known as EuroMarker (some marine and heating fuels in the United States, all transportation diesel in the European Union), Solvent Orange 7 (some fuel ethanol in United States and all fuel ethanol in Brazil), and Solvent Blue 59 (marine diesel in United States). Aviation fuel in the United States is dyed 
green or purple; green is generally based on a cyanine dye and purple from a mix of blue anthraquinone and red azo dyes, in most cases. In the process of identifying these dyes, a survey was made of similar dyes from Sigma-Aldrich to evaluate their ranges of absorption of light in order to estimate orthogonality with existing, potentially interfering fielded diesel dyes. One common characteristic among existing fuel dyes included that the dye molecule should be a neutral organic and not contain metallic moieties, which could lead to engine reactivity and corrosiveness or shifts in spectral response due to chemical complexation or intramolecular rearrangement. The most prevalent dye structures are shown in Fig. 4, both for fielded dyes and markers novel to fuel marking used in the FTE project (but not ultimately selected due to degradation, as discussed later).

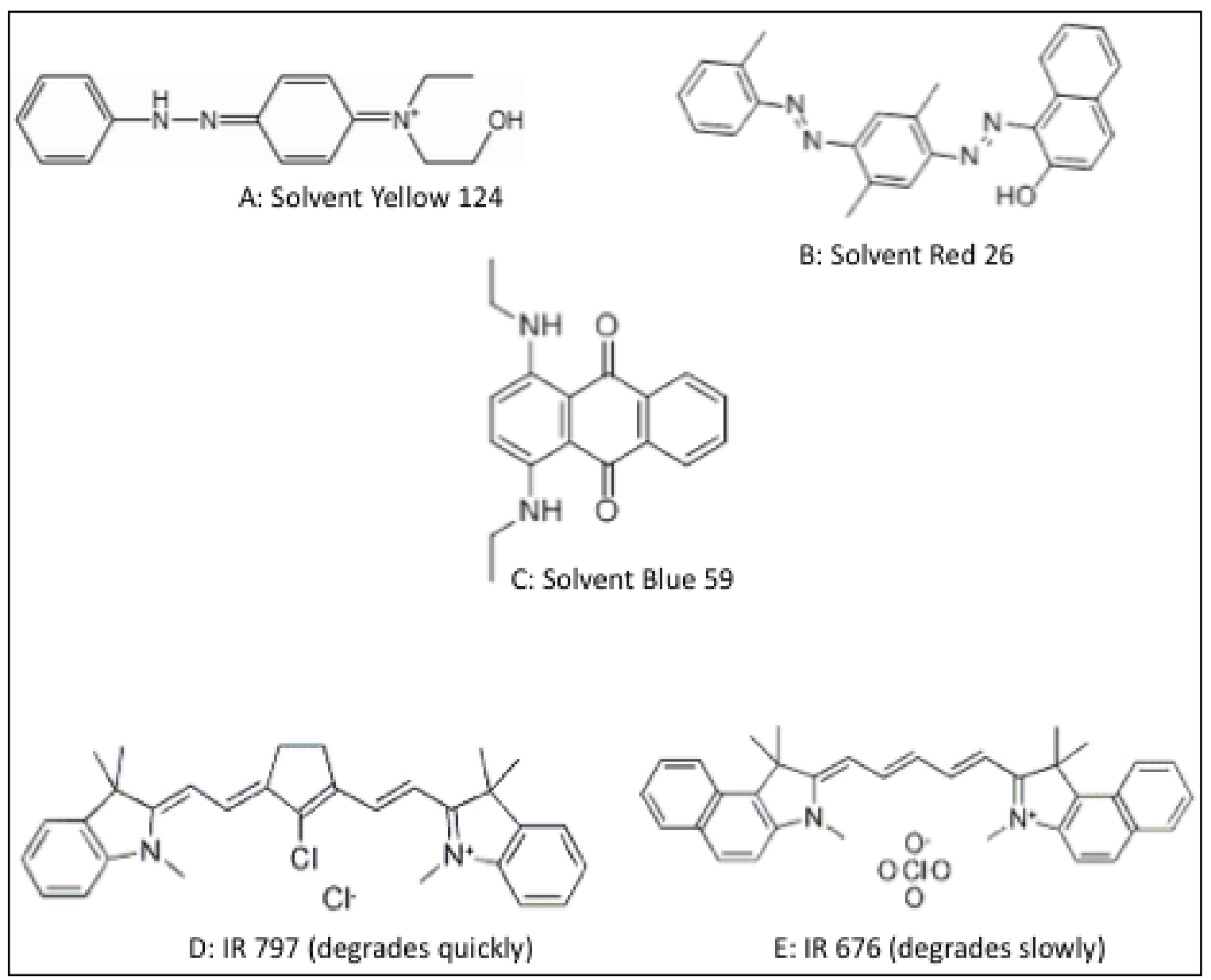

Fig. 4. Some representative structures of several prominent fielded fuel dyes (A-C) and candidate dyes tested but not selected for the fuel tax evasion project (D and E).

Standard samples of the already-fielded dyes listed above were ordered to allow UV-visible absorbance and fluorescence measurements to be made on them in the concentrations commensurate with use in pump diesel. Stock solutions for spectral interrogation were spiked to contain $7 \mathrm{ppm}$ dye in diesel. This concentration matches the levels of Solvent Yellow 124 used in transportation diesel in the European Union and strikes a balance between the 3 ppm Solvent Blue 59 used in marine and aviation diesel and the 15 ppm Solvent Red 26 used in agricultural diesel here in the United States. Moreover, these stock solutions were mixed into unmarked diesel at 3\% and 30\% "untaxed" loading, for example, to simulate a situation where someone had diluted taxed pump diesel with as high as $1 / 3$ and as low as $1 / 33$ volumes of untaxed (dyed) diesel. Absorbance and fluorescence data described in detail below is shown in Fig. 5 through Fig. 7. 


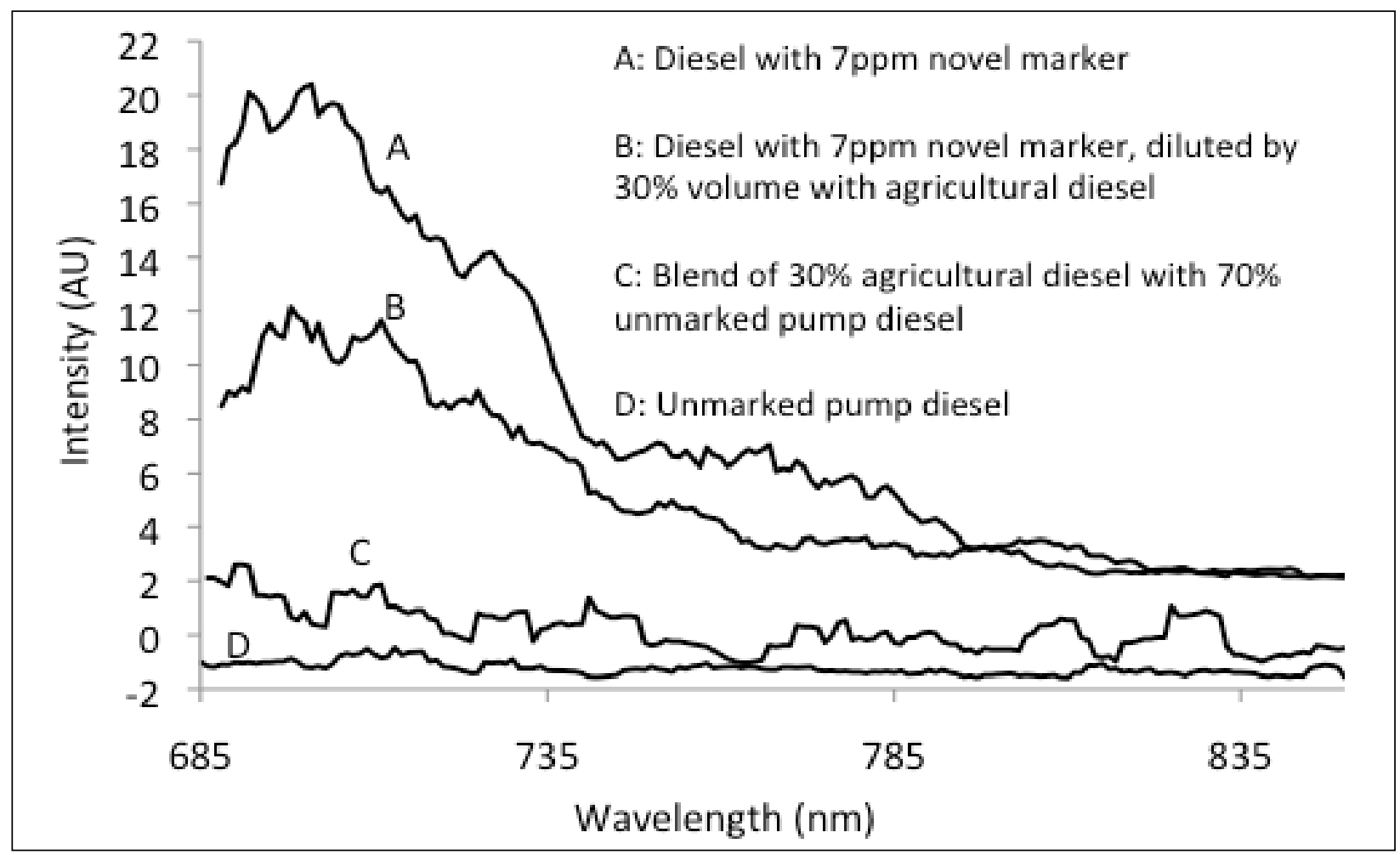

Fig. 5. Absorbance measurements with IR676 ("novel marker”) and agricultural diesel demonstrating lack of interference with fuel-tax-evasion project long wavelength marker’s spectral region by Solvent Red 26/164based agricultural diesel dye.

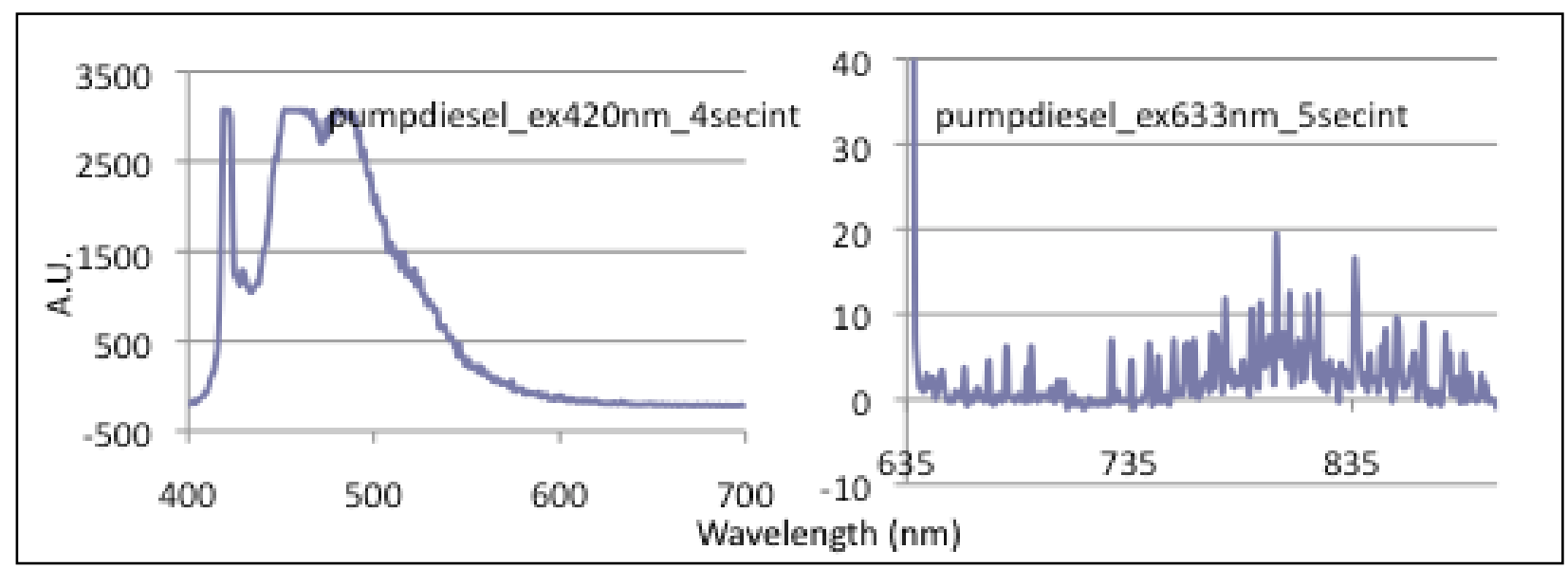

Fig. 6. Fluorescence spectra collected for pump diesel to determine whether competing fluorescence in the wavelength region of excitation above $630 \mathrm{~nm}$ would pose any significant interference, and none was noted. 


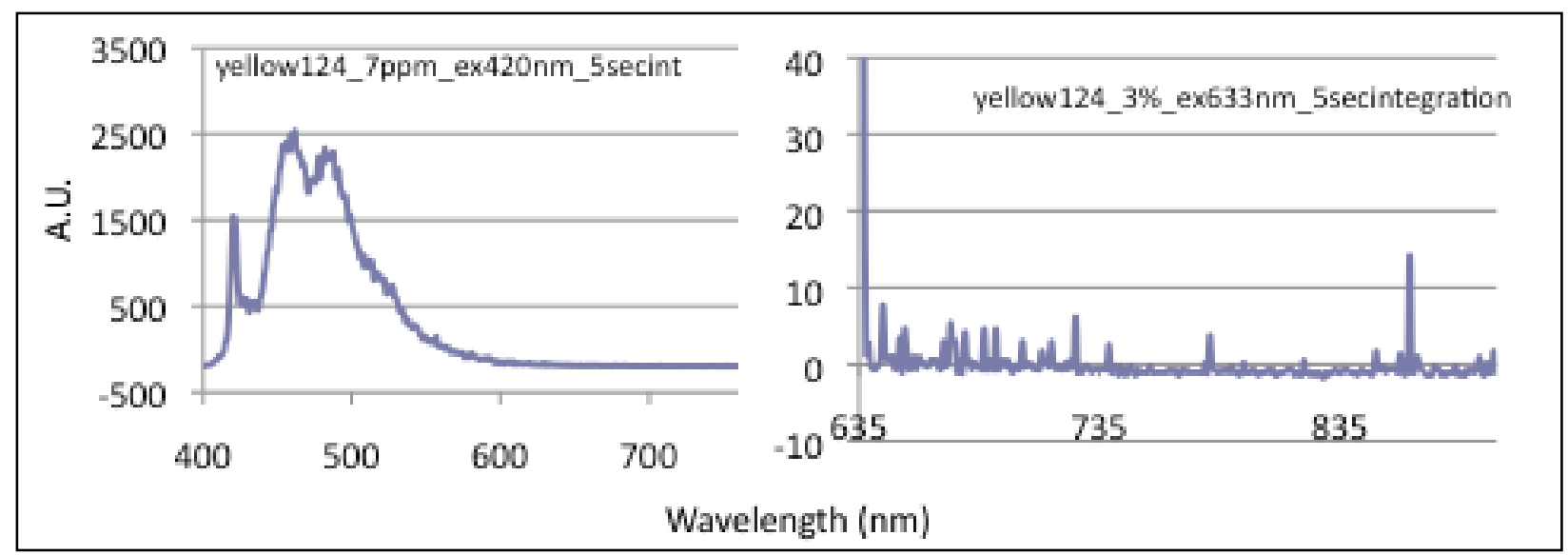

Fig. 7. Fluorescence spectra collected for pump diesel containing the fielded fuel dye Solvent Yellow 124 to determine whether competing fluorescence in wavelength region of excitation above $630 \mathrm{~nm}$ would pose any significant interference, and none was noted.

Specifically, each compound or mixture of isomers from a list of seven potential dye markers emissive beyond $650 \mathrm{~nm}$ and novel to fuel applications was tested for the following characteristics:

- $\quad$ solubility in a suitable delivery solvent (acetone, methanol, ethanol, hexane; all are acceptable based on environmental and engine performance parameters);

- $\quad$ miscibility of marker/delivery solvent stock solution with diesel at a range of mass loadings spanning 15 ppb to 28 ppm;

- $\quad$ appreciable fluorescence emission as tested on a benchtop fluorimeter using both the manufacturer-reported maximum and absorbance maxima experimentally determined during excitation and emission scans; and

- ability to discerning the exact wavelengths of greatest emission due to a particular excitation energy was important to maximizing the efficiency of dye sensing since these dyes have never been tested in hydrocarbon or diesel matrices. (Excitation and emission energies can shift slightly due to solvent environment.)

Two candidate dye markers were down-selected from the list of seven based on their solubility and emissive performance, and each of these was tested for altered optical output due to interference from agricultural diesel, which contains a non-fluorescent red dye. No interference was measured for pump diesel sample containing $0.3 \mathrm{ppm}$ novel marker, $1.5 \mathrm{ppm}$ novel marker, and $7 \mathrm{ppm}$ novel marker for a range of dilutions by agricultural diesel $(0.5 \%, 5 \%, 10 \%, 15 \%$, and $30 \%$ volume dilution by agricultural diesel in each of the three marked diesel concentrations). Figure 5 shows that the only change effected by dilutions by agricultural diesel is the decrease in the broadband fluorescence response of the marker IR676 centered at $\sim 695 \mathrm{~nm}$. This was expected due to the dilution itself, and no dimerization or quenching effects of the novel marker's emission results from the introduction of the red dye molecules with agricultural diesel mixing, again as shown in Fig. 5. Note that addition of agricultural diesel reduces (and is not additive to) the absorbance in the magnitude of its dilution of the IR676 marked diesel.

To determine the level of fluorescence interference we could expect from the fielded dye standards in diesel, fluorimetry (quantitative measurement of the fluorescent response over a given spectral range) was carried out on these samples in pump diesel. These data are shown in Fig. 6 and Fig. 7 with descriptive titles on each panel. After several iterations of excitation of pump diesel, it was determined that any illumination below 575-600 nm generated some fluorescent background from the diesel fuel alone as well 
as from the Solvent Yellow 124 at $7 \mathrm{ppm}$. Next, an excitation wavelength of $633 \mathrm{~nm}$ was chosen, which is commensurate with widely available, respectably high-powered and low-cost helium-neon gas lasers $(632.8 \mathrm{~nm})$ and red diode lasers $(635 \mathrm{~nm})$. None of the currently fielded dyes or the pump diesel itself showed any significant fluorescent response to the $633 \mathrm{~nm}$ wavelength excitation. These data are included in Appendix A. This held true for the $690 \mathrm{~nm}$ line that was ultimately chosen as well. These early marker tests and literature survey gave us a significant direction for specifying a taggant and detection system.

After this survey, it was determined that a long visible or near-infrared wavelength of excitation would serve best to avoid interference from existing diesel and fuel dye fluorescence; therefore, a marker chemistry to facilitate fluorescence in that range was sought accordingly. During this decision-making period, the Fuel-in-Oil (now $\mathrm{DaFiO}{ }^{\circledR}$ ) laser-induced fluorescence spectroscopy system was discussed with one of its ORNL inventors, with the Sensor Team and Fuel Marker Team, for issues such as sensor drift, optic clouding, and signal to background. The decision was made to focus on laser-induced fluorescent direct detection of a high-fluorescence quantum yield diesel marker. A more complete description of the advantages, basic detection components, and specific requirements for this project of laser-induced fluorescence (LIF) is provided in the sensors section of this report. Briefly, using a laser to excite fluorescence of a dye taggant in a region of the electromagnetic spectrum (in our case, the visible spectrum above $630 \mathrm{~nm}$ ) where the diesel and other diluent dyes show little to no emissive properties, as can be seen in the fluorescence emission scans in Fig. 7, lends an advantage in sensitivity to the detection technique. Ultimately, it was also decided by the sensors team that a spectral ratio method would give the most reliable quantitation of the marker level. Two wavelengths, mutually exclusive of detecting diesel and the marker, were identified. One measures diesel fluorescence at $405 \mathrm{~nm}$ excitation, and one measures marker fluorescence with excitation in the high $600 \mathrm{~nm}$ range. Early testing of the IR676 marker indicated such a scheme would function, and the spectral evidence of mutual exclusivity is shown in Fig. 8.

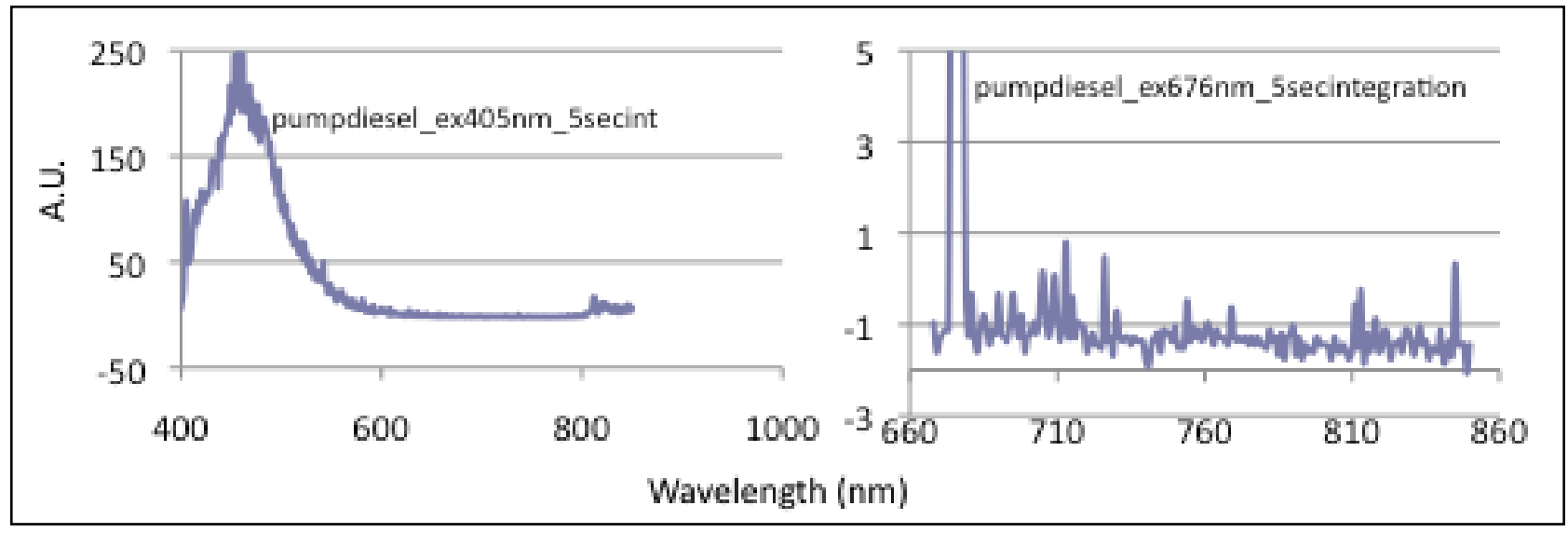

Fig. 8. Diesel fuel is excited with $405 \mathrm{~nm}$ light (left) and $676 \mathrm{~nm}$ light (right).

Although the exciting radiation is visible in these spectra, the lack of significant fluorescent emission at $676 \mathrm{~nm}$ relative to $405 \mathrm{~nm}$ indicated that a ratiometric spectral quantitation would work well with this system; that is, differences between different concentrations of the taggant would be discernable with good resolution.

After a general approach and wavelength ranges desirable for the marker emission were identified, a broad range of dye concentrations in diesel were tested for the linearity of their fluorescence responses on the benchtop commercial fluorimeter and, later, on the self-referencing prototype built by the Sensor Team. This broad characterization shown in Fig. 9 indicated two pertinent results: 


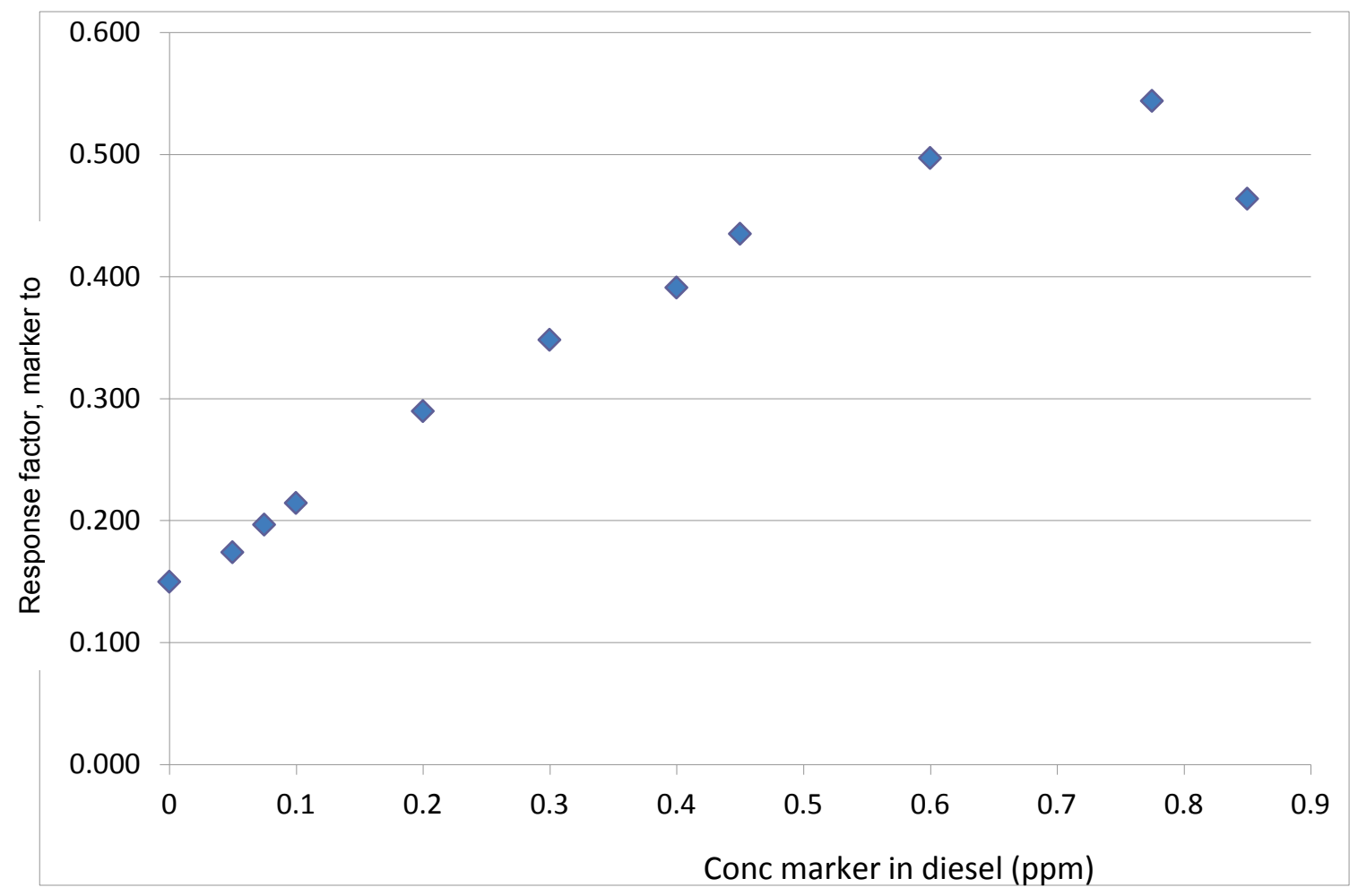

Fig. 9. Broad range calibration of IR676 in diesel on the prototype self-referencing fuel marker sensor.

- a useful range over which the dye should be tested using much smaller incremental concentrations was identified, and

- the self-referencing fuel marker sensor prototype was shown to be functional, and at least an order of magnitude more sensitive than the laboratory benchtop fluorimeter.

This result is not surprising in view of the fact that lasers and bandpass filters are used instead of a broadly emissive lamp and dispersive gratings, as in the benchtop system. Lasers offer higher total power at wavelengths more centered at the relevant energy for dye and diesel excitation, and judiciously selected filters increase throughput at chosen wavelengths where the response is strongest with less loss of total power due to dispersion in a grating.

Next, a range of concentrations was tested within the linear region of the concentrations probed during the linear dynamic range investigation. These data are shown in Fig. 10. This set of experiments was carried out to more closely monitor change in signal with change in amount of dye added on the self-referencing prototype built by the Sensor Team. Solutions were prepared by mass via serial dilution and measured in a cuvette; the whole series (ascending order) was measured three times. This focused-range characterization indicated two pertinent results:

- a useful range over which the dye should be tested using much smaller incremental concentrations was identified, and

- the self-referencing fuel marker sensor prototype was confirmed to be functional, and at least an order of magnitude more sensitive than the laboratory benchtop fluorimeter. 


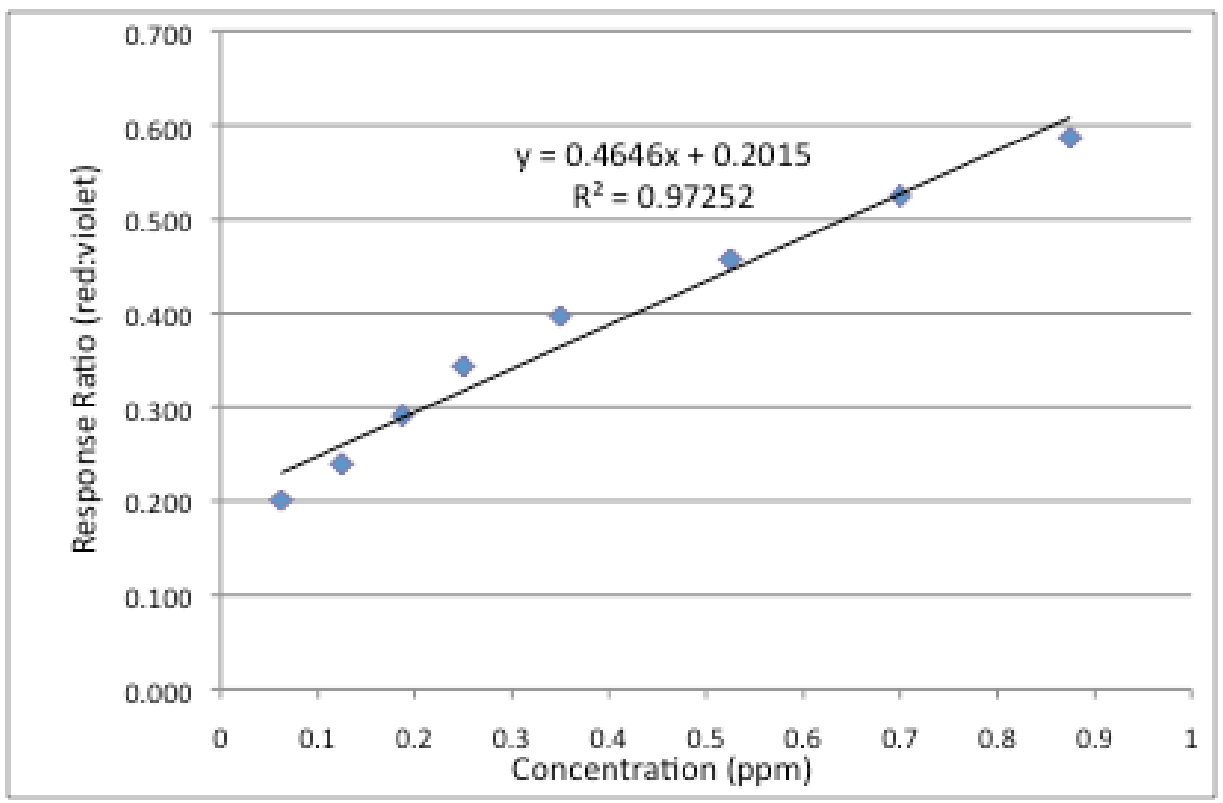

Fig. 10. Focused-range incremental calibration curve of IR676 in diesel on the prototype self-referencing fuel marker sensor.

After fluorescence by a long-wavelength-emitting dye was identified as the detection route of choice for fuel marker dilution monitoring, new dyes were then identified and tested for high fluorescent yield in diesel and longer-term stability. These included molecules with similar structural characteristics to the previously tested compounds, in the hope that the favorable diesel solubilities observed with the degradable dye would remain. These candidates were surveyed over a period commensurate with duration of marker persistence desired by the user community. The experiments were conducted using the independent laboratory fluorimeter as well as the modified benchtop prototype laser-induced fluorescence detection system. In this manner, two goals, (1) persistent marker identification and (2) preliminary prototype validation, can be accomplished nearly simultaneously.

\subsection{AGING AND CHEMICAL STABILITY TESTING}

\subsubsection{IR676}

Samples of diesel fuel spiked with various amounts IR676 dye were examined by absorption and emission spectroscopy to follow the fate of the dye with time. Figure 11 shows the absorption spectra of samples containing 0.052 ppm IR676, 0.079 ppm IR676, 0.11 ppm IR676, 9.4 ppm IR676, and one "blank” sample, containing no dye, taken on March 30, 2010.

As the data indicates, only the sample containing 9.4 ppm IR676 shows a broad peak around 500-750 nm, indicative of dye absorption. Figure 12 shows the emission spectra of all the samples excited at $639 \mathrm{~nm}$ taken on March 30, 2010.

It is clear from the graph that samples with less than 1 ppm IR676 hardly emit in the region where IR676 emits. However, sample 84-8, which contains 9.4 ppm IR676, shows a good emission. Figure 13 shows the emission spectra of all the samples taken on April 7, 2010.

As can be seen from the graph, besides the $9.4 \mathrm{ppm}$ sample, the samples all show practically no emission in the region where IR676 emits. Figure 14 shows the changes in the absorption spectra of the samples with time taken on April 13, 2010. 


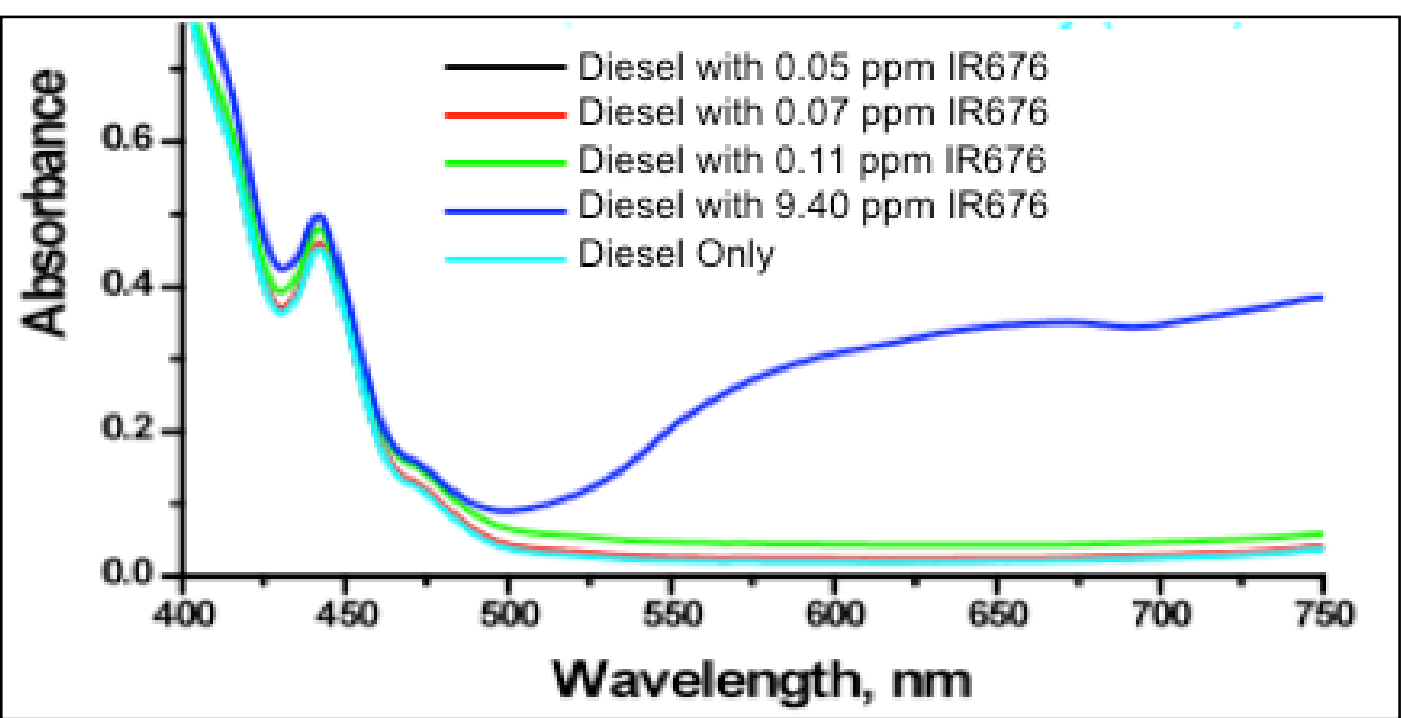

Fig. 11. Absorption spectra of IR676 at concentrations ranging from $0.052 \mathrm{ppm}-9.4 \mathrm{ppm}$ collected after no wait time.

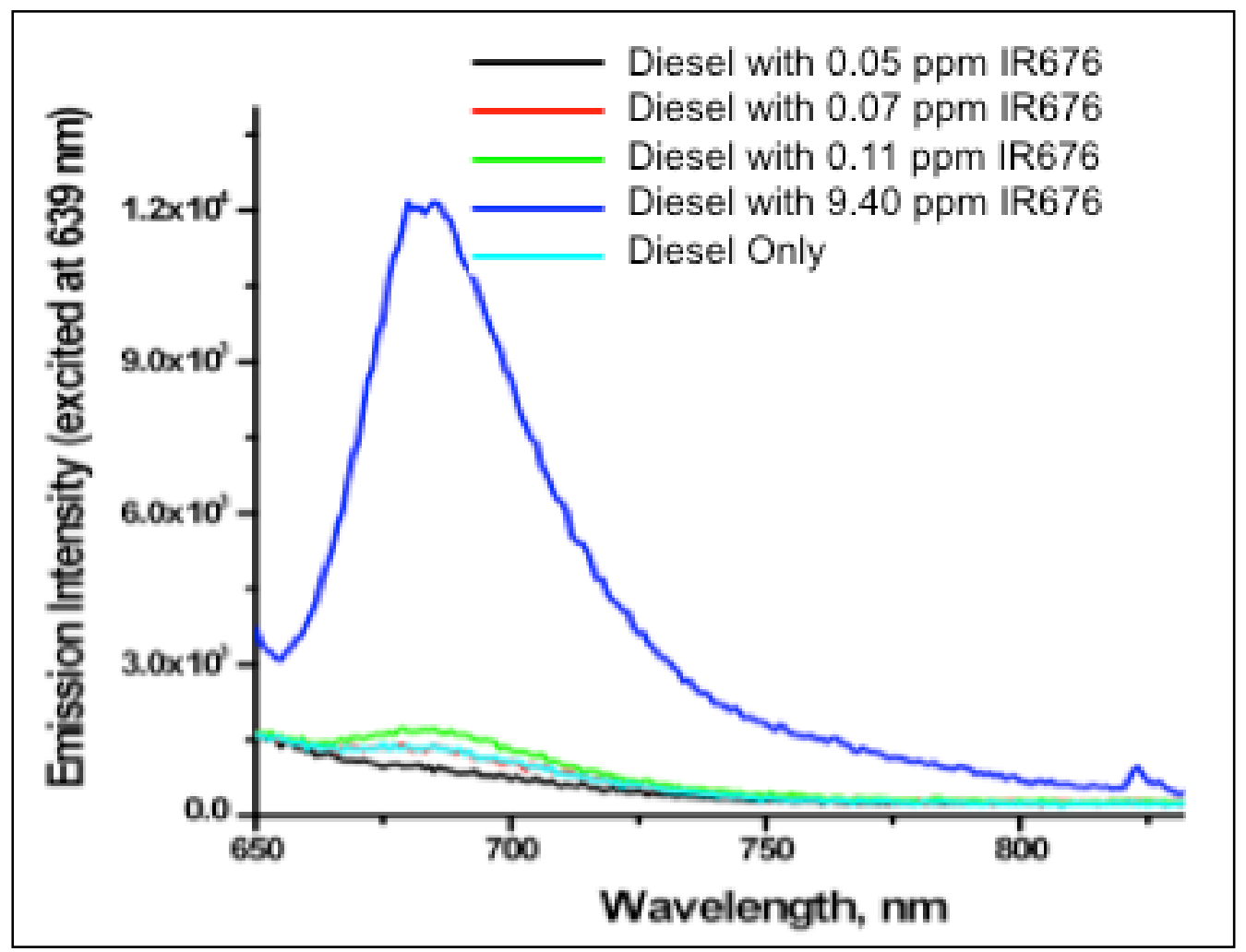

Fig. 12. Emission spectra collected after no wait time of IR676 samples in diesel. 


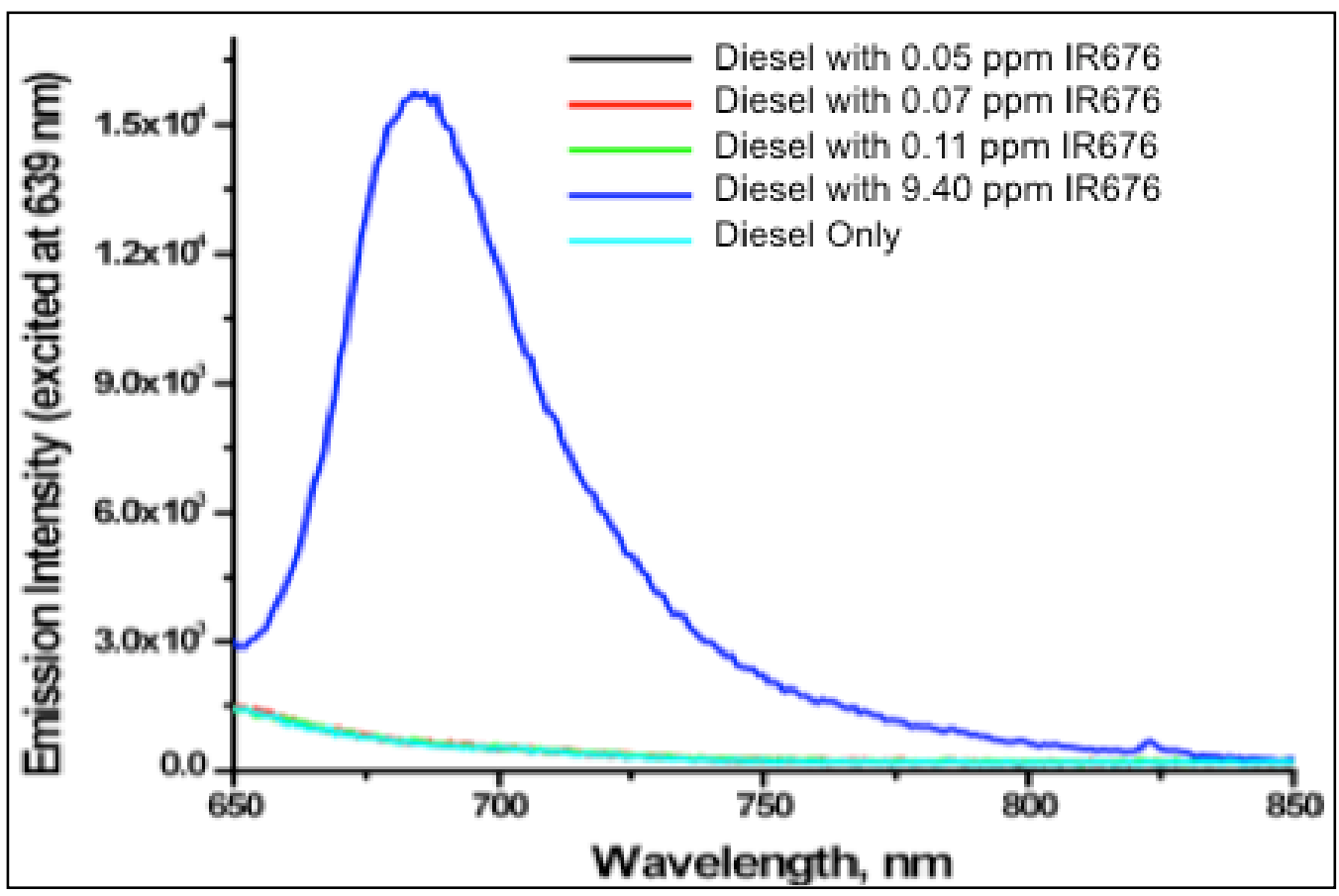

Fig. 13. Emission spectra collected after 1 week of aging of IR676 samples in diesel.

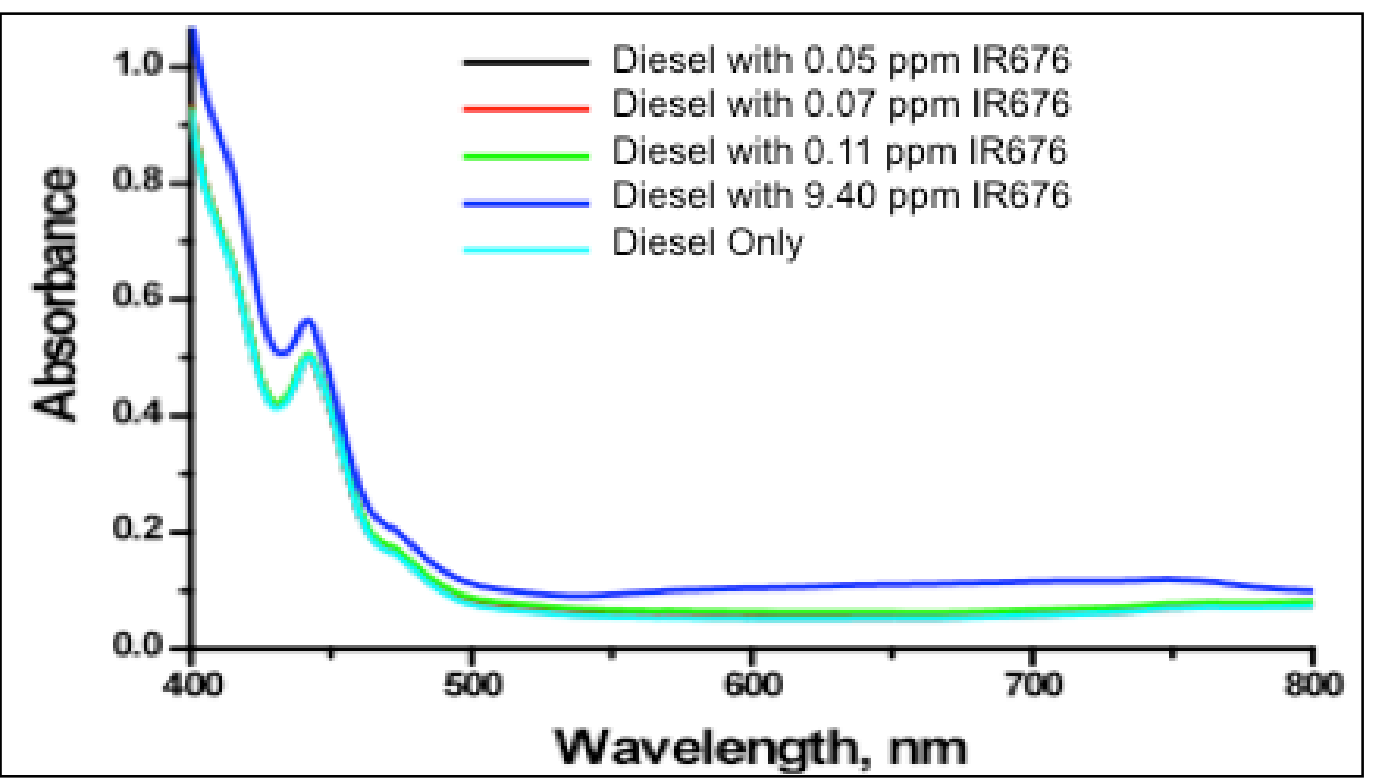

Fig. 14. Absorption spectra after 2 weeks of IR676 aging in diesel fuel. 
Comparison of Fig. 14 with Fig. 11 reveals that only the absorption of the sample containing $9.4 \mathrm{ppm}$ IR676 in the 500-750 $\mathrm{nm}$ region has decreased considerably. The emission spectra of the samples taken on April 13, 2010, are shown in Fig. 15.

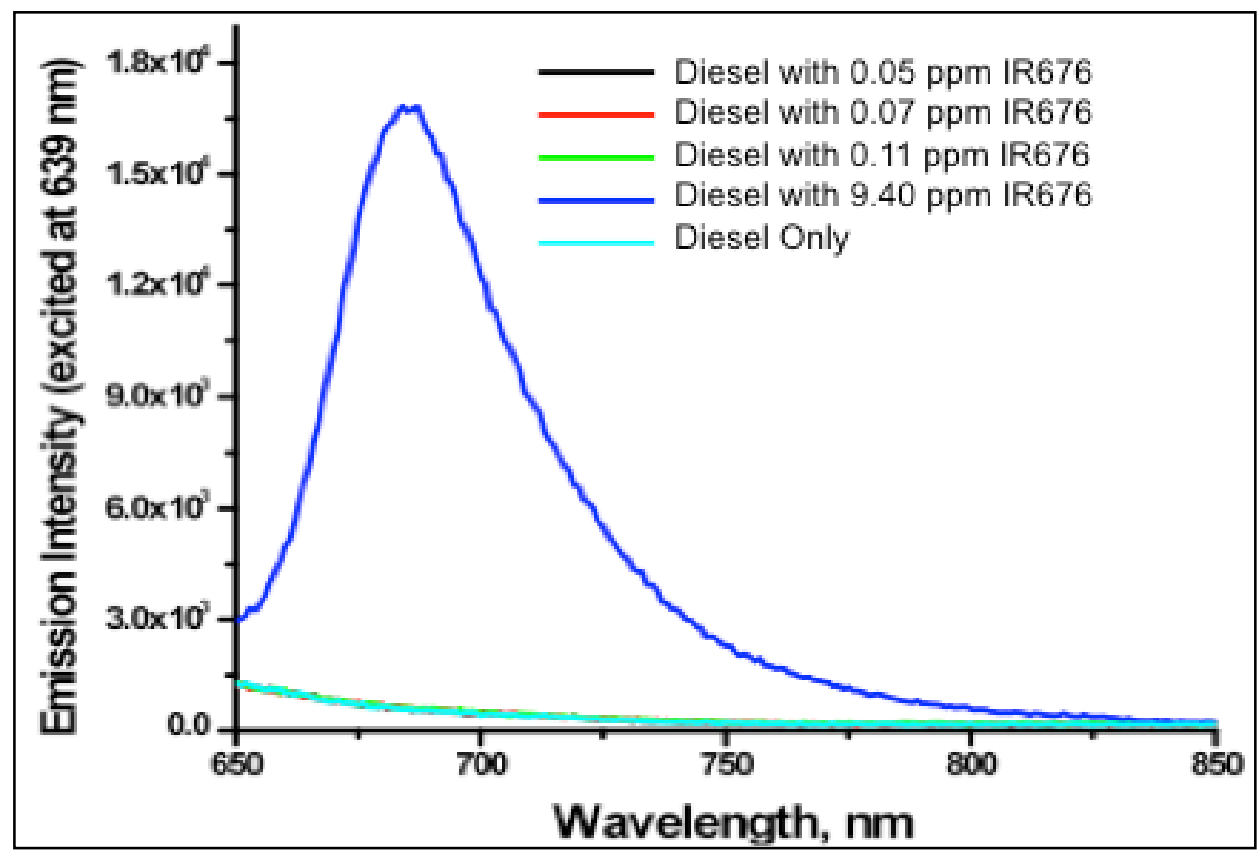

Fig. 15. Emission spectra collected after 2 weeks of aging of IR676 samples in diesel.

It is clear from the data that only the sample containing 9.4 ppm showed a significant emission, and all the other ones show none. Interestingly, the emission of the $9.4 \mathrm{ppm}$ sample appears to have increased with time, as shown in Fig. 16.

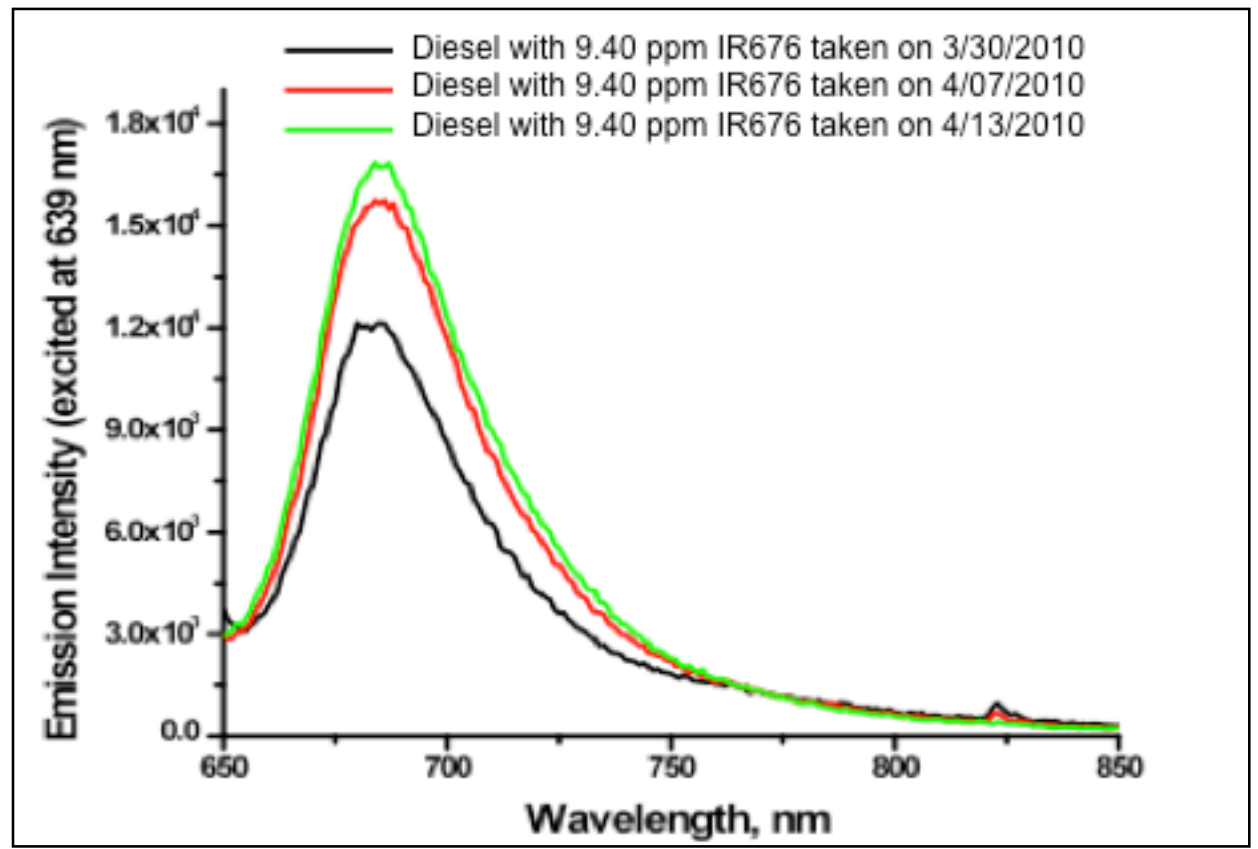

Fig. 16. Change in emission spectrum of over time for the highest concentration of IR676 in diesel. 
This increase may be due to slow dissolution of IR676 at higher concentrations in diesel fuel. The sample containing 9.4 ppm IR676 was checked finally at an aging time of 3 weeks (April 20, 2010) and showed an emission decrease to $<20 \%$ of the max signal detected on April 13, 2010. This indicated that, even at this higher dye content of the marker, degradation in the diesel occurred.

In Fig. 16, note the slight increase over short time range in fluorescence signal. This is also a disadvantage, since the reading of marker signal must be stable over time to create accurate dilution reporting by the optical marker sensor.

New dyes were then identified and tested for longer-term stability. These included molecules with similar structural characteristics to the original system, in the hope that the favorable diesel solubilities observed with the degradable dye will remain. These candidates were surveyed over a period commensurate with duration of marker persistence desired by the user community. The experiments were conducted using the independent laboratory fluorimeter as well as the modified benchtop prototype laser-induced fluorescence detection system. In this manner, two goals, (1) persistent marker identification and (2) preliminary prototype validation, were accomplished nearly simultaneously.

Building on the experimental evidence that the diesel fuel dye marker system based on the IR 676 dye aged more than desirable for the project objectives, several new dyes were tested over the course of a month. The dye systems surveyed included IR 780; IR 797; IR 806; IR 813; IR820; 3,3-diethylthiatricarbocyanine iodide; silicon 2,3-naphthalocyanine bis(trihexylsilyloxide), hereafter (referred to as SiNaph); and an advanced nanomaterial called a squaraine rotaxane, shown in Fig. 17. This SRfluor ${ }^{\circledR} 680$ is a phenyl crystalline powder, lipophilic squaraine rotaxane analog that emits in the farred region of the spectrum and is known to accumulate at lipophilic sites inside living cells. Spectral properties include an absorbance maximum at $650 \mathrm{~nm}$ and an emission maximum at $678 \mathrm{~nm}$. This marker promised the chemical stability, miscibility with hydrocarbons, and long-wavelength-region activity of a complex ligand system but was ruled out because $1 \mathrm{mg}$ costs $\$ 162.50$.
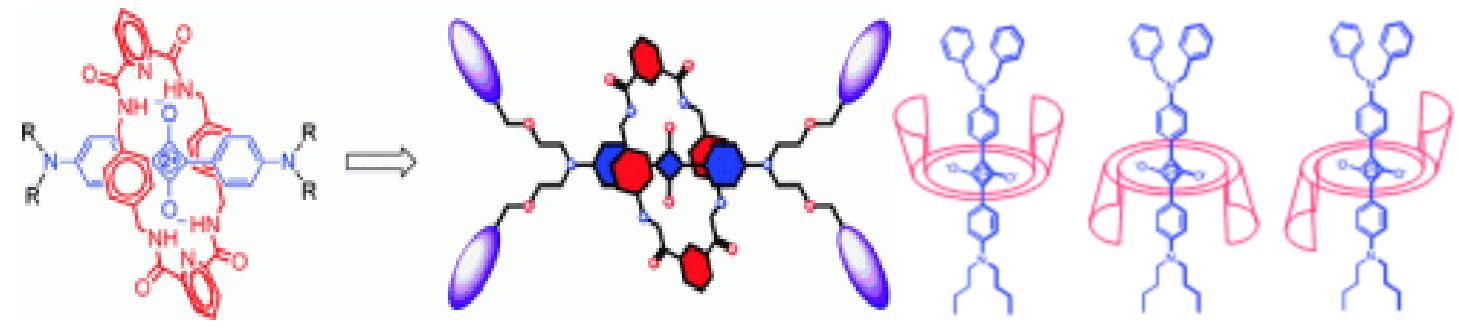

Fig. 17. Various representations of an advanced macromolecule evaluated after stability failure of IR676 but ruled out due to price of $\$ \mathbf{1 6 2 . 5 0}$ for 1 milligram.

Only the penultimate compound mentioned in the above list and discussed throughout this section of the final report, SiNaph, shows long-term stability on the order of tens of weeks and falls anywhere near a workable price. Similar aging to that in the case of the original IR 676 dye was observed over a period of 4 hours to several days. For all other dye systems followed, depending upon the particular dye system spiked into pump diesel, the components of diesel, both intrinsic and additive, were evaluated with a view to what might be compromising the dye emission. It was determined that residual carboxylic acids from additive peptizing agents as well as naphthoic and naphthenic acids (described further below in extended quote and in Fig. 18) and simple fatty acids in the diesel led to the degradation reaction. To test, dyes that were degraded by diesel over a period of days were prepared in acetone or methanol at $10 \mathrm{ppm}$ and spiked with acetic acid or palmitic acid in separate samples, each at $10 \mathrm{ppm}$. These simplified dye/acid

*Price quoted by Molecular Targeting Technologies, Inc, West Chester, Pennsylvania, June 30, 2011. 
contaminant surrogate samples were followed with absorbance measurements confirmed the hypothesis that acids in diesel were the root cause of emission degradation in all cases but that of SiNaph.

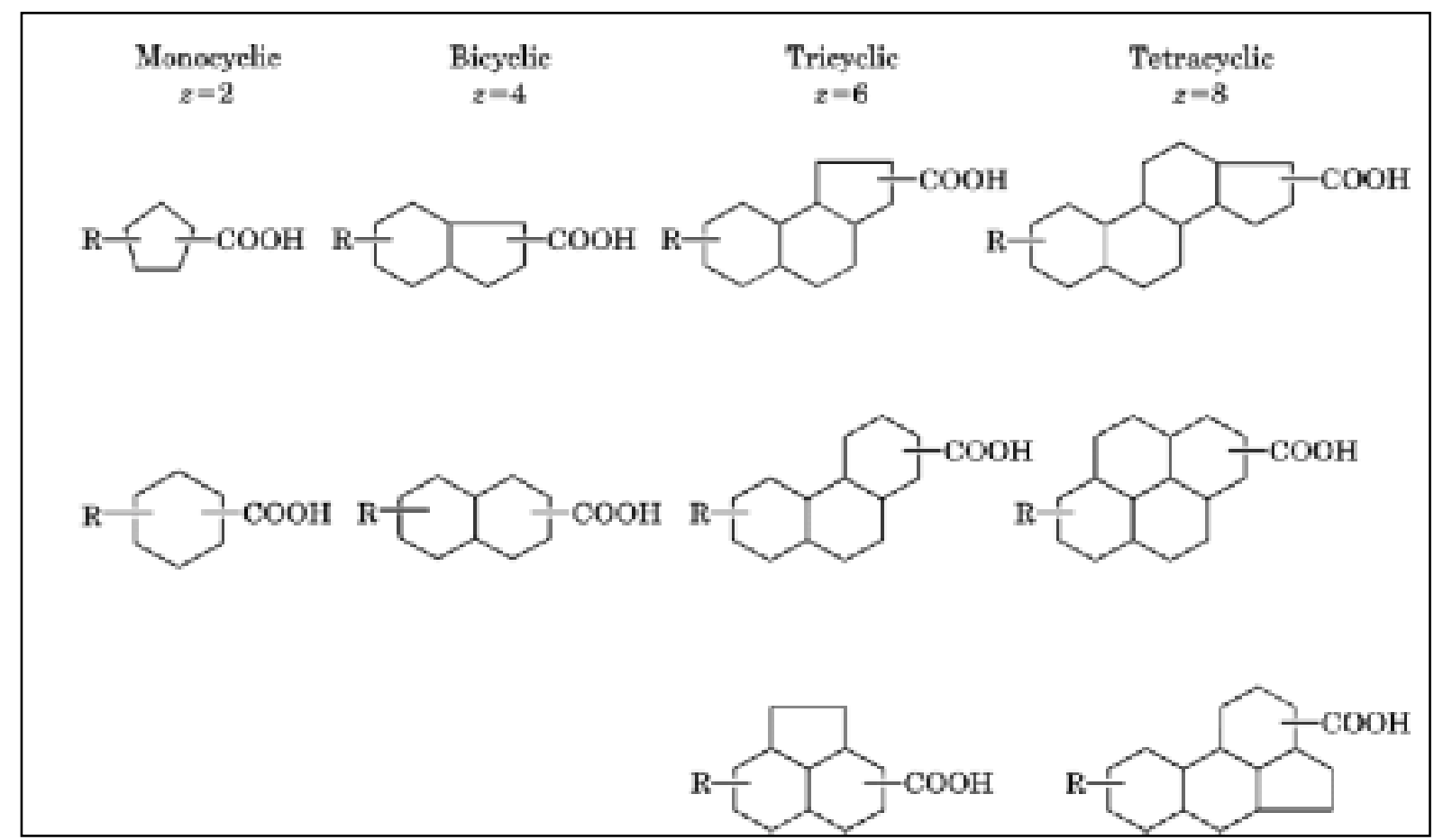

Fig. 18. Naphthenic acids are prevalent in diesel and other heavier hydrocarbon fuels. Shown are typical naphthenic acid structures, where $\mathrm{R}=$ alkyl. For the acyclic case, $\mathrm{z}=0$ and the structure is simply R-COOH.

What follows is an eloquent and concise discussion of naphthenic acids, shown in Fig. 18, intrinsic in diesel fuel.

The following excerpt is from the Kirk-Othmer Encyclopedia of Chemical Technology. ${ }^{1}$

Chemically, naphthenic acids behave like typical carboxylic acids with similar acid strength as the higher fatty acids. Dissociation constants are on the order of $10-5$ to 10-6 (15). They are slightly weaker acids than low molecular weight carboxylic acids such as acetic, but are stronger acids than phenol and cresylic acid. Whereas the principal use of naphthenic acids has been in the production of metal salts, they also can react to form esters, amine salts, amides, imidazolines, and many other derivatives (see Esters, organic; Amides). Derivatives of naphthenic acid often have advantageous physical properties over fatty acid derivatives, particularly their high stability to oxidation and solubility in hydrocarbons. Naphthenic acid corrosion has been a problem in petroleumrefining operations since the early 1900s. Naphthenic acid corrosion data have been reported for various materials of construction (16), and correlations have been found relating corrosion rates to temperature and total acid number (17). Refineries processing highly naphthenic crudes must use steel alloys; 316 stainless steel [11107-04-3] is the material of choice. Conversely, naphthenic acid derivatives find use as corrosion inhibitors in oil-well and petroleum refinery applications. Naphthenic acids occur in a wide boiling range of crude oil fractions, with acid content increasing with boiling point to a maximum in the gas oil fraction (ca $325^{\circ} \mathrm{C}$ ). Jet fuel, kerosene, and diesel fractions are the source of most commercial naphthenic acid. The acid number of the naphthenic acids decreases as heavier petroleum fractions are isolated, ranging from $255 \mathrm{mg} \mathrm{KOH} / \mathrm{g}$ 
for acids recovered from kerosene and 170 from diesel, to 108 from heavy fuel oil (19). The amount of unsaturation as indicated by iodine number also increases in the high molecular weight acids recovered from heavier distillation cuts.

We postulate that these compounds are largely responsible for the degradation of IR 676 and other dyes not workable in the long term for diesel fuel due to acid attack and cleaving of the fluorophore structure. The presence of silicon within the SiNaph prevents permanent acid attack by offering electronic association of the lone pair electrons on the nitrogens, a probable site of association for the naphthenic acids. This association would lead to molecular disintegration or simply form a complex whose spectral qualities are not amenable for fluorescent excitation and emission at the wavelengths outside the instrumental setup.

\subsubsection{SiNaph}

Laboratory testing showed that SiNaph persists with stable absorbance and emission profiles over 6 weeks. SiNaph is also directly soluble in diesel, requiring no additional organic solvent for creation of a stock solution. This will facilitate integration of the dye marker into the commercial setting of diesel distribution. To demonstrate SiNaph's stability, Fig. 19 shows absorbance profiles collected at time points throughout a week for a solution of SiNaph in diesel at 1 ppm. Figure 20 shows the excitation and emission characteristics of SiNaph, which indicate that it shows up in a spectral region free of competition from other components both intrinsic and extrinsic to diesel fuel.

Careful testing for the aging or degradation, if any, of the newly identified fuel marker system based on SiNaph dye was carried out. It was found that over a period of 44 days, no degradation of dye optical activity was measurable. For continuing studies, metal from the trailer of an actual tanker was obtained and cut into manageable pieces for use in an aging/degradation test centered on ascertaining whether anything in the tank metal would facilitate in-tank degradation of the fuel marker. The initial absorbance scan of three samples prepared with 1 ppm SiNaph in diesel containing a piece of tank metal is shown in Fig. 21.

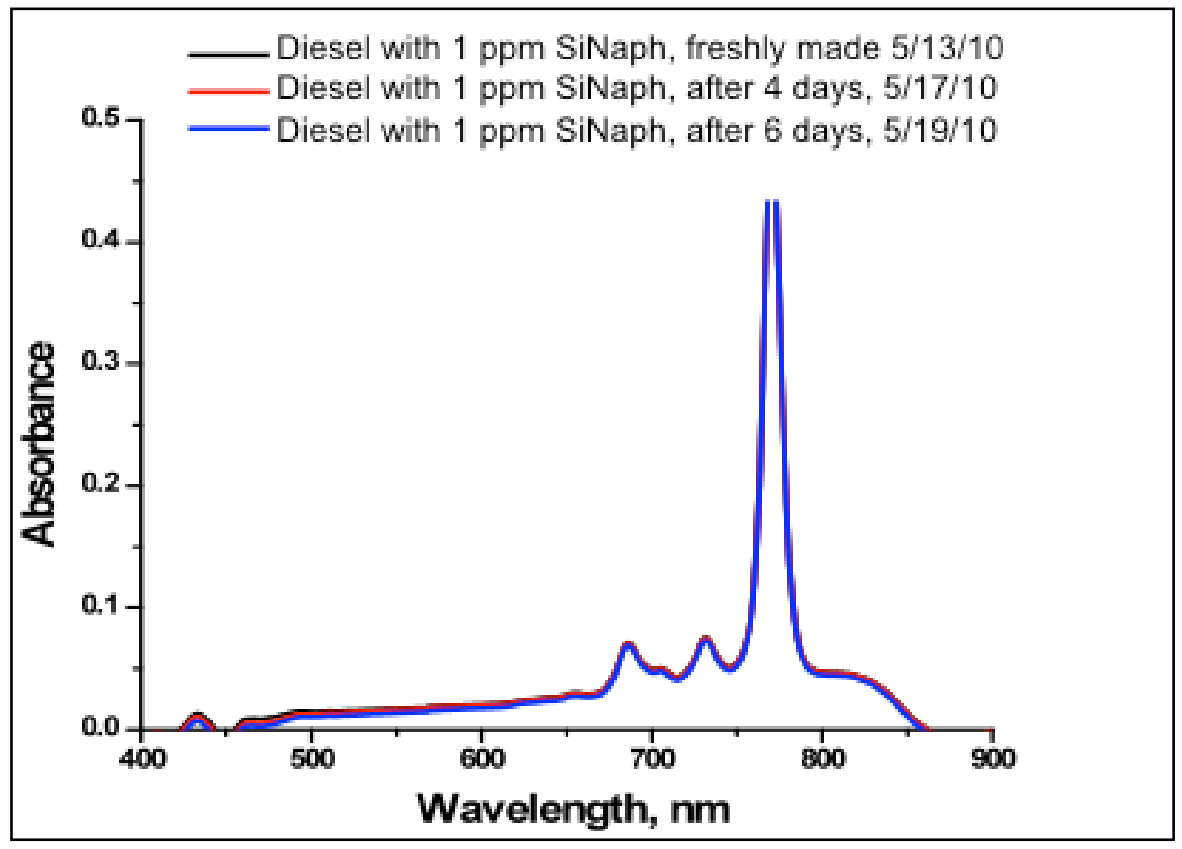

Fig. 19. Absorbance profiles collected over a week’s time for SiNaph. 


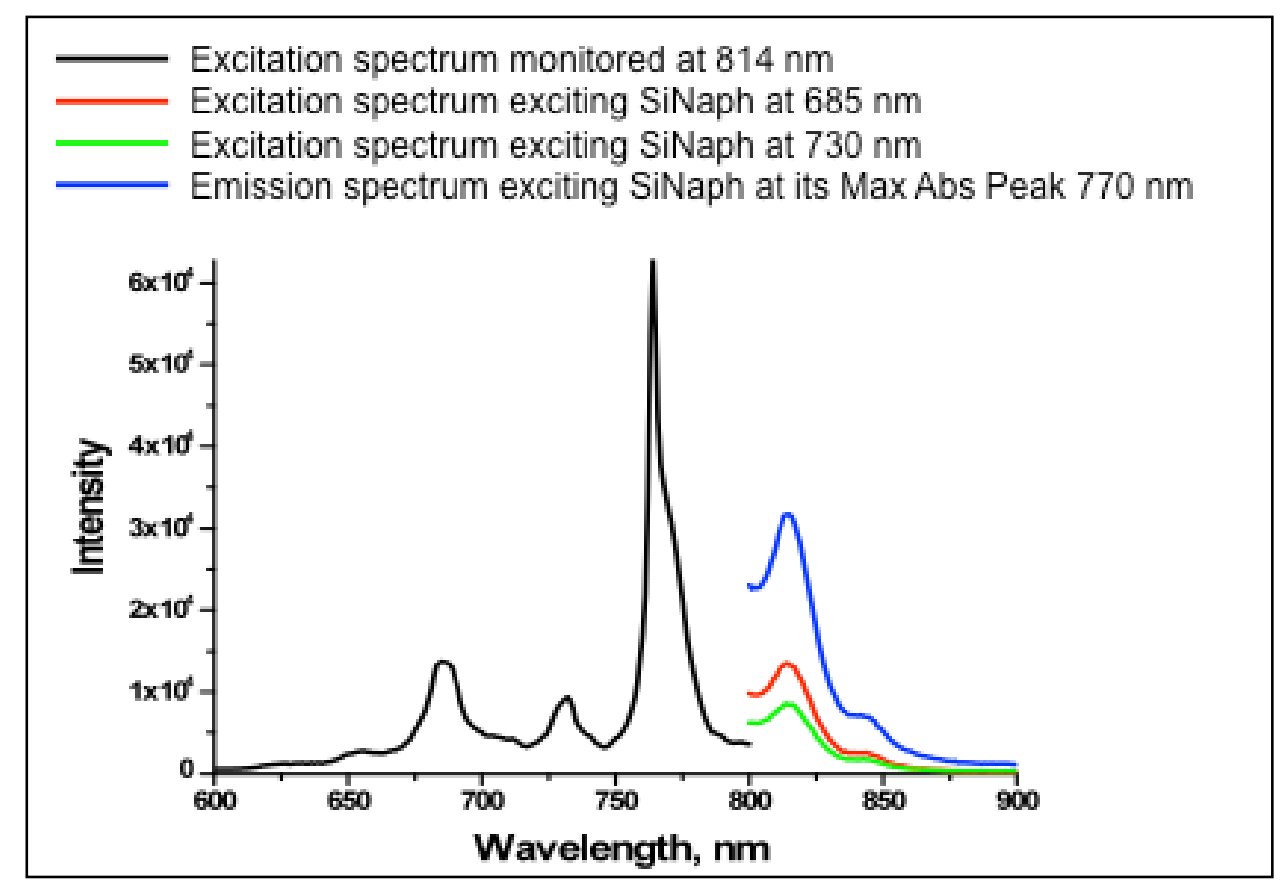

Fig. 20. Excitation and emission characteristics of SiNaph.

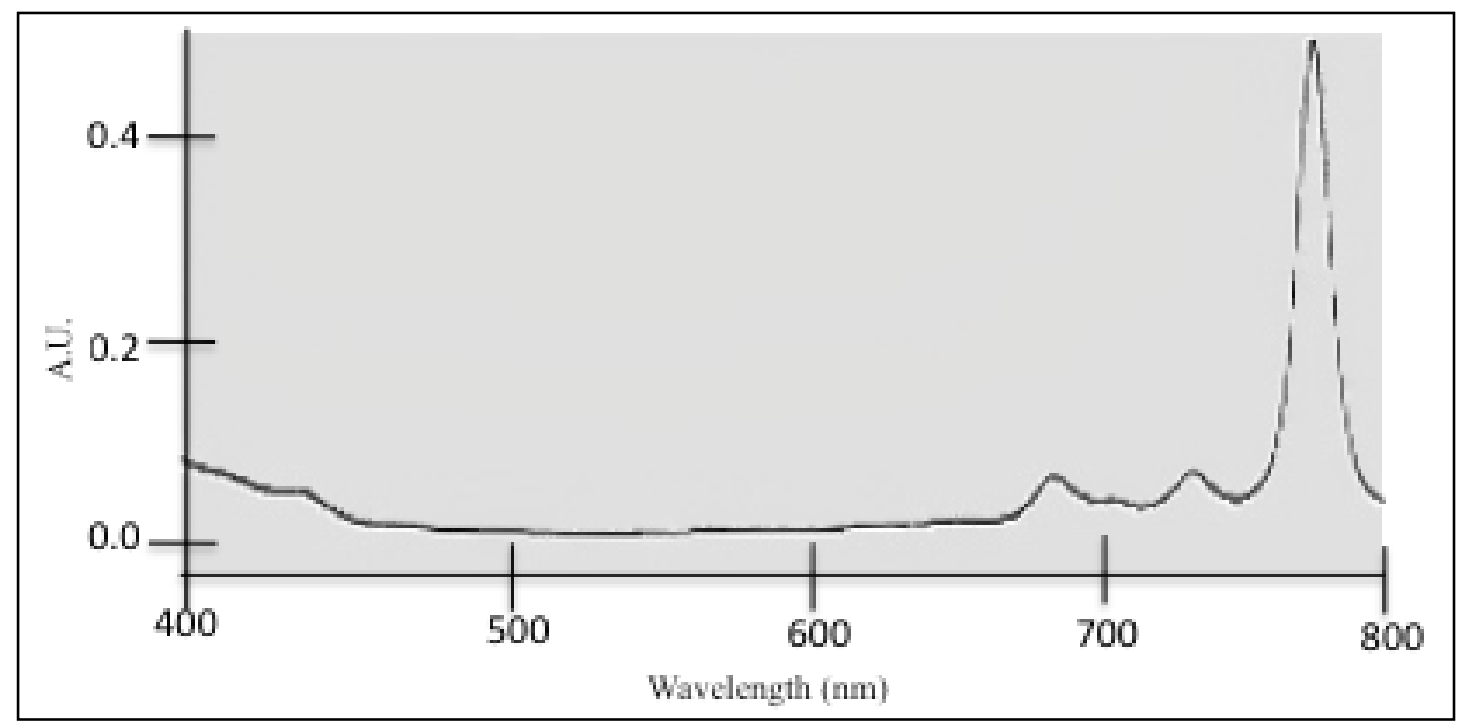

Fig. 21. Initial absorbance measurements at room temperature, low temperature, and high temperature (traces are overlapping) of SiNaph-marked diesel in the presence of tank metal.

One sample remained at room temperature, a second was subjected to freezing temperatures, and a third was heated to $140^{\circ} \mathrm{C}$. These samples continued to be followed spectroscopically to determine whether the presence of metal and/or temperature extremes adversely affected the fuel marker's optical response.

This demonstrated that no immediate effects on the marker result from a reaction with the tank metal and paved the way for longer-term temperature-based studies in the presence of the tank metal. 
After determining that the SiNaph dye is stable in diesel chemical environment after more than 60 days, aging studies were expanded to include temperature variables as well as metal from an actual tanker truck compartment. Inclusion of this metal allowed thermal and temporal aging to proceed in as realistic a chemical environment as possible in light of prior knowledge that metal surfaces sometimes facilitate degradation of organic and ligand-bound chromophores and fluorophores. However, we were able to show that, even over months, the SiNaph retains its optical signature, even at $32^{\circ} \mathrm{F}$ and $>140^{\circ} \mathrm{F}$. It was even found that the optical signature of the fuel marker persisted beyond the lifetime of the diesel itself in the case of the high-temperature time study.

All samples were created as described in the following steps. Aluminum alloy metal cut from a large piece off a fuel tanker truck's tank was machined down to shards measuring 1 in. $\times 2$ in.. One shard was placed in each of five containers, three of which were Nalgene ${ }^{\circledR} 250 \mathrm{~mL}$ jars and two of which were borosilicate glass jars. Pump diesel marked at $1 \mathrm{ppm}$ in SiNaph was added to two of the plastic (Nalgene) bottles and one of the glass jars. The remaining bottle and jar received unmarked diesel to serve as roomtemperature and heated diesel blanks. Initial absorbance spectra were collected against the roomtemperature unmarked diesel blank and then one marked sample went into a freezer, one into a $140^{\circ} \mathrm{F}$ oven, and one onto the lab countertop. The samples were measured every day for a week and every 4 to 7 days after that for 8 weeks. Figure 22 illustrates the stability of the room-temperature aging sample. Figure 23 shows the results at low temperature, and Fig. 24 demonstrates absorbance spectra from the high-temperature samples over time. Figure 25 confirms the degradation of the diesel fuel itself at high temperatures.

Based on observation, diesel degradation is the likely cause of an increasing absorbance both in the 400$500 \mathrm{~nm}$ wavelength regions as well as the eventual creation of a broad, nondescript background as the high temperatures more completely altered the diesel viscosity, hue, and chemical character toward the end of the study. This information was very useful in conveying the longevity of the SiNaph marker system as well as testing the complete sensor suite on the bench and on the test track.

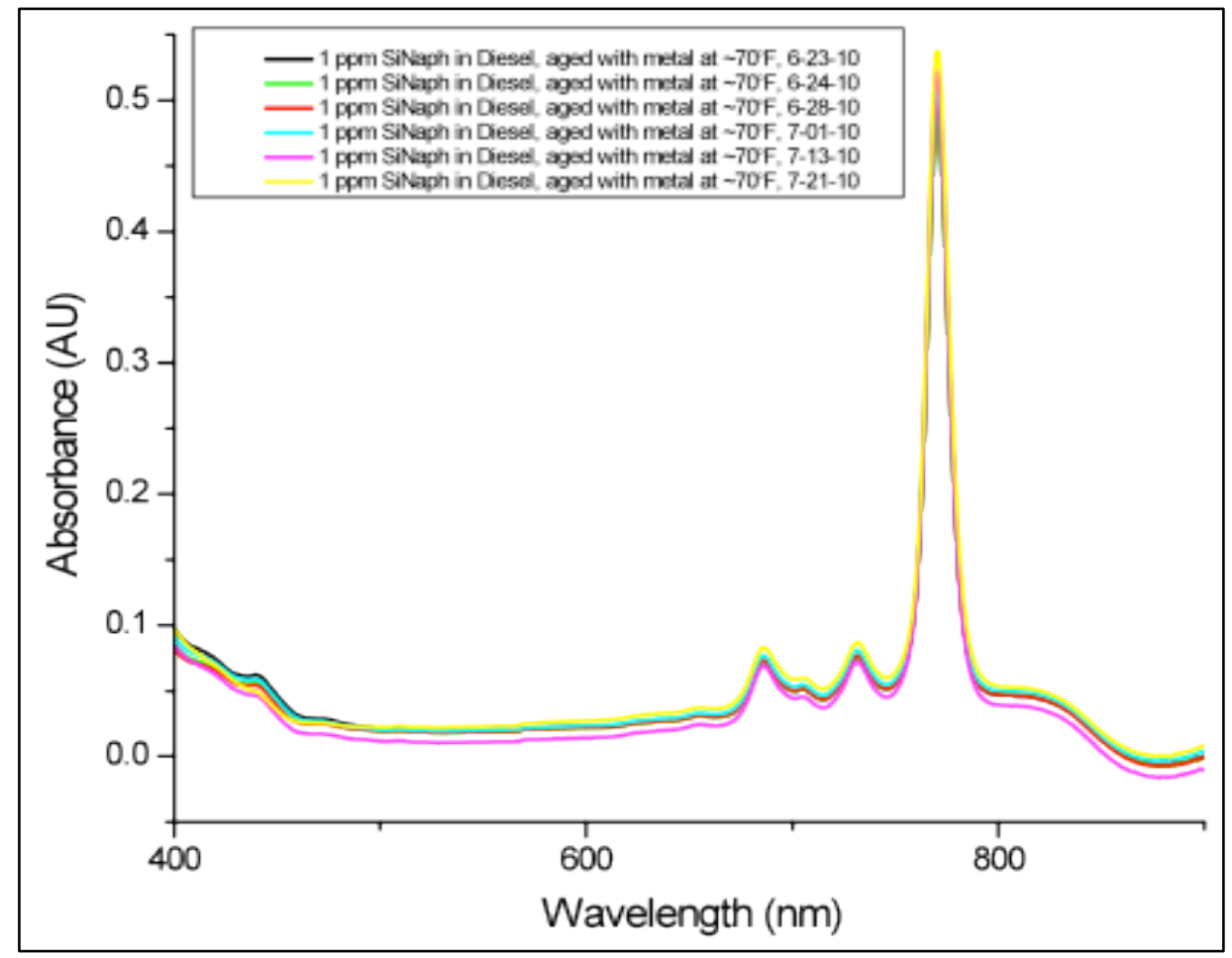

Fig. 22. Room-temperature marked diesel aging study. 


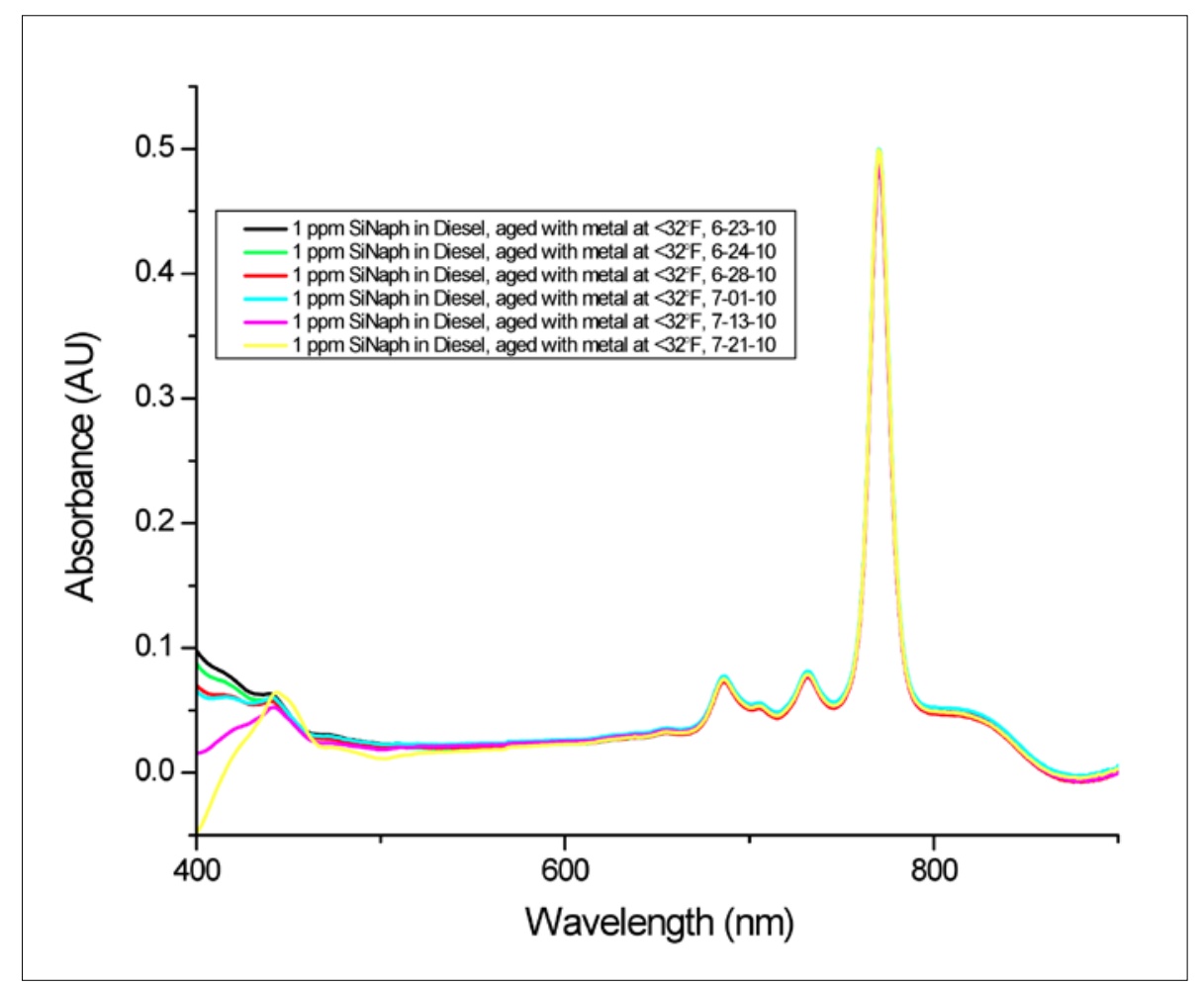

Fig. 23. Low-temperature marked diesel aging study.

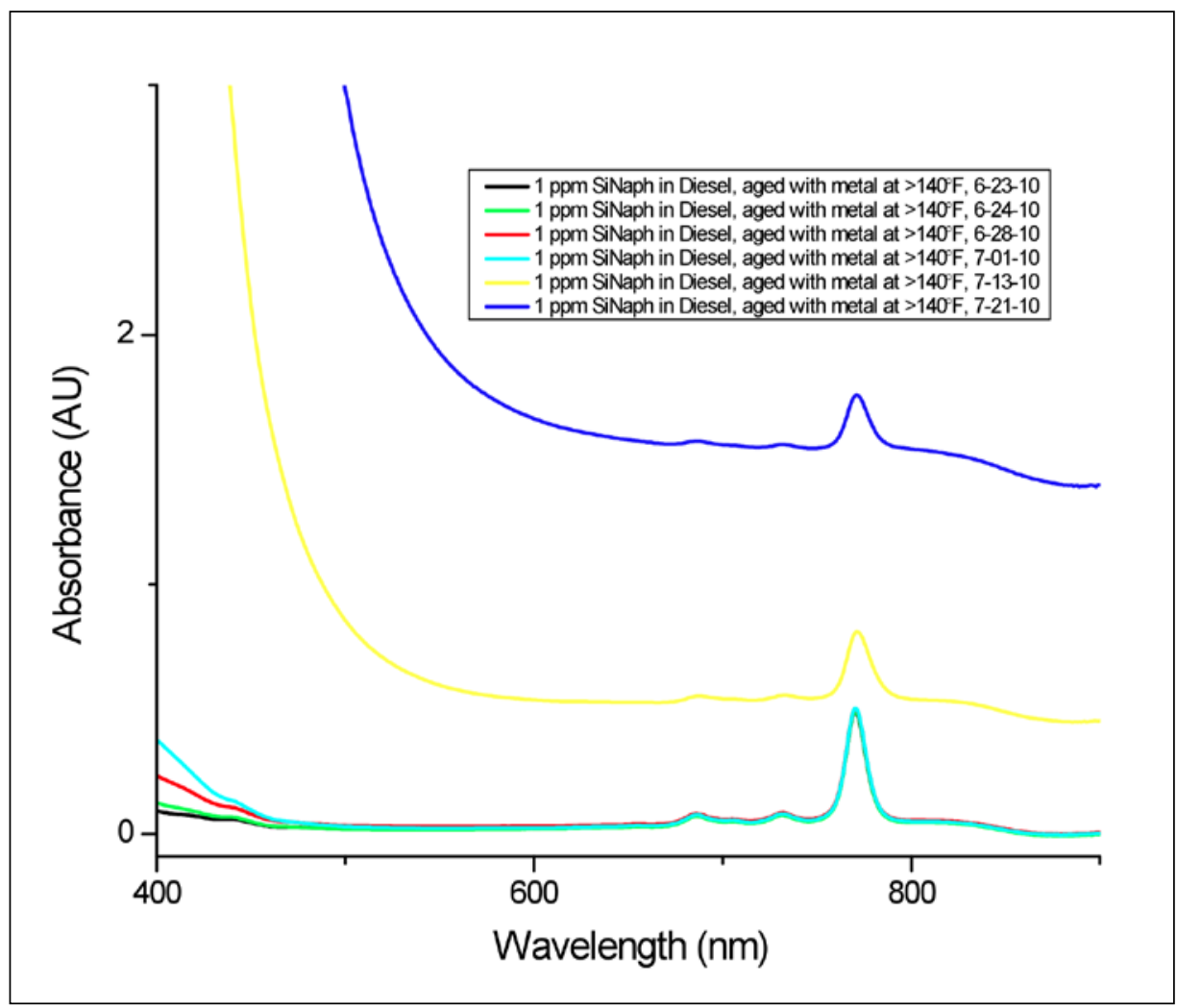

Fig. 24. High-temperature marked diesel aging study. 


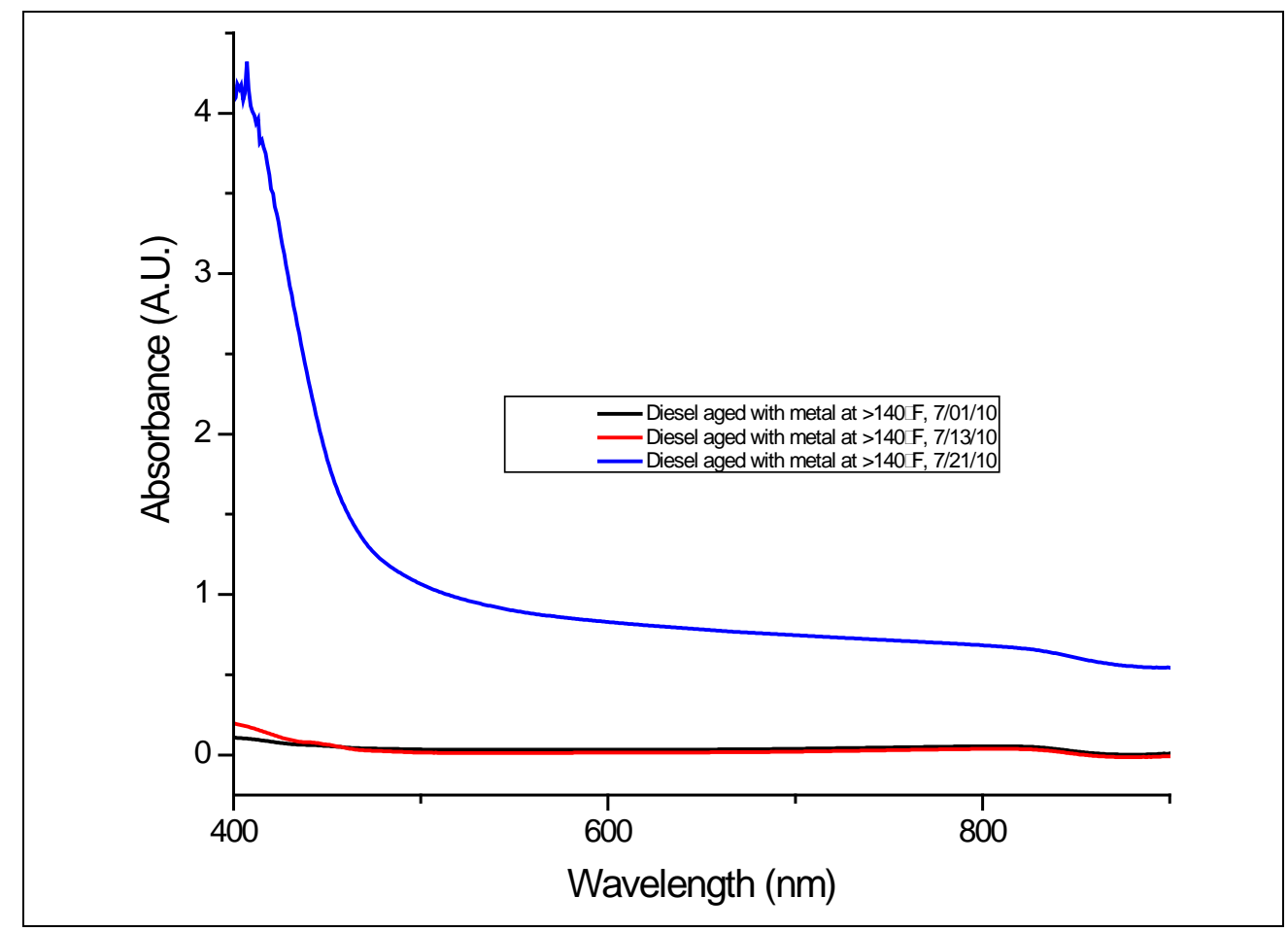

Fig. 25. High-temperature unmarked diesel aging study.

Additionally, further fuel maker aging to a time of even greater than 3 months reveals that even at high temperature $\left(140^{\circ} \mathrm{F}\right)$ in the presence of tank metal, the diesel itself degrades into a yellowed, viscous product that creates an overall higher background for the dye, which only shows a negligible amount of degradation and band broadening.

\subsubsection{Sister Dye: 3,3'-diethylthiatricarbocyanine iodide}

An exhaustive search of literature and commercial chemical starting materials resulted in the identification of a less expensive (by about an order of magnitude) sister dye marker for the detection of fuel dilution and adulteration. The excitation and emission characteristics of this naphthalocyanine moiety are amenable for use with the current SiNaph sensor configuration. The chief structural difference is the exclusion of silicon from the more affordable dye marker; this and other minor structural differences, along with lower single-component molecular purity, result in reduced solubility characteristics into diesel and a requirement to be doped in at a $1.5 \mathrm{ppm}$ level for equivalent fluorescent emission yield. Early results in aging with metal studies at low, room, and high temperatures indicate ample stability for low and room temperatures with some signal loss with time at $120^{\circ} \mathrm{F}$. This reduction in stability with heating could also be due to the absence of a organometallic center. A new dye, 3,3'-diethylthiatricarbocyanine iodide, was assayed that offered lower cost than the SiNaph system. It was observed that the "sister" dye, which does not contain silicon and is nearly an order of magnitude less expensive than the SiNaph system, does show some marginal aging characteristics at room temperature and low temperature, as well as significant aging at very high temperatures. A cost/conditions analysis or discussion will allow us to determine if the value added by the SiNaph's longevity at very high temperatures justifies its higher price. Figure 26 shows the difference in absorbance profile of non-Si dye marker at $1 \mathrm{ppm}$ in diesel after 28 days of storage with tank metal in $120^{\circ} \mathrm{F}$ conditions. The blue trace represents the initial absorbance profile of the dye, whereas the red represents its optical absorbance after long-term high-heat storage. This case represents the worst example of long-term optical performance deterioration; in the low and room-temperature aging studies, and in the short-term high-temperature studies, the non-Si marker 
performs acceptably. Figure 26 illustrates the essence of these aging studies, in that the "sister" marker shows negligible optical response after aging less time and at lower temperatures than SiNaph and was therefore deems unusable for the field trials.

As illustrated in Fig. 26, at low and room temperatures, this less expensive dye marker system shows stability. After evaluating full spectrum envelopes for changes in "sister dye" absorbance peak intensities to assess degradation due to time, temperature, and exposure to tank metal, the complement of data was reduced to trends in absorbance intensity with time (number of days), shown in Fig. 27.

The comparison of this dye with the SiNaph does indicate acceptable performance, especially at nearfreezing and near-room temperature, but not in the long term at high $\left(120^{\circ} \mathrm{F}\right)$ temperatures. The sister dye's optical emission stability degrades on a timescale that precludes use in cases of retained evidence or extreme summer heat storage. This dye's stability would only be acceptable in the case of a duty cycle length of 1 business week or less. Although this may be adequate for the ultimate fielded protocol to monitor trucks in transit and at depots, it will not suffice for longer-range applications. Based on the results of these tests, the Fuel Marker team chose SiNaph to be utilized in the POC testing.

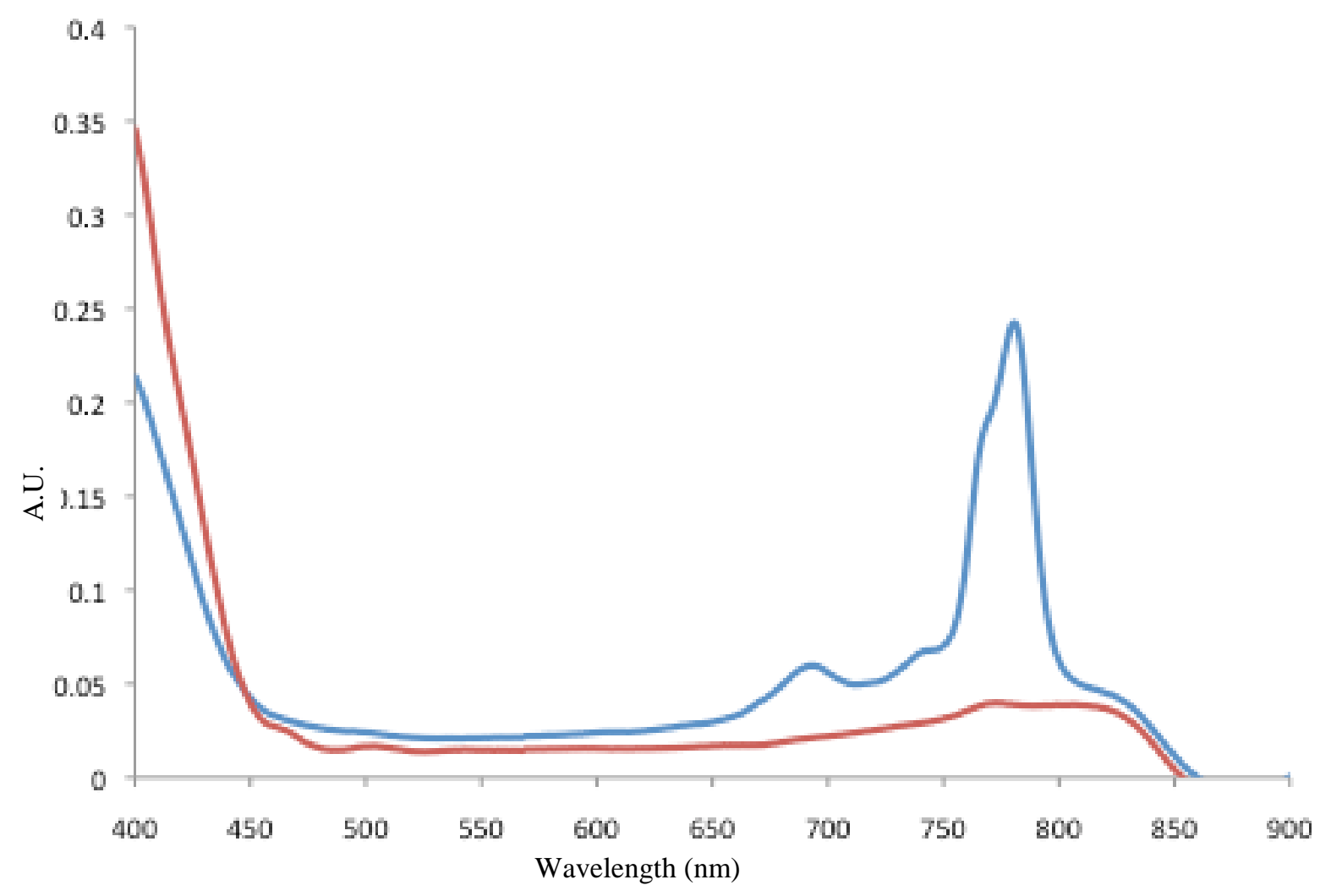

Fig. 26. Blue trace (with peak at $\sim 770 \mathrm{~nm}$ ) shows initial absorbance response of "sister" dye in diesel. Red trace is same sample’s absorbance after 28 days under exposure to tank metal at $120^{\circ} \mathrm{F}$. 


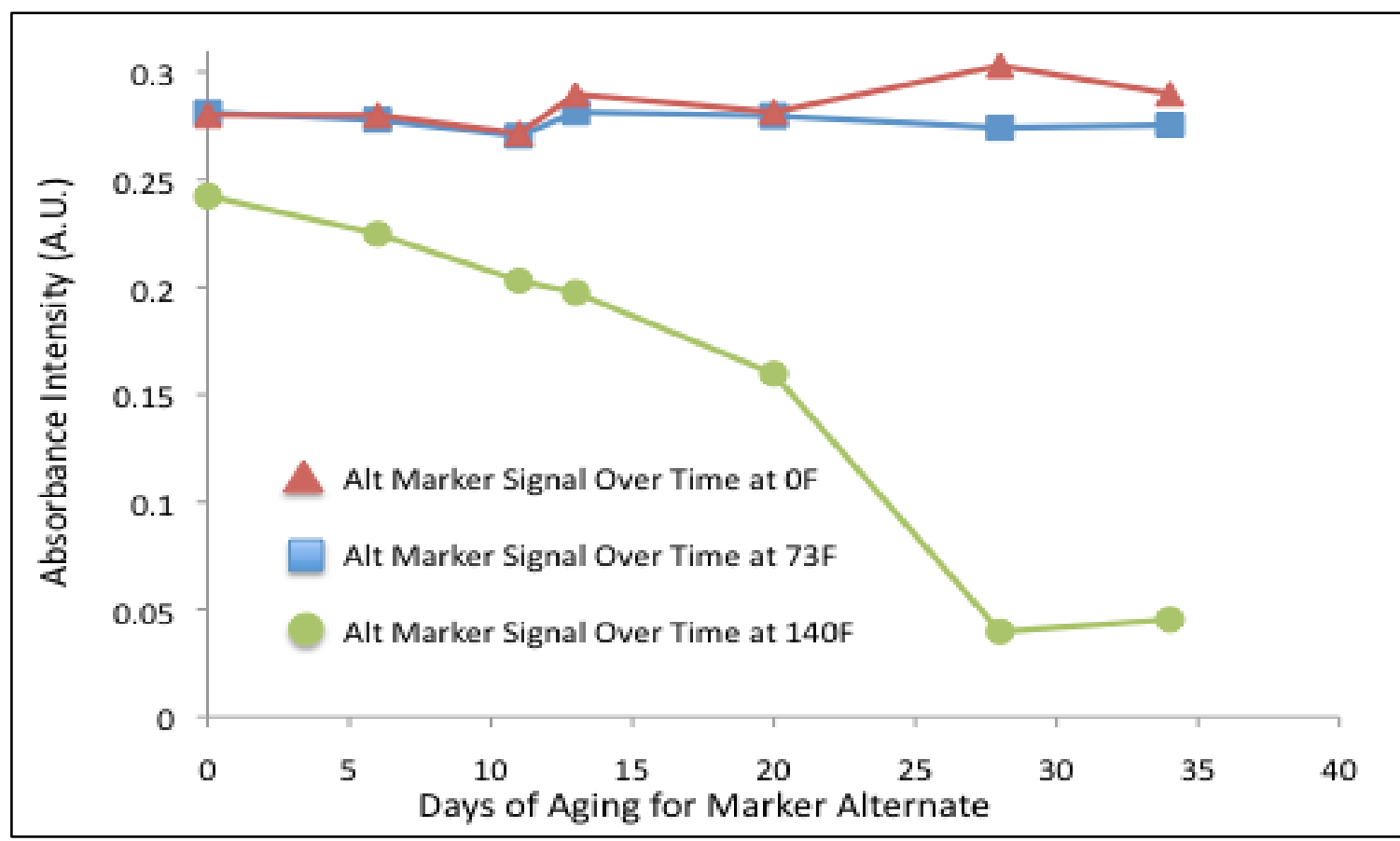

Fig. 27. Degradation of sister dye at low, room, and high temperature in presence of tank metal. 



\section{SENSORS}

A suite of sensors attached to a fuel transport vehicle provides the critical information needed to evaluate whether or not FTE is or has occurred. These sensors, the motivation behind their use, and any relevant details regarding unique development or implementation issues are presented in this section.

\subsection{GENERAL SENSOR SUITE CONSIDERATIONS}

In this section we consider the general design and layout of a sensor suite suitable for detection of FTE. We begin by considering the general functionality required of the sensor system.

\subsubsection{Required Functionality}

In the most general terms, the sensor suite must furnish information capable of answering the following questions:

- Are any of the access points into the trailer open?

- How much fuel is present-what is the fuel level?

- Is the fuel in the truck the fuel on which excise taxes were paid at the load point?

In the rest of this section we develop a general layout and Concept of Operations for how a sensor suite capable of answering these questions may be deployed on a fuel transport.

\subsubsection{General Trailer Layout and Operation}

Figure 28 below shows a schematic of a typical fuel trailer layout. The trailers are normally divided into three to four compartments, with each compartment having its own hatch, emergency valve, and fill valve.

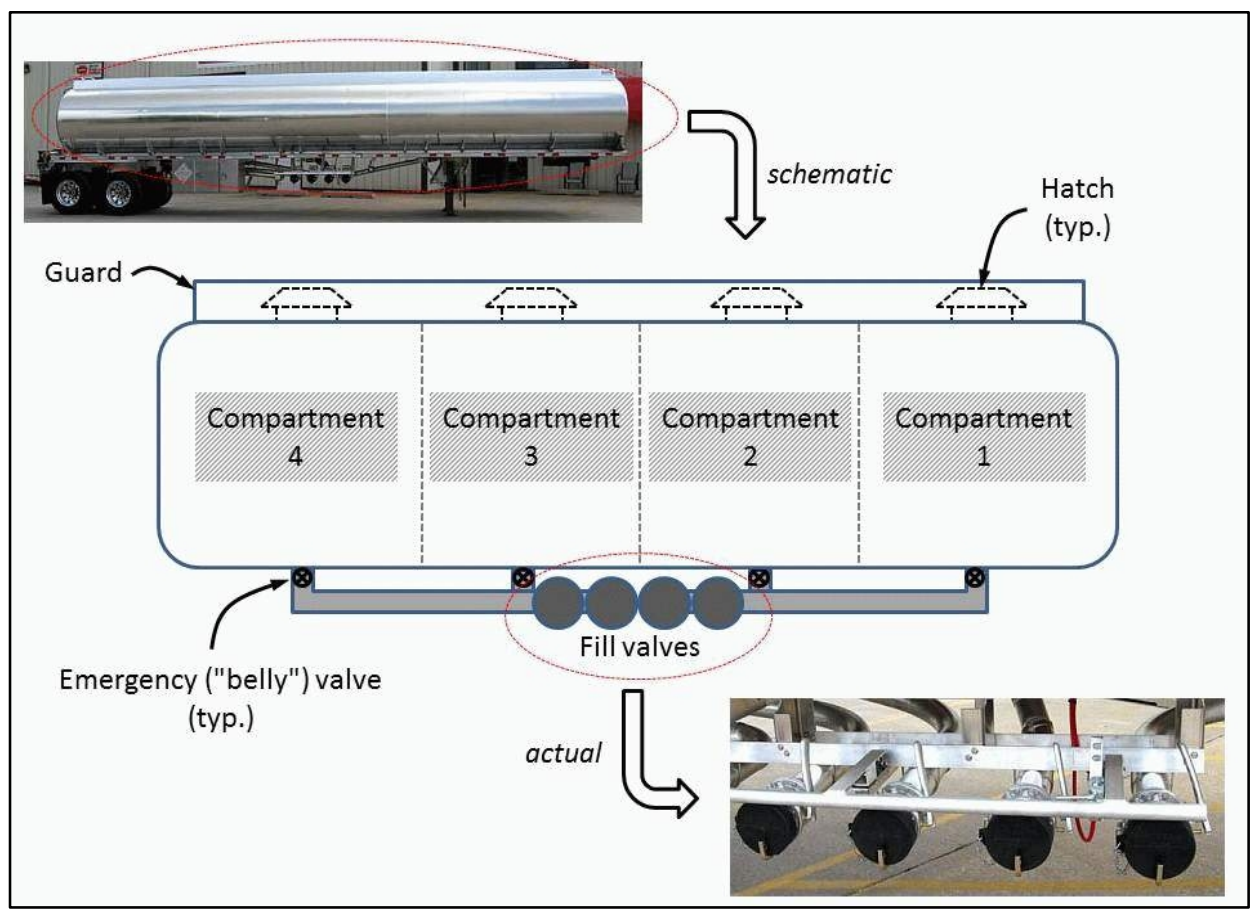

Fig. 28. Fuel trailer compartmentalization and access points. 
The emergency valve, by law, must be kept closed during over-the-road operation as it prevents uncontrolled release of the compartment contents should the plumbing and valves below it be compromised. Filling and emptying of the trailer is done through the fill valve. At the terminal rack, during the filling operation, the fuel is pumped in at a volumetric flow of about 500 gal per minute (gpm), while unloading (typically at a retail station) is done under gravity feed, so the flow rate will vary continuously throughout the off-load operation.

It is clear from Fig. 28 that there are two access points into each compartment, the fill valve and the hatch. It is also clear that each compartment will have its own fuel level. Therefore, in order to answer the first two of the three questions, we must monitor the hatch and valve on each compartment as well as the fuel level in each compartment. As for the third question, this requires the development of a "marker," the correct concentration of which indicates unadulterated taxed fuel, as well as a sensing system for measuring same. A detailed description of the marker selection methodology and characteristics of the selected marker are described in Sect. 2. For the purposes of this general discussion, we are considering only the general functionality of the sensing device.

\subsubsection{General Sensor System Specifications and Constraints on Installation}

The considerations above are adequate for laying out the general specifications for the individual sensors required. The sensors required are hatch and valve sensors, a level sensor, and a sensor to determine the marker concentration in the fuel. The general requirements on each of these sensors are as follows.

- Hatch and valve sensors:

- Binary output (open/closed) and

- Low or zero power consumption

- Level sensor:

- Range empty to full, resolution $\pm 200-300$ gal,

- Temperature, slosh and tilt effects compensated or minimized, and

- Accommodates presence of bottom valve.

- Marker sensor:

- Concentration range $0-1000 \mathrm{ppb}$, resolution $\pm 50 \mathrm{ppb}$,

- Temperature and flow effects compensated or minimized,

- Inline, real-time sampling strongly preferred, and

- Calibration (if required) infrequent.

In addition to these requirements, the addition of sensors to the trailer should not add measurably to the height or width of the trailer and should not affect the ground clearance of the trailer. The sensors should also ultimately be able to satisfy intrinsic safety requirements necessary for operation at the terminal rack and other hazardous locations, although this requirement could be waived for the field tests to be conducted in a very controlled test environment. Finally, since a loaned trailer from Pilot was to be used for the field test, installation of the sensor suite could not require any penetration of the fuel trailer or permanently modify the combination vehicle in any way.

In the following sections, the detailed considerations that went into the selection and development of the various sensors are described. The hatch, valve, level, and marker sensors are considered. Finally, in Sect. 3.5, we describe the hardware developed to mount the sensors onto a fuel transport trailer for the field test. 


\subsection{HATCH AND VALVE SENSOR DEVELOPMENT AND EVALUATION}

\subsubsection{Approach and Sensor Selection and Off-Vehicle Evaluation}

Detection of hatch or valve opening and closing is an exercise in position sensing. Since modern industrial automation has created significant demand for position-sensing technologies, there was high confidence that commercial, off-the-shelf (COTS) technologies could be identified to meet this need. Very rapidly the search for appropriate position-sensing technologies converged to the "GO" switch technology offered by TopWorx (Louisville, Kentucky). The GO switch is an explosion-proof, magnetic proximity switch developed for use in hazardous locations. The switch will close when a magnetic target is moved to within a certain distance (normally 1/4 in.) of the sensing face. A sample installation (image courtesy of TopWorx) is shown in Fig. 29.

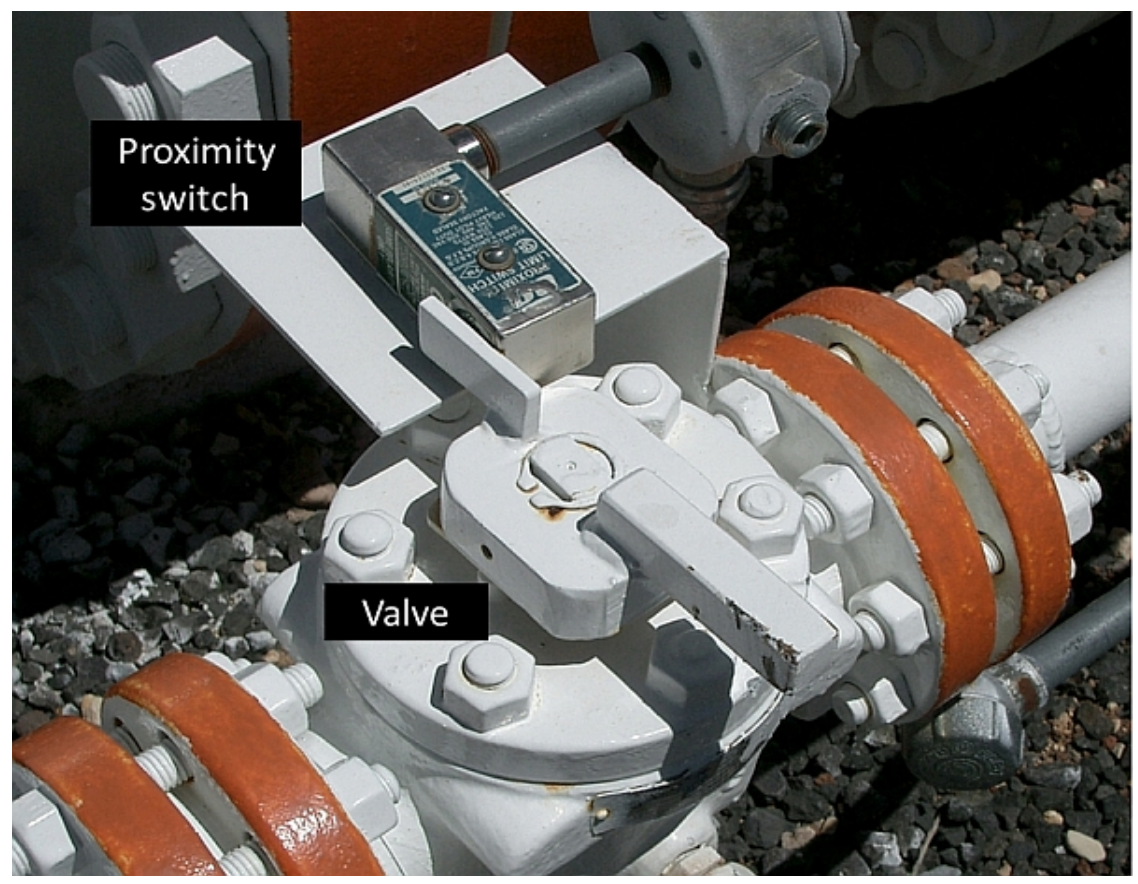

Fig. 29. Sample GO switch installation to monitor valve position.

Using a similar arrangement to that shown in Fig. 29, GO switches can be connected to the opening mechanisms on fuel valves and fuel compartment hatches to detect when the valves/hatches are open or closed.

\subsubsection{Off-Trailer Evaluation}

Figure 30 shows the configuration used to test the functionality of the TopWorx hatch and valve sensors. The switching action occurs between the wires shown connecting to the side of the proximity switch in Fig. 30 and was readily verified for the TopWorx switches. Therefore these sensors were deemed suitable for use in the field test. 


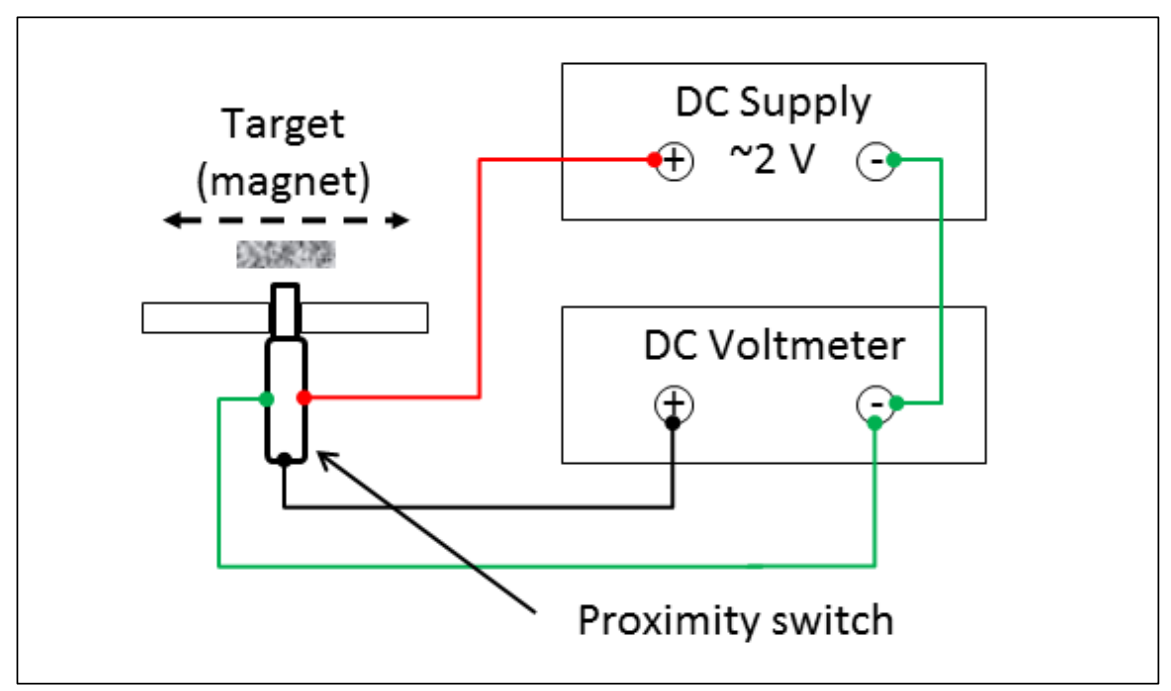

Fig. 30. Setup for off-trailer evaluation of hatch and valve sensors.

\subsection{LEVEL SENSOR DEVELOPMENT AND EVALUATION}

\subsubsection{Approach}

As was the case with the sensing of valve and hatch position, level measurement of fluids is a common process engineering challenge. There are several general approaches that can be used, as described in the summary by Boyes. ${ }^{2}$ There have also been specialized techniques developed just for hydrocarbons. We will review both of these briefly here. According to (Boyes, 2007), the primary general techniques that may be applicable to hydrocarbons are ultrasonic, radar, and differential pressure measurements. Ultrasonic measurements function by "time of flight," whereby an ultrasonic pulse is launched either from the container bottom (nonintrusive) or from above the liquid (intrusive). This wave will reflect at the interface between the liquid and the gas due to the large mismatch in "acoustic impedance." The reflected wave is detected by a suitable transducer, and then the level can be calculated from the velocity of sound in either the air or the liquid, depending on whether the operation is intrusive or nonintrusive. Radar measurements are similar to ultrasonic but radar instead of ultrasonic waves is used, and nonintrusive techniques are not possible.

Differential pressure level measurements function as shown in Fig. 31. A differential sensor is required because $p_{\text {vapor }}$ (the vapor pressure in the tank above the surface of the fuel) will vary with temperature. In our specific application, $\mathrm{p}_{\text {vapor }}$ will vary between the limits set by a DOT-mandated relief valve (not shown in Fig. 31) that relieves at 1 psi for pressure and 3/8 psi for vacuum. ${ }^{3}$

Several vendors offer versions of the above technologies that are safe to use with hydrocarbons - they are intrinsically safe or explosion proof. For example, guided wave radar sensors for petroleum product level measurements are offered by Titan Pacific Resources. ${ }^{4}$ There are also novel mechanical measurement technologies developed just for hydrocarbons. JOWA Consilium manufactures a system with the trademark name "Petrotape." In this system a tape containing two spatially separate conductors is suspended in a perforated stillpipe inside the tank. Below the fluid level, the hydrostatic pressure of the fluid will press the two conductors together, lowering the electrical resistance of the immersed length and enabling an inference of the fluid level to be made. Other similar devices from other manufacturers use capacitance. 


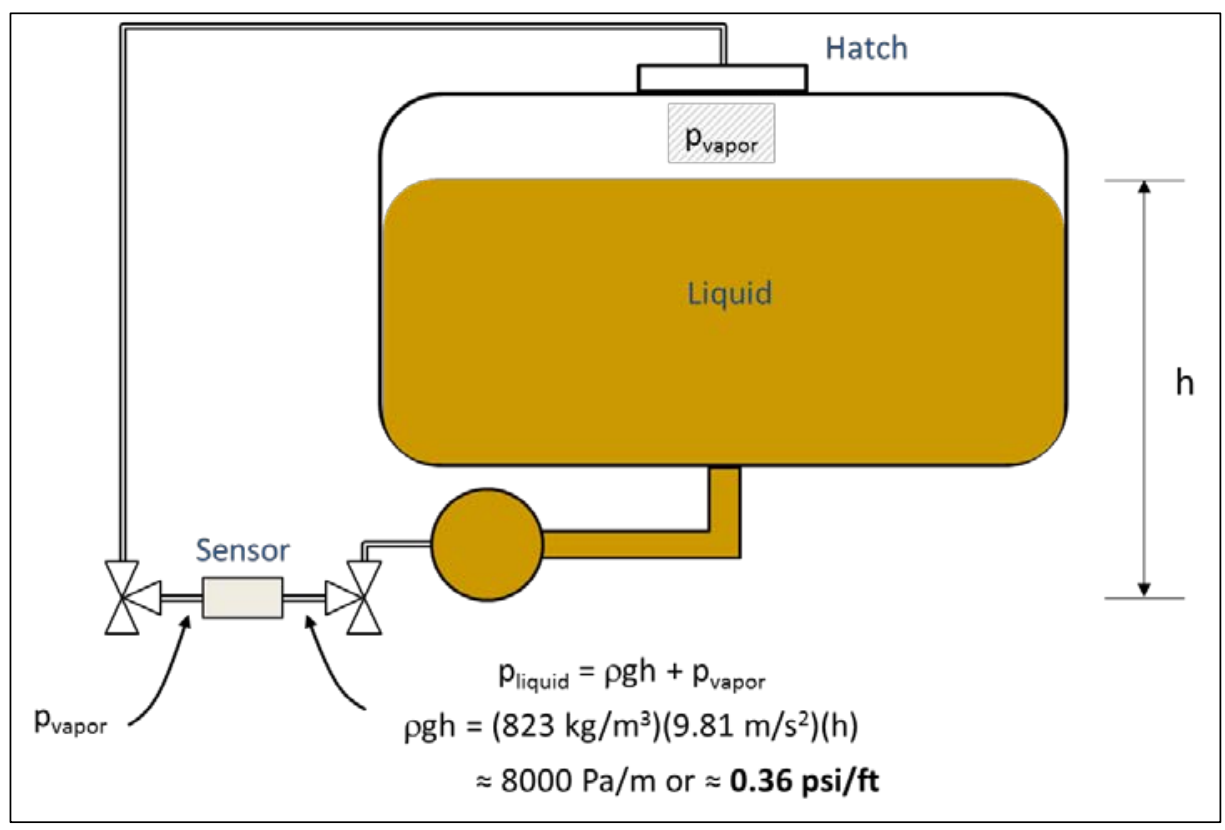

Fig. 31. Differential pressure sensor schematic of operation.

It is clear from the foregoing information that there are many options for level measurement; however, the constraints imposed by the inability to penetrate the trailer, and the need to operate over the road, eliminate many of these sensors. The inability to penetrate the trailer eliminates the guided wave radar, intrusive ultrasonic, and Petrotape-style options, as these are typically bulky and would exceed the height of the guard in Fig. 28. This leaves only nonintrusive ultrasonic and differential pressure, with penetration for the $\mathrm{p}_{\text {vapor }}$ measurement (Fig. 31) being made on a replacement hatch specifically purchased for the field test.

Prior work at ORNL had indicated one challenge in ultrasonic distance (level) measurement was the need for the interface being detected to be parallel with the transducer face. Relative tilts between the transducer face and the interface of even a few degrees drastically reduce the amplitude of the detected reflection. Since in over-the-road applications there will be significant tilt and slosh of the liquid, sophisticated correction algorithms ${ }^{*}$ need to be applied. Such challenges in fact led to the discontinuation of a commercial nonintrusive ultrasonic level sensing system for petroleum tankers that was offered by Canongate Technology (United Kingdom). Therefore we decided to adopt pressure-based level sensing techniques for the field testing. Furthermore, given that the testing was a POC only, it was decided to employ simply absolute-pressure-based technology for the level measurement.

Intrinsically safe pressure transducers are available from a wide number of vendors, and the selection was made based on (1) the desired pressure range and (2) the desire by our third-party partner (AirWeigh) to have a ratiometric output transducer with MetriPack connections. The desired pressure range is easy to deduce from the relation given in Fig. 31, $\mathrm{p}=\rho \mathrm{gh}$, where $\rho$ is the density of diesel fuel $\left(\sim 830 \mathrm{~kg} / \mathrm{m}^{3}\right)$, $g$ is the acceleration due to gravity $\left(9.8 \mathrm{~m} / \mathrm{s}^{2}\right)$, and $h$ is the height of the fuel. Therefore a column of diesel will generate pressure on the order of $0.36 \mathrm{psi} / \mathrm{ft}$, indicating that a pressure sensing range of $0-5 \mathrm{psi}$ (corresponding to 0-14 ft) would be nearly ideal. An intrinsically safe sensor with this measuring range as well as the electrical features (ratiometric output, Metripack connector) requested by AirWeigh was

*See correction algorithms such as those found in “Accurate Fluid Level Measurement in Dynamic Environment Using Ultrasonic Sensor and v-SVM.” Terzic, Jenny, Nagarajah, Romesh and Alamgir, Muhammad. 10, October 2009, Sensors \& Transducers, Vol. 109, Issue 10, October 2009, pp. 76-91. 
available from American Sensor Technologies and purchased for the field testing. A picture of the sensor is shown in Fig. 32.

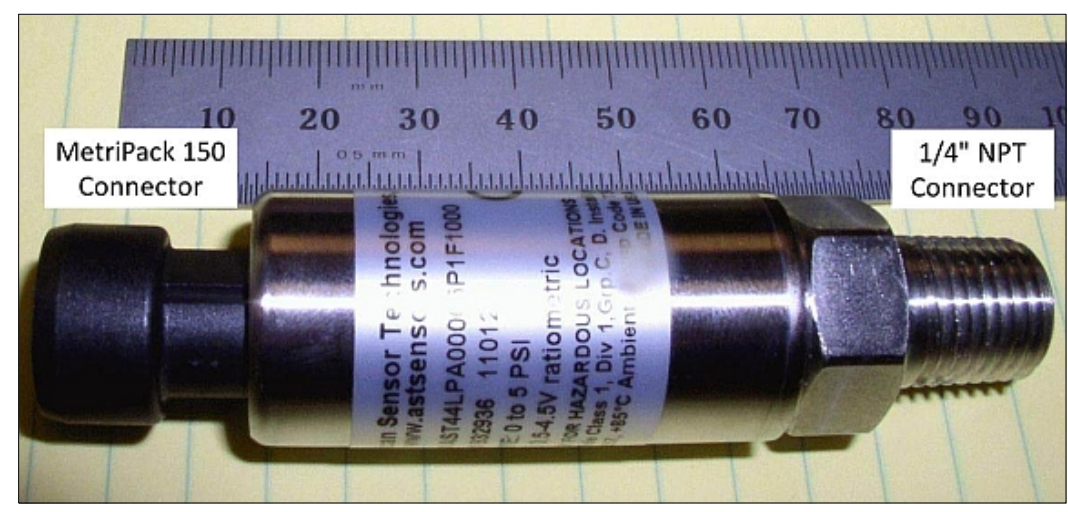

Fig. 32. Pressure-based level sensor used for POC testing.

\subsubsection{Sensor Selection and Off-Vehicle Evaluation}

Using a benchtop rig that had been assembled prior to the field testing, the operation of the level sensor could be investigated off-trailer. The setup for this is illustrated in Fig. 33(a), and typical results are shown in Fig. 33(b) and (c). The output from the sensor was linear with fluid height over the range that was available for investigation, and the ratiometric output was verified for supply voltages between 5 and 6 voltage direct current (VDC). Therefore this sensor was deemed suitable for use in the field testing.

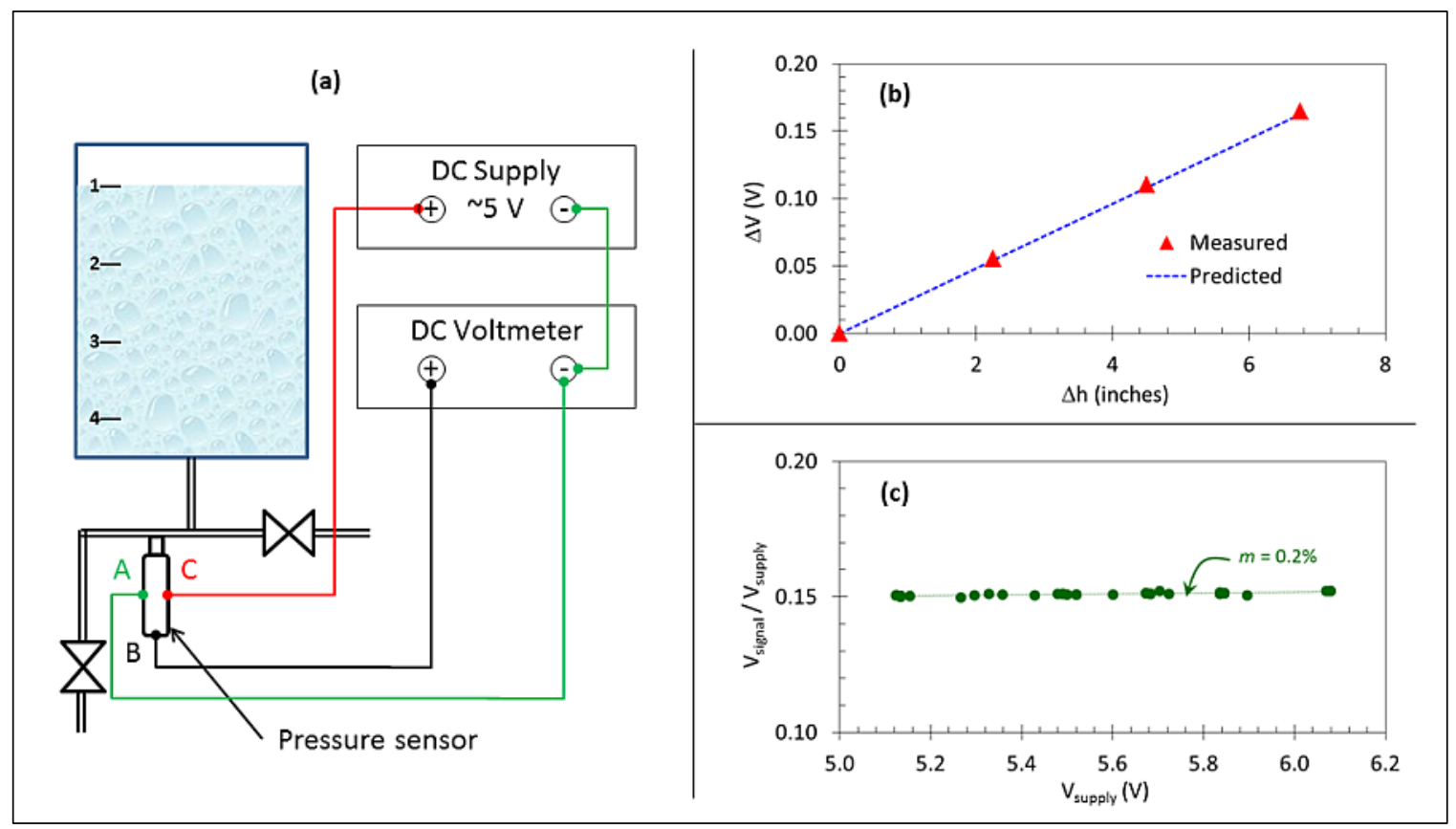

Fig. 33. Setup for off-trailer evaluation of level sensor and results. 


\subsection{MARKER SENSOR DEVELOPMENT}

In contrast to the hatch, valve, and level sensors, the marker sensor required developing a sensor and sensing technique that had no commercial counterpart. Although accurate off-line measurements of fuel marker concentrations are possible in the laboratory, no method of on-vehicle monitoring was available at the start of this project. On-vehicle measurements are challenging because accurate absorption and fluorescence measurements - the primary method of measuring fuel marker concentration —are extremely difficult to make without a highly controlled reference sample to compare against. Reference samples are easily established and utilized in a laboratory setting, but for a vehicle in the field — where diesel fuel is being loaded and unloaded from various sources-reference samples are not a feasible requirement. As such, a self-referencing method of measuring diesel fuel marker concentration was desired and needed to be developed. This section describes the development efforts undertaken to achieve this new type of sensor.

\subsubsection{Approach}

When illuminated with ultraviolet (UV) light, diesel fluoresces and produces blue light. An additive that fluoresces at a different color can be added to the fuel to serve as a fuel marker. The higher the marker's concentration in the fuel, the larger the non-blue fluorescence will be relative to the diesel's natural blue fluorescence. This is the basic measurement technique behind fuel marking.

To enhance this basic sensing technique, ORNL developed a custom fuel marker that fluoresces in the red to near-infrared (NIR) region of the optical spectrum (Sect. 2). This marker was unique in that it only weakly fluoresced when illuminated with ultraviolet light but strongly fluoresced when excited by red light. By separately exciting and measuring the fluorescence of marked diesel with both UV light and red light, the amount of diesel fuel present versus the amount of marker present can be accurately determined. This measurement produces relative marker concentrations that are expressed in parts per billion (ppb).

Most importantly, this technique is "self-referencing," meaning that it can determine a relative marker concentration value without the need for an external reference sample or external calibration source. Given the various types and sources of diesel fuel that must be monitored for FTE, a "self-referencing" method is critical to real-world implementations. The research and development steps required to design and produce this sensor are detailed below.

\subsubsection{Optical Design}

A proof-of-principle apparatus was first constructed on a large optical breadboard (Fig. 34). It was tested to verify feasibility and estimate achievable resolution. Laboratory tests demonstrated that the basic approach was sound and could likely detect marker concentration changes in the tens of ppb range. The technique's exact implementation, however, would be highly dependent upon the details of the marker still under development and, as such, any prototype design needed to maintain flexibility in its design to permit future modification and changes to the marker specification/performance. In addition, the system needed to be low cost, low power, and rugged. A cost of $\$ 100$ (for a production unit) was targeted with an environmental rating, ultimately, of IP65.

Once the proof-of-principle experiment had been demonstrated, the basic optical components utilized in the experiment were re-specified for use in a compact, prototype sensor. These components and the basic optical system were modeled using ZEMAX ray tracing software and are shown in Fig. 35.

As can be seen in Fig. 35, the optical outputs from a red and blue/UV light-emitting diode (LED) are combined with the help of a glass focusing lens. This combined output is imaged through a glass "cover 


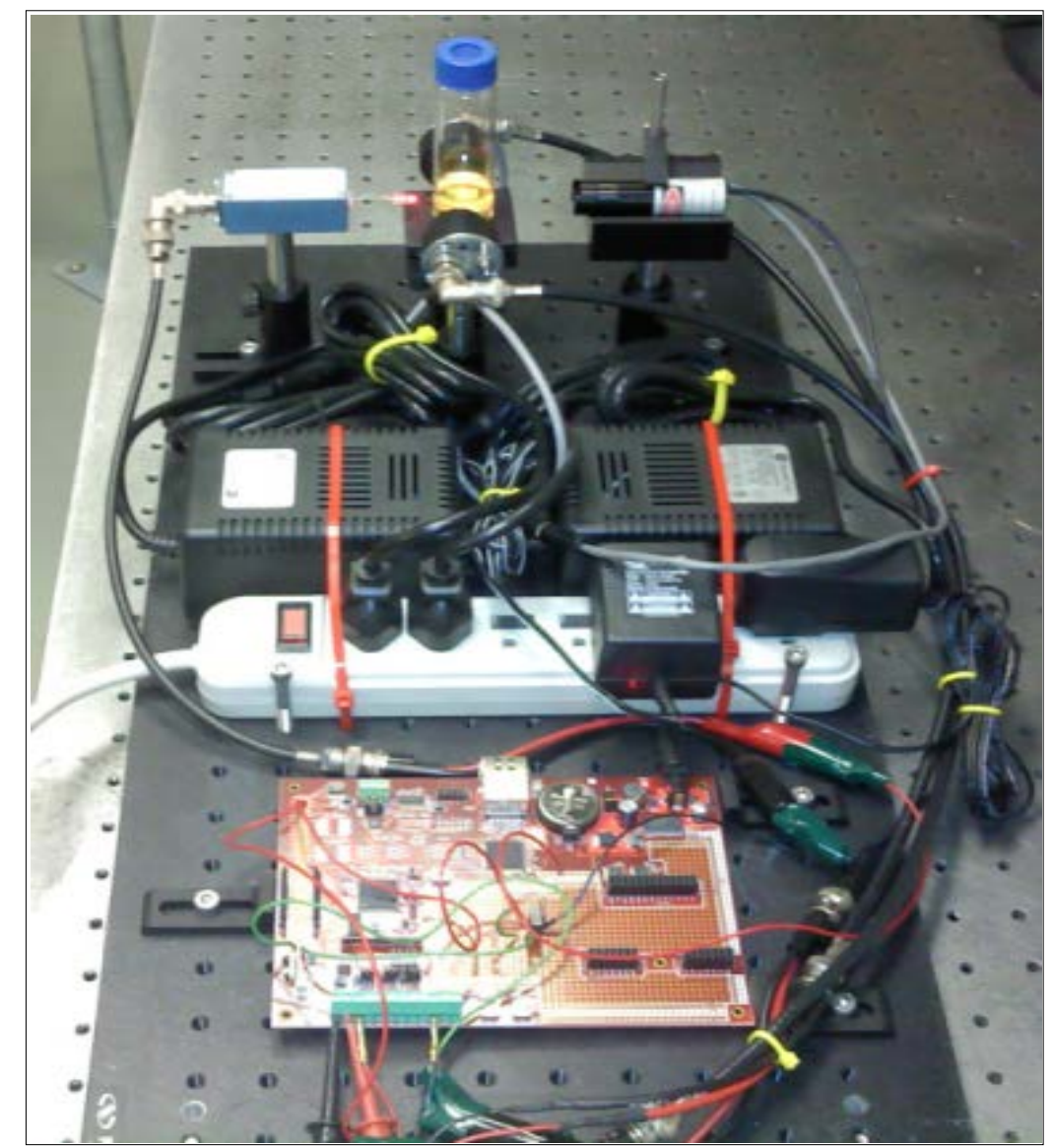

Fig. 34. Proof-of-principle fuel marker sensor breadboard experiment.

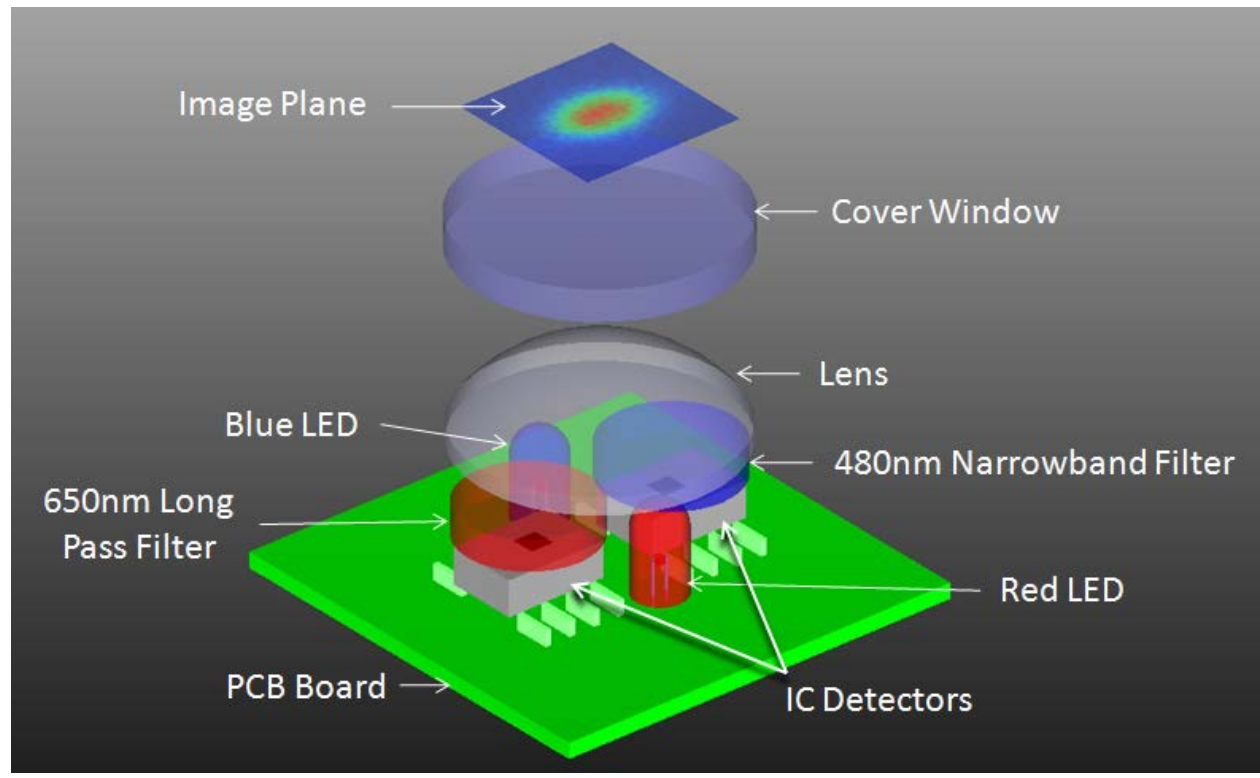

Fig. 35. Optical model of prototype fuel marker sensor. 
window" and onto an imaging plane roughly 1 in. from the LEDs. Under normal operations, diesel gas is located in the image plane (beyond the cover window), producing a fluorescent signal that scatters light back through the cover window. This fluorescent light is then collected by the lens and imaged onto the two integrated circuit (IC) optical detectors attached to the printed circuit board (PCB) adjacent to the LEDs.

The IC detectors are silicon detectors that offer broadband sensitivity. However, an optical filter (either red or blue) is covering each of the IC detectors, making the detector only sensitive to a defined band of the fluorescent spectrum. A plastic optical baffle structure is located between the two LEDs and two detectors. The baffle structure reduces the amount of internal reflection and direct illumination of the detectors by the LEDs.

This prototype device allows two different wavelength LEDs to excite the same region of a fuel sample (at separate times) while using two separate detectors to measure different corresponding regions of the fluorescent spectrum. The sensor's optical design has been optimized to ensure a high degree of isolation between the two measurements. This is critical to detecting small changes in concentration. The spectral sensitivity of the filtered detectors, as well as the fluorescent response of marked diesel (at two different excitation bands), is shown graphically in Fig. 36. The blue region corresponds to the bandwidth of the blue filter, while the pink region corresponds to the bandwidth of the red filter.

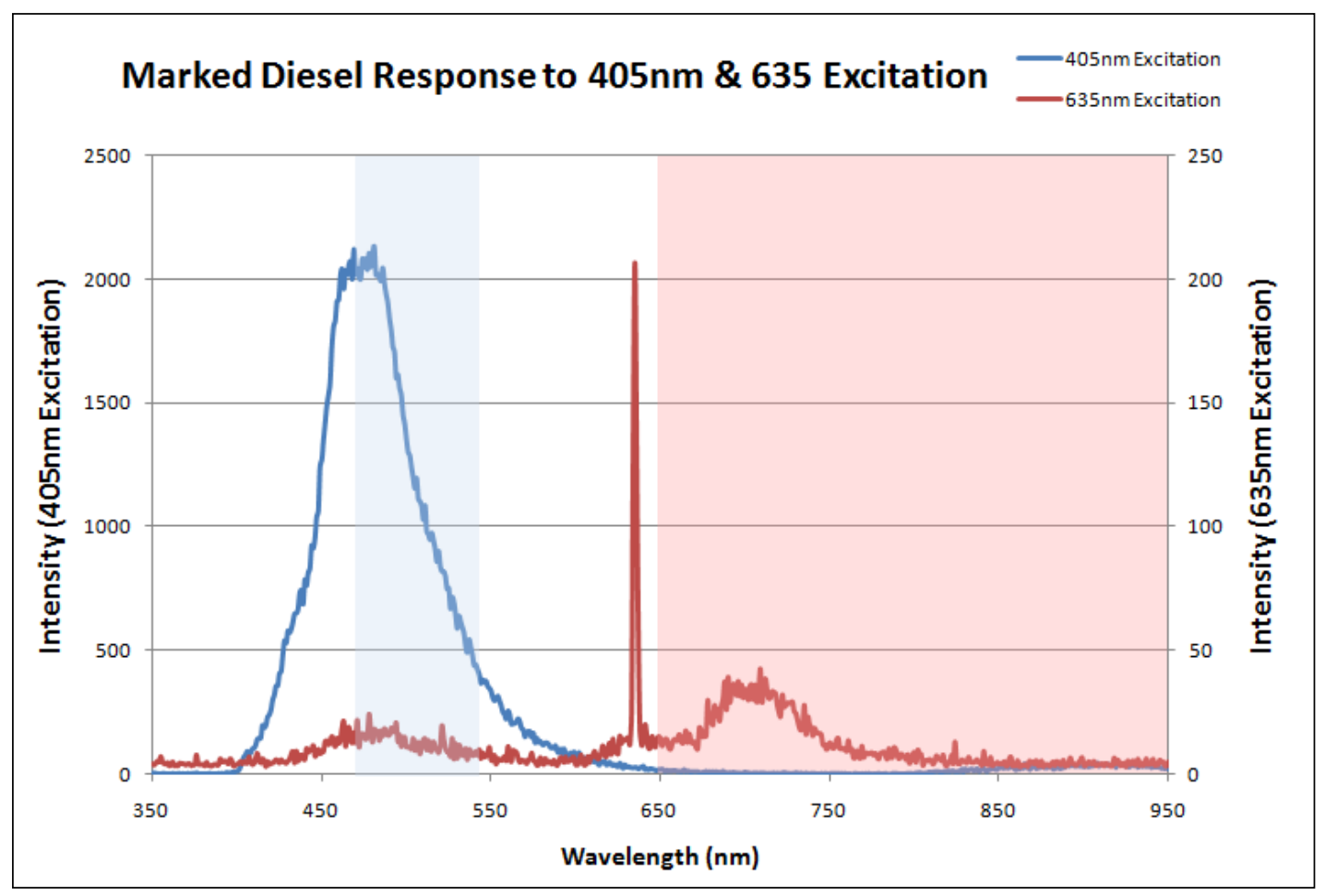

Fig. 36. Spectral response of marked diesel at two excitation wavelengths.

As mentioned earlier, the "blue” peak in Fig. 36 is a natural by-product of diesel (marked or unmarked), while the "red" peak is a by-product of the custom marker. Measuring and dividing the intensity of the two peaks gives a "self-referenced" measurement of the marker concentration strength.

To physically realize the design in Fig. 36, two identical detectors were used to detect the amount of red and blue light fluorescing from marked diesel. To ease assembly and reduce size, a photodetector with an integrated amplifier was used in a surface mount IC package. It offered broadband spectral response, high 
sensitivity (0.4 A/W), suitable speed (14 kilohertz), low cost $(\approx \$)$, and low quiescent power consumption $(\approx 1.5 \mathrm{~mW})$.

Two LEDs, one red and one blue, were used to separately excite the diesel fuel. To improve signal quality and meet power requirements, these LEDs were required to produce a large flux of light into a narrow beam with minimal power consumption. Some of the best LEDs for this purpose are manufactured by Roithner LaserTechnik (a German company) and were utilized in this prototype design. These LEDs have a center wavelength (405 nm and $630 \mathrm{~nm}$ respectively) that is optimal for excitation of either the diesel or the selected fuel marker.

As mentioned, two optical filters (one red and one blue) were used to isolate portions of the fluorescence spectrum during excitation by one of the LEDs. These filters were required to offer good extinction of the excitation bands while providing good transmission of the nearby spectral region-of-interest. There are many high-quality dielectric stack filters that provide exceptional spectral control for exactly this type of application. Unfortunately, though, most of these filters were too expensive for our target cost. To balance cost and performance, two filters were chosen that minimized cost while still providing good extinction of the excitation bands. Where possible, colored glass filters were used instead of dielectric stacks to reduce cost. The blue filter used was a narrow band interference filter that was centered on $480 \mathrm{~nm}$. The filter was more expensive $(\approx \$ 4)$ than a colored glass alternative, but it provided a narrow transmission bandwidth that was more likely to capture just diesel fluorescence and not other unwanted commercial markers. The red filter used was a low-cost colored glass filter.

The output from the LEDs needed to be concentrated into a single, centralized region. Otherwise, the red LED and blue LED would measure different portions of the sample, and more importantly, the detectors would not be able to optimally collect the refocused fluorescent signal. A simple concave lens (30 mm focal length) allowed us to achieve this with acceptable efficiency. The lens has a magnesium fluoride anti-reflection coating on all surfaces to minimize the possibility of back reflections into the detectors (which would increase the noise of the detectors).

By design, the lens focuses the LED light with a high degree of spherical aberration. This created a large region where the focal width remained largely unchanged. This region, called the "region of least confusion," is the optimal location for stimulating fluorescence. This region is approximately $6 \mathrm{~mm}$ deep by $5 \mathrm{~mm}$ in diameter.

To create a design that can be sealed from the environment, the "region of least confusion" was located just beyond a glass window. The design, therefore, assumes that the diesel fuel to be examined will be present 3 to $9 \mathrm{~mm}$ beyond this glass window. The window is $25 \mathrm{~mm}$ in diameter and coated with a magnesium fluoride anti-reflection coating to guard against stray reflections into the detector. It was assumed that this window would be the last optical surface in the sensor and would integrate with a gasket in the sensor housing to form a watertight seal.

All of the optical components described were modeled in ZEMAX Raytracing software to evaluate system performance and tolerance requirements. The model was then optimized to maximize the number of photons collected at the two IC detectors while simultaneously minimizing alignment requirements.

The greatest challenge of this design was to efficiently "centralize" the optical outputs of the two LEDs. Because both LEDs have a fair amount of divergence (which is different for each LED), optimization of the focusing lens' position was a primary concern. Figure 37 and Fig. 38 show the location in the focal plane of each LEDs focal spot. 


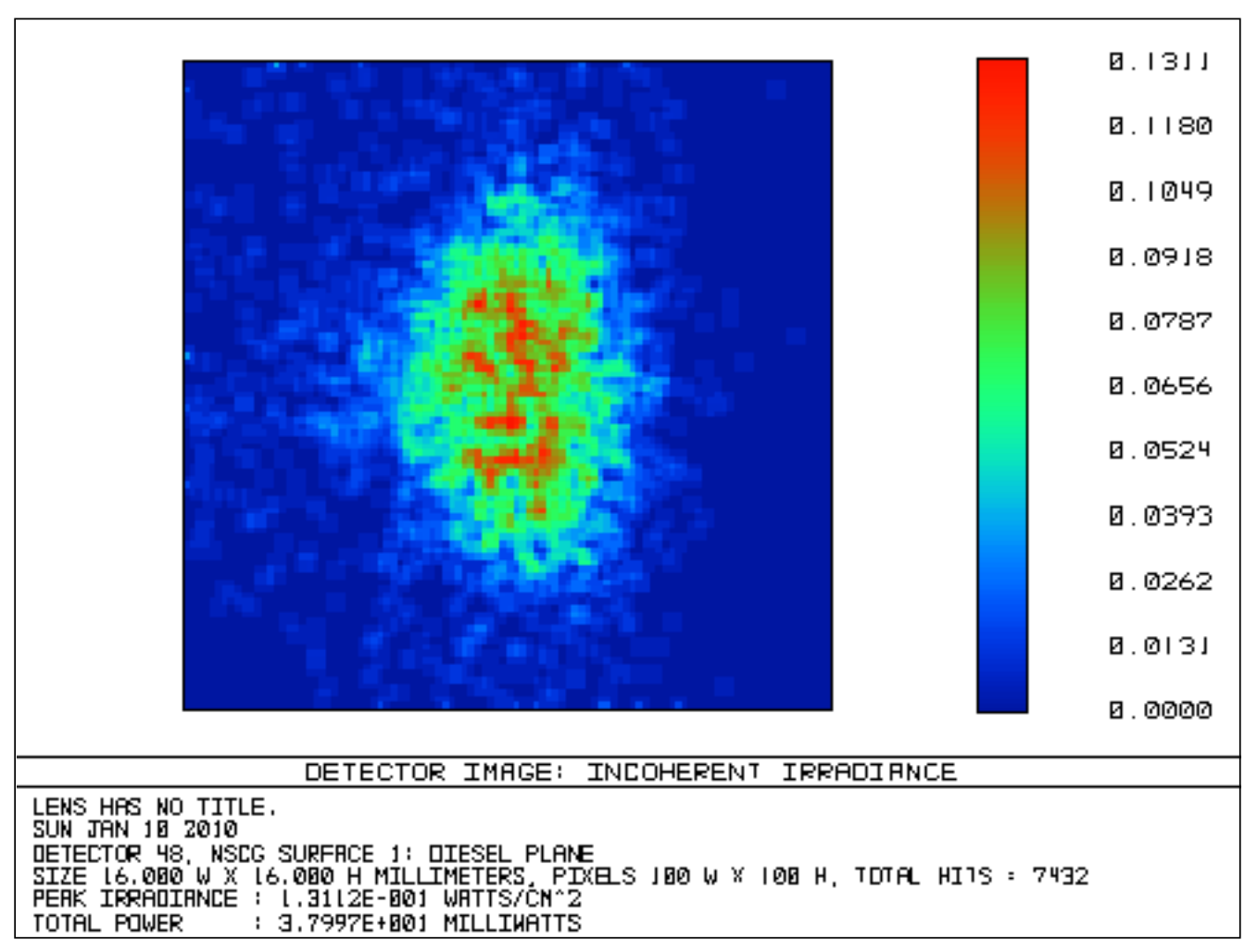

Fig. 37. Focal point for blue LED (at $6 \mathrm{~mm}$ beyond cover window).

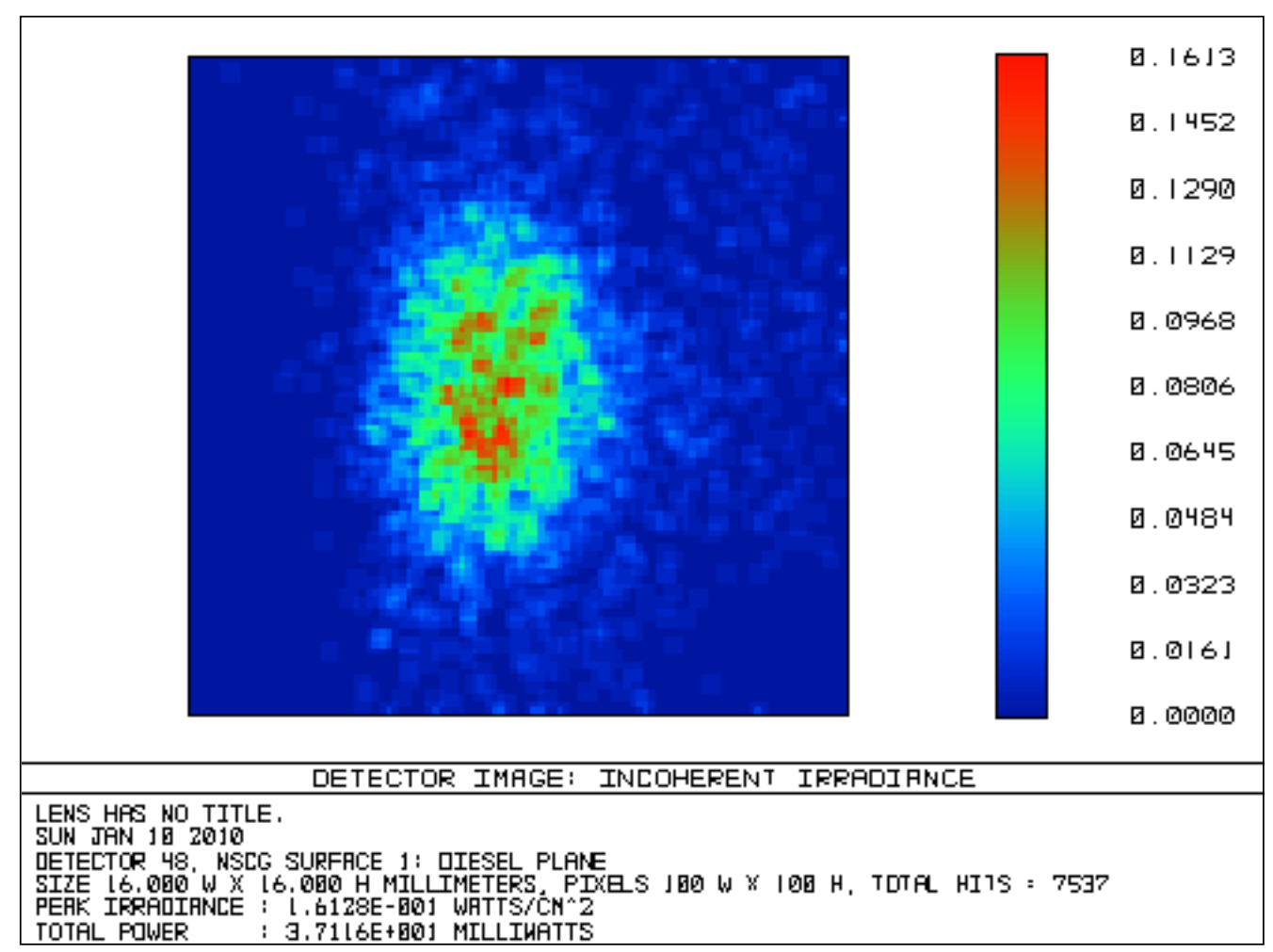

Fig. 38. Focal point for red LED (located $6 \mathrm{~mm}$ beyond cover window). 
As can be seen in Fig. 37 and Fig. 38, there is a slight displacement between the focal regions of the two LEDs. However, this configuration produced the largest modeled return signal for the two IC detectors. The optical efficiency of the simulated design at focusing and collecting light into a centralized region was approximately 38\% - with the majority of losses coming from internal reflections and non-focused light (due to the high divergence of the LEDs). For the LEDs selected, that represents approximately $4 \mathrm{~mW}$ of illumination for both the red and blue LEDs, which is comparable to (or greater than) the light energy levels used in early breadboard experiments.

As mentioned previously, the focused light experiences a high level of spherical aberration to create a $6 \mathrm{~mm}$ long region where the focal distribution is relatively unchanged. Figure 39 details the change in focal distribution from 3 to 9 mm beyond the system's "cover window."

As can be seen in Fig. 39, the change in focal distribution is small over the measurement window.

The ZEMAX model of the optical design was used to produce the optimal configuration and positioning of detectors, sources, and support optics. This information was critical to the design of the mechanical housing that followed, which would maintain the mechanical position and alignment tolerances necessary to ensure proper operation.

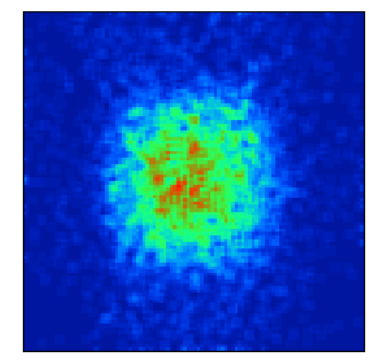

(a) $3 \mathrm{~mm}$

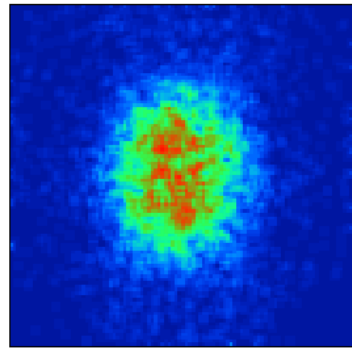

(b) $5 \mathrm{~mm}$

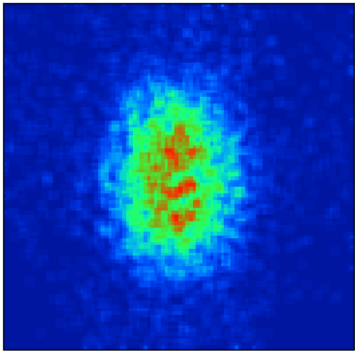

(c) $7 \mathrm{~mm}$

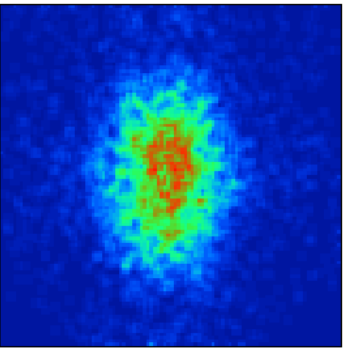

(d) $9 \mathrm{~mm}$

Fig. 39. Variation in focal distribution with distance from cover window.

\subsubsection{Mechanical Design}

The sensor's mechanical housing design was required to serve three main functions:

- Must accurately position optical components,

- Must provide environmental protection for electronic components (IP65), and

- Must provide a rugged mount for integration with a fuel tanker vehicle.

Simultaneously meeting all of these requirements in a small package and at minimal cost was a considerable challenge. To provide as much design flexibility as possible, a plastic housing was pursued that was fabricated using a stereolithographic 3D printing technique. The 3D printing technique allowed for rapid prototyping of sophisticated housing geometries and was well suited to the size and mechanical tolerance of this prototype sensor.

Initially, two housing designs were developed. One assumed an external power source, while the other incorporated an internal battery pack. Discussions with the Sensor Team concluded that it was unlikely that an internal battery source would actually be needed given the available DC power on the vehicle. Therefore, only the housing (without internal battery supply) is presented in this report. 
The sensor's prototype housing design is shown in an exploded view in Fig. 40.

The design is roughly the size of a deck of cards and incorporates all of the optical components specified previously. The optical components are carefully positioned relative to an internal electronics board, while peripheral electronics (i.e., switches, power receptacles, etc.) are panel mounted on the sides of the housing. Detailed descriptions of the mechanical dimensions and components are provided in Appendix B.

Four screws hold the front and back housing layers together, while a thin sheet gasket is compressed between the two halves to create a watertight seal. The front half of the housing also contains an optical window. Silicone is used to hold the window in place and to maintain the watertight seal. All external connectors are IP67 rated with an o-ring around the panel mount and are equipped with covers when not in use.

In addition to the housing's two main halves (front and back housing), an internal structure is also present that maintains the alignment of key optical components. The "Internal Optical Mount" is a plastic component that screws into four holes on the sensor's main PCB board. The mount houses a red and blue optical filter (held in place with epoxy) and positions them over the top of two board-mounted optical detectors. Red and blue LEDs are encapsulated by the mount to reduce the amount of stray light that might be incident upon the detectors. Finally, a 1 in. diameter lens is positioned at the front of the optical mount. The lens focuses the light from the LEDs toward an optical window located at the front of the sensor housing. Details regarding this mechanical component and the mechanical design in general are made in Appendix C.

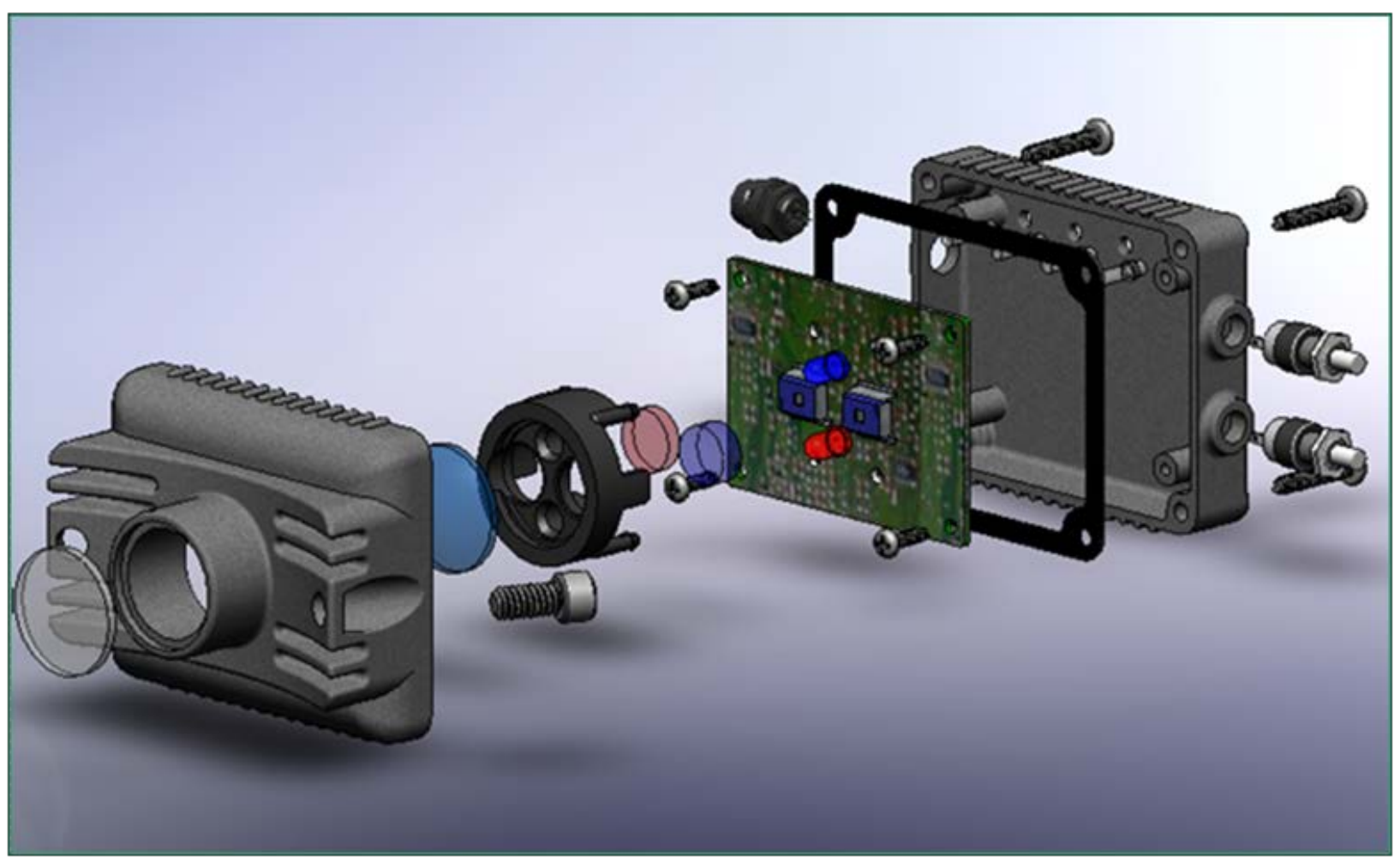

Fig. 40. Assembly view of sensor housing. 


\subsubsection{Electrical Design}

The sensor's electrical design was required to serve several functions, including the following:

- Must accurately position electro-optical components,

- Must pulse two LEDs and monitor two analog detectors,

- Must fit within the mechanical enclosure defined in the previous section,

- Must provide power regulation for use with 12 VDC vehicle power,

- Must provide 2 months of battery backup of internal operational parameters,

- Must have measurement speeds in excess of 10 samples/second,

- Must provide RS232/RS485 communications, and

- Must be individually addressable for use in networks.

Simultaneously meeting all of these requirements in a small package and at minimal cost was again a considerable challenge. To provide as much design flexibility as possible, a general-purpose microprocessor/microcontroller was selected as the controls platform. This processor

(Rabbit Semiconductor RCM3400) is an established processor that incorporates various analog input subsystems. The RCM3400 features a low-EMI Rabbit® 3000-based CPU subsystem running at 29.4 megahertz, with 512K Flash / 512K SRAM, 5 serial ports, and 8 channels of programmable gain analog input in an extremely small footprint $(1.37 \mathrm{in.} \times 1.16 \mathrm{in} . / 34 \times 29 \mathrm{~mm})$.

This processor was used to control all electro-optics hardware on a custom-designed PCB. Firmware was written in Dynamic C, and communications with outside devices were achieved over a 3-wire RS232 protocol.

The sensor's electrical design was broken into two sub-systems: power conditioning and electro-optical controls. Schematics of these two sub-system circuits are detailed in Appendix B. Fabricating a working electronics package required the development of custom PCB, integration of various connectors and peripheral components, and programming of the system's core processor. Based on the circuit design described in Appendix B, a four-layer PCB board was designed, routed, and fabricated. Where possible, surface-mount components were used to minimize the footprint of the components and maximize the use of both sides of the board. A complete Bill of Materials for these components and other ancillary components is provided in Appendix D.

\subsubsection{Firmware Development}

Appendix E provides the complete firmware source code for version 1.4 of the prototype sensor. This code was written in Dynamic C, a free compiler available from Rabbit Semiconductor and similar to most $\mathrm{C}+$ firmware environments. Appendix F provides an overview of the communication functions implemented by the firmware and made available to third-party developers who were partnering with ORNL to complete this project. These functions allowed the custom fuel marker sensor to communicate reliably with other third-party systems and sensors present in the field trial.

\subsubsection{Off-trailer Evaluation}

The completed prototype sensor is shown in Figs. 41 through 43.

To evaluate the sensors performance and sensitivity, eleven 40 milliliter ( $\mathrm{ml}$ ) vials of marked diesel were prepared at increasing levels of marker concentration ( 0 ppb, $100 \mathrm{ppb}, 200 \mathrm{ppb}$. . , $1000 \mathrm{ppb}$ ). The marker sensor was then used to evaluate each vial, in the laboratory, to discern the level of noise and sensitivity exhibited by the device. These tests showed that the sensor response was roughly linear and, given the noise levels exhibited, had a resolution of approximately 20 ppb (Fig. 44). 


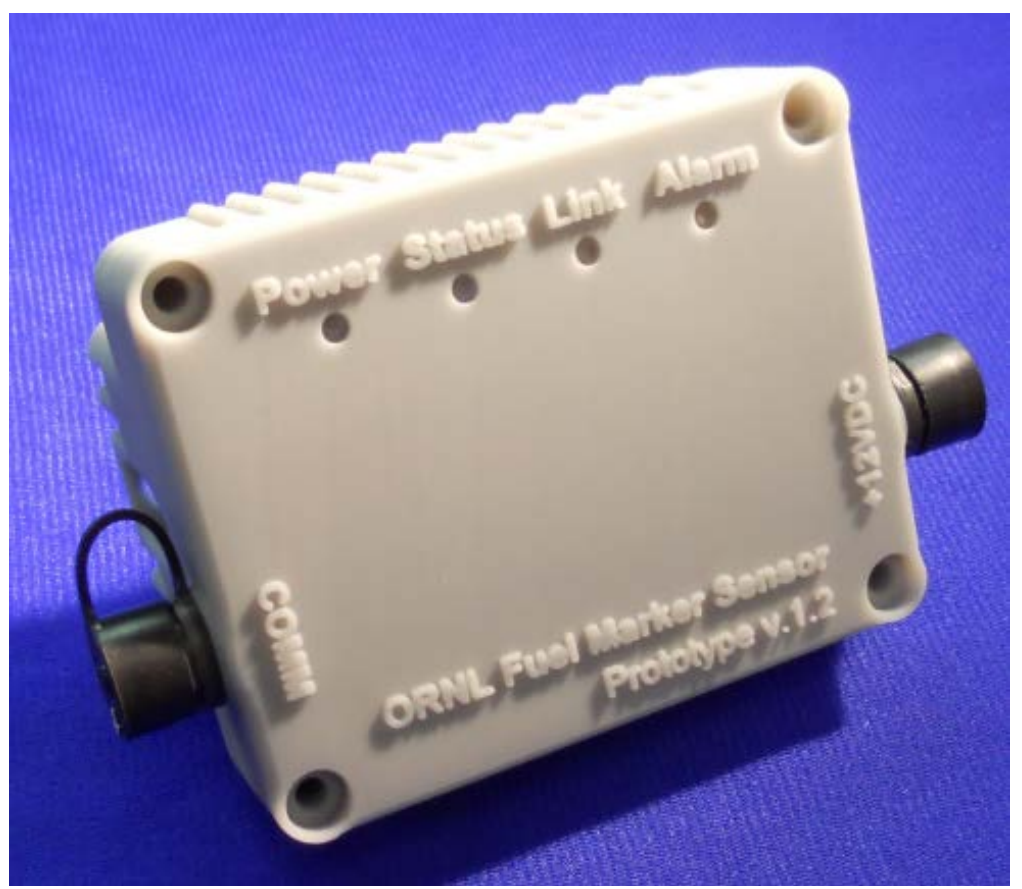

Fig. 41. Marker sensor back view.

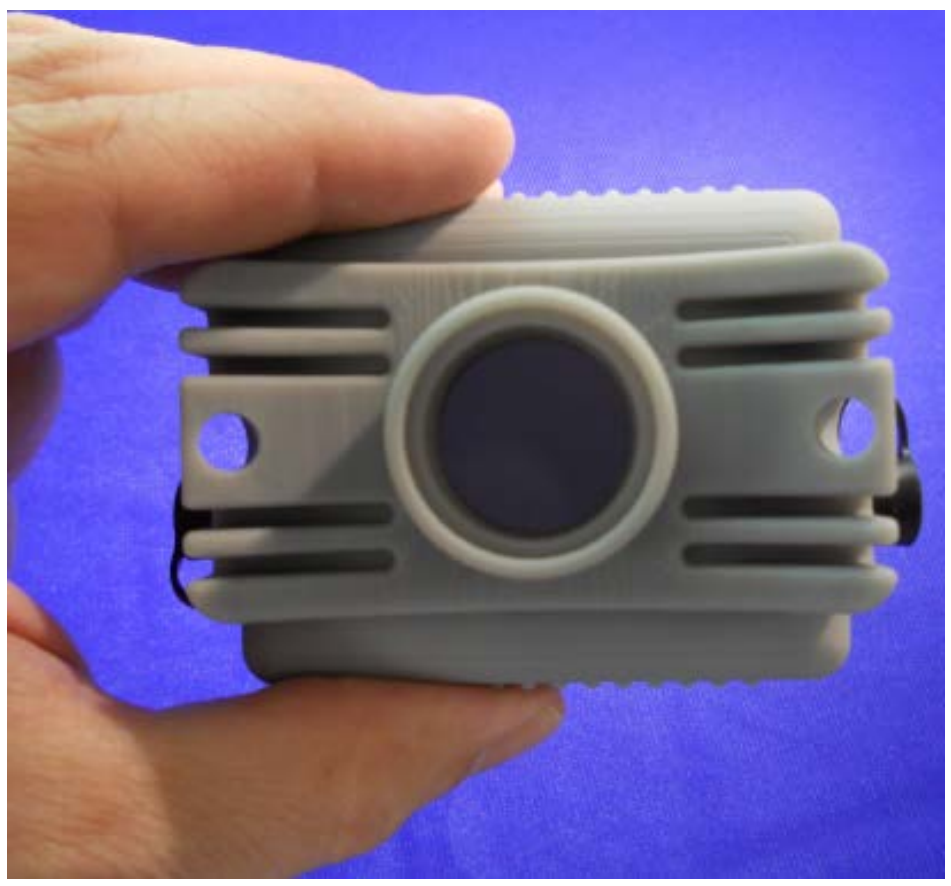

Fig. 42. Marker sensor front view. 


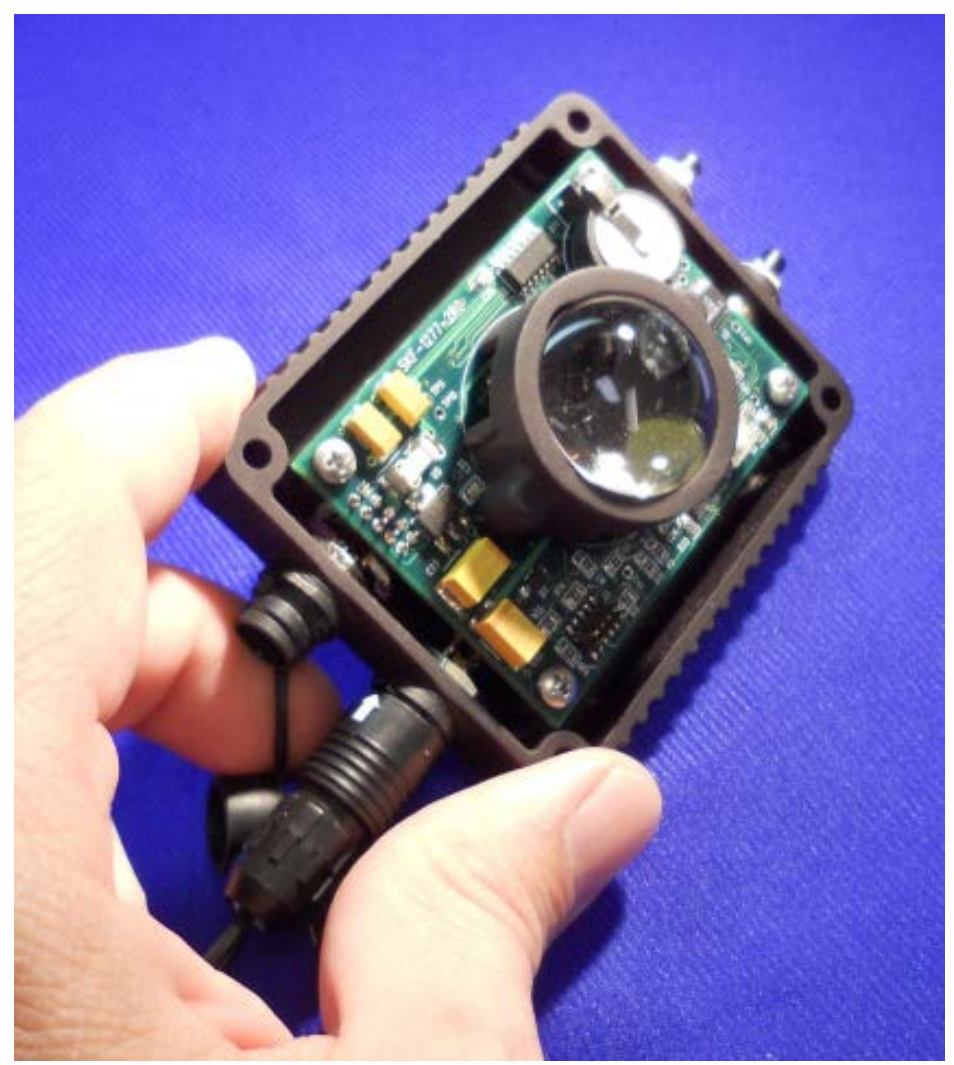

Fig. 43. Marker sensor internal electronics.

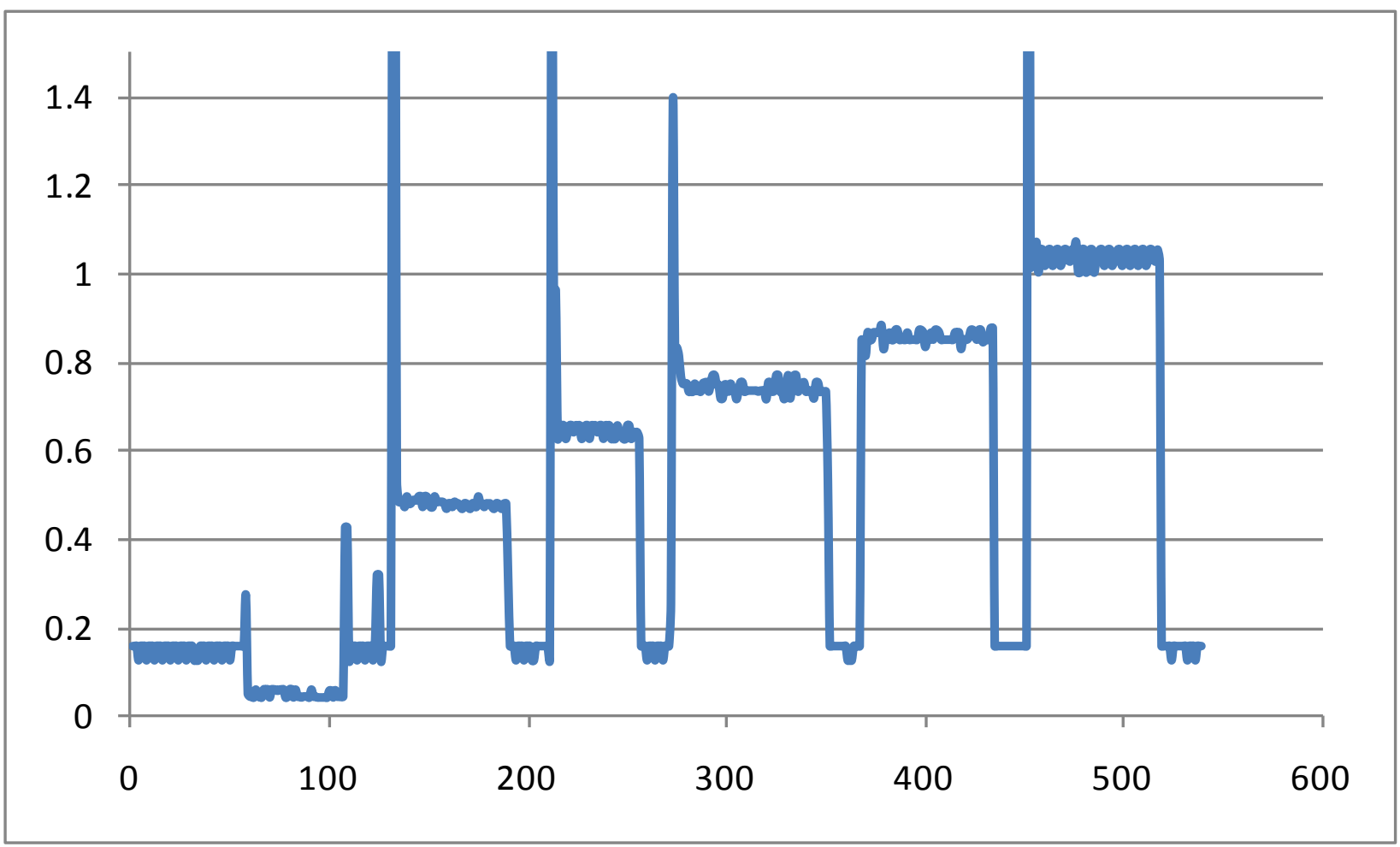

Fig. 44. Data from vials containing marked diesel fuel. 
Based on these early results, the performance of the newly developed fuel marker sensor was deemed acceptable and ready for use in a test track-based field test. The cost of the device in low-volume production was estimated at less than $\$ 100$.

\subsection{SENSOR SYSTEM LAYOUT AND CONFIGURATION}

\subsubsection{Approach}

In the initial phases of this project, collaborative agreements were pursued with a vendor that purported to design and market fuel tracking sensor systems. Another vendor was also identified that made similar claims. However, the first vendor was unable to provide any specifications or details that would enable consideration of their system, so attempts at collaboration were abandoned. The second vendor would not respond to phone and e-mail inquiries regarding their products. Therefore we resolved to develop our own mounting hardware for the sensor suite.

\subsubsection{Off-Trailer Evaluation and Development}

After careful consideration, a decision was made to mount all of the sensing hardware, with the exception of the hatch sensor, down by the fill valve of each compartment to be monitored, as shown schematically in Fig. 45.

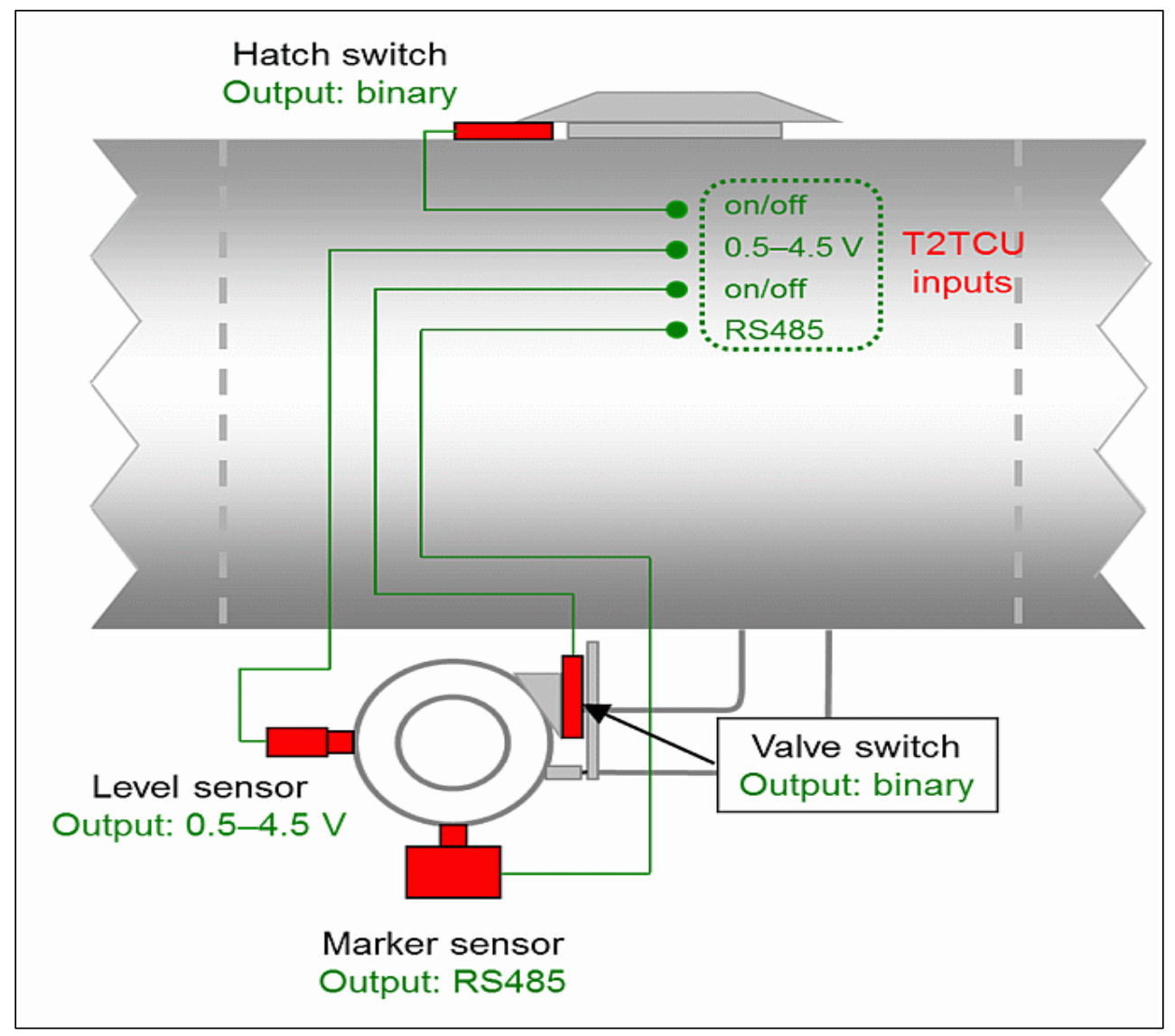

Fig. 45. Schematic of sensor placement around fill valve on trailer compartment. 
Further, since each downpipe leading from the compartment bottom to the fill valve already has a replaceable, acrylic sight glass, as shown in Fig. 46, it was decided to design a "drop-in” replacement sight glass that could accommodate the various sensors. In this section we will describe how the sensors were integrated with the sight glass and valve. See Appendix G for detailed drawings of the custom-made sight glass.

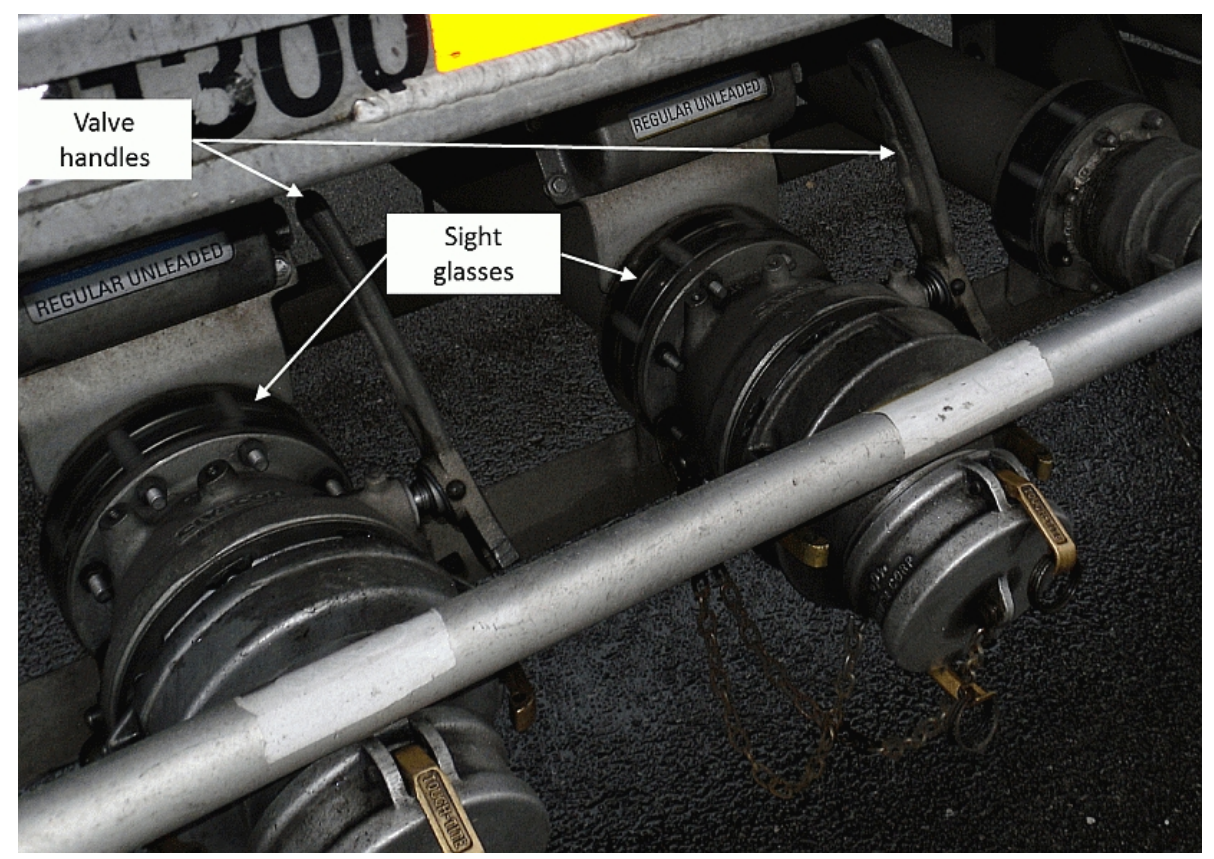

Fig. 46. View of downpipe region on trailer showing sight glasses and bottom valves.

Figure 47 shows how the sensors were arranged on the sight glass and valve. As discussed previously, the marker sensor (not yet installed in Fig. 47) mounts on the bottom of this sight glass. Figure 47 shows the isolation and bleed valves that were placed inline with the pressure-based level sensor, the isolation valve being upstream and the bleed valve downstream of the sensor. The isolation valve was primarily intended to protect the sensor in scenarios where high impulse pressure was anticipated. High impulse pressures were feared during fuel loading, when the fuel is pumped in using pressure supplied by the loading pump. A secondary purpose of the isolation valve was to isolate the sensor if leaking or other failure occurred. The bleed valve allows entrapped air or built-up pressure to be bled off as needed during the field test efforts.

The proximity sensor (valve switch) is mounted via a bracket that also serves to hold a pipe hanger for the level sensor. It was necessary to install a target magnet on the valve handle because the valve handles are cast aluminum and thus could not trigger the proximity switch.

With the sight glass and mounting hardware shown in Fig. 47, and the sensor development described above, the sensor team was able to offer a "drop-in" sensing suite for the field testing, a solution for monitoring the valve, the level of the fuel, and the marker in the fuel. Section 7 discusses what lessons were learned when deploying this sensor suite during the field testing. 


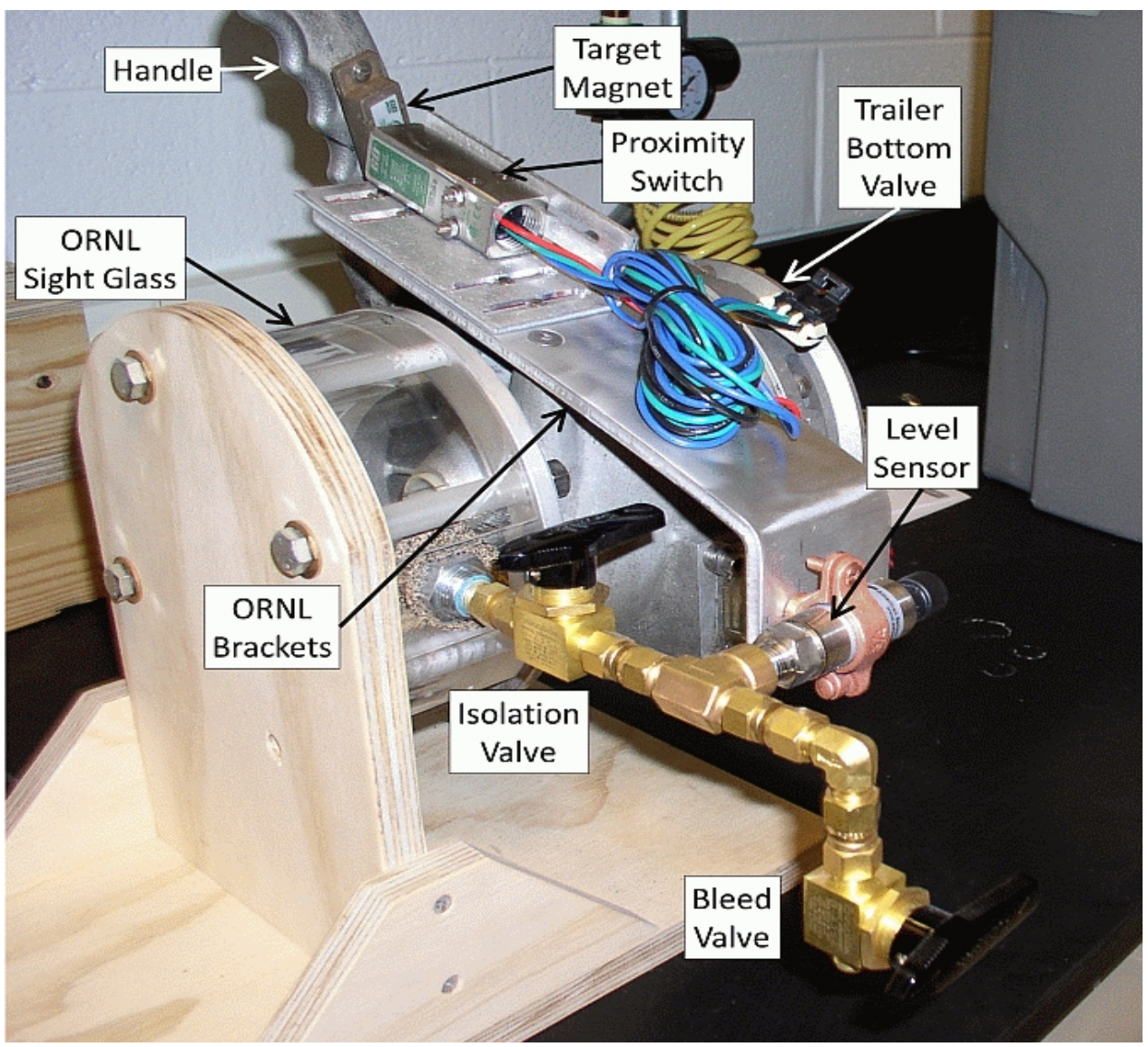

Fig. 47. Sensors mounted on trailer fill valve and ORNL-designed sight glass. 



\section{COMMUNICATIONS}

The communications portion of the FTE effort handled the real-time collection and transmission of onboard sensor data to the back office.

\subsection{SELECTION OF METHODOLOGY}

In the initial planning for this effort, it was thought that ORNL would use a wireless technology solution to transmit sensor data from the tractor to the trailer. Then, a computer-based DAS would be developed to gather and package the data for transmission to the back office via a novel or third-party land-based cellular system.

ORNL transportation staff were consulted about possible options based on their prior and ongoing heavytruck data collection and transmission work conducted for the Department of Energy's (DOE's) Vehicle Technologies Program and the Federal Motor Carrier Safety Administration's Wireless Roadside Inspection (WRI) Program. It was suggested that the work being done under these projects could be adapted and applied to the FTE project to reduce duplication of effort and provide better control of the data collection and transmission process by ORNL, which would be critical for the success of this phase of the effort.

The communications system required to accomplish the needs of the FTE effort would generally consist of the following major components:

1. Device to collect and format sensor signals from trailer (switches, level sensor, marker sensor, weight)

2. Device to convey the sensor signals from the trailer to the tractor without the addition of any cabling or wiring between the tractor and trailer (maintaining the integrity of the standard tractortrailer electrical connection was a requirement in order for the FTE solution to be universally accepted by stakeholders)

3. Device to receive sensor data from trailer and condition and format the sensor signals for acceptance by the on-board computer

4. Communications platform capable of sending the data packets to a back office system (BOS) for processing

The basic conceptual components of the FTE system are shown in Fig. 48.

Two concepts for data collection and transmission were proposed: (1) use currently existing cellular telematics solution such as an electronic on-board recorder or (2) use the DAS and communications systems developed by ORNL for the WRI POC testing. These methods will be coved in Sects. 4.1.1 and 4.1.2. For either of these transmission methods to be successful, an effective means of communicating the sensor data from the trailer to the tractor would need to be identified or developed. This will be covered in detail in Sect. 4.2.

\subsubsection{Use of Currently Existing Telematics Device}

There currently exist a number of off-the-shelf technologies to retrieve data from commercial vehicles. These systems, called "telematics devices," consist of an on-board computer, an interface to the vehicle data bus, optional external input ports, a display screen, a keyboard or other input device, and wireless communications capability to transfer information from the vehicle via commercial mobile radio services. Such devices are used by the trucking industry to communicate information to and from the driver, track 
vehicles and cargo, record driver hours of service, and monitor vehicle systems and vehicle performance for maintenance and safety personnel.

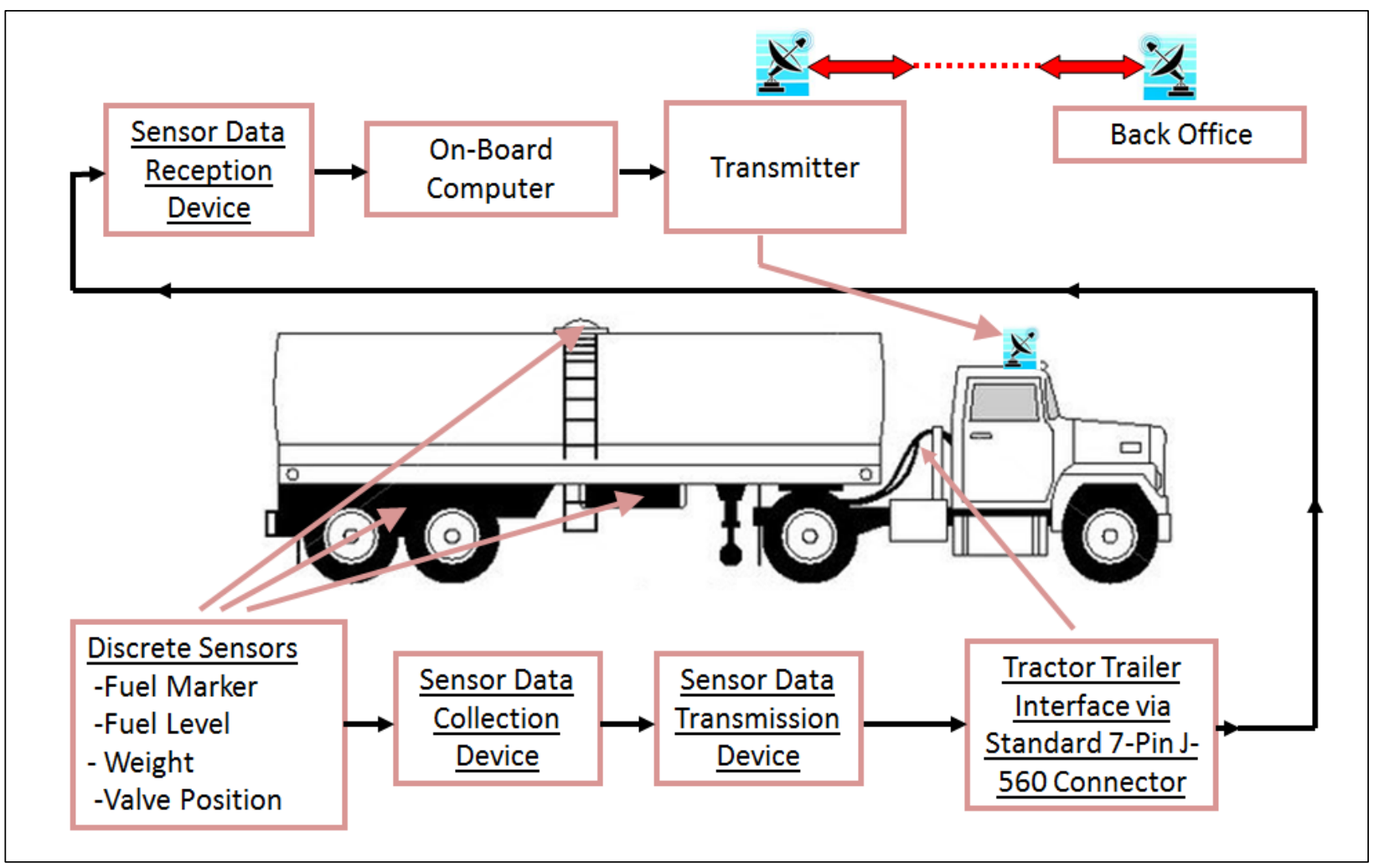

Fig. 48. Conceptual components of the fuel-tax-evasion system.

In order to use a currently existing telematics device, the sensor data from the vehicle would need to be brought to the tractor (the location of the telematics computer), be accepted by the telematics device (input port available, data format, and data sample rate), be stored by the device, and be packaged for transmission by the device. Additionally, the telematics supplier's back office would need to be able to receive and parse the additional data for this effort and transmit it via the Internet for use by ORNL.

ORNL had previously conducted a survey of the capabilities of existing telematics hardware and systems for the WRI effort and contacted vendors whose systems seemed promising for the FTE project.

However, the required sample rate and RS485 communications defined for the FTE project's fuel marker sensor proved to be problematic to the telematics providers. While their systems could be adapted to facilitate the needs of the FTE project, considerable time and resources would be required. Additionally, there were risks associated with the development of the needed software application. It was decided to use the ORNL DAS developed for the WRI project because it provided the reliability and flexibility needed for this early stage of system testing and development. Once a successful field test was conducted using the ORNL DAS, a set of functional specifications for the system could be developed and shared with the telematics industry for their use in supporting future FTE efforts.

\subsubsection{Use of ORNL DAS}

To circumvent the identified issues relative to the use of a standard telematics solution (acceptance of sensor signals, fast sample rate, data buffering, connection to the vehicle's data bus), it was decided to use 
the DAS and communications systems developed by ORNL for the WRI POC testing (Fig. 49). This system was readily able to connect to the vehicles data bus (older J1708 and/or the newer J1939) and was able to sample the data at rates up to $1 \mathrm{hertz}(\mathrm{Hz})$ while buffering data for extended periods of time if necessary. Additionally, this ORNL DAS had a built-in cellular modem to allow the data to be wirelessly transmitted to a webpage for manipulation by the evidential reasoning system.

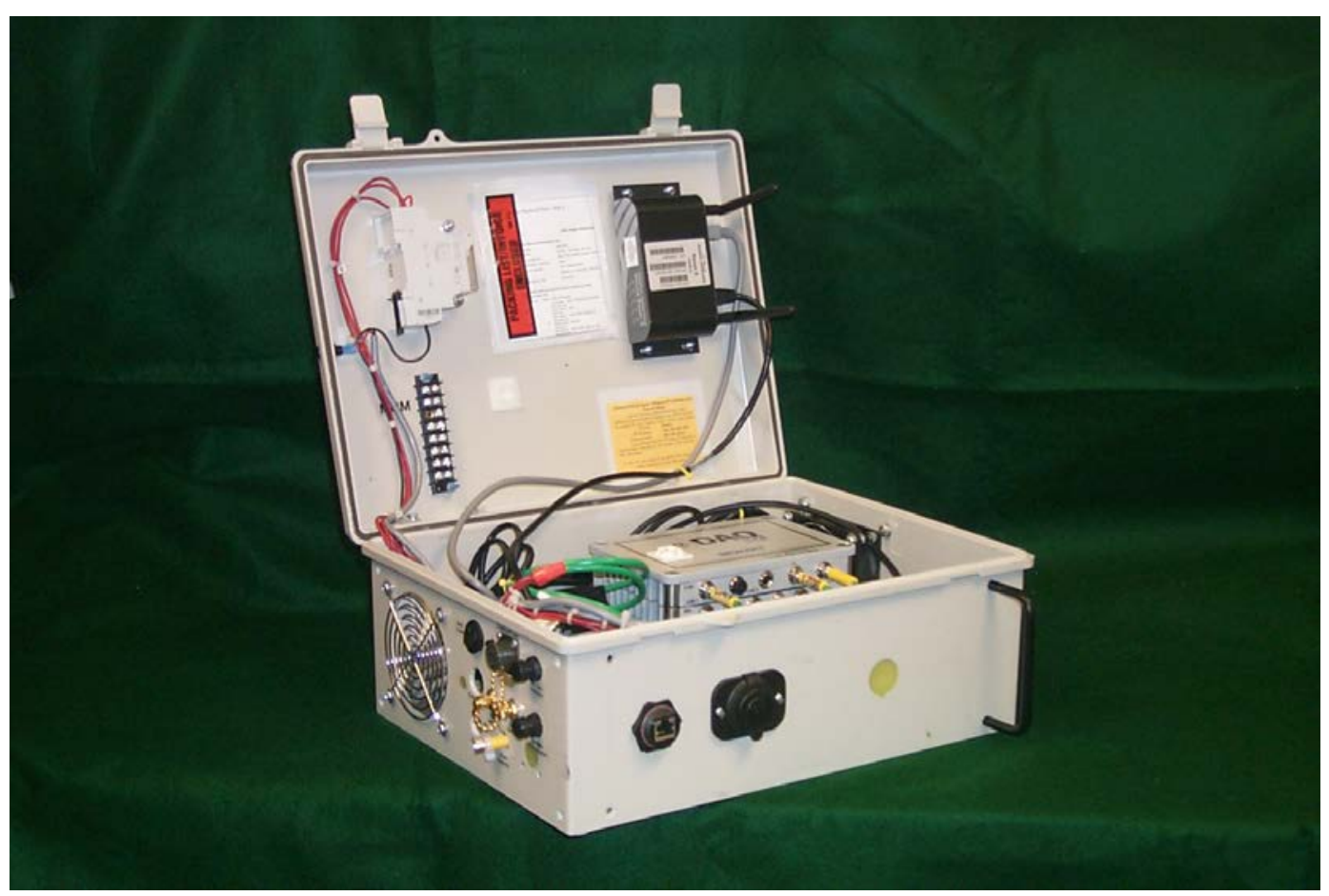

Fig. 49. ORNL DAS used for data collection.

With the ORNL DAS's ability to connect directly to the vehicle data bus, it was decided to post the trailer sensor data to the tractor's J1939 data bus using Frequency Shift Key (FSK) signaling. FSK signaling allows for the transmission of digitized data from the trailer-borne sensors to be passed from the trailer to the tractor over the standard J560 electrical connector. An FSK modem converts the digital data (modulated in the frequency domain) across the J560 connector, and a mating FSK modem on the trailer coverts the frequency domain signal back to digital data (demodulation). This novel approach "piggybacks” on the existing trailer-to-tractor wiring and requires no additional connections.

The major components of the proposed FTE data communications system were as follows:

- Trailer-to-Tractor Communications Unit (T2TCU), consisting of a module to receive the sensor signals, an FSK modem modulator to send sensor data over the J560 connector, FSK modem demodulator to convert sensor data back to digital information, and a module to place sensor data onto the tractors J1939. The T2TCU set of components are located both on the tractor and trailer.

- ORNL DAS with cellular model for wireless data transfer.

These major components are shown in Fig. 50. 


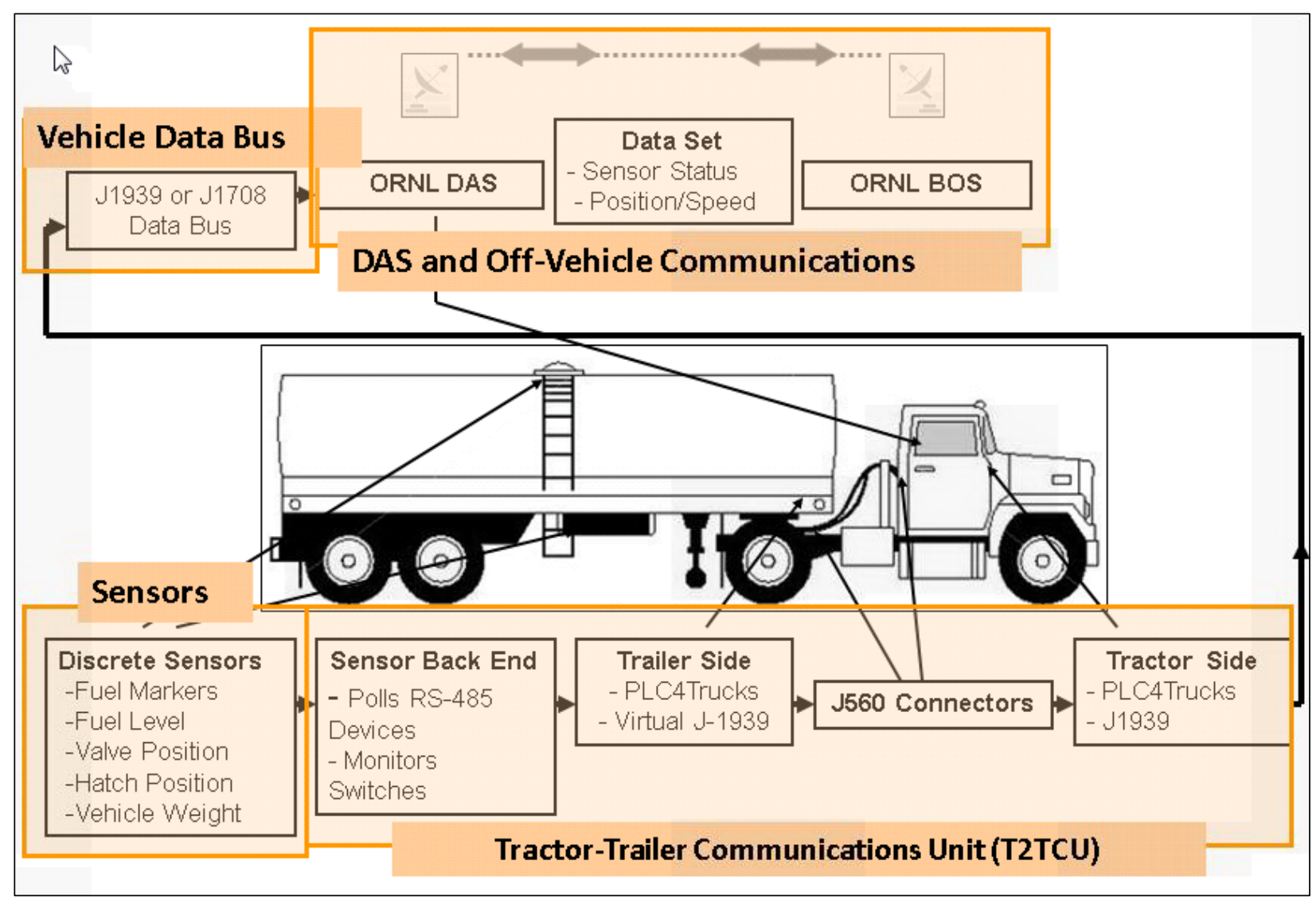

Fig. 50. Fuel-tax-evasion communications major components.

\subsection{DESIGN OF THE T2TCU}

Due to the restrictions imposed by the use of the RS485 protocol for the fuel marker sensor, a suitable commercial off-the-shelf solution was not identified for the T2TCU. Therefore, the decision was made to draft a set of functional specifications for such a device and to seek bids from private industry to supply the T2TCU in a hardened form for use in the field testing. Air-Weigh, Inc. (Air-Weigh) of Eugene, Oregon, was awarded the contract to design and build the T2TCU for use in the FTE project.

The T2TCU was developed with the following inputs in mind:

1. RS485 fuel marker sensors (2),

2. Hatch position sensors (2),

3. Belly valve position sensors (2),

4. Fuel level transducers (2),

5. Trailer weight transducer (1), and

6. Tractor weight transducer (1).

Air-Weigh proposed modifying their already-existing eStroke Chassis Communications Module (CCM), an FSK-based device, to facilitate the needed sensor data collection, transfer the data from the trailer to the tractor, and post this information onto the tractor's J1939 data bus. This device was already capable of handling the weight transducer signals as well as posting sensor data onto the tractor's J1939 data bus. Air-Weigh's design team met the additional requirements of the FTE effort by performing the following work steps: 
1. Modified the CCM device to collect data from hatch, valve, liquid level, and marker concentration sensors. The modification consisted of hardware changes for the device to operate in a trailer environment exposed to outside weather conditions, hardware changes relative to sensor connectivity, and software to interface the CCM unit to the sensors. This modified device was identified as T2TCU Trailer.

2. Modified the CCM software such that the T2TCU Trailer device will transmit the data as collected to a corresponding an Air-Weigh LoadMaxx tractor unit (identified as T2TCU Tractor) over the standard tractor-trailer J560 power/signals connector.

3. Modified the LoadMaxx software to serve as a T2TCU Tractor device to receive the data from the T2TCU Trailer device and transmitted this data to the vehicle J1939 data bus.

4. Provided three sets of modified hardware for the project. Each set of hardware consisted of a modified CCM device with sensor and power cables and a modified LoadMaxx device with power and interface cables. The three sets of hardware were used for

(a) Lab Testing by Air-Weigh (non-environmentally hardened system),

(b) Lab Testing by ORNL (non-environmentally hardened system), and

(c) Field Testing on a vehicle which included a tractor and trailer (environmentally hardened system).

5. Provided assistance to ORNL in testing and identifying J1939 parameters.

The trailer side of the T2TCU provided power to the sensors installed for each instrumented compartment. The T2TCU transmitted the sensor readings constantly at a rate of $1 \mathrm{~Hz}$; no data accrual or additional on-board decision-making was required. Communication between the tractor and trailer was done via the standard J560 connection native to the vehicle. The tractor-side information interface was done via the J1939. The T2TCU is shown in Fig. 51, along with a few sensors used in benchtop testing.

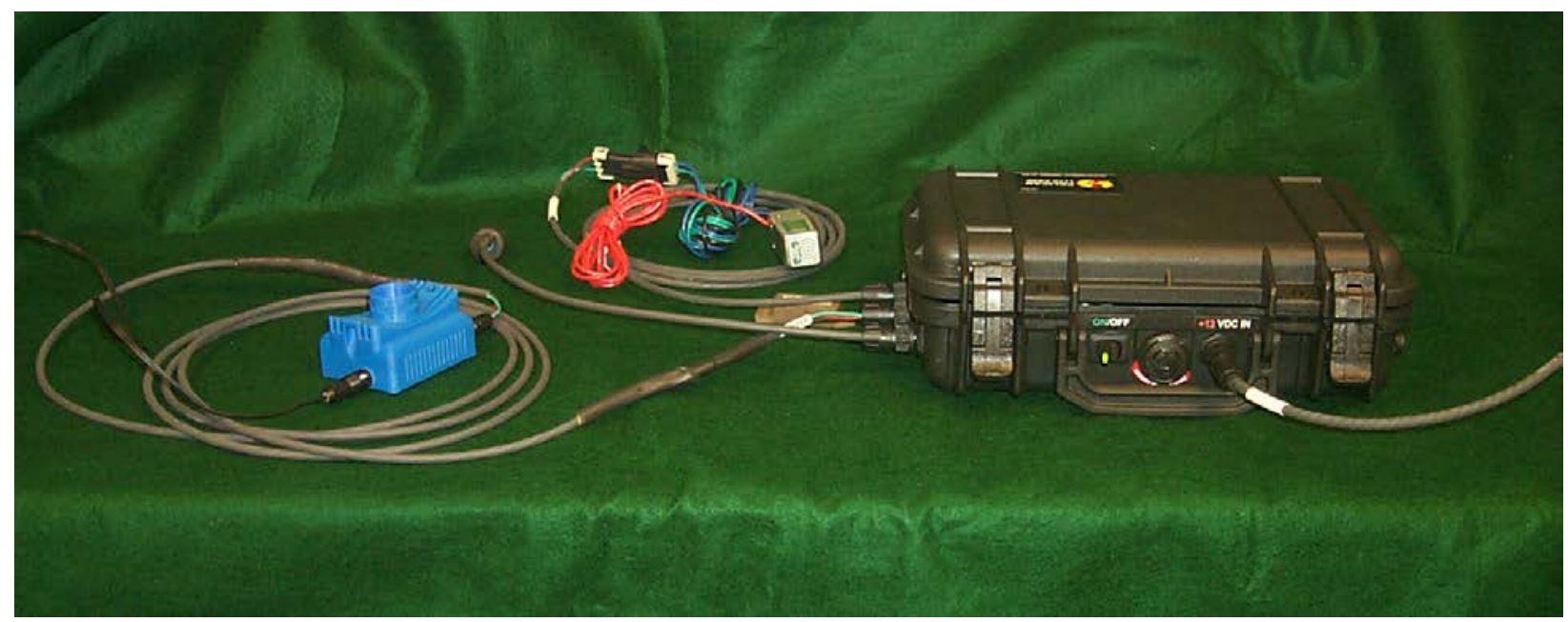

Fig. 51. T2TCU with test sensors.

Further, Air-Weigh was tasked to provide support for installation of the T2TCU equipment on a tractor and trailer, support ORNL during the field testing, and assist ORNL in preparation of the final report as needed.

A list of the sensors along with their corresponding measurements and power requirements is shown in Table 5; two tanker compartments were instrumented for a total of eight sensors. 
Table 5. Summary of sensors per fuel tanker compartment

\begin{tabular}{|c|c|c|c|c|}
\hline Sensor & Type & Measurement & $\begin{array}{c}\text { Power } \\
\text { requirements }\end{array}$ & Other information \\
\hline Hatch & SPDT switch & Open/closed status & $5-12 \mathrm{~V},<5 \mathrm{~mA}$ & \multirow{2}{*}{$\begin{array}{l}\text { No current draw ("open”) } \\
\text { when hatch/valve is closed }\end{array}$} \\
\hline Valve & SPDT switch & Open/closed status & $5-12 \mathrm{~V},<5 \mathrm{~mA}$ & \\
\hline Liquid level & $4-20 \mathrm{~mA}$ & Pressure $(\mathrm{kPa})$ & $12 \mathrm{~V}, 20 \mathrm{~mA}$ & Calibration capability needed \\
\hline $\begin{array}{l}\text { Marker } \\
\text { concentration }\end{array}$ & RS485 & $\begin{array}{l}\text { Dimensionless Parameter } \\
(0-1)\end{array}$ & $12 \mathrm{~V}, 300 \mathrm{~mA}$ & $\begin{array}{l}\text { See manufacturer's appendix } \\
\text { for details (not included in } \\
\text { this document) }\end{array}$ \\
\hline
\end{tabular}

\subsection{DATA RETRIEVAL}

The data posted by the T2TCU was read by the ORNL DAS at $1 \mathrm{~Hz}$. A real-time communications link was established between an ORNL computer and the DAS which was made possible by the on-board cellular modem. This streaming data was monitored and split into short files capturing 5 min increments of data; these files were made available to the evidential reasoning component for analysis via automated uploads to a server.

The FTE Upload Data Service (data retrieval) software system developed by ORNL presented the user with the screen shown in Fig. 52 when the software was started. The user had the choice to specify the data saving frequency either by checking the box labeled "One-second Data Record" on the main screen or by specifying data sampling parameters. The former would force the software to retrieve and save the real-time data at a $1 \mathrm{~Hz}$ frequency.

If a different data saving frequency was required, the user could have specified this, and other parameters, by clicking on the "Change Settings" button located on the upper-right corner of the main screen. When clicking this button, the software showed a dialog box for the user to enter his/her password to determine whether that user had enough privileges to change the data retrieval settings.

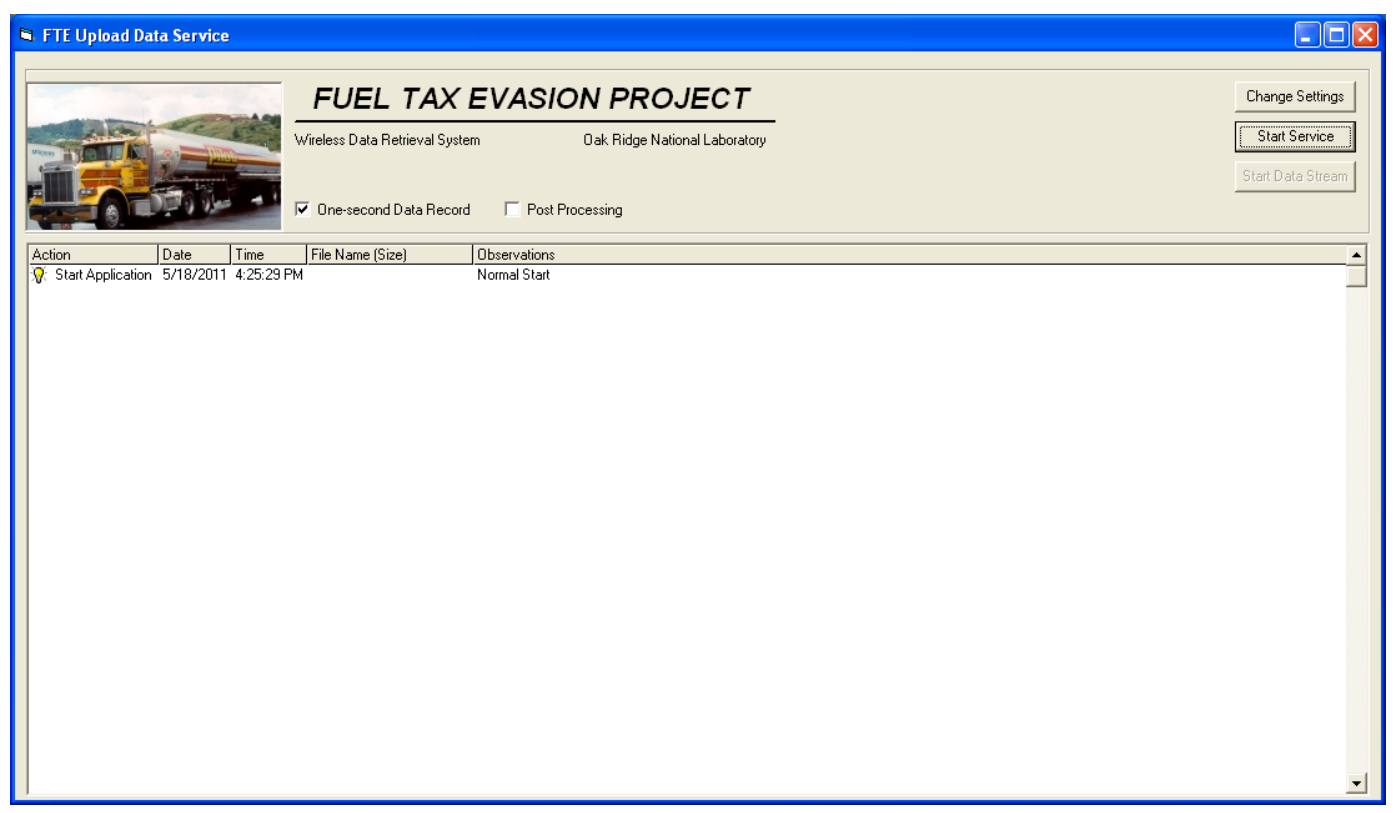

Fig. 52. Fuel-Tax-Evasion Upload Data Service software. 
Once the password was validated, the software presented the user with the "FTE Settings" dialog box shown in Fig. 53. In the "Data Sampling Information" frame (lower-left corner), the user could specify the sampling and data saving frequencies, as well as other parameters such as the length of the reporting period (5-min data files was the default). The "FTE Settings" dialog box also allowed the user to specify information describing the FTE vehicles and the IP addresses of the on-board DAS. In cases where communication was lost, or if there were any other problem detected by the FTE Upload Data Service software, a message was e-mailed to the address specified in the "Communication Information" frame within the "FTE Settings" dialog box.

After all the data retrieval and saving parameters were specified, the user pressed the "Start Service" button (Fig. 52). The software then started the data service, indicating this event on the main screen (Fig. 54). At that point, the "Start Service" button changed to "Stop Service" to allow the user to terminate the data-retrieval and saving service.

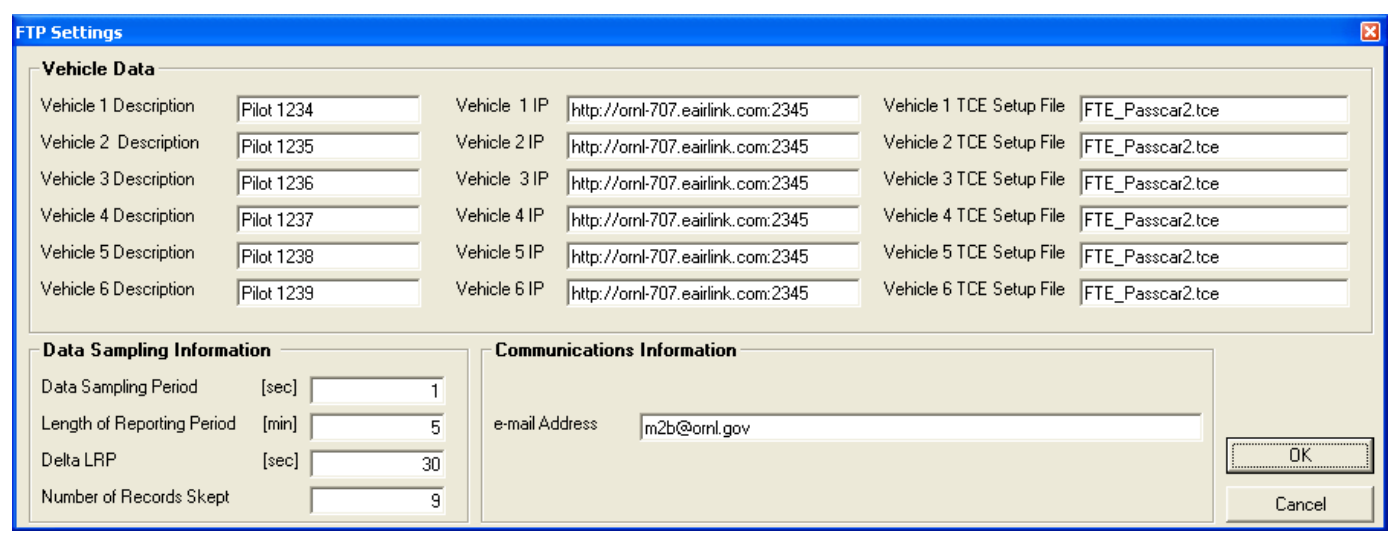

Fig. 53. Fuel-Tax-Evasion Upload Data Service software-FTE settings.

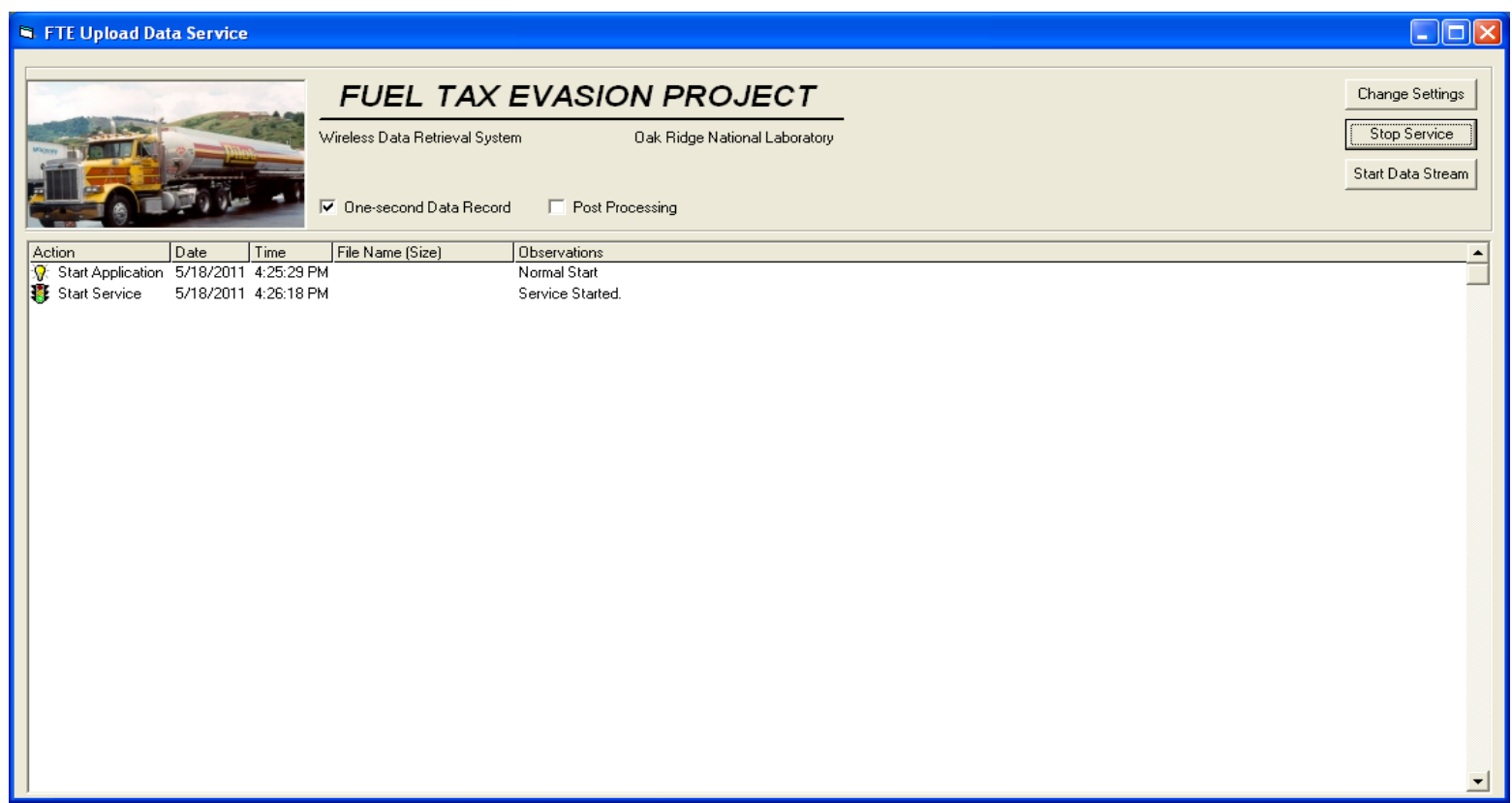

Fig. 54. Fuel Tax Evasion Upload Data Service software—started data retrieval service. 
Once the user was ready to start the actual data collection, the "Start Data Stream" button located in the main screen was pressed. This triggered the communication software to establish communication with the vehicle(s) participating in the tests and start retrieving, processing, and saving the real-time information generated by the vehicle(s). Figure 55 shows the communications software accessing one of the vehicles that participated in the test; note that the communications software used was publicly available software named "curl."

The FTE Upload Data Service software could also be used to post-process the data files (note: the onboard DAS also saved the data collected in files that could be post processed after the tests were concluded). This was achieved by checking the "Post Processing" check box on the main screen and pressing the "Start Service" button (Fig. 52).

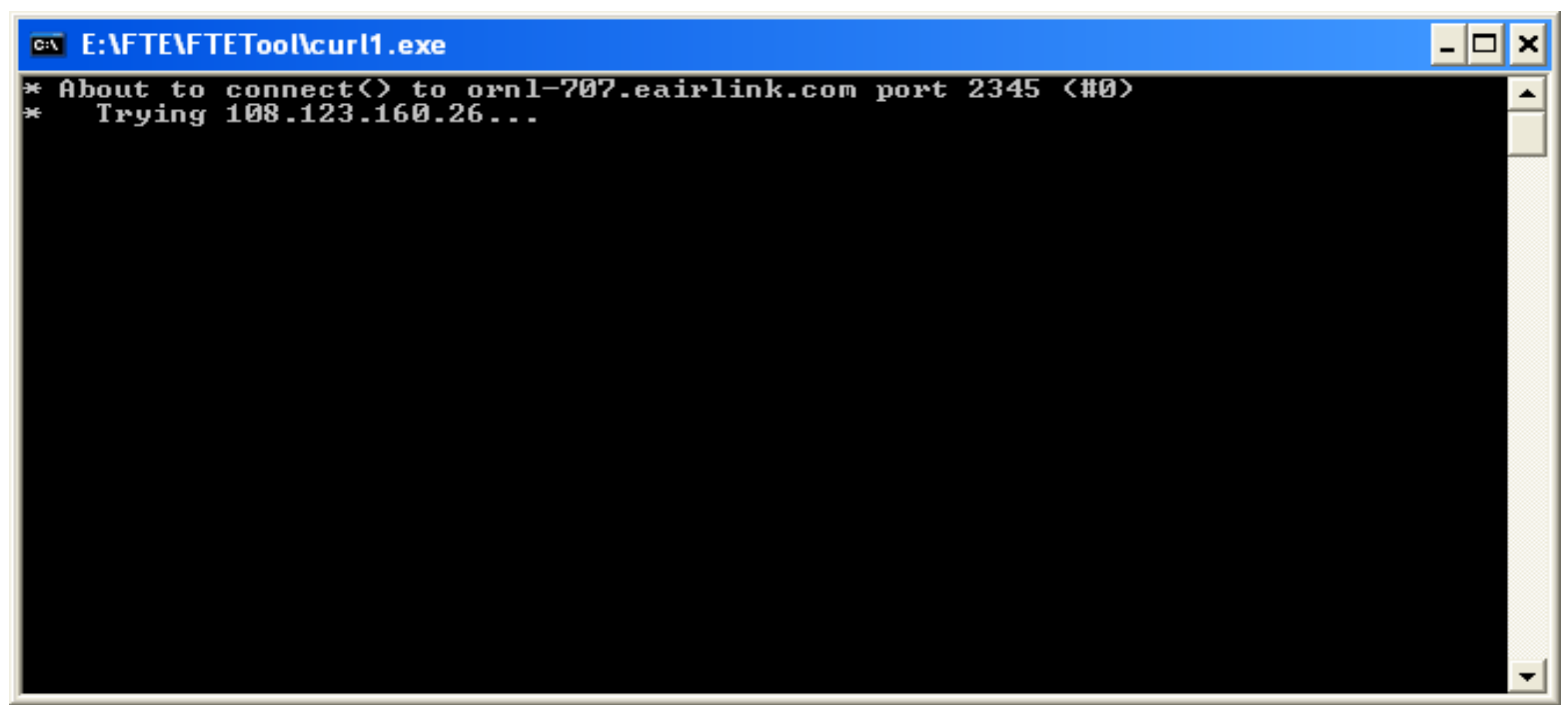

Fig. 55. Fuel-Tax-Evasion Upload Data Service software—communications software. 


\section{GEO-FENCING AND EVIDENTIAL REASONING APPLICATIONS}

As part of this research study, the development of a POC intelligent GIS-based system was called for. The system aims to facilitate near-real time notification of suspicious variation in standard delivery processes, tracking the tanker as it travels from the origin (e.g., terminal) to its destinations (e.g., retail outlet) on the delivery route. The control center, where the "system" resides, monitors sensor activities occurring at and between the terminal loading of the tank truck and the unloading at the retail outlet. By applying sensor technologies in combination with tools developed based on data fusion and validation techniques, suspicious activities can be identified in real time. When necessary, communication with law enforcement personnel can be performed in a timely manner to facilitate the investigation of potential fuel diversion activities.

The intelligent GIS-based system developed under this research study performs two major functions: geofencing and evidential reasoning. Description of these techniques, general uses, and their specific roles applied within the scope of this study are covered in this section.

\subsection{BACKGROUND}

\subsubsection{Geo-Fencing Basics}

A geo-fence is a virtual perimeter for a given geographic area, for example, a point location or a region. The geo-fence could be set with a predetermined set of boundaries (e.g., school zone) or be dynamically generated (e.g., within a 2 mile radius of the point location). The geo-fencing capability is a critical element in the telematics system's functionalities, which is commonly used in the trucking industry (e.g., PeopleNet) for vehicle tracking and monitoring. When a geo-fence is crossed by an equipped vehicle (as determined from GPS data), a warning is triggered. This warning can then be transmitted electronically (e.g., email or other communication) to the designated person (e.g., operator at the control center or enforcement personnel). In certain extreme situations, the geo-fencing can be linked to technology that immobilizes the vehicle (such as in the case of a stolen vehicle).

Under this research study, instead of geo-fencing at a point location (e.g., the gas station) as is typically provided by telematics providers, a buffer zone along the truck travel route is considered (Fig. 56). A discussion of the development of a geo-fencing application, related tracking methodology, and challenges encountered in this project are provided in Sect. 5.3 of this report.

\subsubsection{Fundamentals of Evidential Reasoning Methods}

Under this POC study, the evidential reasoning approach is to be integrated into an intelligent threat identification and assessment GIS-based system to assist an analyst in sorting through volumes of data and information (i.e., evidence). This system would flag any peculiar patterns or suspicious activities, so proper responses and timely actions can be taken.

Evidential reasoning techniques are commonly used in knowledge-based expert systems and artificial intelligence researches. The objective of this technique is to assess the effect of all available pieces of evidence upon a hypothesis, by making use of domain-specific knowledge. Various techniques can be applied to perform evidential reasoning tasks, each with several variants depending on their applications. Many discussions of reasoning frameworks can be found in literatures, for example, to name only a few, Valin, ${ }^{6}$ Yang \& Xu, ${ }^{7}$ and Tang \& Al-shaer. ${ }^{8}$ Two specific types of evidential reasoning applications are implemented in this current FTE detection research study; they are the rule-based logic reasoning technique and the Dempster-Shafer (D-S) believe function-based approach. A brief introduction of these two reasoning approaches is given below. 


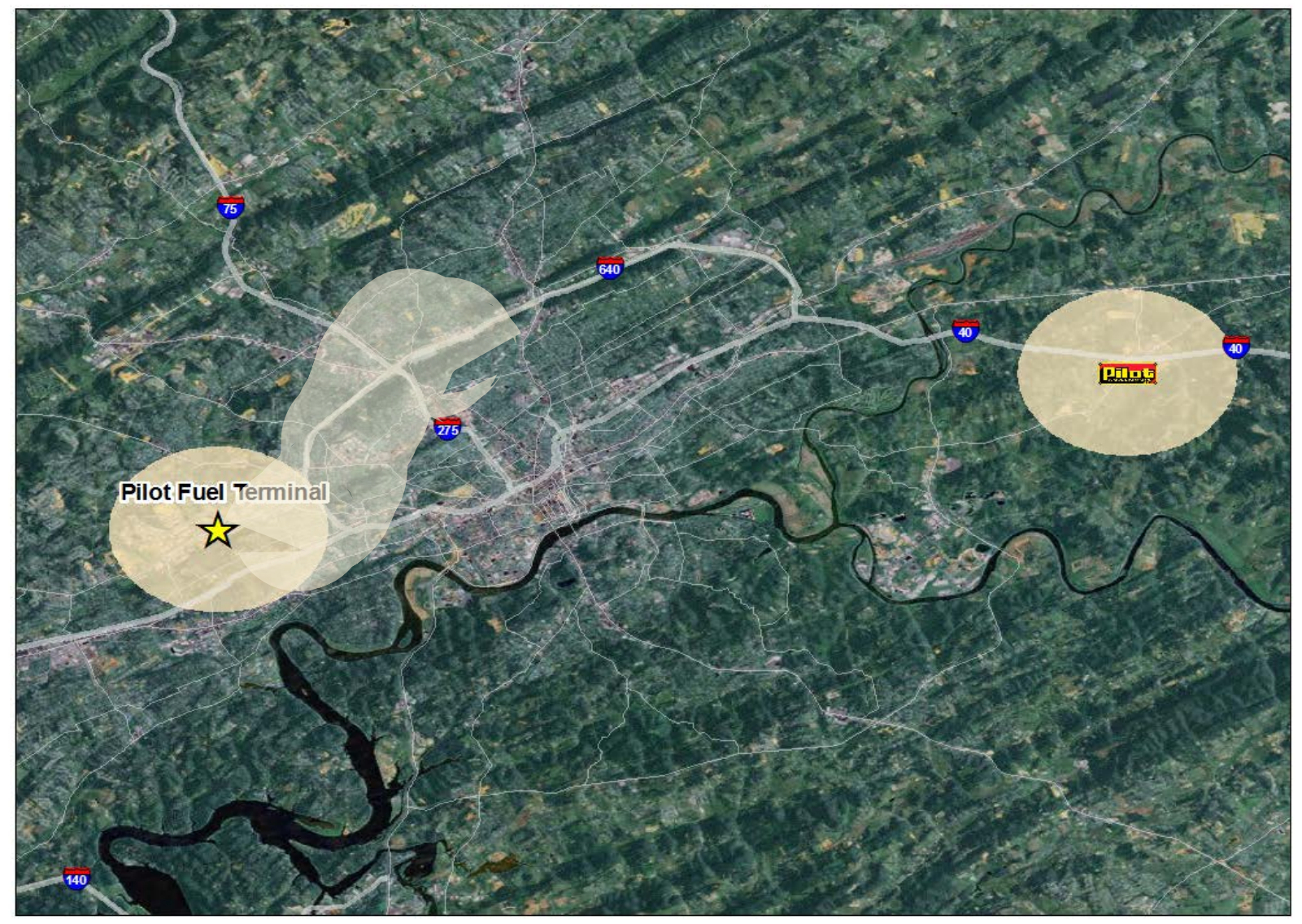

Fig. 56. Example of a geo-fencing proximity.

\subsubsection{Rule-based logic reasoning}

Rule-based reasoning approach is based on simple logic of if-then rules. This approach has obvious computational advantages. These if-then rules typically can be established to reflect logic and patterns recognized from examining existing datasets (e.g., relevant historical data). In many cases, particularly when formal data is not readily available, knowledge from subject experts can also be captured to derive decision rules. For complex situations, however, an excessive amount of expert knowledge will be required in order to have a comprehensive set of rules to effectively perform the necessary reasoning. This is one of the disadvantages of this type of reasoning approach. Furthermore, information based on knowledge of experts could be quite subjective, and combining multiple sets of knowledge from a group of experts to draw a consensus might not necessarily be trivial.

Moreover, the rules are commonly fixed and exact (i.e., if A then B). Therefore, uncertainty is generally not considered. To allow limited consideration of uncertainty in this reasoning approach, researchers have included fuzzy logic in some rules. In this case, instead of the exact "true or false” when evaluating a rule, fuzzy logic variables can be set to have a range of values based on the degree of likelihood (i.e., from 0 to 1$)$. 


\subsubsection{Dempster-Shafer evidential reasoning techniques}

Dempster-Shafer (D-S) theory is a powerful technique of combining accumulative evidence or for changing prior opinions in the light of new evidence. It is well known for its usefulness to express uncertainty and, at times, conflicting judgments (such as knowledge gathered from a panel of experts). The D-S theory, although a probabilistic approach, not only allows for a measure of uncertainty but also takes into account a proposition's ignorance of which hypothesis is correct. This is the main difference between probability and the D-S reasoning (which defines measures as "beliefs"). For example, in probability theory, the probabilities of mutually exclusive hypothesis must sum to 1 , so if the probability of "snow" is 0.6, it implies that the probability of "not snow" is 0.4 (i.e., $1-0.6$ ). On the other hand, D-S beliefs for mutually exclusive hypothesis may sum to less than 1 . For a given event $A$, the D-S measures are defined as follows:

- $\quad$ Belief $(A)$, which represents the lower bound (pessimistic)

- $\quad$ Doubt $(A)=$ Belief $(\operatorname{not} A)$

- Plausibility $(A)=1$ - Doubt $(A)$ which represents the upper bound (optimistic)

The capability to model the "doubt” makes D-S belief theory a prevailing choice for many researchers and applications. That is, D-S approach allows "incomplete" assessments to be conducted when situations such as a lack of data or the inability of experts to assess or provide precise information occur.

Essentially, the D-S belief network can be viewed as a graphical and numerical representation of dependences and uncertainty. A belief network is a computational structure composed of clusters of nodes representing variables (e.g., evidence) interrelated by links signifying the relationships among the nodes. It is frequently used as a knowledge representation scheme to handle situations where causal or diagnostic relationships are to be captured and reviewed.

Various forms of D-S evidential reasoning approaches have been applied in classification of image texture for remote sensing applications, in detection of insurance fraud, in system safety and reliability modeling, and in many other applications. Since the D-S evidential reasoning approach uses beliefs to allow an "agent" (i.e., software or algorithm) to act rationally, it is more likely to produce diagnoses correctly and more quickly than other evidential reasoning techniques.

\subsubsection{Challenges}

In an ideal setting, the "legitimacy" of individual loads, offloads, and movements of petroleum products can be identified by integrating and validating, through evidential reasoning processes, information from electronic shipping documentation, vehicle tracking devices, sensor devices, and fuel markers. Suspicious activities can then be "flagged" and appropriate actions can be taken by the control center personnel (e.g., contact driver, inform enforcement).

Several challenges were encountered during the course of the project that limited the development of "ideal” functionality of the intelligent GIS-based system. Although Pilot provided the Evidential Reasoning Team access its PeopleNet tracking reports (data collected from on-board telematics devices), no shipping documentation or information from dispatcher scheduling (about the deliveries) was available. Available PeopleNet reports allowed the Evidential Reasoning Team to geographically trace Pilot truck travel routes, stop locations and durations, time and dates, and similar information. However, there was no information available regarding fuel quantity being handled (loaded or offloaded) at any locations, whether a particular stop was for a scheduled delivery or for other purposes, or any information about actual activities being conducted at the given locations. 
Another major limitation was the availability of sensor data (for valve, hatch, fuel level in the tank, and fuel marker concentration). Since no Pilot trucks (nor the passenger car used for simulation) were actually instrumented with the sensors, the Evidential Reasoning Team had to rely on very limited simulation data for its modeling and algorithm design and development. Additional information on the system design and development is given in this section.

\subsection{REAL-WORLD FUEL DELIVERY OPERATION DATA COLLECTION EFFORTS}

Data related to fuel delivery operations was obtained from several sources including observations made during "shadowing" of tanker drivers during normal operations, notes gathered from interviews with Pilot managers, and analysis of specific PeopleNet reports. Supplemental information was also collected through web searches and internal communications with the Sensor Team. In addition, simulations were conducted to generate sensor information using a passenger car with connections to the DAS. These data sources and summary findings are briefly described in this section.

\subsubsection{Real-World Information Relating to Pilot Tanker Truck Operations}

Information regarding Pilot tanker truck delivery operations was gathered from observations made by the Evidential Reasoning Team members during trips shadowing the tanker drivers as well as from discussions with Pilot managers. Summaries from these information collection efforts are presented in the following subsections.

\subsubsection{Observations from Pilot fuel delivery operations}

The Evidential Reasoning Team made two visits to the Pilot tanker truck terminal located in Knoxville, Tennessee. Several trips were made in which the team shadowed Pilot tanker truck drivers. The first visit focused on activities where tanker trucks conducted the fuel loading, while the second visit were to observe all fuel-delivery-related activities. During their time with the drivers, the Evidential Reasoning Team acquired some useful information on how the trucks operate. This section describes what the team observed from the entire pickup (i.e., loading from a terminal rack) and delivery processes (i.e., offloading fuel at a delivery location).

\section{General operations}

Pilot does not own fuel terminals, although Pilot might own the fuel that is stored at the terminal. The team learned that Pilot gas stations and Travel Centers (i.e., gas stations with convenient stores) are not individually owned franchises; rather, they are owned and operated by the Pilot Travel Center LLC (currently, Pilot \& Flying J LLC) Corporation. Thus, all gas station/store employees are Pilot employees. All fuels sold at Pilot gas stations are delivered by Pilot's own tanker trucks. In addition to delivering fuel for Pilot travel centers and gas stations (which now includes Flying J gas stations), Pilot tankers also make fuel deliveries to other gas stations, truck stops, and wholesale customers. These non-Pilot-owned gas stations could also utilize multiple companies to deliver their fuels.

Although, Pilot fuel tankers operate 24 hours a day, 7 days a week, their drivers are on a 12-hour work shift, operating under the required DOT hours-of-service rule. The on-board system serves as driver's logbook and provides automatic tracking information (active on-duty hours and active driving hours) for the driver. In terms of daily schedule, the drivers receive their list of deliveries from the dispatcher in the morning. The dispatchers' list is generated hours in advance, typically based on estimates of gas sales from the day before. Usually a driver makes about four to five pickup and delivery tours per shift. One of the Pilot drivers being shadowed for this visit is a "Team Leader" driver. The "Team Leader" reports directly to the fleet manager and usually has a group of Pilot tanker drivers that report to him. 
The Pilot drivers use both PeopleNet and cell phones for communication. PeopleNet allows text communication (messaging) among the Pilot tanker trucks, the fuel terminals, Pilot headquarters, and Pilot Travel Centers (gas stations). While the dispatcher can reroute a tanker to make an "unscheduled" delivery if the need arises, the driver can also ping a gas station prior to a "scheduled" delivery to obtain more up-to-date fuel inventories by fuel types, so the need of delivery can be confirmed. That is, the Pilot driver has the ability to double-check prior to traveling to the gas station, to determine whether the station actually has the storage capacity for the amount of fuel that was scheduled to be delivered. If the needed storage capacity is not available, the driver will communicate with the dispatcher to obtain other delivery assignments. Drivers can get more up-to-date inventory data by communicating with the gas station system right before its scheduled delivery.

It is not unusual for the drivers to have last-minute schedule changes. One driver indicated that his delivery schedule for that morning had changed twice already before the Evidential Reasoning team shadowing exercise started in that morning. Also during one of the shadowing exercises, a driver received an urgent message from the dispatcher regarding a non-Pilot store that was out of gas. The dispatcher asked the driver to make this station his next delivery. According to the driver, this situation would not happen to a Pilot-owned gas station or travel center because the Pilot system tracks inventories for all the stores they own. Non-Pilot owned stores are responsible for checking their own inventories.

\section{Equipment (tanker truck) operations}

Typically, Pilot tanker trucks are equipped with four compartments; the first and the fourth compartments have capacities of 3,000 gal, and the middle two compartments are 1,500 gal each. In practice, drivers usually do not load the compartments up to their full capacities, however. For instance, one of the drivers being shadowed only filled about 2,500 gal each into the two 3,000 gal compartments and about 1,300 each into the other two 1,500 gal compartments, which is roughly about $85 \%$ capacity. The reason provided by the drivers was that, under current DOT highway law, the overall gross vehicle weight of the truck is not to exceed 80,000 lb. Therefore, drivers would either fill the tanker compartments partially full (as in the given example) or load only two or three of the four compartments.

There are hatches on the top of the tankers, but no "top" loading is allowed due to EPA ruling. The hatches are typically used during maintenance of the tankers. However, hatches can be opened for monitoring the fuel intake (which requires two people) on tankers that do not have an automatic tank-full stop mechanism installed. There is no flow meter equipped on the tankers, even though one driver claimed that he can "time" the opening and closing of the valve to "guesstimate" how many gallons of fuel are being transferred. This is the main reason why drivers do not want to off-load partial loads from the tanker compartments.

\section{Fuel loading operations}

Before starting the tanker, the driver usually make a visual inspection of the truck by walking around it to ensure that no obstacles could hinder the truck's free movement, and some also checked all tires. At times, the line at the fueling terminal could be long, so the driver would have to wait a while before pulling into the rack. Since Pilot drivers are paid by the hour, this waiting time does not penalize them. Pilot does not instruct the drivers to obtain fuels from any specific fuel terminals. Drivers may choose any nearby terminals for fuel purchasing, with the lowest cost being the selection criteria, whether or not the fuel is owned by the Pilot company. When a terminal is out of fuel, another nearby terminal can be used.

All "purchasing" processes at the terminals are conducted electronically. The driver used a plastic card with a magnetic strip, similar to a credit card, to purchase the fuel. He entered the codes (e.g., company id, driver id), type of fuel, and amount of fuel, etc., into the computer terminals set up next to the rack. 
When the loading process was complete, a bill of lading (BOL) was automatically generated and sent to the billing company and Pilot. A hard copy was then printed so the driver can keep a copy for his records. The driver also entered this fuel intake information into the on-board system (before leaving the terminal), which was immediately transmitted to Pilot's headquarters via PeopleNet.

\section{Offloading operations}

Before offloading the fuel at the destination gas stations, the drivers used a long stick to measure the depth of the existing fuel in the underground storage tank. This is a common practice, according to the drivers. It serves as an added precaution to ensure the storage tank can hold the amount that will be offloaded into it. Another purpose of using the long stick is to verify whether or not there is water in the tank (e.g., due to leaking or other reason). If water is present, the end of that stick will turn red to provide a warning signal.

The offloading process required some time to complete because it is basically a gravity-based flow and is being conducted one compartment at a time at the speed of approximately $400 \mathrm{gpm}$. The drivers would ensure fuel was completely drained out from the line to the underground storage tank before they proceeded to offload to another compartment.

After shutting off all the valves and securely locking all equipment, the drivers would enter the store (Pilot gas stations) to obtain a signature on the BOL from the store managers. The drivers would also enter their delivery information into kiosks (computer terminals) inside the stores. Information entered by the drivers includes time, date, who made the delivery, how much fuel was delivered, what type of fuel, etc. A printed report of the fuel delivery with bar codes would then be generated from the kiosk. Printed copies of the BOLs, from both fuel intake and delivery, as well as the bar code report were then faxed back to Pilot headquarters before the drivers left the Pilot stores. The abovementioned information was also submitted to Pilot headquarters again via PeopleNet after the drivers returned to their cab.

\section{Comments from the Evidential Reasoning Team}

Based on what was learned from the visits with the drivers, the Evidential Reasoning Team recognized that the entire delivery process was not as straightforward as what the project team had originally expected. The flexibility of delivery schedule and locations could add complications in the geo-fencing construction, evidential reasoning process, and exceptions detections. Therefore, it was recommended that a meeting with Pilot dispatchers to learn the fuel delivery operation and process, as well as to request access to their routes and communication information, would be beneficial to the project team. The team pointed out that the availability of historical BOL info would enable the construction of realistic geofencing model and the "rules" building for detecting exceptions.

\subsubsection{Meeting with Pilot management team at Knoxville Headquarters}

A group of project team members held a meeting with the Transportation Operations Manager and the Central Dispatch Manager at the Pilot headquarter in Knoxville, Tennessee, in 2010. Discussions during the meeting focused mainly on the operations of dispatching and tanker driver schedules. Highlights on information gathered from that meeting are briefly summarized in this section.

\section{Dispatching}

The team learned that a dispatch plan is set by the dispatcher for the following shift based on information obtained from the previous 6-10 hours. Because of this, it is well understood that changes to the plan are likely. Roughly speaking, changes to the daily dispatches are made about $35 \%$ of the time. There are two 
main goals that a dispatch plan is meant to achieve: (1) obtain the lowest priced fuel that is available and (2) get fuel to gas stations (Pilot stores) before they run out of fuel. In order to achieving these goals, dispatchers use information from an in-house inventory system, which provides sales projections at the different Pilot stores, as well as a web-based "feeder-root system," which tracks the inventory of those stores. Simple math is used by the dispatchers to estimate the number of loads (deliveries) that each store needs.

Various reasons as to why a driver's schedule may change were also discussed. These include such situations as when competitors pull out of certain business deals, Pilot could agree to sell gas to those waiting customers, or when the price of fuel increases at other competitors' stations, Pilot stations (being cheaper) would attract more customers, thus increasing the store demand and requiring more fuel deliveries. Also, if a fuel storage tank at a Pilot store has a manifold valve that does not function properly, this could cause the fuel to flow through the tanks in an abnormal fashion, the end result being that storage tank capacities are significantly reduced. More tanker deliveries must be added in order to counteract this issue. This creates a problem for the dispatcher. There might be other accidents and/or personnel issues that would require reallocation of deliveries or reassignment of drivers. Logistic issues could also be a reason.

At Pilot, most drivers work in a 12 hour shift, and the shift schedule is adjusted to each driver's personal schedule. Within those 12 hours, 10 hours are allocated for transit time (on-duty driving or other "work"), and 2 hours are allocated to non-transit activities (e.g., loading and offloading gas, lunch break). For loading and offloading fuel, typically $30 \mathrm{~min}$ is allocated for completing this job, although the actual time could vary from 15 to more than $30 \mathrm{~min}$. An example of a long offloading time could be a situation in which a vehicle is parked over the storage tank access area but the driver cannot be tracked down quickly to move that vehicle. The tanker driver would have to wait until the access area is clear in order to offload the fuel.

\section{Communications}

For communications, Pilot subscribes to the PeopleNet, which is a telematics system that provides communications as well as GPS tracking over time. The information communicated thru the PeopleNet can be recorded and stored for later use. Driver activities, such as loading, offloading, or lunch breaks, can be logged thru the on-board device. PeopleNet does not record these activities specifically; Pilot asks their drivers to put a note in the record only if a delay lasts $45 \mathrm{~min}$ or more. PeopleNet only records where the driver actually went, not whether there were changes in delivery locations. Pilot has started to record information on where the driver was supposed to go (i.e., location originally assigned by the dispatcher), and where she/he actually went.

Under the current setup, when a driver travels for more than 5 min, PeopleNet automatically logs that the driver into the on-duty driving mode. Hours are automatically tracked by the on-board PeopleNet system for hours-of-service requirement. Pilot can access data from PeopleNet through a web tool; currently PeopleNet retains the data for a 6 month period. The older data is stored on a main server.

\section{Staffing and Operations}

Pilot typically staffs and equips their fuel delivery tanker fleet to handle $80-90 \%$ of sales. When they need more deliveries, they will use for-hire tankers and drivers. Pilot's planning calls for the utilization of for-hire work in the remainder of its deliveries in order to minimize possibility of lay-offs during down time. The Central Dispatcher Office (in Knoxville) handles fuel dispatching activities for 215 Pilot stores. The Knoxville-based Pilot tanker fleet (about 13-15 trucks) serves approximately 100 Pilot stores within a 100 mile radius. Within the Knoxville region, there are about 45 Pilot convenience stores. Stores outside 
the 100 mile region are served by other Pilot tanker fleets in other locations. For stores outside of what Central Dispatch serves, Pilot uses lead drivers to handle the tasks. Pilot drivers are not assigned to specific stores but typically work in specific regions. These regions are known as "bands," and there are enough Pilot bands to service the entire United States. Typically, driver assignments must also follow a certain "turn-time.” Drivers are given tasks where they begin at an origin, deliver to a destination, and then return to the origin. Turn-times refer to the average amount of time it takes to complete this cycle. Depending on the distances between origin and destination, turn-times vary from 3 to 9 hours. Typically, Pilot tankers do not go long enough distances to require an overnight stop; their tankers are "day cabs" which do not include sleeping space or other amenities. Overnight stays would serve to increase costs due to the miscellaneous expenses involved.

It was confirmed that the federal fuel tax is assessed at the rack (fuel terminals). At the time the fuel is received from the fuel terminal, the Pilot tanker truck drivers must specify the intended fuel delivery state (for federal and local fuel tax purpose). If the Pilot dispatcher reroutes the tanker to another state, additional paperwork must be made so that the appropriate taxes are assessed. Instances of fuel theft that Pilot experienced were also discussed. In one situation, the driver was selling a portion of his fuel to a competitor and pocketing the money. In other instances, the driver might park behind a trucking business or a large store, such as a K-Mart, and pump his/her fuel to an enclosed trailer. In the latter instances, the theft was detected through the GPS tracking system. Still, it was emphasized that the drivers were barely considered "off-course" in this situation. In this case, perhaps the sensor solution might have helped Pilot to detect the theft activity much sooner. In addition to delivering fuel to its gas stations, Pilot also delivers fuel wholesale to other gas stations. Pilot also delivers the non-highway-use fuel (dyed diesel) for agricultural and industrial use. This information implies that tax evasions are possible within Pilot's operations.

\subsubsection{Truck Travel Behavior Data (PeopleNet Fleet Manger Reports)}

In order to study travel patterns of tank trucks during their fuel delivery operations, it was necessary for the team to examine historical and other travel data from Pilot. To protect potentially sensitive or proprietary information related to Pilot operations, with the approval of UT-Battelle Legal Department, members of the Evidential Reasoning Team entered a nondisclosure agreement with the Pilot to receive Pilot's PeopleNet data. With this agreement fully executed on October 28, 2010, the Evidential Reasoning Team was granted access to Pilot’s PeopleNet Fleet Manger system.

Many preconfigured custom reports are provided by PeopleNet to their subscribers for managing their fleet operations. Users can select their fleet of interests and specify a day, or a range of days (up to 7 days), to obtain reports. Reports that are relevant to this research study, specifically, are those related to GPS locations and travel activities (circled in red in Fig. 57).

Three GPS location reports were available, including GPS Location Report, GPS Location by Hour Report, and GPS Overview Report. Only the GPS Location by Hour Report (Fig. 58) provides specific latitude-longitude (lat-long) location information for the vehicles at the hourly "response" time (the time it was "pinged" by PeopleNet). The more detailed GPS Location Report only shows landmark names indicating where the vehicles are located at the time. The landmark name is clearly easier and more meaningful for the fleet managers to track their vehicle locations. This convention, however, created some added complications for the Evidential Reasoning Team. In order to use the information for the geospatial analysis required to develop the tracking and geo-fencing tool, these location names (landmarks) had to be translated back to lat-long values, requiring additional effort for geo-coding and conversions. 


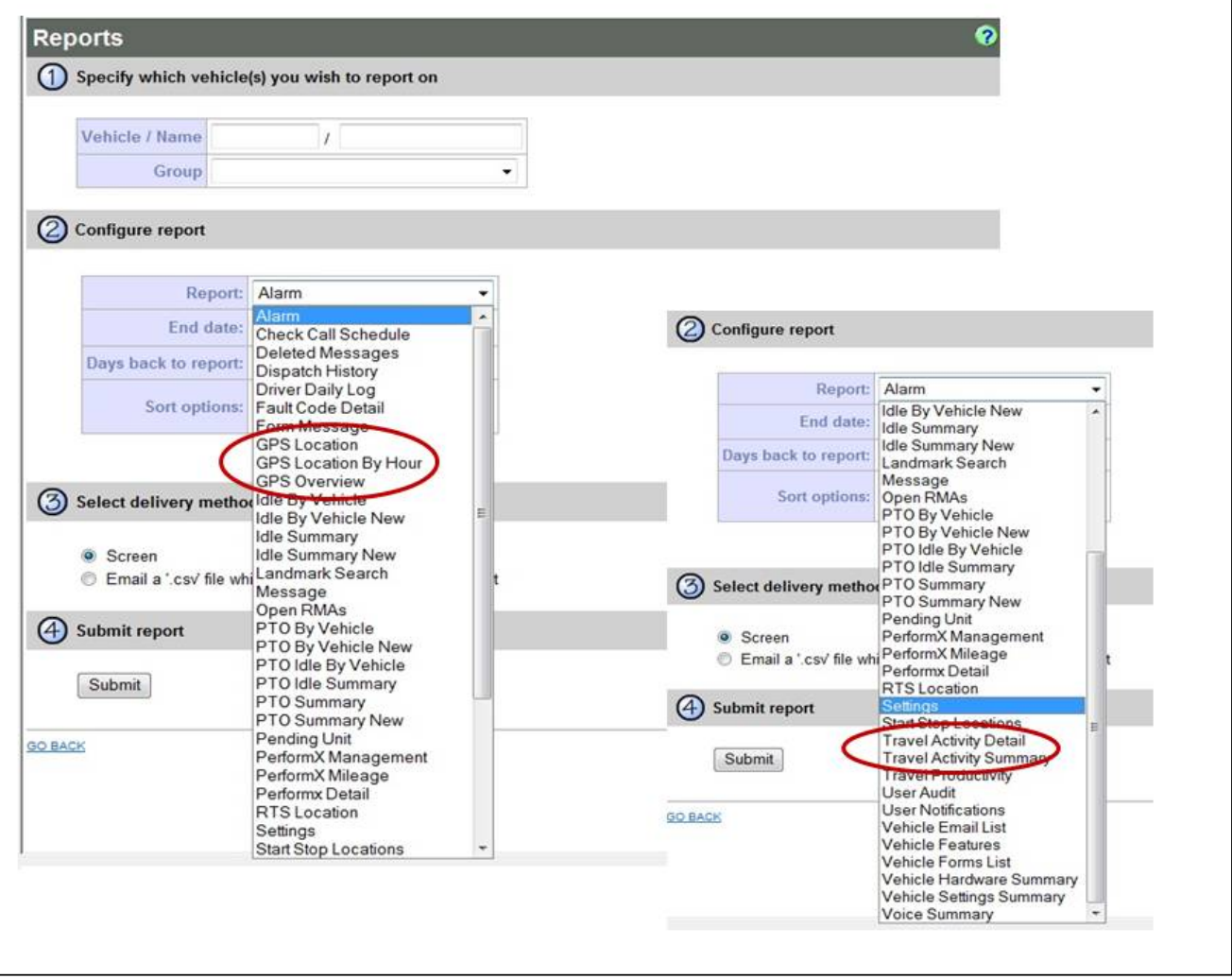

Fig. 57. Types of reports available from PeopleNet Fleet Manager.

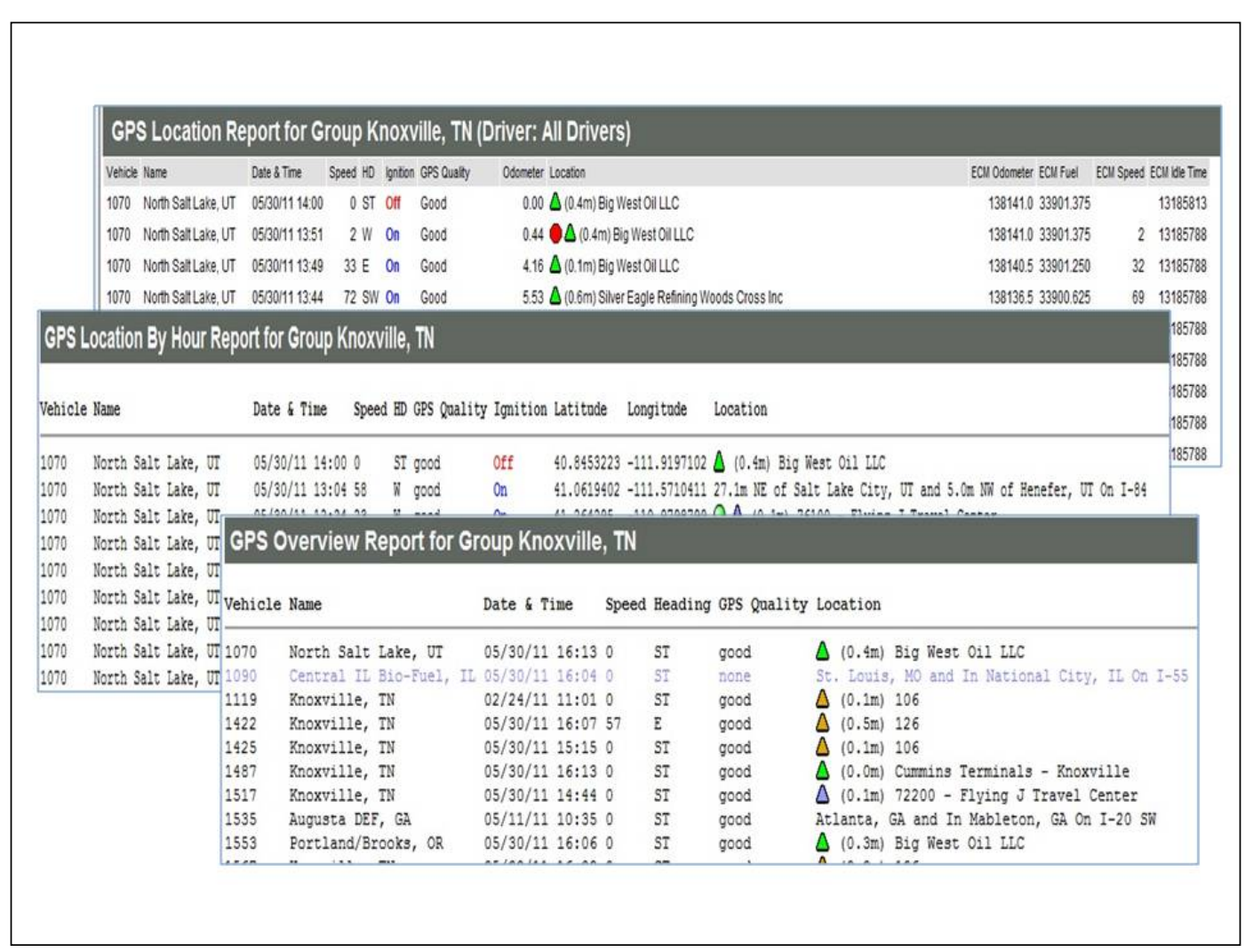

Fig. 58. Examples of GPS location reports available from PeopleNet. 
In addition to viewing the report on screen, PeopleNet allows its users to e-mail the report to themselves as a CSV (comma separated value) file. This allows the users to conduct their own analyses using the downloaded data. For this project, the limit of 7 days (for creating a report) posed a slight inconvenience for the team; to obtain an entire year of historical data from one report, the "e-mail and save file" process had to be repeated 52 times.

The Travel Activity Reports (detail and summary, see Fig. 59) provide a slightly different organization of the same information presented in the GPS Location Reports. Instead of sorting through all records of the GPS location report data, since these activity reports contain only records related to stops, it allows the Evidential Reasoning Team to examine the stop durations by type of stops more efficiently. Similarly, the "e-mail and save file” process was conducted 52 times to obtain a year of historical data.

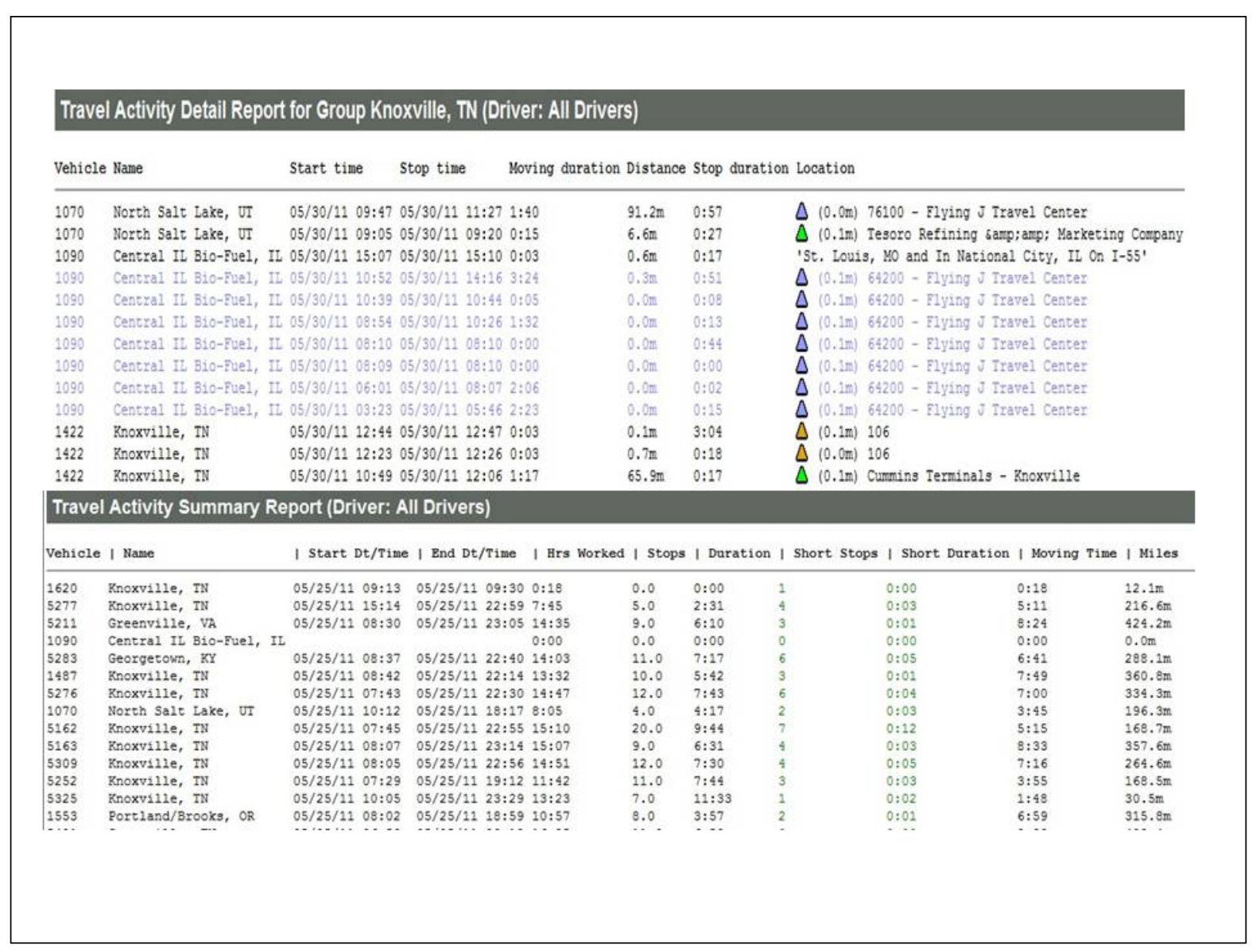

Fig. 59. Examples of Travel Activity reports.

\subsubsection{Service Station, Terminals, and Wholesale Customer Locations}

Additional web searches were conducted to obtain location data to further supplement information collected based on PeopleNet GPS reports. Types of stops were categorized by the Evidential Reasoning Team, for the purpose of this research study, based on characteristics of these stop locations. Specifically, using information collected from the Pilot’s PeopleNet travel activity report (Sect. 5.2.1), stops were

grouped into three classifications, including terminals (fueling location or origin of a delivery supply chain), Pilot-owned stations (offloading location or destination of a delivery supply chain), and wholesale or other customers (other offloading location or destination). As mentioned previously, landmark names 
were provided by the PeopleNet reports, instead of lat-long coordinates needed for geospatial study use. Geospatial location of these stops were therefore identified through the use of several information sources, including Pilot company website (specifically for locations of Pilot and Flying J stations), PeopleNet location identification tool (i.e., Landmark Search), as well as the use of a Google Earth application.

The map shown in Fig. 60 displays all Pilot-owned service stations (including Pilot and Flying J). Not surprisingly, most of these service stations are located along the interstate highways (highway network not displayed for stations within Canadian border). In addition to Pilot Travel Centers and convenience stores and Flying J fuel stations, Pilot-Flying J also owns several other service stations throughout the United States (shown in yellow triangles).

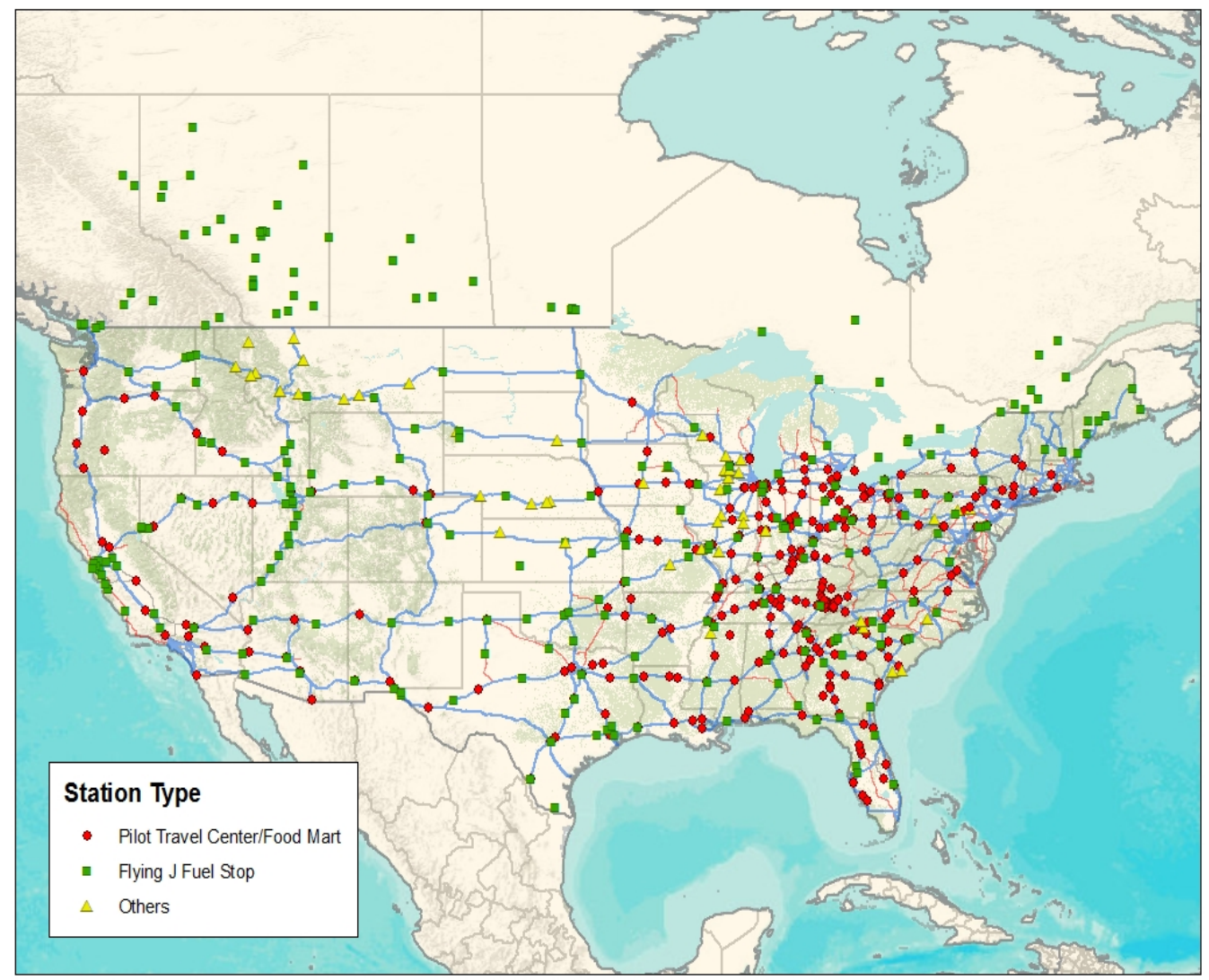

Fig. 60. Service stations owned by Pilot-Flying J, LLC.

The focus of this study was to learn tanker truck delivery operations and fueling processes, with Pilot's participation and assistance, so that realistic tools (geo-fencing and evidential reasoning) could be developed to detect potential FTE as early as possible. To limit the scope for a POC study, data on Pilot operations around the Knoxville, Tennessee, area (where Pilot Headquarters is located) was used. Examples of the three types of stops, that is, service stations, terminals, and wholesale/other customers, are presented in Figs. 61, 62, and 63. 


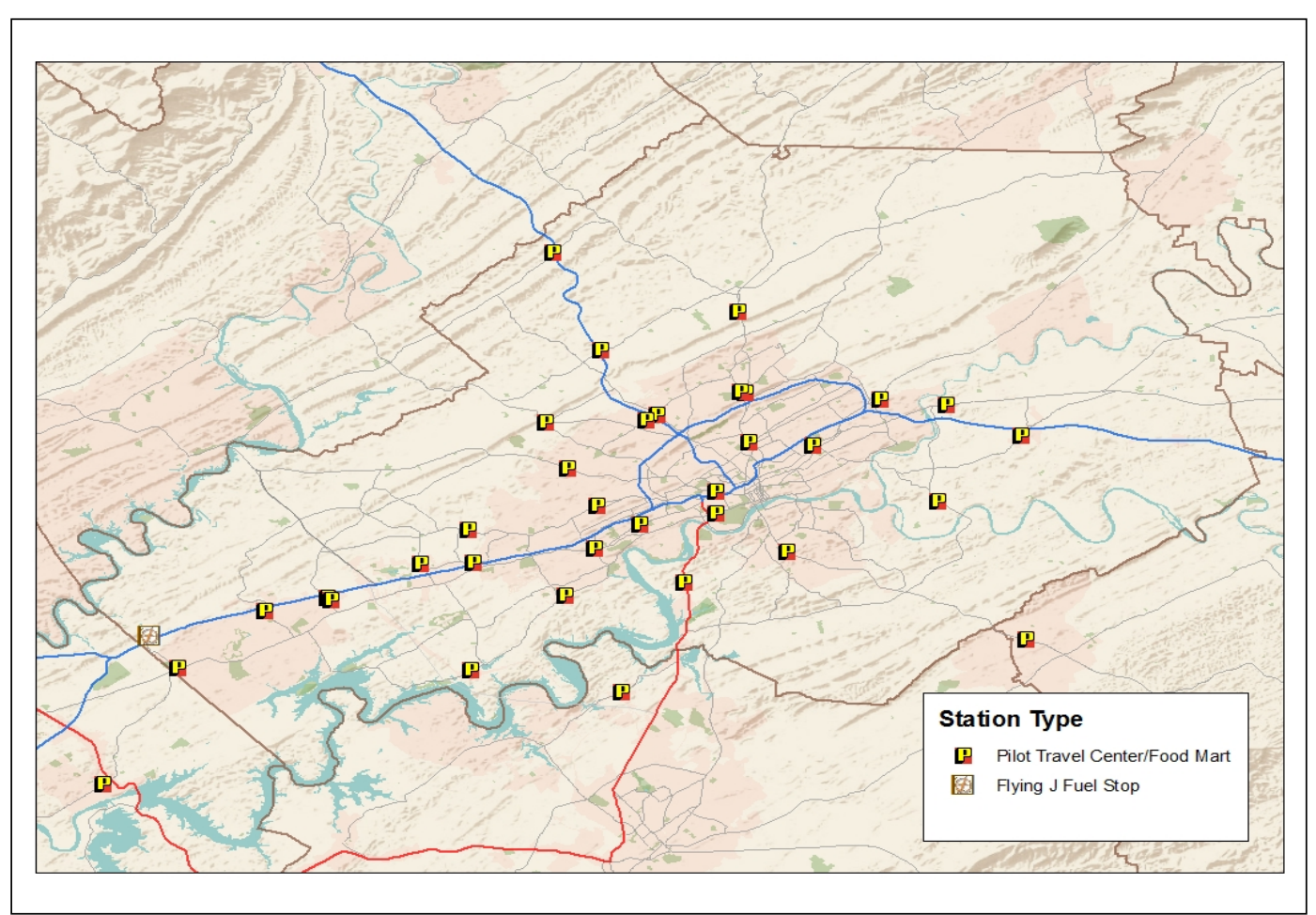

Fig. 61. Pilot and Flying $J$ service stations around the Knoxville, Tennessee, region.

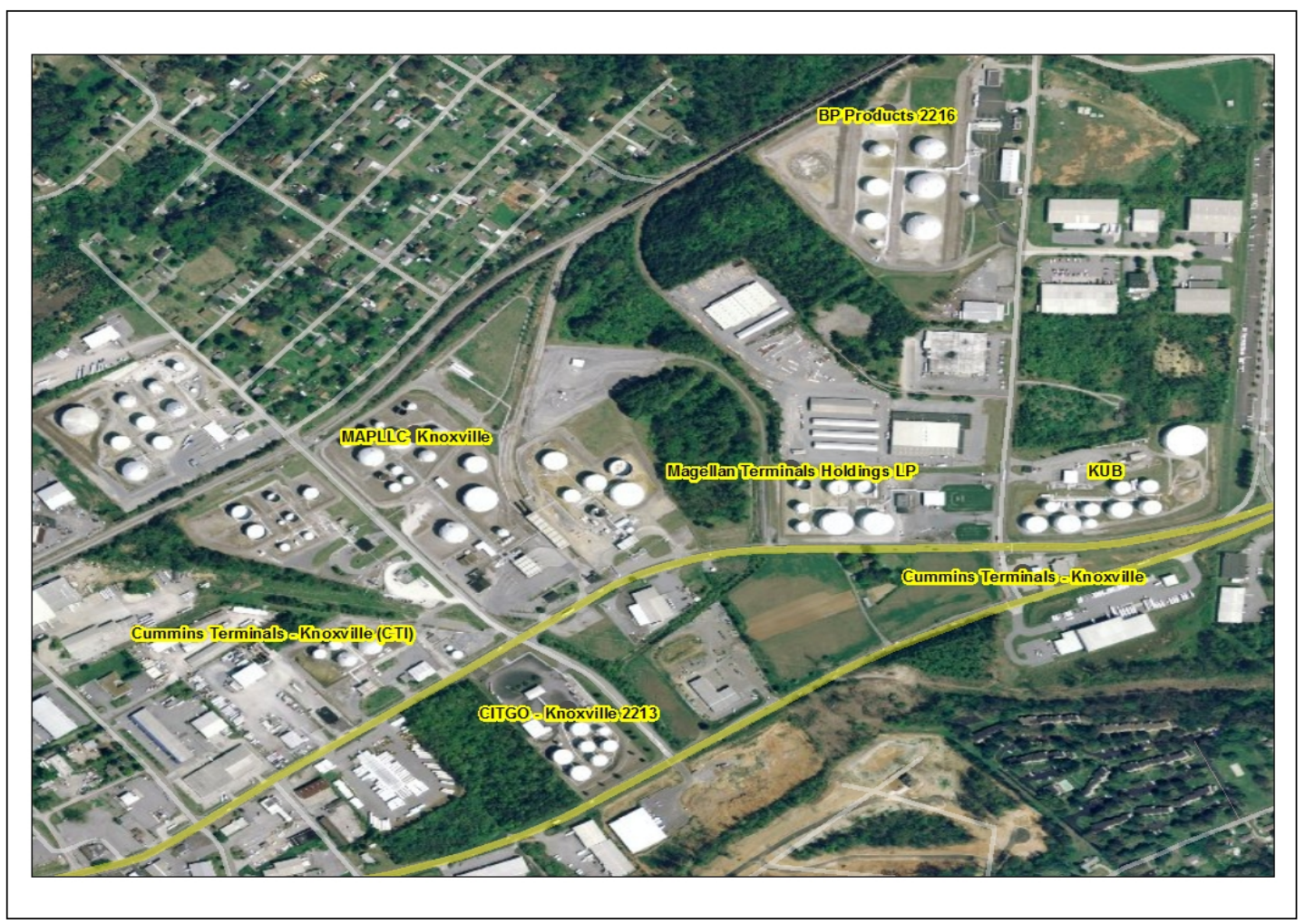

Fig. 62. Terminals around the Knoxville, Tennessee, region. 


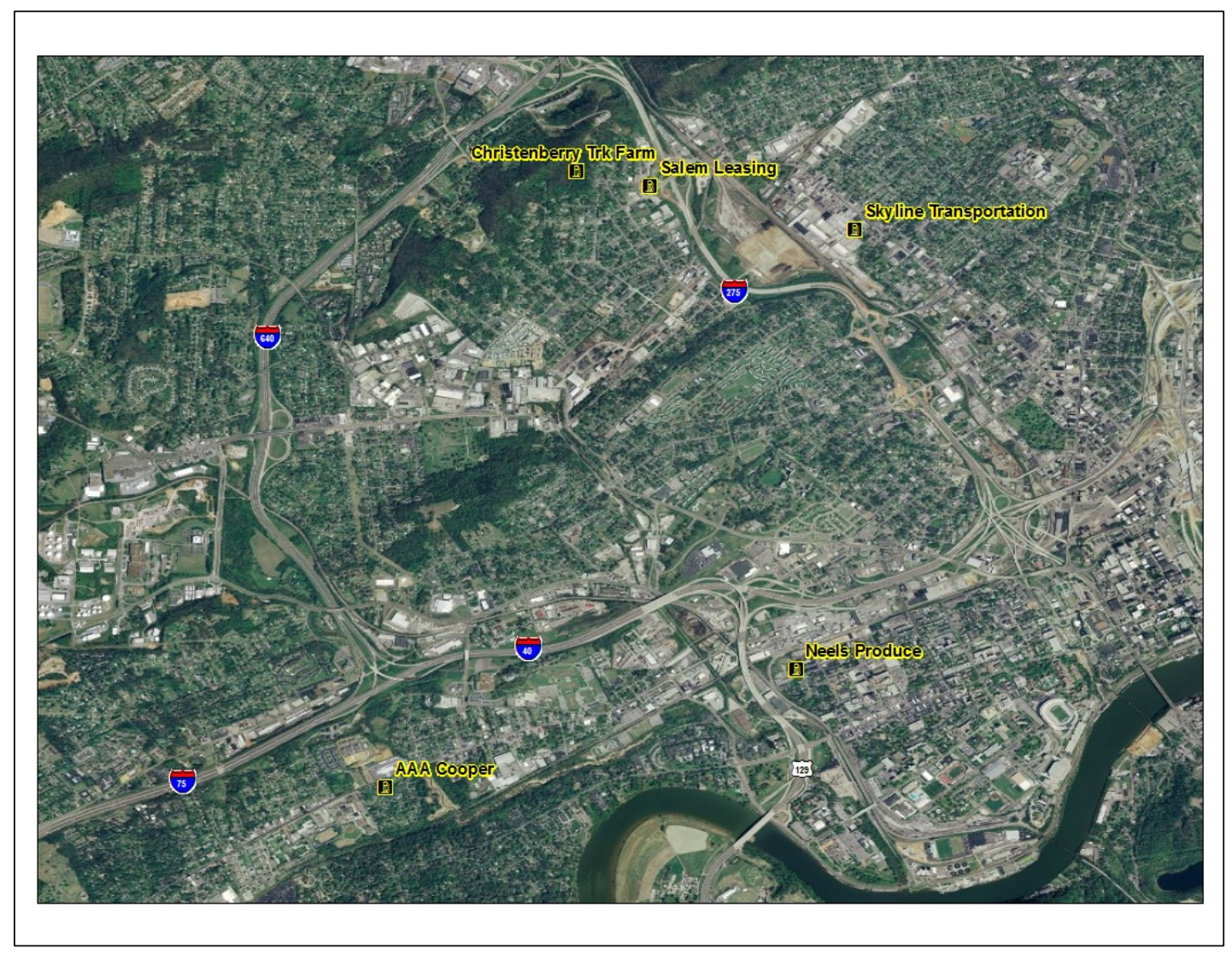

Fig. 63. Examples of wholesalers and other customers served by Pilot tankers.

\subsection{ANALYSIS OF TRUCK TRAVEL BEHAVIOR DATA}

\subsubsection{Duration of Stops}

As discussed previously, the reports downloaded from the PeopleNet Fleet Manager allowed databases of GPS location and travel activity data on Pilot tanker truck for the calendar year 2010 to be compiled and analyzed by the Evidential Reasoning Team. Specifically, the data was used in estimating the length of stop for loading and off-loading activities. This allowed the team to establish a stop-length (duration) distribution for each fueling activity (fueling and off-loading). This information could then be used to construct rules and weights (likelihood, probability, or belief) for determining unusual or suspicious behavior associated with fuel loading and offloading activities.

The length of stop estimates are calculated separately for each type of stops as discussed in the previous section; they are terminal, Pilot-owned stations, and wholesale and other customers. These types were classified using the stop location names given in the PeopleNet reports. Stop location names that did not contain specific station names (or store IDs), terminal names, or other company-like names were excluded from the duration analysis. For example, locations with names such as "in Knoxville, Tennessee and 3.7m SE of Karns, TN near I-40” and “69.7m N of Knoxville, TN and in N Corbin, KY on I-75” were clearly traffic stops. Thus, these records were excluded from the analysis.

It is also possible that a specific landmark name could be shown in the activity report as the stop location when the truck might be stopping for other activities at a location that happened to be close to the 
landmark (e.g., Pilot headquarters). Thus, records with stop location names containing "HQ” are also excluded from the analysis dataset.

Note that although the GPS location data could be used to "track" the tanker truck movements, there is no specific information on any specific "routes" used, nor whether a delivery location was for a designated store on the dispatcher's original schedule (i.e., the dispatch plan) or a new/revised delivery location based on a last-minute change. Furthermore, there was no information on why a tanker stopped at a particular location, or what business being conducted at such a stop was. The activity, for the purpose of estimating the stop-length distributions, was assumed to be fuel loading when the stop was at the Knoxville terminal area and was assumed to be off loading when the stop was located at a Pilot or Flying J service station or a wholesale/customer location.

No attempt was made to sort through the detailed records to add up individual durations associated with the same stop into one accumulated total length for any given loading or offloading stop. For example, when arriving at a terminal, the tanker truck could be joining a queue with a few stop-and-go movements before finally reaching the position at the rack for fueling. Each of these stop-and-go segments would result in a short-duration record, while the record that captures the actual fuel loading would have a longer duration associated with it. Since the focus of this study is more toward the significantly longer durations (given that it is at a legitimate location) and for a POC research, additional efforts to clean up the dataset (related to a specifically company's operation) would not be cost beneficial to the project as a whole. Thus, one should bear this in mind when reviewing the distributions shown in Figures 64, 65, and 66, the durations shown are not necessarily indicative of the fuel-hauling industry as a whole.

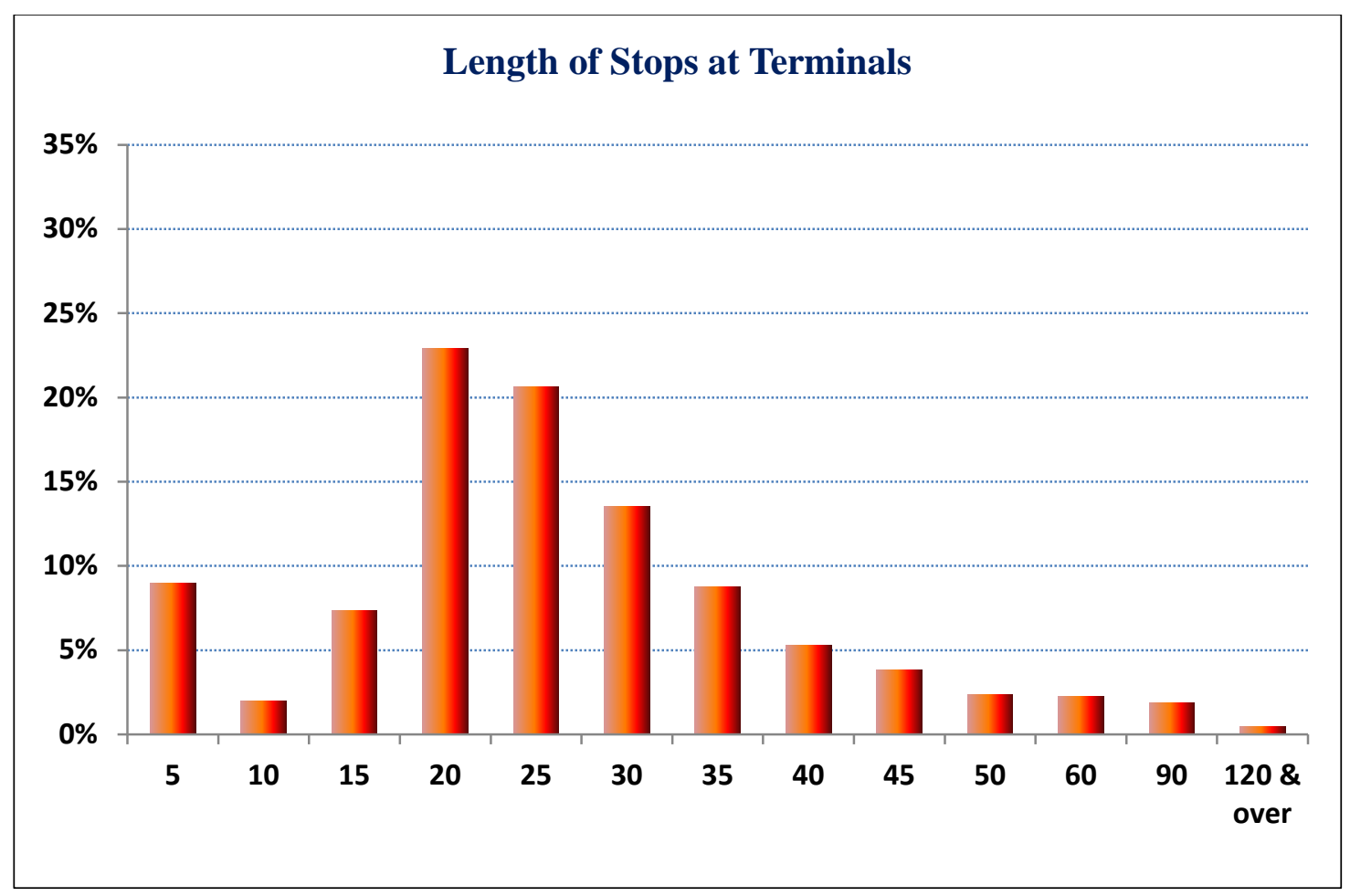

Fig. 64. Distribution of stop durations at the terminals (in minutes). 


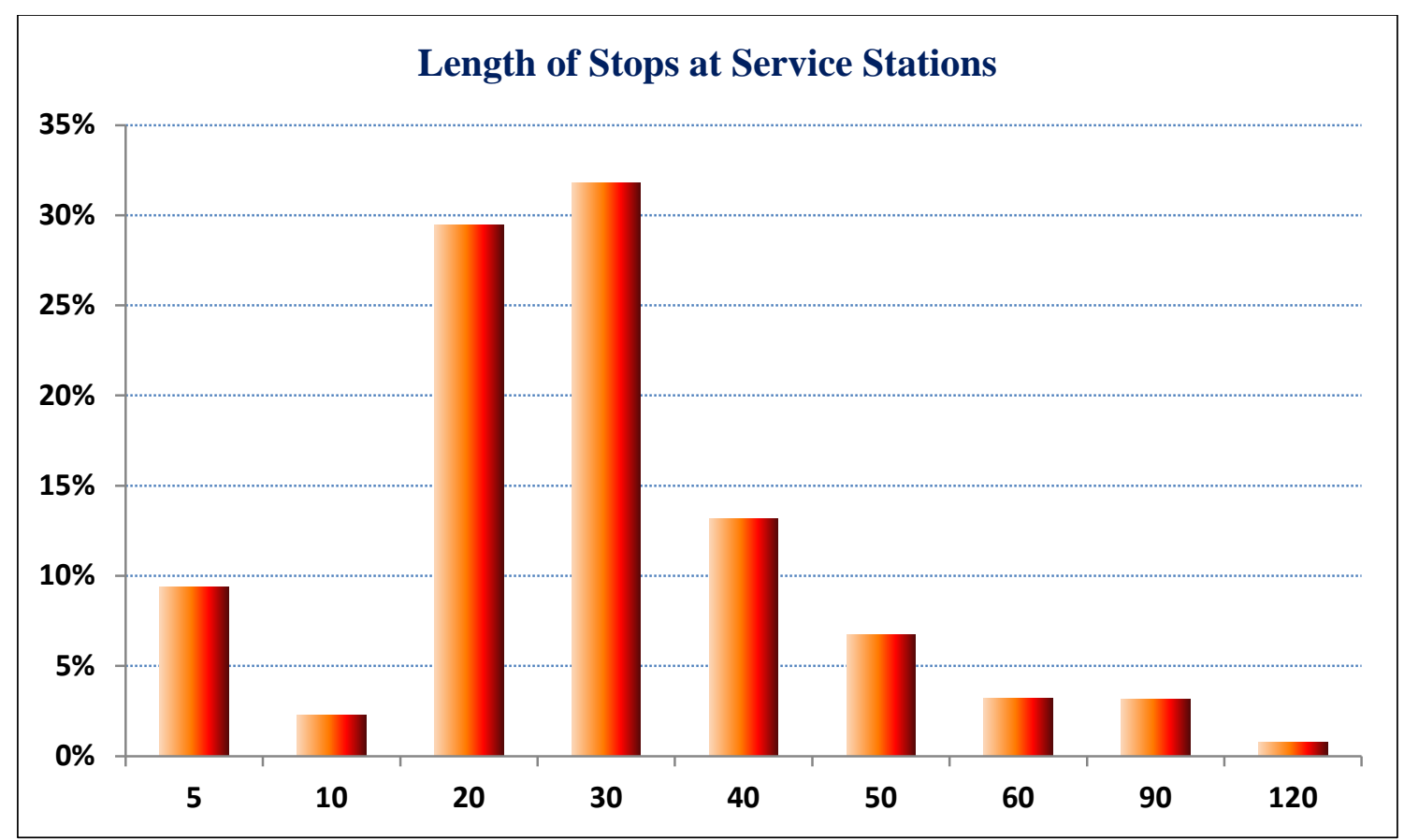

Fig. 65. Distribution of stop durations at gas stations (in minutes).

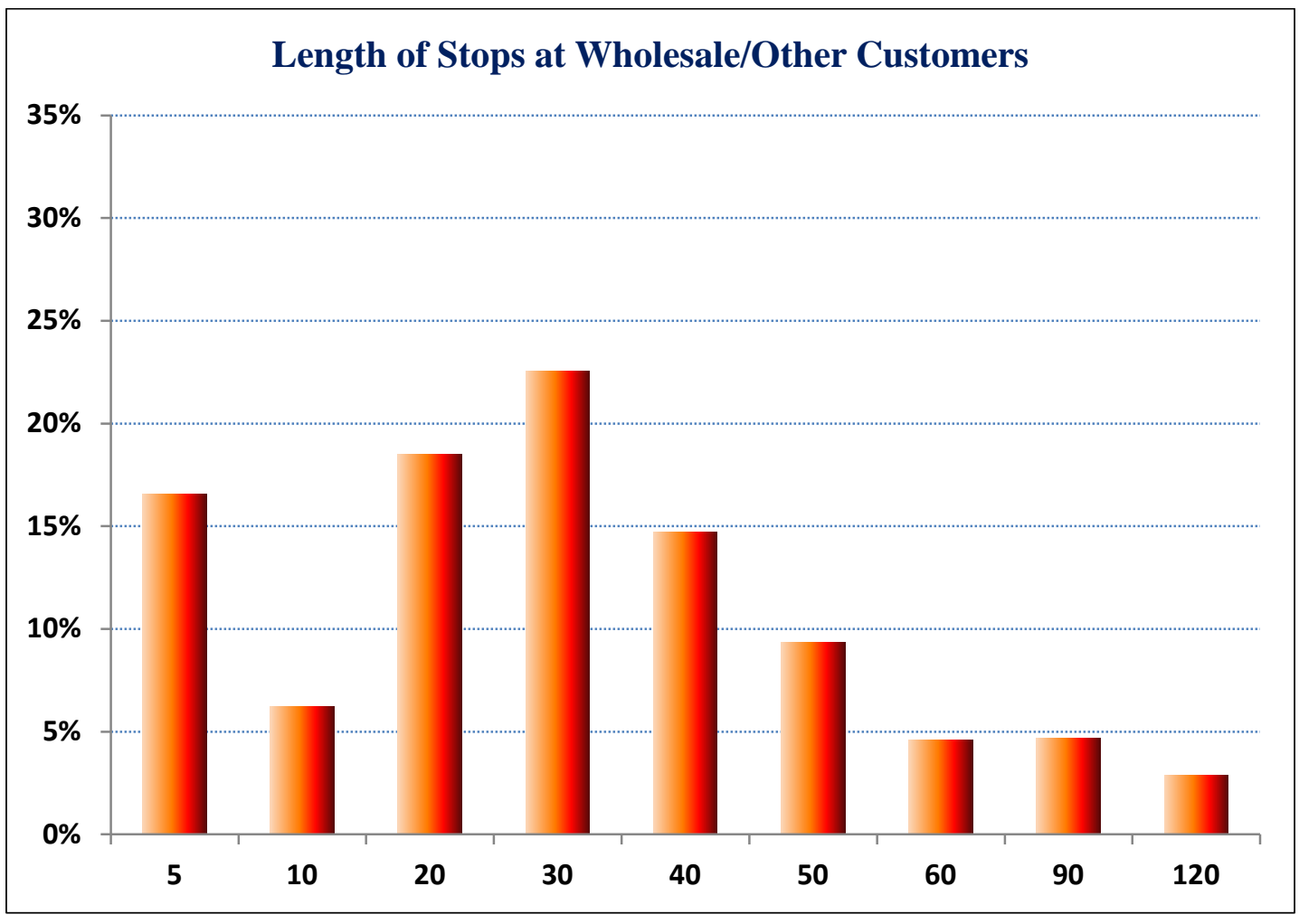

Fig. 66. Distribution of stop durations at other locations, including wholesale customers (in minutes). 


\subsubsection{Travel Routes}

In addition to providing an opportunity to examine stop durations, data gathered from the PeopleNet Fleet Manager reports were also used to study typical routes for fuel delivery operations (based on GPS information). The route patterns of Pilot tanker trucks were used to generate simulation cases discussed later in this section. Realistically, one cannot expect a fixed route to always be used when delivering fuel between a certain pair of origin (terminal) and destination (service station) locations. For example, as shown in Fig. 67, there are three routes which are most likely to be taken to travel from a fuel terminal located in Knoxville to a Pilot service center off the Interstate 40. Here route A is via Interstate 40, route $B$ is on the Interstate 640 bypass, and route $C$ is on a state highway (Middlebrook Pike) in the Knoxville area. By examining the GPS data over time and with the aid of the team's understanding of local travel routes and traffic conditions, basic rules for route selections could be built and used in developing geofencing methodologies.

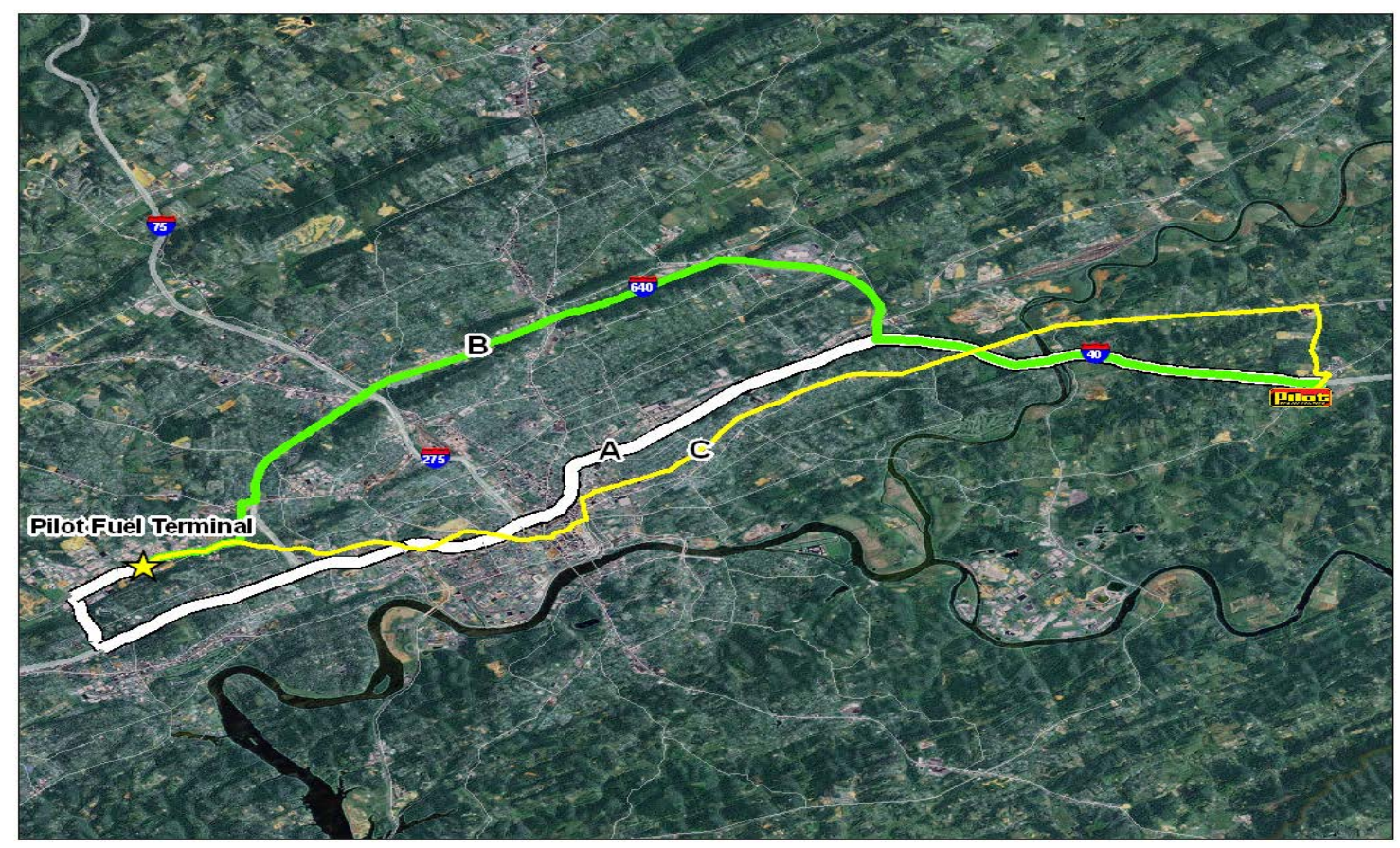

Fig. 67. Example of multiple route choices between a specific terminal and service station pair in Knoxville, Tennessee.

\subsection{GEO-FENCING APPLICATION}

\subsubsection{Methodologies to Detect Out-of-Fence Travel Behavior}

Geo-fencing is a relatively new technology advancement of telemetric hardware and software. This concept refers to the practice of limiting human or equipment movements to a specific geographic location by tracking their whereabouts via GPS technology. For the purpose of this effort, geo-fencing is used to make sure certain mobile equipment or people stay within the boundaries specified by the monitoring entity. The technology allows users of the system to draw zones around places of work, customer sites, secure areas, and/or paths along a route. These geo-fences, when crossed by an equipped vehicle or person, can trigger a warning to the user or operator via text message or e-mail. In extreme circumstances, the zones can be linked to immobilization equipment within a vehicle and disable the engine. 
Geo-fencing boundaries generated based on the predetermined routes or anticipated paths are considered static geo-fencing boundaries. In common fuel delivery tanker dispatching practice, the dispatcher only instructs the driver where to deliver the fuel. The driver has the responsibility to find the fuel terminal with the lowest fuel price at the time of refueling the tanker, and the driver also has discretion regarding which route the tanker will take to reach the refueling service station. The tanker driver might also need to deviate from his originally planned route due to unexpected circumstances such as traffic accidents or congestion. Static geo-fencing boundaries do not consider the above-mentioned dynamic situations.

To compensate for the static nature inherent in the pre-determined the delivery route, the distance between the current position of the fuel tanker and the location of the delivery service station was calculated and compared to previous calculated distances over time. Great-circle distance was used in these calculations as the distance measurement.

The great-circle distance is the shortest distance between any two points on the surface of a sphere measured along a path on the surface of the sphere (as opposed to going through the sphere's interior). Because spherical geometry is rather different from ordinary Euclidean geometry, the equations for distance take on a different form. The distance between two points in Euclidean space is the length of a straight line from one point to the other. On the sphere, however, there are no straight lines. In nonEuclidean geometry, straight lines are replaced with geodesics. Geodesics on the sphere are the great circles (circles on the sphere whose centers are coincident with the center of the sphere). Because the earth is nearly spherical, equations for great-circle distance can be used to roughly calculate the shortest distance between points on the surface of the Earth (as the crow flies), and so have applications in navigation. Based on the cumulative distance progression information, one can easily judge the fuel tanker is getting closer to or moving away from the delivery service station. Although alternate methods of distance calculation may have been sufficient for the limited testing area of the Transportation Research Center (TRC), this methodology was selected to be suitable for real-world situations.

\subsubsection{TRC Roadway Network}

Due to safety and liability concerns, actual on-road testing of an instrumented tanker truck (with sensors and communication devices installed) was not possible. Instead, all field tests were conducted at the TRC's closed test site. TRC is an independent automotive proving ground located on approximately 4,500 acres of land in East Liberty, Ohio, approximately 40 miles northwest of Columbus. TRC Inc. conducts programs designed to test for safety, energy, fuel economy, emissions, durability, noise, crash, crash simulation, and performance. TRC test trucks, buses, recreational vehicles, motorcycles, electric vehicles, passenger cars, and components.

There are several test tracks spread throughout the TRC proving grounds. Only certain testing areas and routes were used in the field testing. After preliminary study and consultation with TRC personnel, four locations within the TRC facilities were selected as the refueling and fuel delivery locations (Fig. 68). These four locations are Building C, Fuel Plaza, Southeastern Vehicle Dynamics Area (SE VDA), and Winding Road Course (WRC) Pad.

Although testing was performed at the test track rather than in a real-world situation, the testing of the hardware components (i.e., sensors and communication devices) was not expected to be impacted dramatically. As a result of this change, however, many rules and algorithms which were developed based on real-world Pilot data for the geo-fencing and evidential reasoning components of this study were no longer directly applicable to data collected at this controlled testing location. For example, the rules regarding distribution of stop durations and alternative routes could not be applied to scenarios tested at the TRC site. 


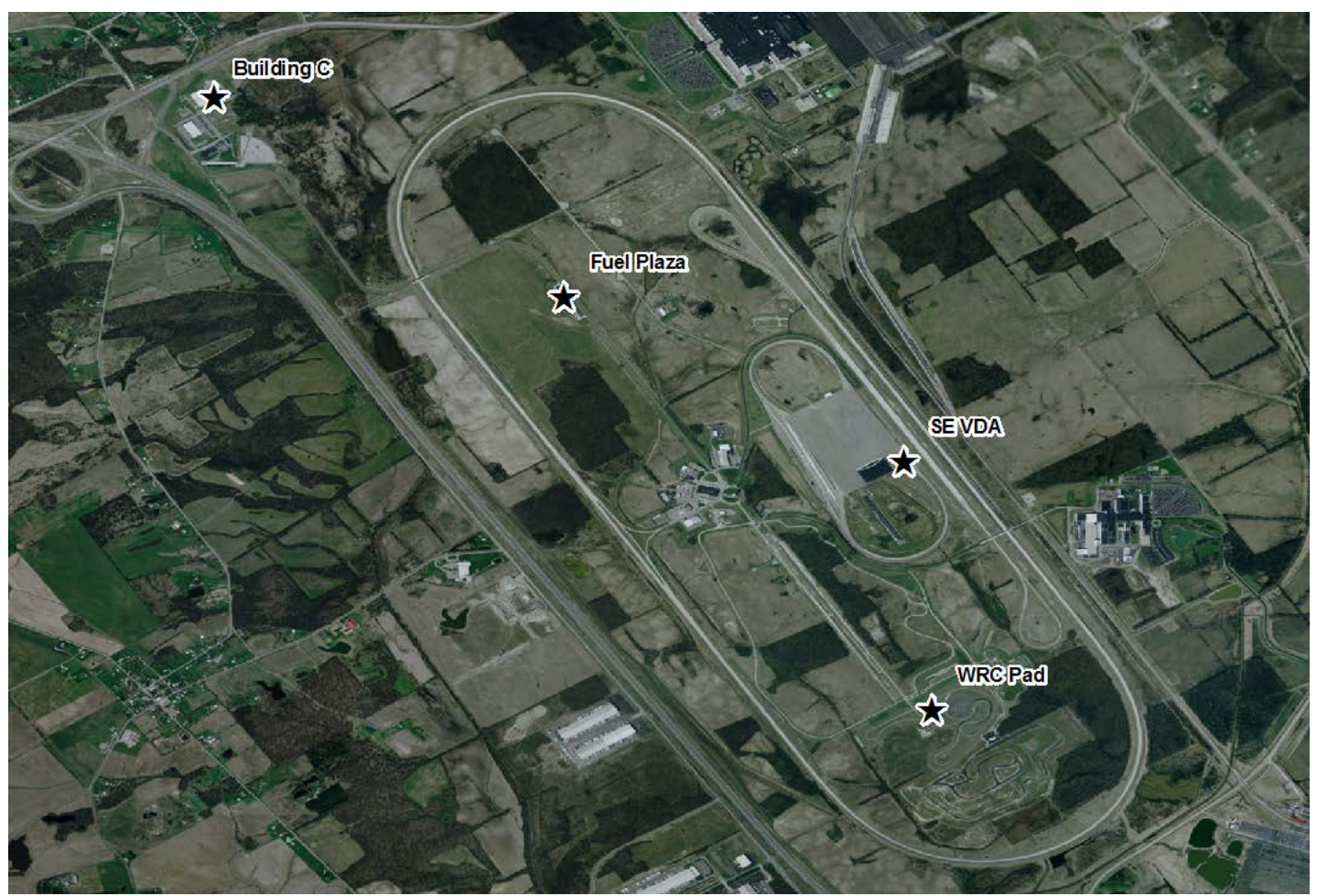

Fig. 68. Selected simulated refuel and delivery locations in TRC.

In addition, the new setting required a routable roadway network to be built for the TRC site so that routing of the delivery truck could be conducted and a geo-fencing buffer zone for each test route could be determined. Figure 69 shows the entire digitized roadway network in the TRC test site.

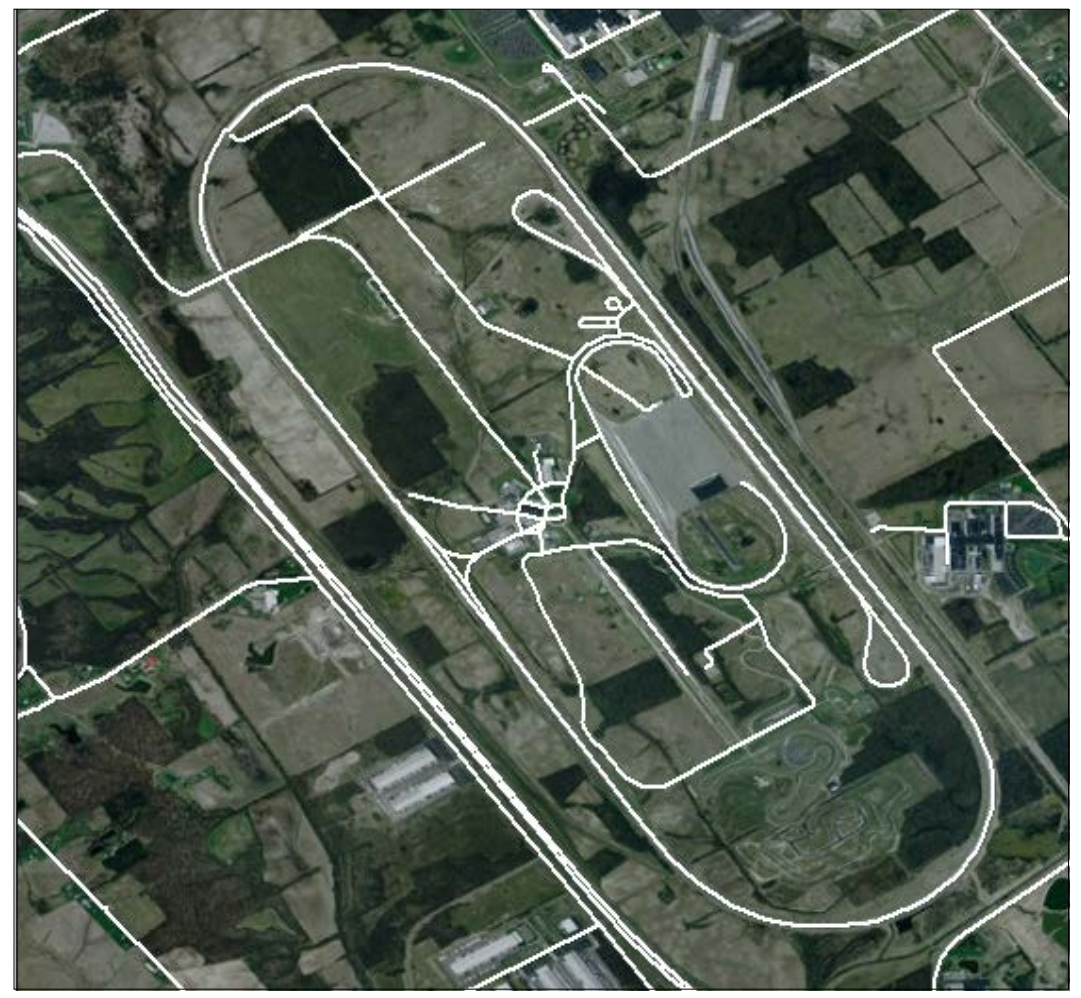

Fig. 69. Digitized roadway network around the TRC test site. 


\subsubsection{Location Tracking at the TRC}

The location information of the testing fuel tanker (tractor-trailer), in terms of longitude and latitude, was collected every second. The second-by-second location information generated by the on-board GPS unit was stored locally on the on-board data collection device in the cab of the test vehicle. This information was also transmitted, via cell phone communication network, to the evidential reasoning system every 5 min.

Because of the remote nature of the TRC testing location, cell services were spotty at best during the test period. Therefore, the information collected by the FTE project testing data server at 5-min intervals was fragmented. The complete set of data stored in the on-board data collection device was therefore used to fill in the data gaps to facilitate the evidential reasoning process.

The second-by-second location information of the testing fuel tanker enabled the analyst to monitor the routes traveled by the test vehicle. From preliminary location information alone, it proved very challenging to determine and interpret a stop during a fuel delivery trip without any other supplemental data. We decided to use the Valve Open/Valve Close information together with the location information to determine the loading and offloading activities within the overall set of GPS test data.

The location information was used in the following evidential reasoning processes to determine the legitimacy of the fuel tanker's trajectory. The first evidential reasoning location verification process involved geo-fencing. The second evidential reasoning location screening process involved great-circle distance progression between the actual test vehicle location and the scheduled fuel delivery site.

The actual longitude and latitude of the test vehicle was recorded on the on-board device every 1 second. The second-by-second location information was transmitted to the FTE evidential reasoning system every $5 \mathrm{~min}$. That is, at $5 \mathrm{~min}$ intervals, 300 geo-location records were processed to determine the "point within polygon” status immediately after the evidential reasoning system received the latest 5 min data packet. The "point not in polygon" would then trigger a warning to the operator. This information, combined with readings from the sensors, allowed the evidential reasoning algorithm to calculate estimates of the risk levels. When a high risk result is detected in a fielded system, an alert could be sent to dispatcher or enforcement if necessary.

\subsubsection{Geo-fencing Example}

The predetermined route (light yellow) for the nominal scenario (Scenario 1) is depicted in Fig. 70. A $500 \mathrm{ft}$ width geo-fencing boundary (light-blue polygon) along the predetermined route for this scenario is illustrated in the figure. This polygon provides a "buffer zone" or geo-fence so that detection of an outof-bound situation can be flagged.

\subsection{EVIDENTIAL REASONING APPLICATION}

\subsubsection{Overview of Concepts}

The concept of an evidential reasoning tool designed for this study was based on a two-step approach. The first step was to develop a set of heuristic rules that could be used to identify abnormalities, based on information and data collected from Pilot staff/experts, historical operation data (e.g., BOL and schedules) and travel behavior data (e.g., detailed GPS data for delivery routes). These rules provided the basic capability to determine anomalies in the fuel delivery process, including determining out-of-bound buffer for the geo-fencing application. Next, knowledge about uncertainties and operational parameters 


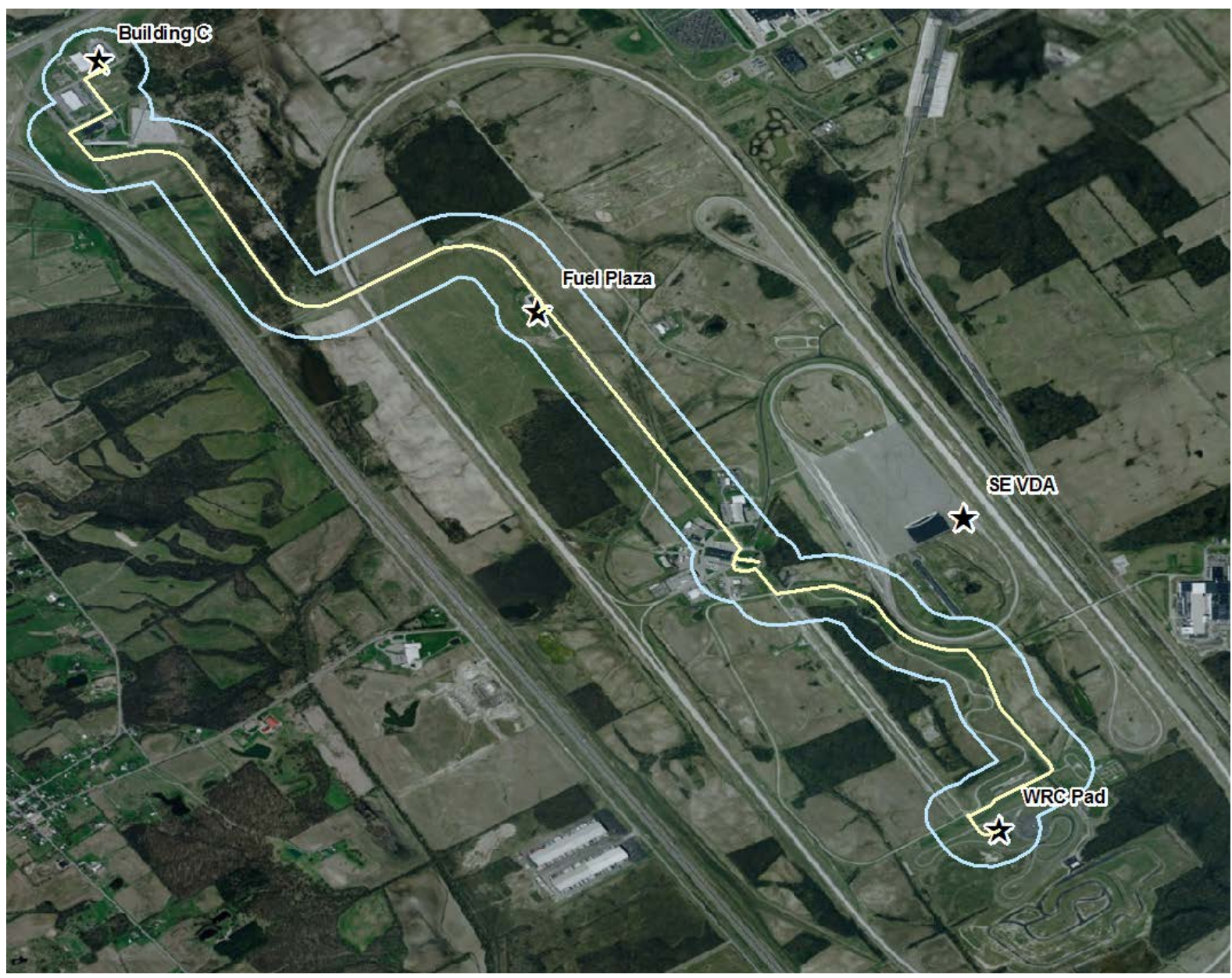

Fig. 70. Predetermined nominal scenario route and geo-fencing boundary.

(e.g., upper/lower bounds of measurement errors from sensor readings and their associated likelihoods or probabilities) was compiled and combined with the basic rules to establish the evidential reasoning tool. This evidential reasoning tool had the capability to determine the likelihood of an anomaly in the fuel delivery system (i.e., the supply-chain from loading to offloading of fuel products) and to alert the dispatcher regarding the status of the fuel shipment. The level of the warning could vary from contacting the driver to notifying local law enforcement authorities.

To take advantage of an existing tool that has already implemented the D-S reasoning technology instead of spending resources to build a model from scratch for this project, the Intelligent Decision System (IDS) which was developed and distributed by the Intelligent Decision Systems Limited ${ }^{*}$ was considered. The IDS integrates D-S theory of evidence with evaluation analysis model to allow one to perform hybrid multiple criteria decision analysis. This Windows-based tool can be accessed free of charge if the number of attributes is small (fewer than 10). Since the number of attributes considered under the current study was fewer than this limit, it was determined that the use of this tool would be a cost-effective option. For this project, attributes to be evaluated included responses from the sensors (valve, hatch, fuel level, and fuel maker) and an indicator generated from the GPS data (e.g., out of geo-fencing or non-scheduled stop).

*See http://www.e-ids.co.uk/ for additional information. 


\subsubsection{Development of Detection Rules}

To begin the development of a reasoning tool, a set of specific detection rules for the intended tool had to be designed and built into the algorithm. The purpose of these detection rules was to enable the developed tool (in this case, a prototype expert system) to automatically perform assessments or evaluations as a subject expert would do. That is, the system took results from an actual condition (e.g., truck stops at location $\mathrm{x}$ ) and compared them to information about the expected or known condition (e.g., the scheduled stop is not $\mathrm{x}$ ). In cases where a significant deviation was identified, warnings or alerts could have been generated to notify a dispatcher or control center operators.

Based on current technology and practicality, it is not possible for a moving vehicle to take on or offload fuel. Therefore, a major prerequisite required for "initiating" the detection rules was that the targeted tanker truck had to be at a stop. Obviously, a truck could make stops at many legitimate locations in addition to stops at terminals for loading or at stations for offloading. Traffic signals and vehicle breakdowns are two examples of likely stops that could occur along a fuel delivery supply chain route.

For situations where a truck was stopped at given location (excluding traffic stops), the following specific actions were used to develop the detection rules under this research study.

(a) Identify whether the stop is scheduled or unscheduled

This involves determining whether the scheduled stop refers to a terminal (for loading) or a destination (for offloading) on the dispatcher-assigned delivery list or is not on the list. It is assumed that whenever changes are made to the delivery location, the dispatcher always knows the most up-to-date destination location. Thus, the scheduled destination would always be where the tanker truck should go to offload its fuel. Using this definition, an unscheduled stop would be a stop that is made at a location different from the scheduled one. Since all activities related to loading or offloading fuels require the truck to be stopped, determining whether the stop is scheduled or unscheduled is therefore an essential first step.

(b) Monitor the vehicle travel route

This is where the GPS data from the telematics system is required. A geo-fencing technique is applied here to determine whether the travel route taken by a tanker is within a reasonable range of deviations from the expected route or scheduled stop location(s). In the TRC testing environment, no route deviations were simulated as the number of alternate routes available in the proving grounds was extremely limited.

(c) Estimate the time length of the stop

From the stop duration distributions discussed previously, rules and/or beliefs could be designed to reflect the possibility (i.e., probability or weight) of the occurrence of longer stops (regardless of whether the stops were scheduled or not). A detection rule which incorporates a review of the stop length could be useful to inform a dispatcher to contact a seemly inactive driver. In other words, a warning or alert could be made for extremely long stops, especially when a dispatcher had not been notified of any incidents. Under the limitation of TRC testing scenarios, however, stop durations were not considered in the rules implemented in the test data.

(d) Check whether the valve/hatch is open

In conjunction with the stop location (scheduled or unscheduled), the most important signals for developing the detection rules were from the valve/hatch sensors. When neither a valve nor a hatch was opened, regardless of whether the vehicles were at a scheduled or unscheduled location, it would be virtually impossible for any fuel to be loaded or offloaded. Of course, this rule did result in the generation of a warning message when a valve or a hatch was opened at an 
unscheduled location. The level of warning, however, could not be determined with high certainty without further supporting information (evidence) from other detection rules. For instance, an initial warning (e.g., from a valve being opened at an unscheduled location) would be increased to a higher warning level if declining fuel levels were also detected.

(e) Determine whether there are any changes in marker concentration, fuel level, or gross vehicle weight

Sensors measuring fuel marker concentration and fuel level changes are important in determining changes in marker concentration and fuel quantity. Fuel integrity should always be consistent during the entire fuel delivery supply chain. That is, one can conclude that the fuel in question has been altered or diluted when a change of fuel marker concentration is detected. Changes in fuel quantity (i.e., fuel level), on the other hand, do not always indicate problems. Fuel level could be increasing or decreasing at a scheduled location, depending on whether the truck is loading or offloading at the time. Again, assuming that the dispatcher is always informed of location changes (even last-minute changes), fuel level changes during a scheduled stop are by themselves insufficient for detecting problems. Fuel level changes taking place at an unscheduled stop, however, should warrant a warning.

Besides sensors' readings of fuel marker and fuel level, vehicle weight could also be used as an added measure for monitoring fuel quantity changes. This information was collected along with the GPS and speed data by the DAS. Therefore, an additional detection rule could be added based on detected changes of the vehicle weights.

For the TRC field testing, a simple set of rules was designed based on measurements gathered from sensors for marker, fuel level, valve, and hatch. These rules combine the above actions a, $\mathrm{d}$ (for valve or hatch), and e (for marker and fuel level), as shown in the flowchart in Fig. 71.

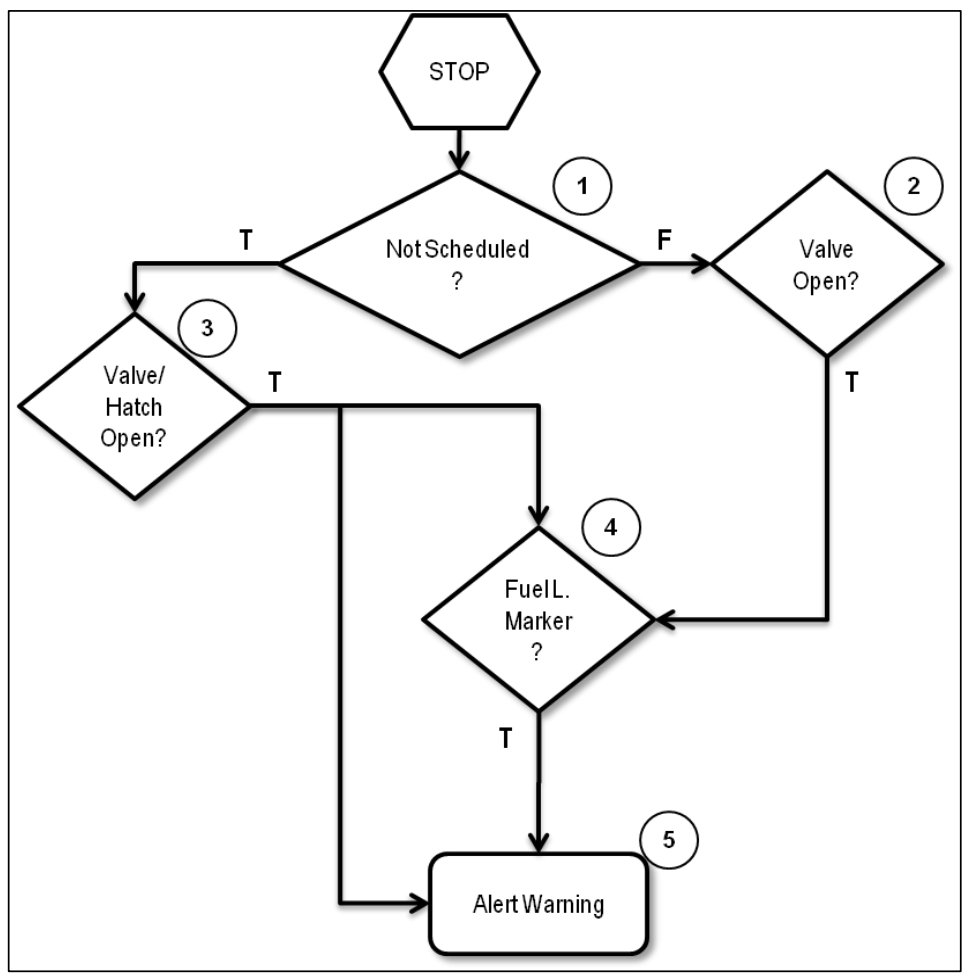

Fig. 71. Simplified decision rules for TRC testing. 
Specifically, the components of the flowchart have the following meanings:

- 1 -Is this an unscheduled stop?

- 2 - Is the valve opened given that the stop is right (i.e., scheduled stop)?

- 3 - Is the valve/hatch opened given that the stop is wrong (i.e., unscheduled stop)?

- 4-Is there any abnormal change in maker concentration or fuel level?

- 5-Warning level, result of the process.

Thus, the paths of combinations which should result in a warning (due to a deviation from a normal operation) are presented in Fig. 72.

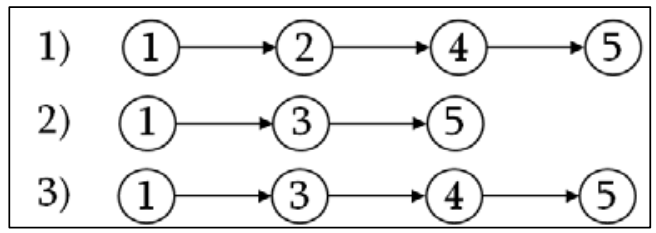

Fig. 72. Potential warning paths.

Path 1 indicates a scheduled stop in which a suspicious action happens only if there is a significant deviation from the normal operation in either marker concentration or fuel level. Path 2 indicates that if the driver makes a unscheduled stop and the valve/hatch is opened, then some level of warning should be immediately dispatched. Path 3 is the most critical one, which indicates that the driver not only opened the valve/hatch during a unscheduled stop, but there is also an unexpected variation in either marker concentration or fuel level.

Because of the design of the marker and fuel level sensors, which were installed around the fuel intake locations on the lower part of the tanker, there were no sensors associated with the hatch to measure fuel characteristics. Under Path 3, therefore, the determination of variations in marker concentration and/or fuel level measurements could only be conducted under the condition that the valve was open. In the event that a hatch was open (but valves were not) at an unscheduled stop, a warning should be sent immediately to the dispatcher. This is similar to Path 2.

The final output of the flowchart presented in Fig. 71 is a warning with certain warning level. Such degree of warning due to suspicious actions (in Paths 1 or 3 of Fig. 72) is based on two quantitative attributes (the marker concentration and the fuel level) and/or the three qualitative attributes (valve is open/closed, hatch is open/closed, and whether the stop is scheduled or not). In the determination of warning level, it was assumed that there is no uncertainty involved in the outputs of the valve and hatch sensors, whereas for the output of marker concentration and fuel level there might be uncertainties caused by noise variations in the measurements.

In the following subsections, the calculation of changes and the ranges of uncertainties in marker concentration and fuel level are described. A discussion of the method for combining the uncertainties of these variables and the valve/hatch sensor outputs to come up with a degree of suspicion follows.

\subsubsection{Determining Uncertainties in Marker Concentration and Fuel Level Measurements}

Since measurements of marker concentration and fuel level of the test vehicle could only be read when fuel was moving through the valve opening (i.e., when the valve was open), sensor measurement data to be used for the detection analysis is thus taken during this period. To avoid the high level of noise in the sensor signals caused by initial pressure generated when fuel flows through the valve opening, it was thought that data collected toward the end of the fueling/offloading might provide a less noisy signal for 
data analysis purposes. Thus, variations in marker concentration and fuel level data at each stop were calculated in two steps.

1. Calculate the median of a sample of data points within a small time period right before the valve is closed.

2. Calculate the relative change by comparing the variation observed by the current and previous measurements $\left(\mathrm{X}_{\mathrm{t}}\right.$ and $\left.\mathrm{X}_{\mathrm{t}-1}\right)$, against the reference value $\mathrm{X}_{0}$, according to the following Eqs. (1) and (2).

For an unscheduled stop, the observed variation was calculated as follows:

$$
\Delta \mathrm{X}=\left|\mathrm{X}_{\mathrm{t}}-\mathrm{X}_{\mathrm{t}-1}\right| / \mathrm{X}_{0}
$$

For a scheduled stop, the observed variation was calculated using the following equation:

$$
\Delta \mathrm{X}=\left|\mathrm{X}_{0}-\right| \mathrm{X}_{\mathrm{t}}-\mathrm{X}_{\mathrm{t}-1}|| / \mathrm{X}_{0}
$$

As a reference, Fig. 73 shows an example of a hypothetical profile of fuel level where the data points corresponding to the period in which the valve is open are highlighted and the portion of data used for calculation are indicated by the time widows denoted by $t w_{t}$.

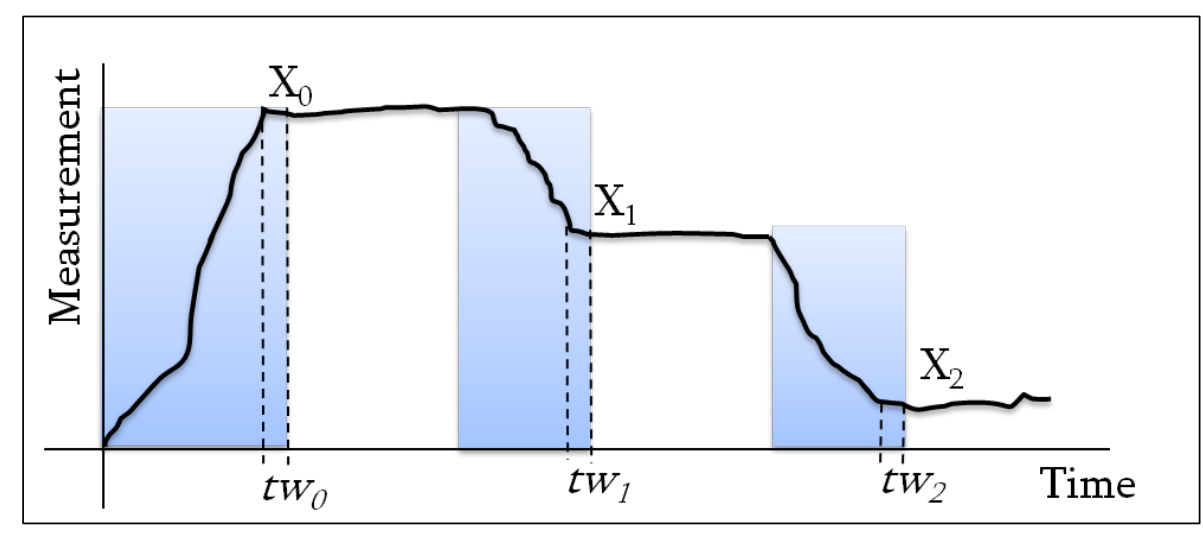

Fig. 73. Example of fuel level profile.

Under a normal operation, the relative change should lie within an expected error bound $(\varepsilon)$ of the sensors, $\Delta \mathrm{X}<\varepsilon$. Any deviation outside this bound points to a suspicious action. However, since the equipment noise is a random variable, a value dependent on real-world implementation such as mounting location as well as the sensor electronics, there is no certainty that such anomaly truly represents an illegal act. In this case, one should estimate the probability of an anomaly, $p\left(\Delta \mathrm{X}_{\mathrm{i}}>\varepsilon_{\mathrm{i}}\right)$, for each variable $i$ and combine all evidences (probabilities) to provide a degree of warning. In this context, the marker concentration and fuel level measurements are assumed to be two independent variables.

In an attempt to capture the uncertainty in the measurement of attribute $i$, the error bound is allowed to vary between two critical values: the most likely error denoted by $\varepsilon_{\operatorname{min.i}}$ and the worst deviation error denoted by $\varepsilon_{\text {max,i }}$. The bound $\varepsilon_{\text {min,i }}$ is the minimal expected error of attribute $i$, representing a situation of highest uncertainty with respect to an illegal operation, whereas $\varepsilon_{\max , \mathrm{i}}$ should represent a situation in which an illegal act is very likely. All information provided by the marker concentration sensors, fuel level sensors, and qualitative attributes was aggregated through an evidential reasoning algorithm as briefly described in the next section. 


\subsubsection{Basic Algorithms}

To combine the qualitative and quantitative information in the evidential reasoning approach, a commonscale grade was needed to classify the input measurements. To this end we used a utility function, in which the various inputs were given different weights to determine an overall warning level.

For a given quantitative attribute, the different levels of uncertainty were defined by dividing the error bound $\left[\varepsilon_{\min , \mathrm{i}}, \varepsilon_{\max , \mathrm{i}}\right]$, as defined in Sect. 5.5.3, into five quantitative grades, with each category $n$ represented by a bin number $F_{n}$ and an associated utility value $u\left(F_{n}\right)$ (utility of grade $F_{n}$ ) between 0 and 1. The utility is a way to grade the different levels of error in terms of likelihood. If $\varepsilon_{\mathrm{i}}$ is very large, $p\left(\Delta \mathrm{X}_{\mathrm{i}}>\varepsilon_{\mathrm{i}}\right)$ should be very small, and therefore the likelihood of a deviation from normal operation is very likely. The main idea is that if a deviation occurrence is improbable and it still happens, it should be highly investigated.

The relationship between the utility and the grade can be found from empirical data or assigned subjectively based on experience. Since no empirical data was available at the time this relationship was established (for testing purposes), it was assumed to be a linear relationship, as shown in Fig. 74. Notice that $F_{1}=\varepsilon_{\min , \mathrm{i}}$ and $F_{2}=\varepsilon_{\max , \mathrm{i}}$.

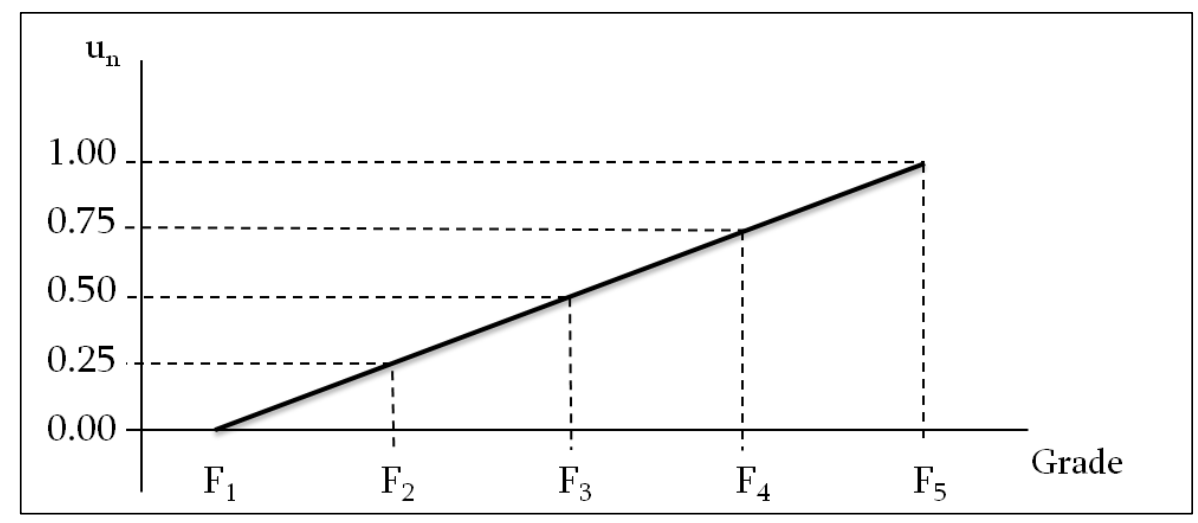

Fig. 74. Linear utility function.

For the FTE evidential reasoning algorithm, the utility is interpreted as the likelihood of a questionable event. Therefore, higher utilities are associated with higher likelihoods of illegal acts. In this case there are five classes (grades) of likelihood outcomes: very unlikely, unlikely, moderate, likely, very likely.

With respect to the qualitative attributes, it was assumed a simple scale in which the worst case was assumed for circumstances such as either the valve/hatch being open or the stop being unscheduled, resulting in $u_{5}=1$ (or very likely). Otherwise, the best case was assumed, resulting in $u_{1}=0$ (very unlikely). There were no intermediate categories for these attributes.

Uncertainties about an input value were represented by degrees of belief. Let $\beta_{n, i}$ be a degree of belief associated with the utility $u_{n}$ for attribute $i$. Using the defined categories, any input value is then translated into a distribution of beliefs defined as follows.

Let $e=\left\{e_{1}, \ldots, e_{i}, \ldots, e_{L}\right\}$ be the set of input values for each attribute. An assessment for an attribute $i$ is represented by the following utility distribution:

$$
\mathrm{S}\left(e_{i}\right)=\left\{\left(u_{n}, \beta_{n, i}\right), n=1, \ldots, N\right\}, i=1, \ldots, L,
$$


where $L$ is the number of attributes (which is equal to 5 in the case of this project) and $N$ is the number of categories (also equal to 5).

The evidential reasoning algorithm combines the five distributions, defined by $\mathrm{S}\left(e_{i}\right)$, to generate the aggregated beliefs denoted by $\beta_{n}$. The final result of the evidential reasoning algorithm is an average utility value. Since this problem is “complete” with regard to evidential reasoning algorithm, $\sum \mathrm{n} \beta_{n}=1$ (all beliefs add up to 1), the average utility or utility of the set of the input values given by the set $e$ is given by

$$
u(e)=\sum n u(F n) \cdot \beta n
$$

\subsection{INTEGRATING THE GEO-FENCING AND EVIDENTIAL REASONING RULES}

\subsubsection{Initial Setup with Passenger-Car Simulation Runs}

Initial testing of GPS data collection using the DAS began in late October 2010. The process started with the selection of several Pilot stations in the area, ranging in distance from 5 miles to 30 miles away from where the ORNL team was located. A route would begin at a simulated terminal or a Pilot station centrally located among the Knoxville terminals, and it would end at a Pilot station in the greater Knoxville area. Data was logged from terminal to station and upon arrival at a destination; the fuel and marker levels were manipulated (using the test box described in Sect. 4 to simulate the delivery of a load of fuel). Figure 75 shows a route (based on GPS data collected with a passenger car) from the terminal marked on the right side to a Pilot station approximately 5.5 miles from it.

As an added check to evaluate the accuracy of the GPS data collected by the DAS, a Trimble Juno SB GPS unit was also used during the initial simulation runs. Due to tree cover, signal strength, multipath errors, and similar issues that arise when working with GPS devices, measurements for a small handful of areas were slightly off between the two instruments. However, the overall geographic display provided by the two systems closely overlaid each other. Figure 76 illustrates the two networks.

\subsubsection{Additional Passenger Car Simulation Scenarios on Actual Truck Routes}

In March 2011, the DAS system was used to collect additional data for more structured simulation runs. This time, seven different scenarios were constructed to depict more realistic situations which tanker truck drivers could encounter during their delivery operations. In addition to a control scenario where the

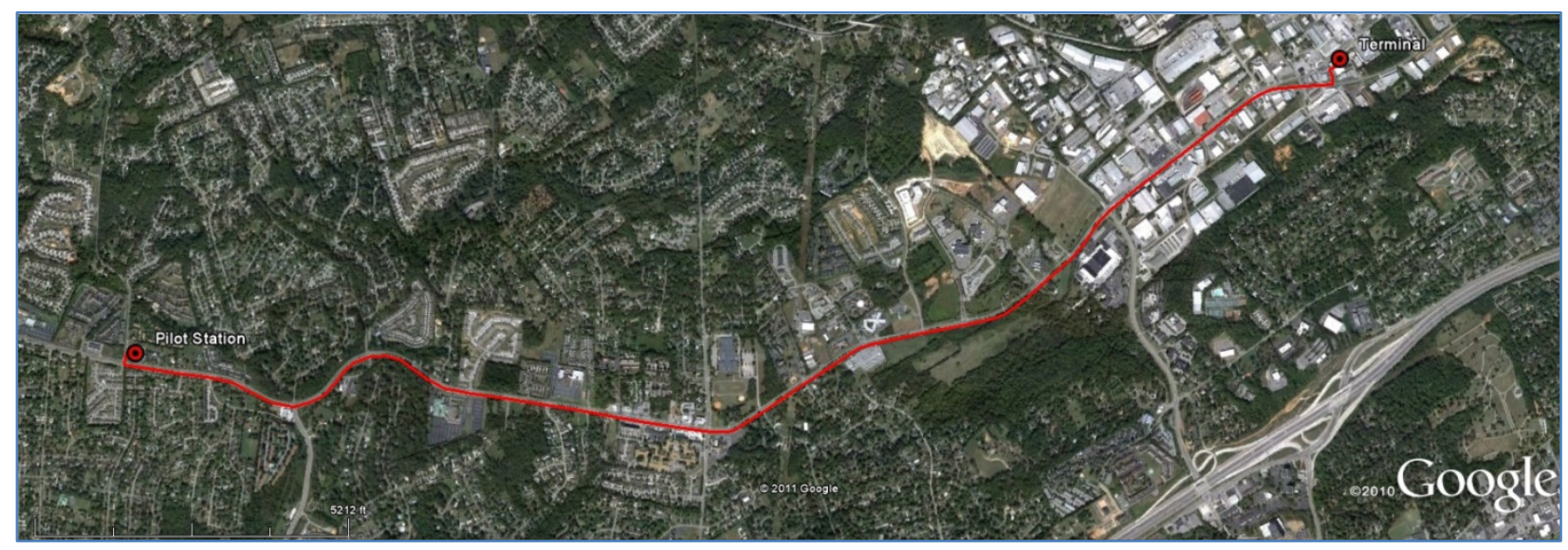

Fig. 75. Example route for a simulation run in Knoxville, Tennessee. 


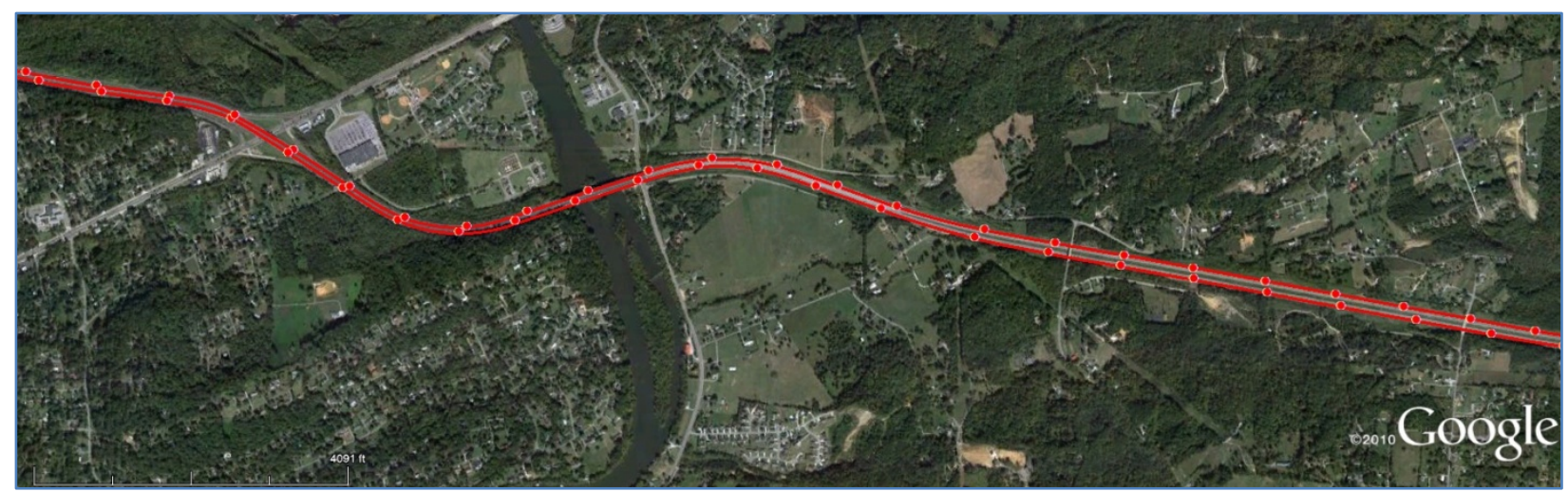

Fig. 76. A comparison of spatial location accuracy in data collected with the DAS device (points) and a commercial Trimble unit (lines). Two travel directions are displayed.

delivery took place as scheduled by the dispatcher, scenarios that simulated fuel theft, jurisdiction crossing, blending, unplanned detours, and a vehicle breakdown were simulated. Actual truck route data was extracted from the Pilot PeopleNet Fleet Manager system and integrated to form the realistic driving behaviors for these simulations. Since no actual sensor was available for the passenger car simulation, the Sensor Simulation Unit was used to simulate sensor readings (marker concentration, fuel level, and valve and hatch position) in these scenarios.

An example of one of these mock deliveries is displayed in Fig. 77. In this instance, a driver was assigned to make a delivery to the station on the east side of the map. His assigned route is outlined in green. However, the driver deviated from this path, followed the red route instead, and made a stop in the irregularly shaped area shown in the northeast corner. The location of this shape, in fact, is a mall parking

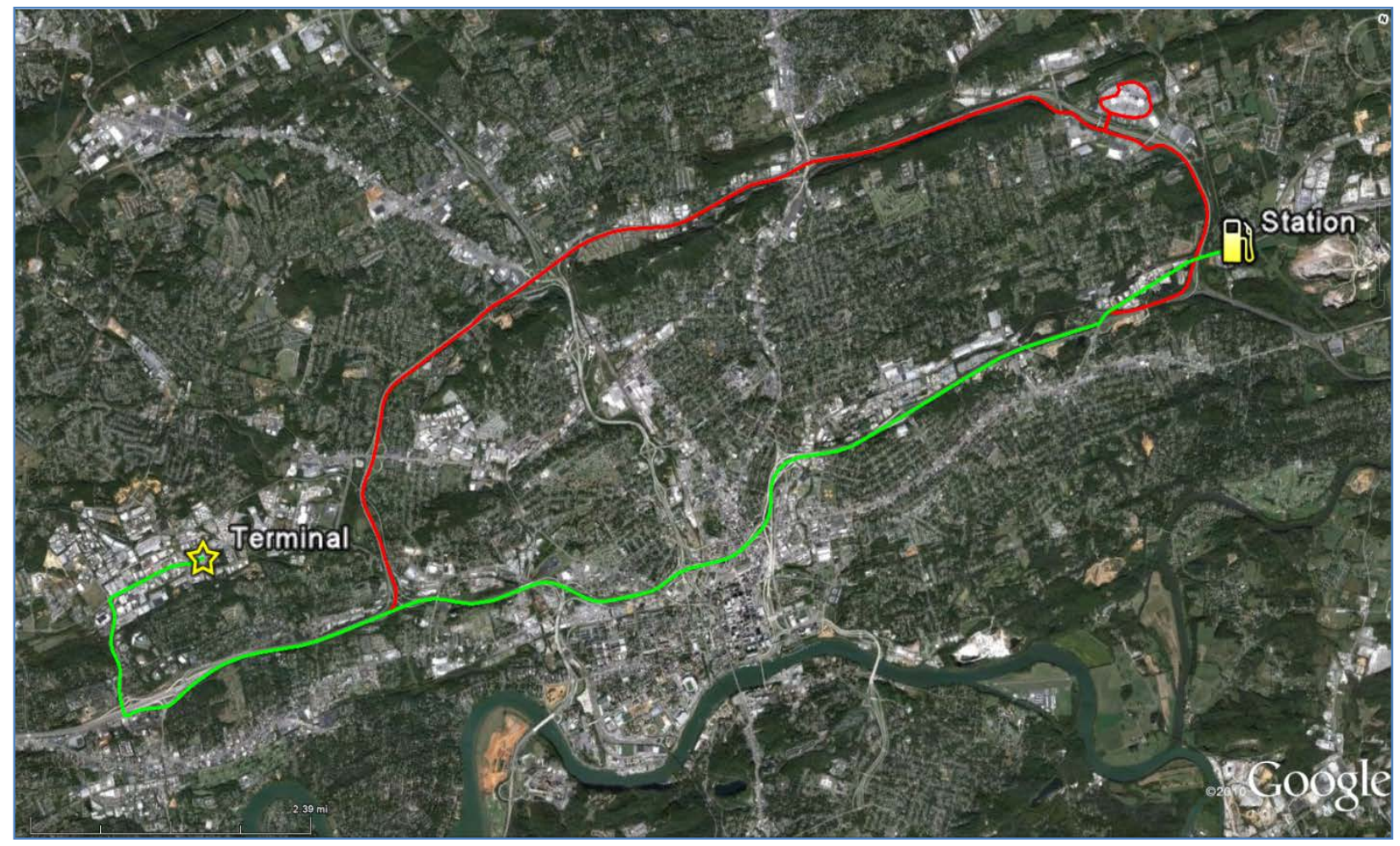

Fig. 77. Example of mock delivery. 
lot. To make things even more suspicious, the fuel levels in one of the tanker compartments increased, while the marker levels decreased, indicating that the driver was blending unauthorized substances into his fuel load.

Because realistic sensor data was not available for these simulations, these cases were used only to examine driving behaviors and explore how geo-fencing could be applied to identify abnormal patterns. In the example shown in Fig. 77, the red route could have reasonably been a legitimate alternative route to the original green route if the truck had not stopped at the parking lot and opened the valve (simulated) that resulted in a change of fuel integrity (simulated). This illustrated one of the complexities encountered when designing a geo-fencing algorithm: the availability of multiple routes between a pair of origin and destination points. This reemphasizes that geo-fencing alone would likely provide insufficient evidence to detect FTE. 


\section{TEST TRACK ACTIVITIES}

The POC testing did not lend itself to real-world data collection due to the non-production (not certified for hazmat tanker use and not environmentally hardened) nature of the equipment and sensors used, and the need to control the testing steps and maneuvers to gather the required data. These factors and the general concern for staff safety and the safety of the Pilot vehicle led to a decision early in the project to conduct this field vehicle-based data collection at a closed test facility. The TRC in East Liberty, Ohio, was chosen based on the size of their facility (several miles of roadway), their experience with commercial vehicle testing, and their ability to handle the required fuel transfers.

\subsection{PRELIMINARY TESTING}

Prior to the field test with the tanker, preliminary testing was conducted at TRC to test out the marker sensor hardware and software under conditions similar to those which would be experienced during the field test; this opportunity was also taken to test the prototype T2TCU with a functioning marker sensor. Additionally, the simulated sensor data was collected as the test scenarios were performed on-site at TRC to confirm the DAS-to-BOS communications and collect data to fine-tune the evidential reasoning system.

\subsubsection{Fuel Loop Sensor Testing}

The fuel loop test rig was constructed to allow fuel from a vehicle-mounted fuel tank to be pumped out of the tank's output connection, through a $6 \mathrm{ft}$ long, 4 in. diameter aluminum pipe, and back into the tank's input connection. The fuel was circulated at $60 \mathrm{gal} / \mathrm{min}$ (gpm). The section of aluminum pipe was chosen because it matched the diameter and material of the input and output fuel lines on the tanker vehicle identified for the field test.

Prior to testing, major preparations were successfully carried out for the doping of the fuel marker into diesel at loop testing field trials. For transport of concentrated marker solutions, 10 vials, each containing $40 \mathrm{~mL}$ of highly concentrated marker solution, were used to sequentially dose in marker dye at the test track fuel loop setup. Each vial contributed slightly less than $100 \mathrm{ppb}$ (mass: mass basis) of fuel marker dye, with the total exact amount added reaching about $1000 \mathrm{ppb}$, or $100 \mathrm{ppm}$. The total amount of marker used, in terms of mass, to dope 150 gal of fuel, was less than 500 milligrams, which was measured out to go into the concentrated doping solutions on an analytical microbalance accurate to milligram quantities.

The fuel loop testing conducted at TRC enabled the first field evaluation of both the ORNL-designed sight glass and the marker sensor. The 4 inch diameter aluminum pipe was prepared with a flanged central section. This flanged section made it possible to attach a custom sight glass in-line. This sight glass permitted the fuel marker sensor to be mounted and to monitor the fuel marker concentration levels within the pipe. To facilitate the testing, TRC constructed a rig which enabled fuel to flow through the instrumented sight glass in a continuous loop. This apparatus is shown in the test setup in Fig. 78, with a close-up of the glass and marker sensor. The high optical quality of the acrylic is evident in this figure.

The fuel circulated through the fuel loop was initially unmarked (marker concentration levels of $0 \mathrm{ppb}$ ). After an initial baseline measurement, a small quantity of marker was introduced into the fuel. The marker was contained in a $40 \mathrm{ml}$ vial that contained highly concentrated marker dissolved in a small amount of diesel. The contents of the vial were added to the circulating fuel via a small vacuum inlet line. Once the contents were thoroughly mixed and diluted within the circulating fuel (of which there was 350 gal), a small concentration of fuel marker resulted. Enough vials of marker were prepared to produce 10 increasing levels of marker concentration in steps of approximately $100 \mathrm{ppb}$. Tests were conducted with a 60 gpm flow rate. 


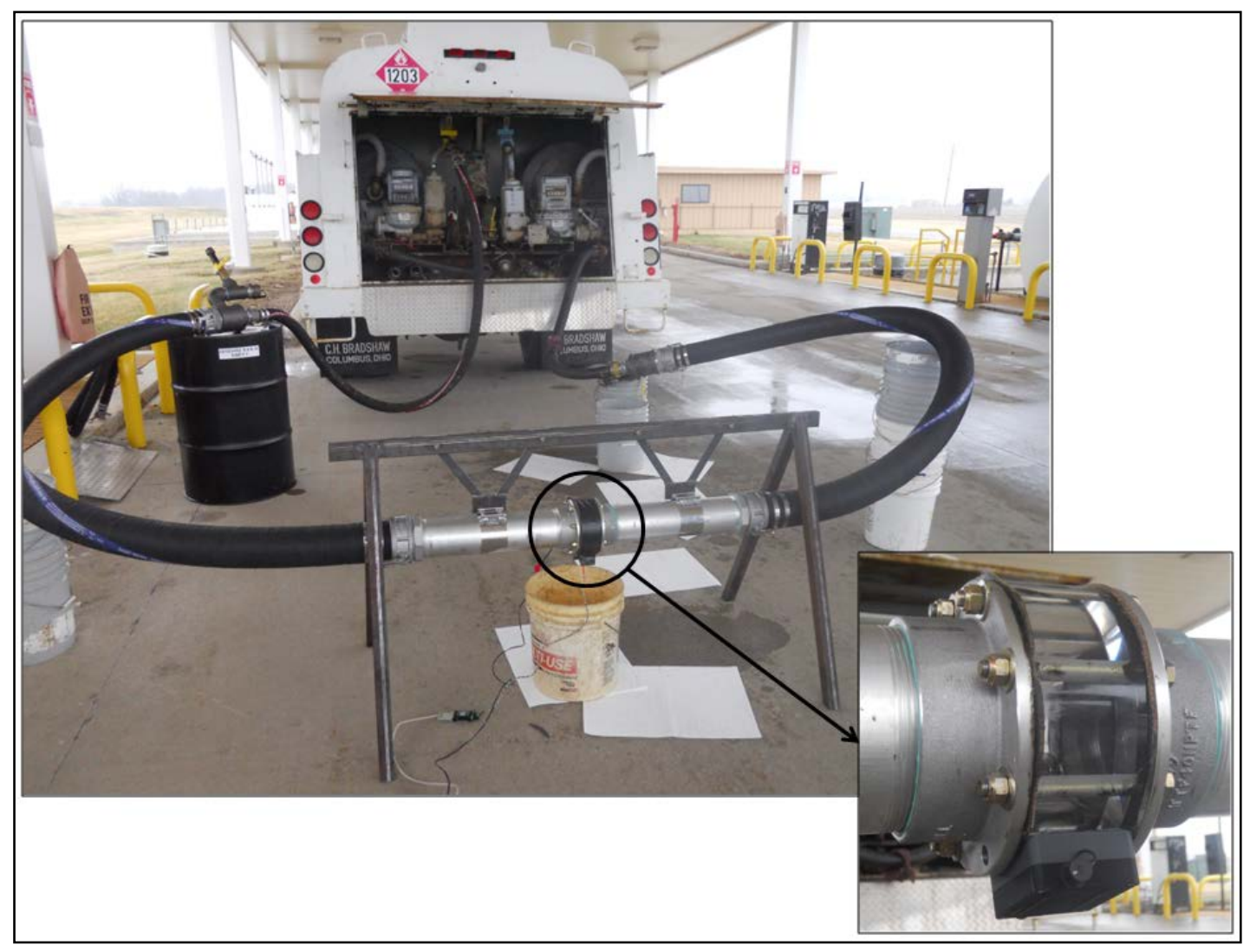

Fig. 78. Fuel loop test rig.

As the marker vials were added, the in-line prototype fuel marker sensor recorded all changes in the marker concentration by monitoring the recirculating fuel. For each added vial, a short period of the fuel flowing through the loop was recorded. This was followed by a short period of the fuel remaining static within the rig (i.e., non-flowing). The data collected during this experiment is shown in Fig. 79. The testing conducted with the fuel loop revealed that steady flow of the fuel had little impact on the sensor reading, and that no leak problems were caused by the modified Truck Trailer Manufacturer's Association bolt pattern on the ORNL sight glass. The raw data shown in Fig. 79 indicates that under field conditions the current generation marker sensor was able to measure the concentration of the fuel marker with a resolution of at least $\pm 100 \mathrm{ppb}$ (12.5\% of the anticipated $800 \mathrm{ppb}$ marker level in the field tests).

In this figure, (a) shows the sensor output as the marker concentration was increased from 0 to 1,000 ppb in $100 \mathrm{ppb}$ increments and (b) is a magnified view of a portion of the data in (a), showing how the signal settled to a steady value after mixing of the marker. Between the first and second portions of the test (identified in the preceding figure as “Testing Paused"), the marker concentration read by the sensor increased. This was thought to be caused by two factors. First, the test rig apparatus (particularly transitions between different cross sections of flow) lead to some of the highly concentrated marked fuel getting trapped in dead spots prior to fully mixing. This caused the apparent increase in concentration after the break in testing (after which the concentration stabilized before the next vial of marker was added): the marker was better dispersed during the break. Second, when the highly concentrated fuel was first introduced into the tank, it likely splashed onto the tank walls. Over time, these droplets slowly drained/dripped back into the fuel mixture. These situations were corrected for the mixes performed in preparation for the field testing. 


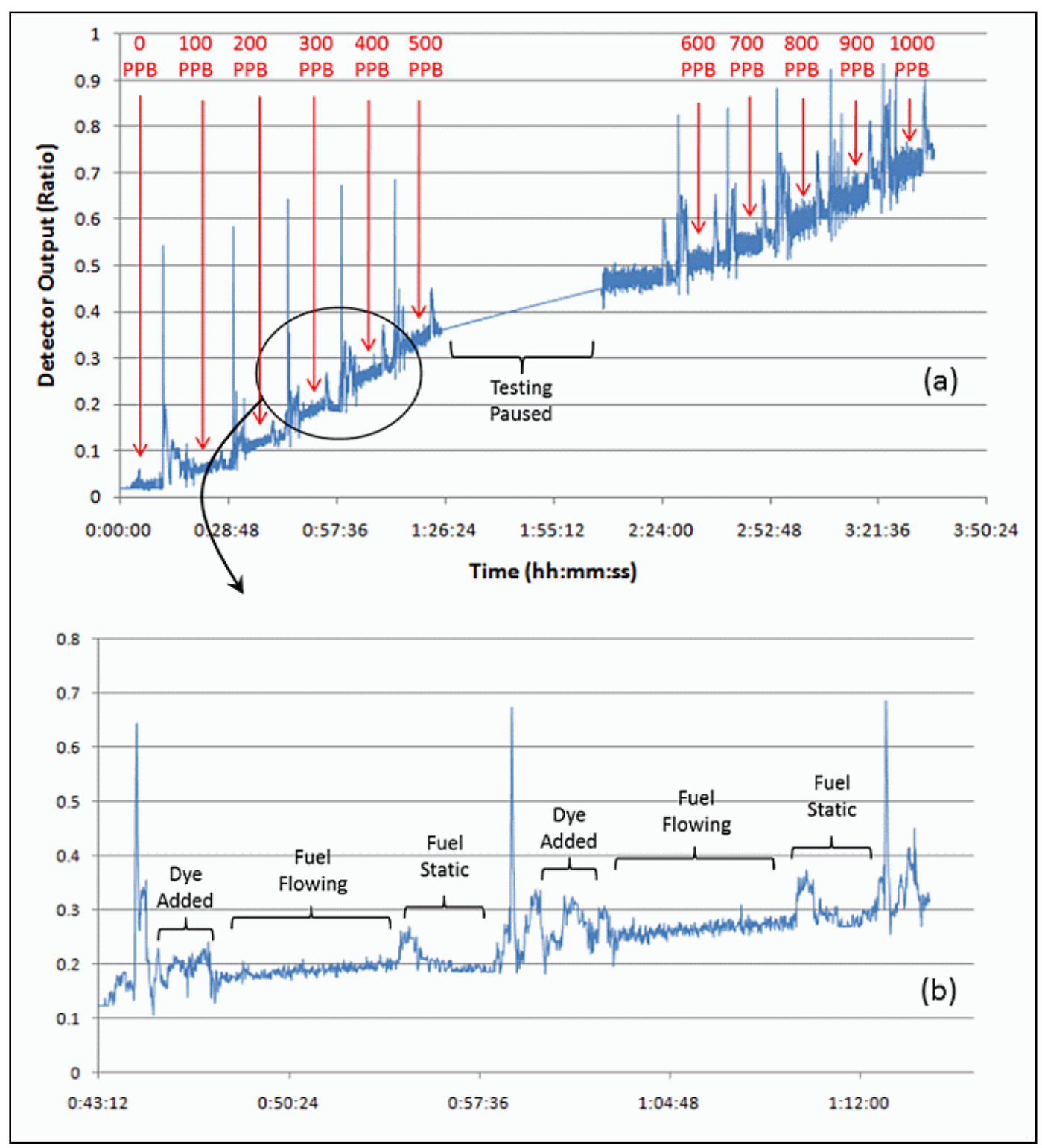

Fig. 79. Marker sensor data from fuel loop test at TRC.

The data collected during this test provided three useful pieces of information. First, the data showed that the sensor worked well in the environment anticipated for the upcoming field test. Plus, the resolution of the sensor in this environment could easily detect changes on the order of $100 \mathrm{ppb}$ on either flowing or static fuel (Fig. 80).

Secondly, the data showed that the mixing of highly concentrated fuel into a larger reservoir of unmarked fuel required significant recirculation before the marker became fully blended. The increasing marker concentration experience with additional mixing can be seen in Fig. 79-where the marker concentration is seen slowly increasing from one vial to the next as the fuel is recirculated.

Thirdly, the fuel loop test demonstrated that the gain of the detector's amplifiers could be made significantly higher (times 6) to reduce the sensor's signal-to-noise ratio. This technical detail was important for optimizing the sensitivity and range of the sensor. 


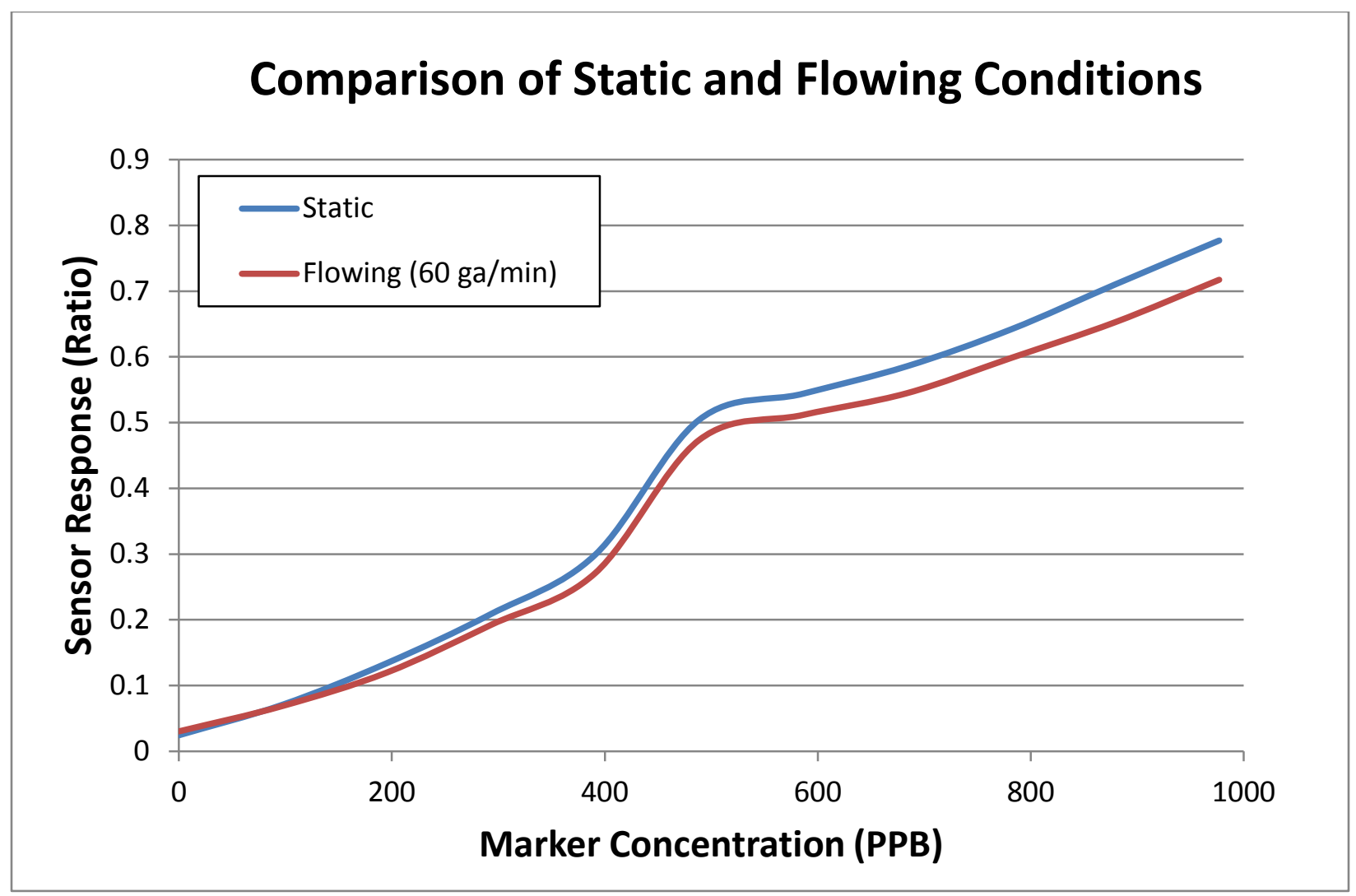

Fig. 80. Marker concentration data.

Following the completion of this experiment, some minor changes were made to the circuitry of the prototype fuel marker sensor to increase the gain of the sensor.

Additionally, the DAS and T2TCU used in the tanker testing were connected to the optical sensor, and data was collected at flow rates of 60 and 120 gpm for a marker concentration of 1,000 ppb (Fig. 81). The data collected using this methodology confirmed the reliability of the T2TCU in correctly reporting the sensor readings to the DAS.

\subsubsection{Passenger Car Communications Testing}

In order to test the communications link at the test track, the test scenarios developed for the pilot test were implemented using a test box in to simulate sensor readings (Fig. 82). This test setup made use of the analog and digital data collection capabilities of the DAS (in conjunction with GPS) to generate streaming data identical to that of the real test.

During this testing, scenarios involving the four locations selected by TRC (simulated fuel rack and delivery points) were implemented with this equipment installed in a passenger car. The collected data was sent back to ORNL wirelessly and manipulated by the data retrieval tool and submitted to the evidential reasoning system's server in 5 min increments. The testing successfully demonstrated the realtime communication between the DAS and ORNL's server using the FTE Upload Data Service software developed for that purpose. 


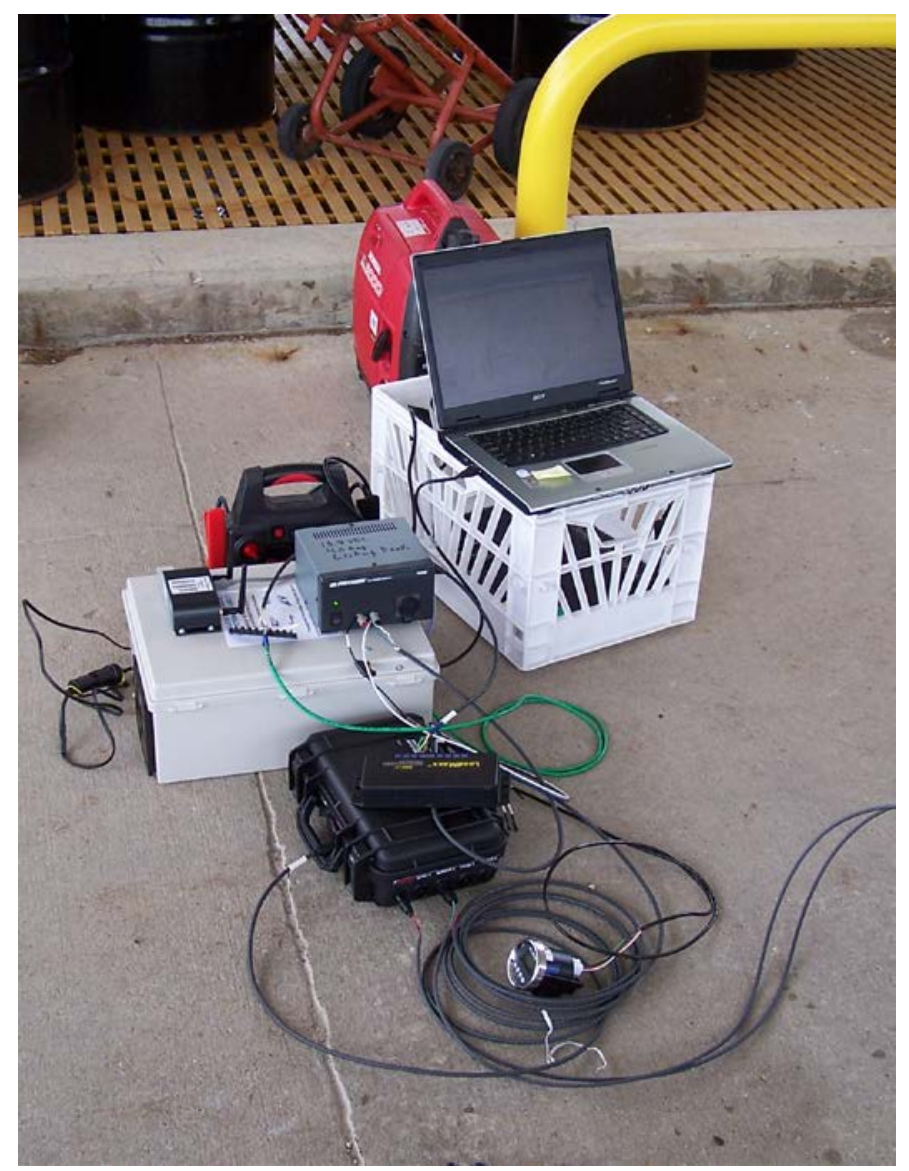

Fig. 81. DAS and T2TCU testing with fuel loop.

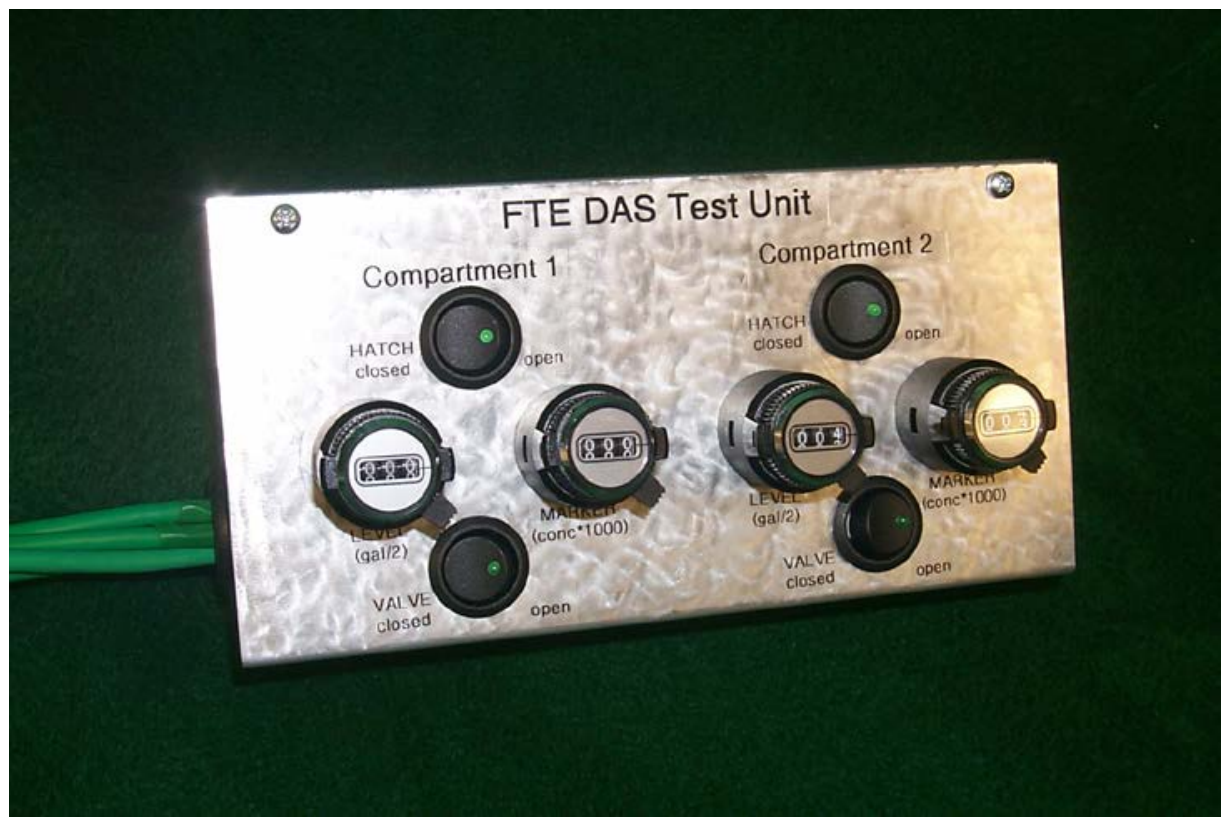

Fig. 82. FTE sensor simulation unit. 
Data collected from these test scenarios was analyzed upon returning to ORNL. Figure 83 shows the routes (using data on GPS locations) inside the TRC that were used during the pre-testing. An example of the measure from sensors (simulated) is given in Fig. 84. As planned, the GPS and sensor response data were collected at 10 second intervals and transmitted as a packet at the rate of 5 min intervals. During the post-data examination, the 5-min data retrieval intervals proved sufficient, but it was discovered that some sensors' responses could be "missed" due to short duration of the simulated activities. Therefore, the sample rate was increased to $1 \mathrm{~Hz}$ for the tanker testing.

\subsubsection{Pretest results from the passenger car testing}

Since the pretest was mainly for the purpose of conducting the fuel loop testing, no tanker truck was actually presented. A passenger car with the FTE sensor simulation unit, a test box with manual dials to simulate sensor activities, was used to collect GPS and simulated sensor "responses" data. The DAS was powered by plugging in the passenger car, which caused certain unstable situations to the e-DAQ power connections. Nevertheless, data was collected as planned at every 10 second interval and packets of data were transmitted via cell communication back to the server (at ORNL) at a 5 min interval.

Due to issues from the power source inside the passenger car and the GPS blockages in certain testing areas within the TRC site, signals transmitted from the e-DAQ, through the communication link, were not as good as expected. These issues resulted in several data gaps in datasets stored on the server. Note that the communication issue does not generally affect the data stored on the DAS system itself.

\subsubsection{Review of GPS and simulation scenario data}

Using on-board stored data from the e-DAQ system, information collected from the pretest scenarios was post-processed after the team return to ORNL. Analysis was conducted using these post-processed data sets (i.e., data gaps filled). Based on GPS location data collected from the pretest, Fig. 85 shows the travel routes inside the TRC that were utilized during the pre-testing. An example of the measures from sensors (simulated) is given in Fig. 86. Obviously, loading and offloading activities can only occur within the time the valve is opened.

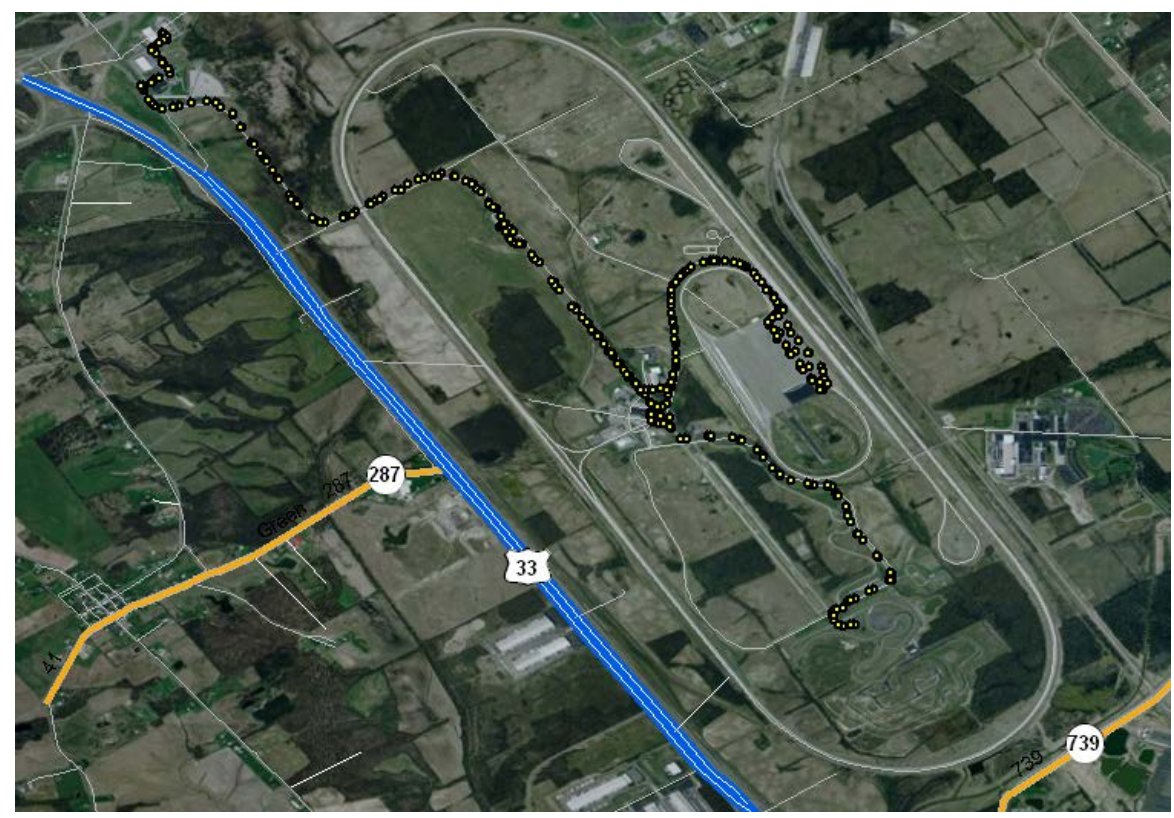

Fig. 83. Travel routes based on GPS data from communications testing. 


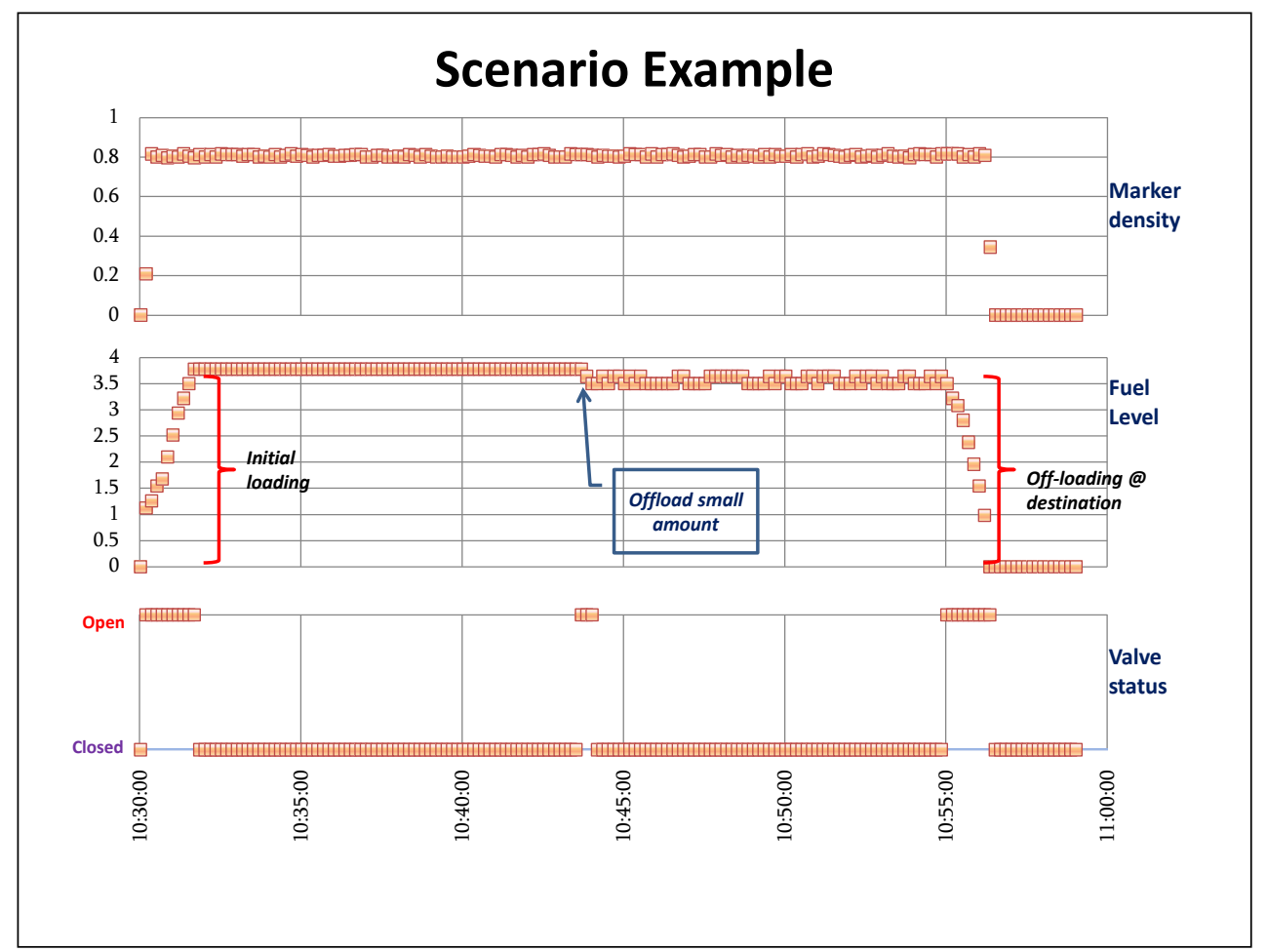

Fig. 84. Simulated data collected during communications testing at TRC.

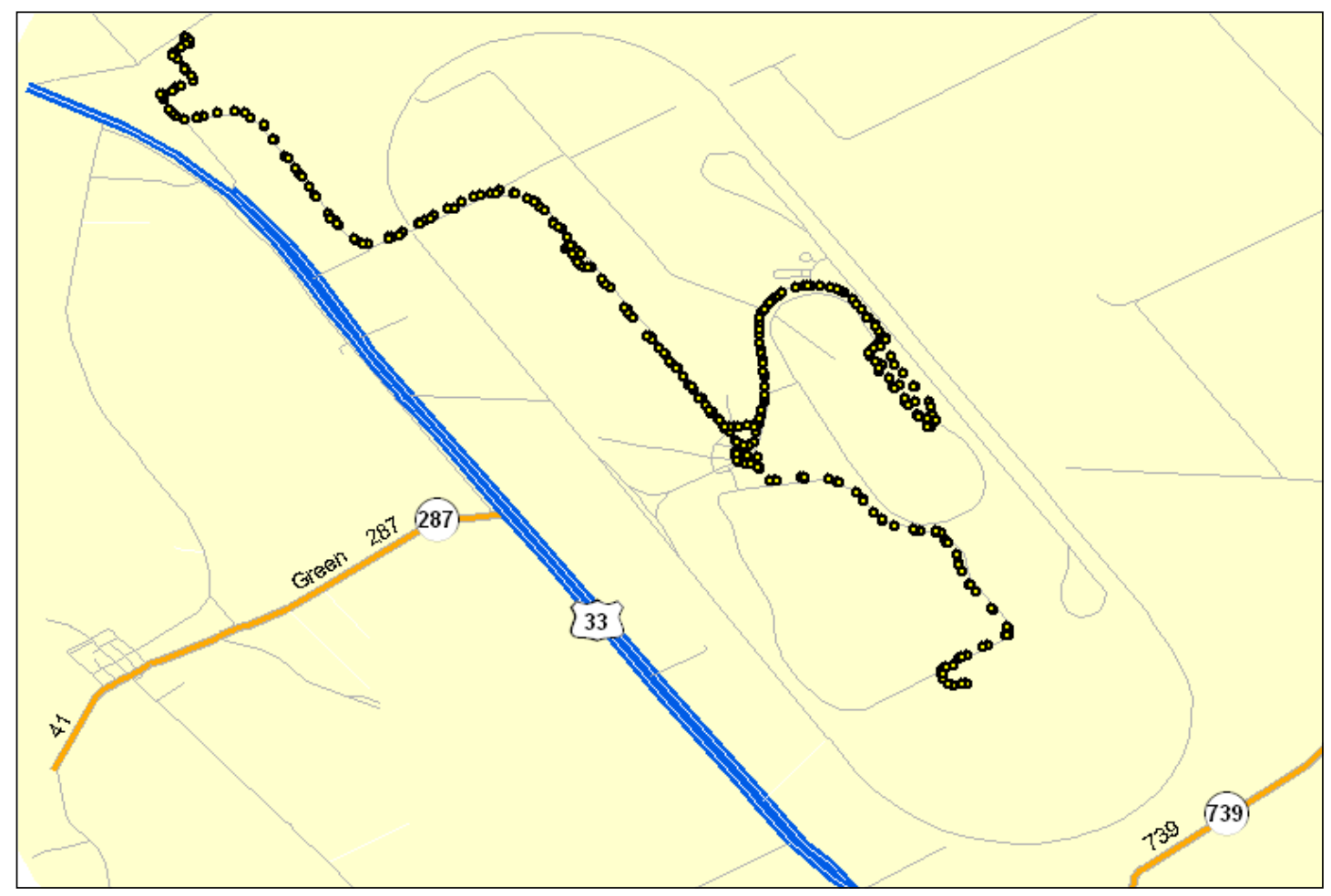

Fig. 85. GPS plots of the pre-test routes at the TRC on March 22, 2011. 


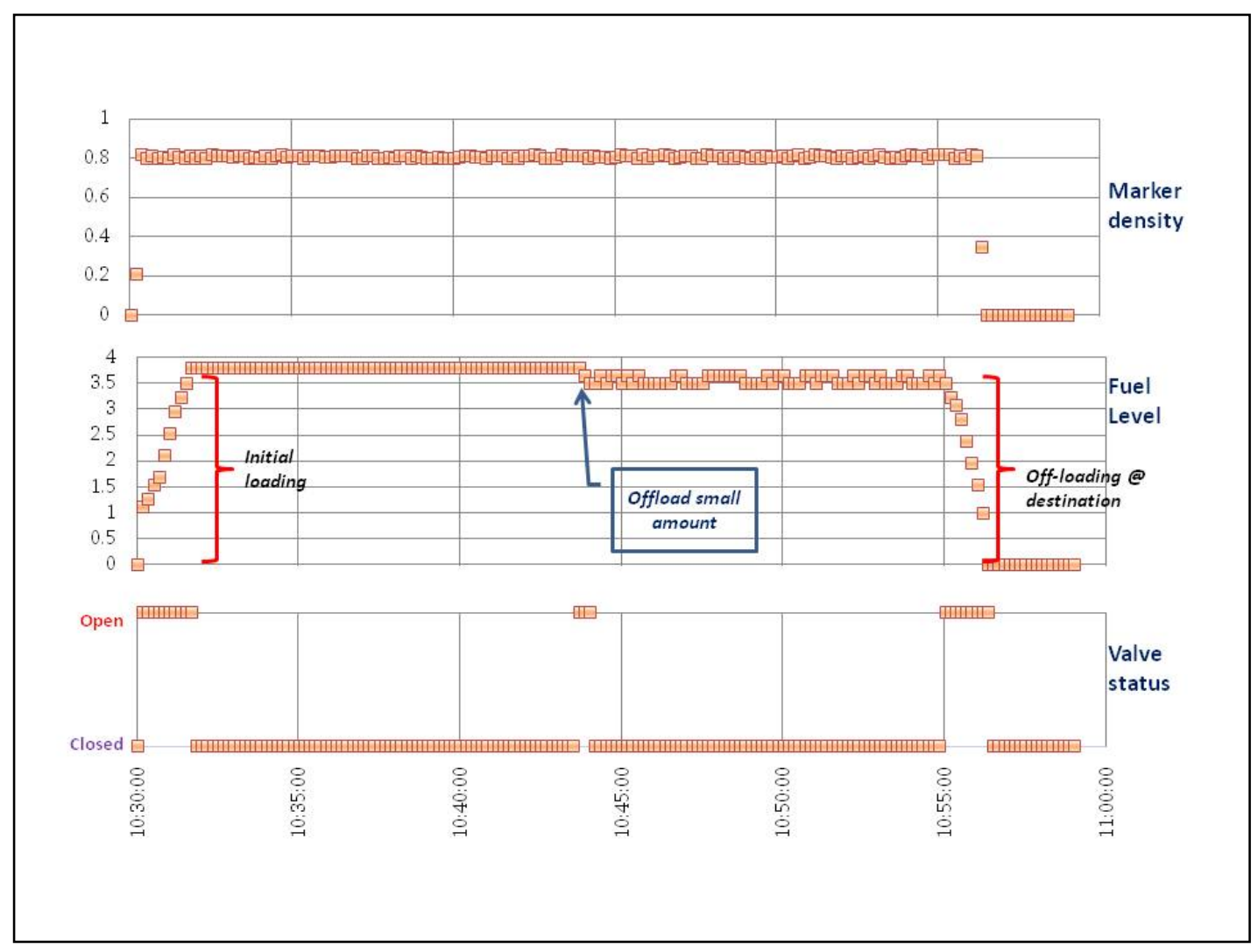

Fig. 86. Sample scenario results from the pre-test at TRC on March 22, 2011.

\subsection{FIELD TEST}

The Field Test conducted at TRC using the Pilot Travel Center's tanker tractor-trailer involved the full ORNL end-to-end FTE system. The test vehicle was moved from location to location within the TRC reservation to simulate loading and offloading locations such as a fuel terminal and retail fuel sales location and included several test scenarios. This testing took place in mid-April 2011 and required a period of system integration prior to the official testing, followed by a restoration of the test vehicle to its original condition.

\subsubsection{Integration}

ORNL staff arrived at TRC several days before the actual testing to oversee the upfitting of the Pilot tanker tractor-trailer (vehicle) by TRC staff. A technician from Air-Weigh also assisted. All tanker instrumentation was connected to the T2TCU, mounted above the valves on the trailer as shown in Fig. 87. A power connection was made to this unit, from which power would be distributed to other trailer instrumentation.

Each of the two instrumented compartments (front- and rear-most) had the sight glasses replaced with instrumented units to which were attached marker and pressure sensors. The sensors were tested to verify they were functioning properly (Fig. 88).

Switches were installed on the hatches and valves of these two compartments as well, as shown in Fig. 89 and Fig. 90. 


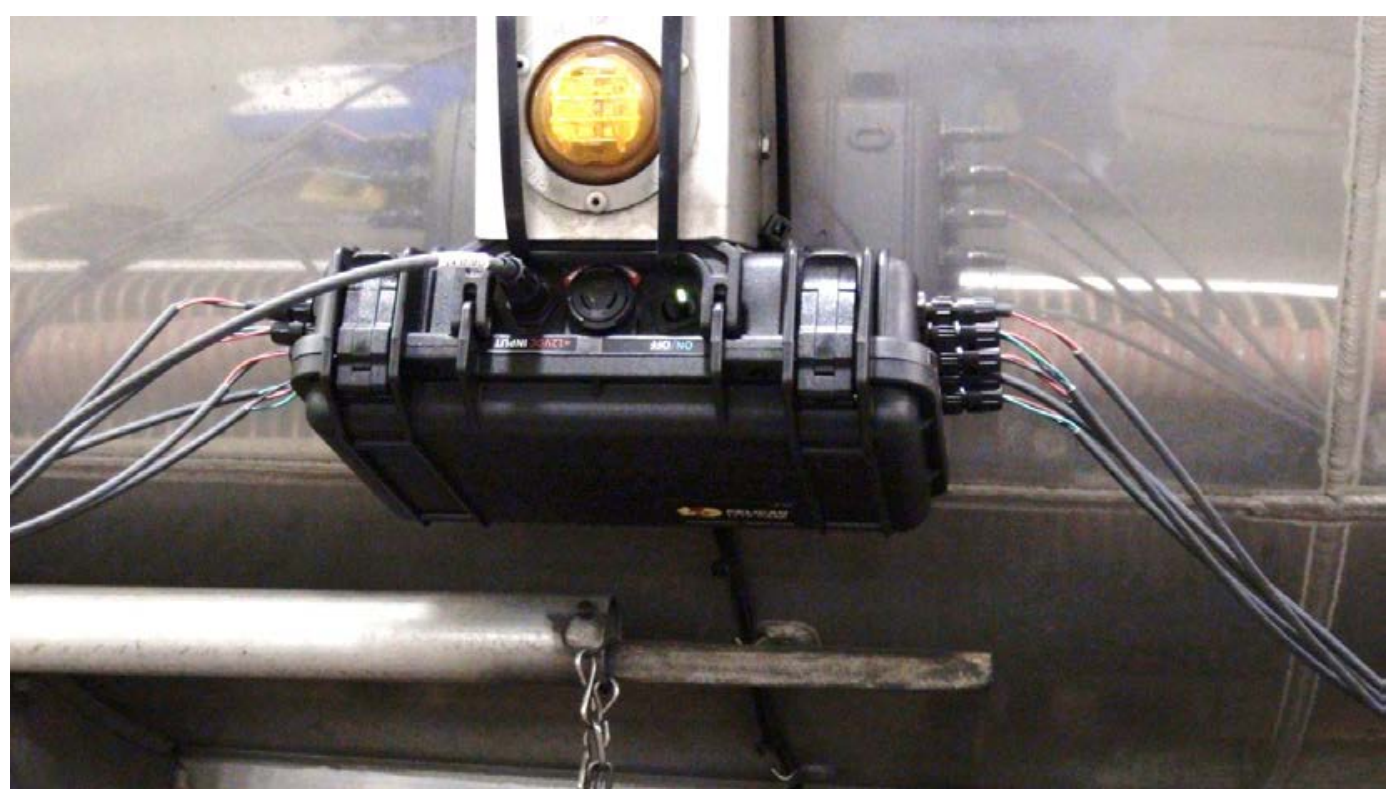

Fig. 87. T2TCU installed on tanker.

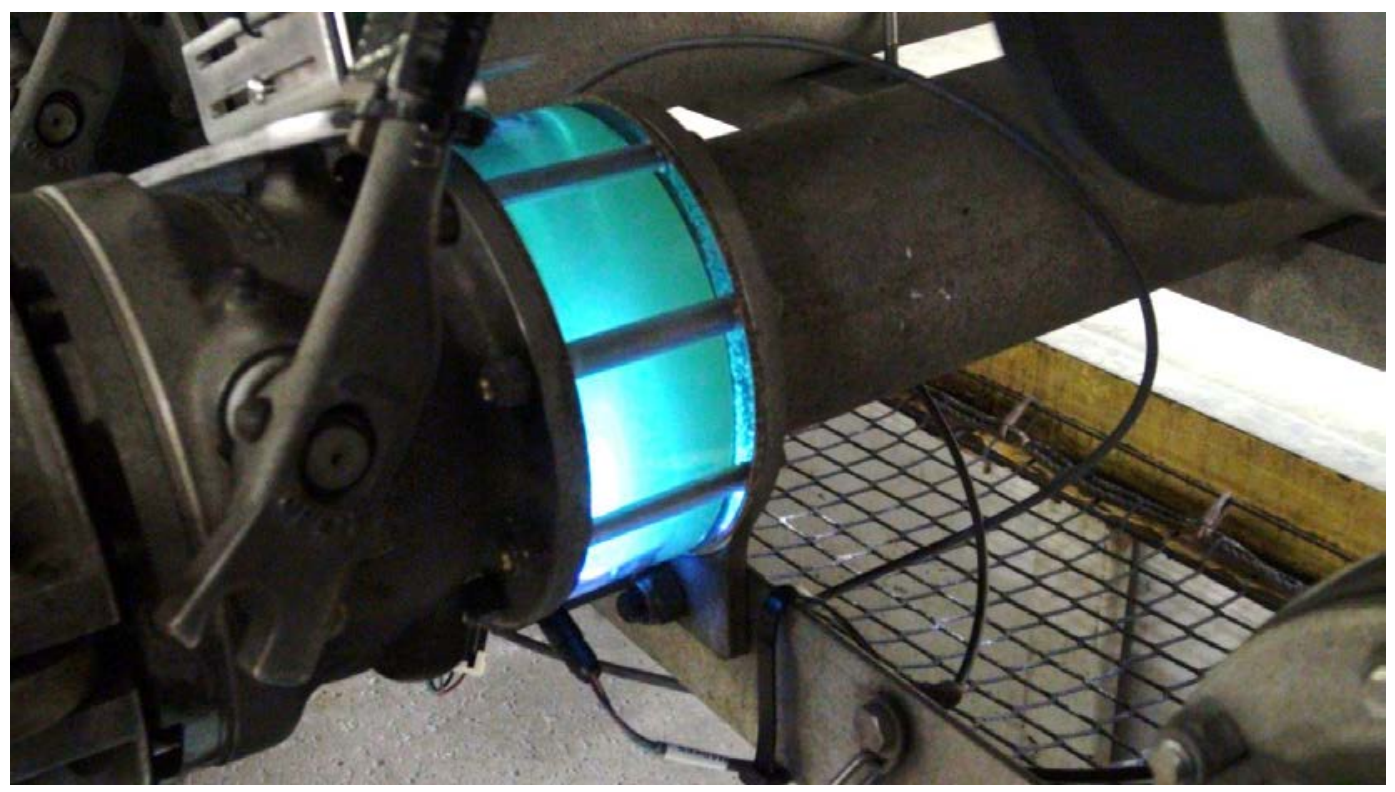

Fig. 88. Marker sensor in situ operation verification. 


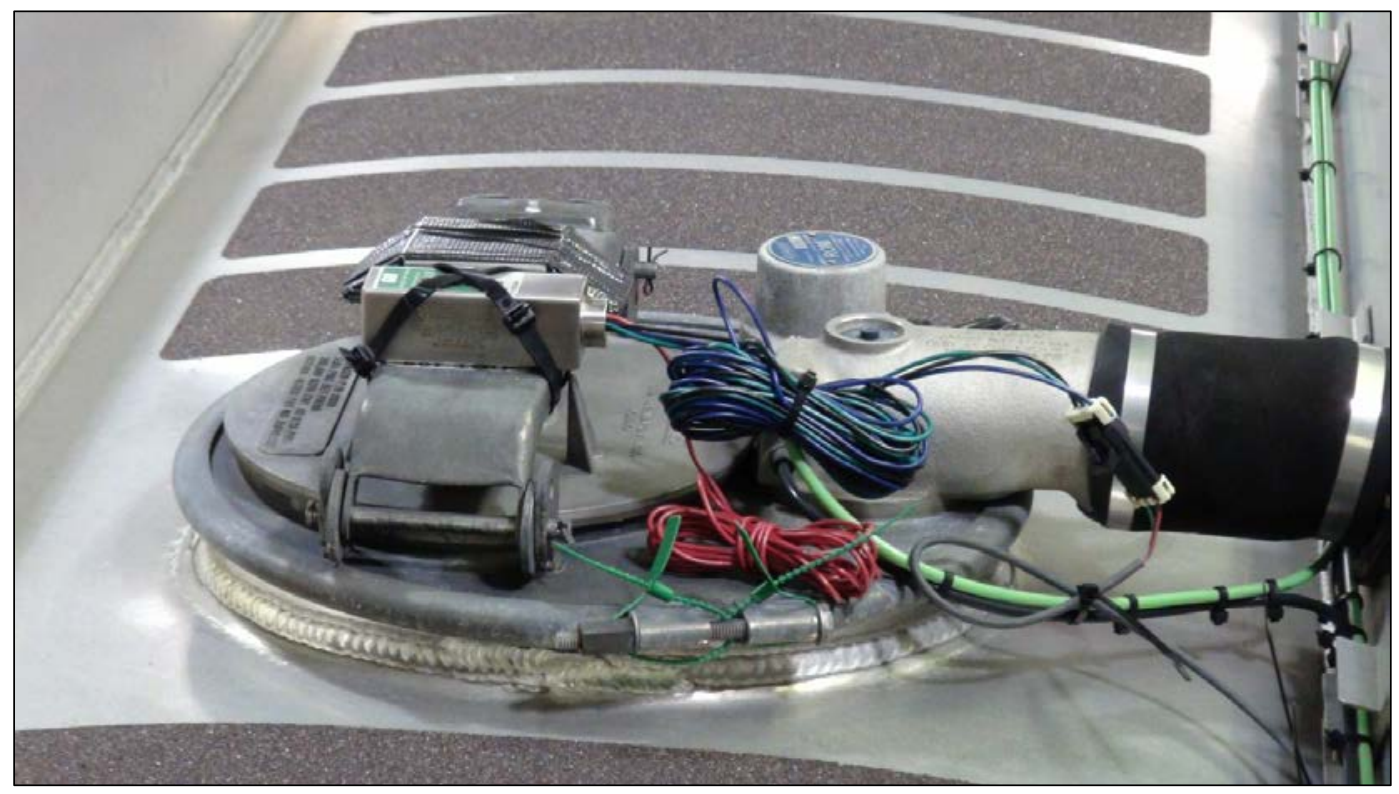

Fig. 89. Instrumented hatch located on top of tanker.

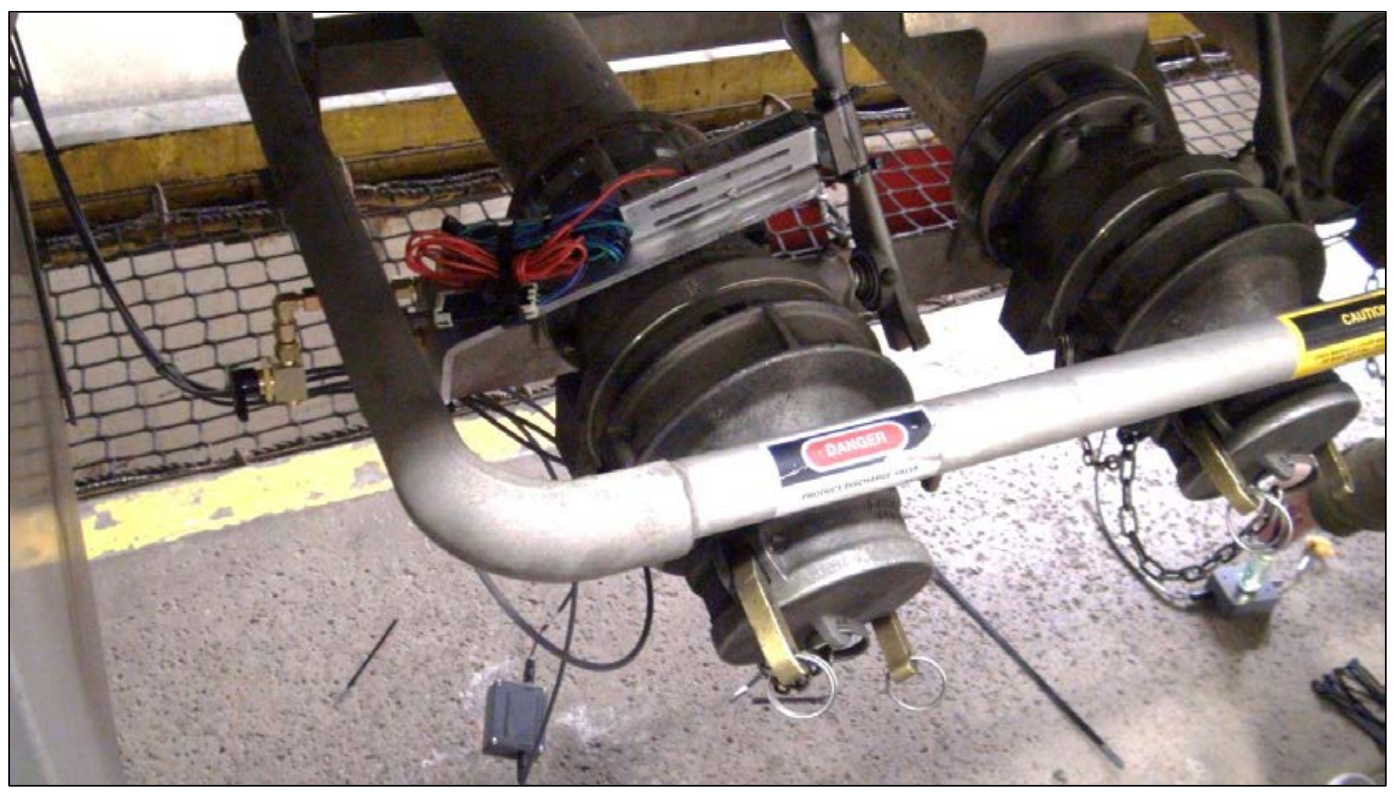

Fig. 90. Instrumented valve on tanker.

The ORNL DAS was installed in the tractor, along with a GPS antenna (Fig. 91). Connections were made to the vehicle's power and data bus, where the data from the T2TCU would be posted.

The tractor-based Air-Weigh ComLink and display had to be replaced with a J1939 compatible unit. This equipment was on hand at the time.

Following the pre-test loading of fuel in the instrumented compartments, minor leaks were identified in the flange connections of the sight glasses as well as in the connections to the pressure transducers. These connections were sealed by a TRC mechanic and retested to confirm there were no additional leaks. 


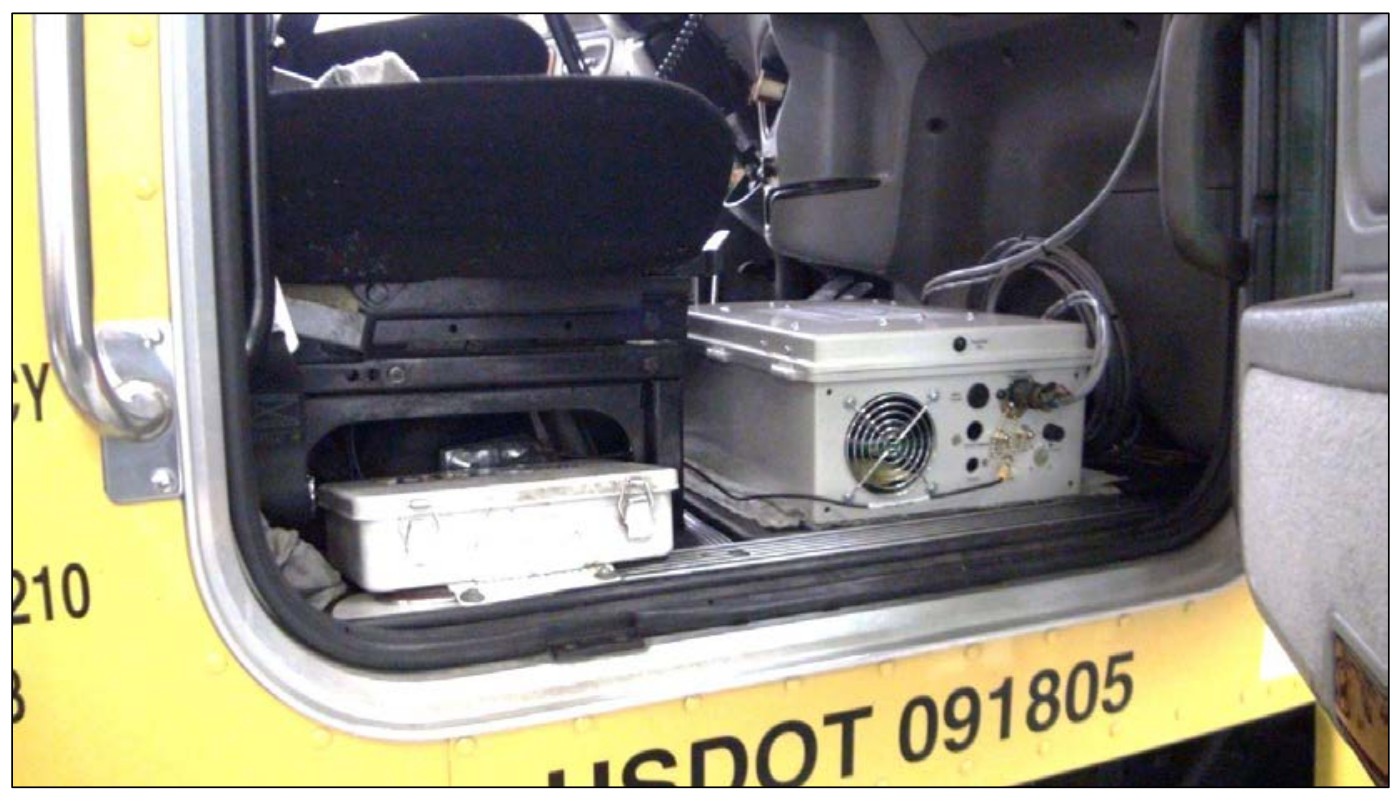

Fig. 91. DAS installed in test vehicle.

At the conclusion of the upfit, the Pilot Travel Centers vehicle was weighed in a near-empty state using the TRC pit scale, and then again with the addition of 1,000 gal of fuel in order to calibrate the tractor and trailer Air-Weigh on-board weighing systems.

The sensors and DAS were tested and confirmed to be functional. Although a connection to the ORNL computers was not possible due to ORNL network being closed, a work-around was facilitated with another researcher working from home on his personal Internet connection.

During the vehicle upfit, TRC personnel mixed the marker into the additional 350 gal of fuel needed for testing, combining this fuel with the fuel marked in the previous fuel loop testing. Dry aliquots were transported and mixed into multi-gallon volumes of diesel for the test track trials, and this approach proved far more reliable in regard to full, fast dissolution of the dry SiNaph marker into the large volume, turbulent flow containers than the method employed in the earlier fuel loop testing. Steps were taken to avoid the incomplete mixing issues experienced in the fuel loop testing. To prevent the issue of highly concentrated marked fuel getting trapped in dead sections of the hosing, the test apparatus was not used in the initial mixing of the fuel; instead, hosing of a diameter ( 2 in.) consistent with the piping on the mixing truck was used. To alleviate the issues caused by splashing in the tank, the mixing was performed over several hours and with the fuel 350 gal, reducing the area within the tank for splashed fuel and allowing the splashed fuel to drain into fuel mixture. After several hours of mixing to ensure a complete mix, the fuel test loop used in March testing at TRC was set up and configured to allow ORNL personnel to verify the complete mixing based on the stable measurements of the concentration observed during the period following the mix.

At the beginning of the first day set aside for testing, the FTE monitoring system was found to be working erratically. It was suggested after consultation with Air-Weigh that there might be data collisions on the J560 connector between the tractor and trailer due to the fact that several vehicle systems use this line for communications. The team decided to run a dedicated power line for the T2TCU and the Air-Weigh ComLink. This action solved the erratic behavior of the system, and scenario testing began at noon on Monday, April 18, 2011. 
The Analysis Team members began testing by conducting an initial assessment of the "control center" room (TRC-provided conference room) to ensure internet was accessible to the team. Additional checking of the computer equipment was performed at the hotel room the evening before the official test. Due to the shutdown of the ORNL network, the originally designated data server located at ORNL could not be accessed. In response, the data retrieval program was modified to store files locally, and this new version was installed and configured on the standalone desk top computer that was brought to Ohio. This was an unexpected complication, but with extensive communication between the Evidential Reasoning and Analysis Teams, a temporary on-site data collection solution was effectively set up by midnight prior to testing. The system was ready for field testing the following morning.

\subsubsection{Testing Activities}

The testing (April 18-19, 2011) was designed around a few representative scenarios from which the evidential reasoning could identify several different types of FTE.

- Nominal-legal movement of taxed and untaxed fuel (baseline)

- Alternate Nominal-legal movement of untaxed fuel (additional scenario for sensor verification)

- Jurisdiction Crossing-illegal diversion of taxed fuel

- Theft—portion of load of taxed fuel diverted/stolen en route

- Blending I-Dilution of marked fuel with an equal amount of unmarked fuel en route

- Blending II—mixing of a small amount of simulated hazardous waste with marked fuel en route

- Blending III—marked fuel mixed with unmarked fuel en route.

A TRC fuel truck was used to simulate both the fuel rack and the fuel drop (convenience store or other). Figure 92 shows a typical connection used during the testing. In this image, the transfer is taking place at the Fuel Plaza, one of four locations designated by TRC to simulate various loading and offloading locations.

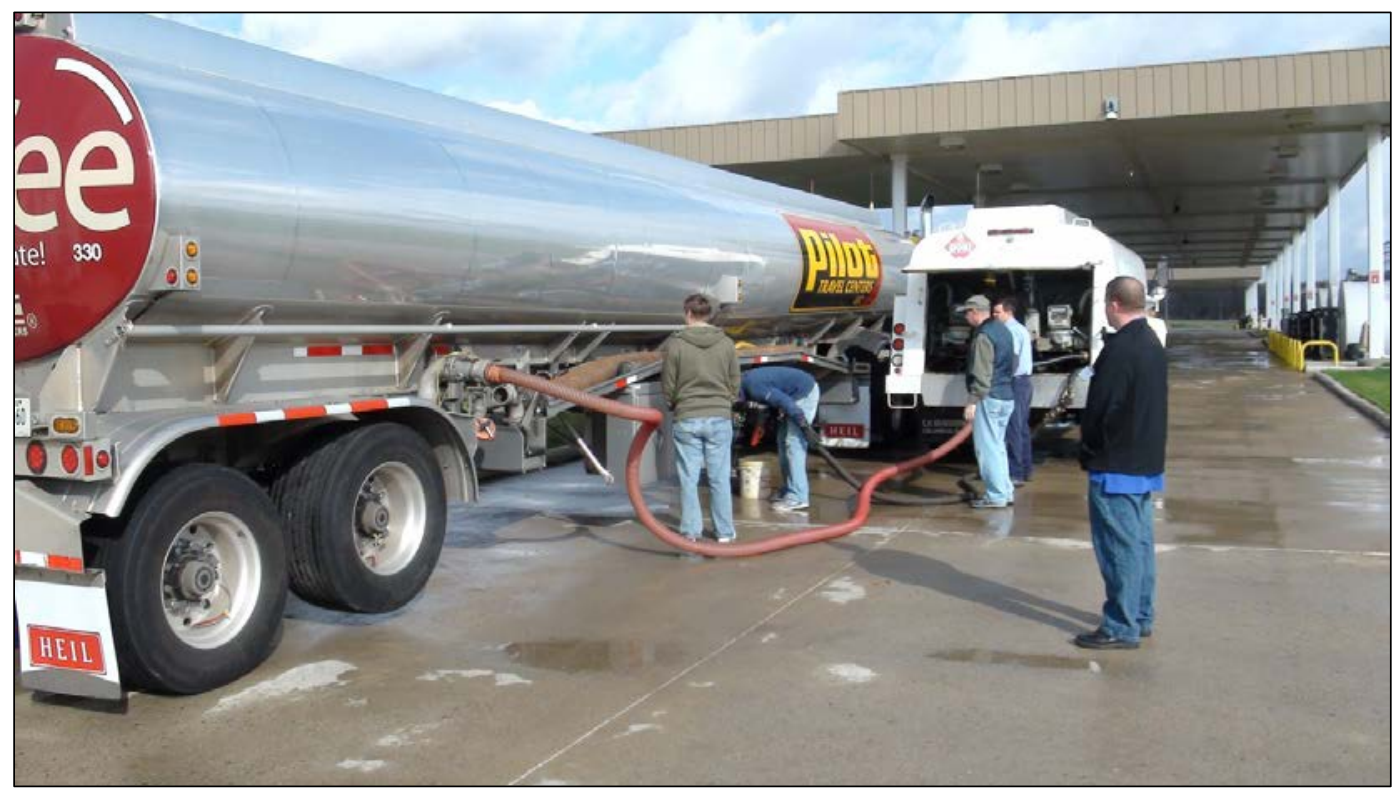

Fig. 92. Test vehicle connected to TRC tanker for fuel transfer.

All computer systems were set up in the control center on Monday morning, April 18, 2011. The data retrieval algorithm was tested again and no issues found. Some of the parameters in the evidential reasoning program were adjusted during the setup based on new information provided by the Sensor team 
such as marker reading error bound and fueling rate. The FHWA project sponsors visited the control center for a short briefing on the reasoning logic and a short demo of GPS data tracking/monitoring on the GIS system, using the data collected from the pretesting exercise.

Figure 93 shows the routes taken by the Pilot tanker while testing at TRC. Communication issues on the DAS occurred intermittently throughout the day, primarily due to missing cellular signals in some zones. However, a local copy of the data was stored on the DAS and retrieved for post-processing at the conclusion of each day's testing to fill in any data gaps in the real-time data files. Minor issues with instrumentation were seen during testing and identified in the post-processed data. These are described in the greater detail in Sect. 7.

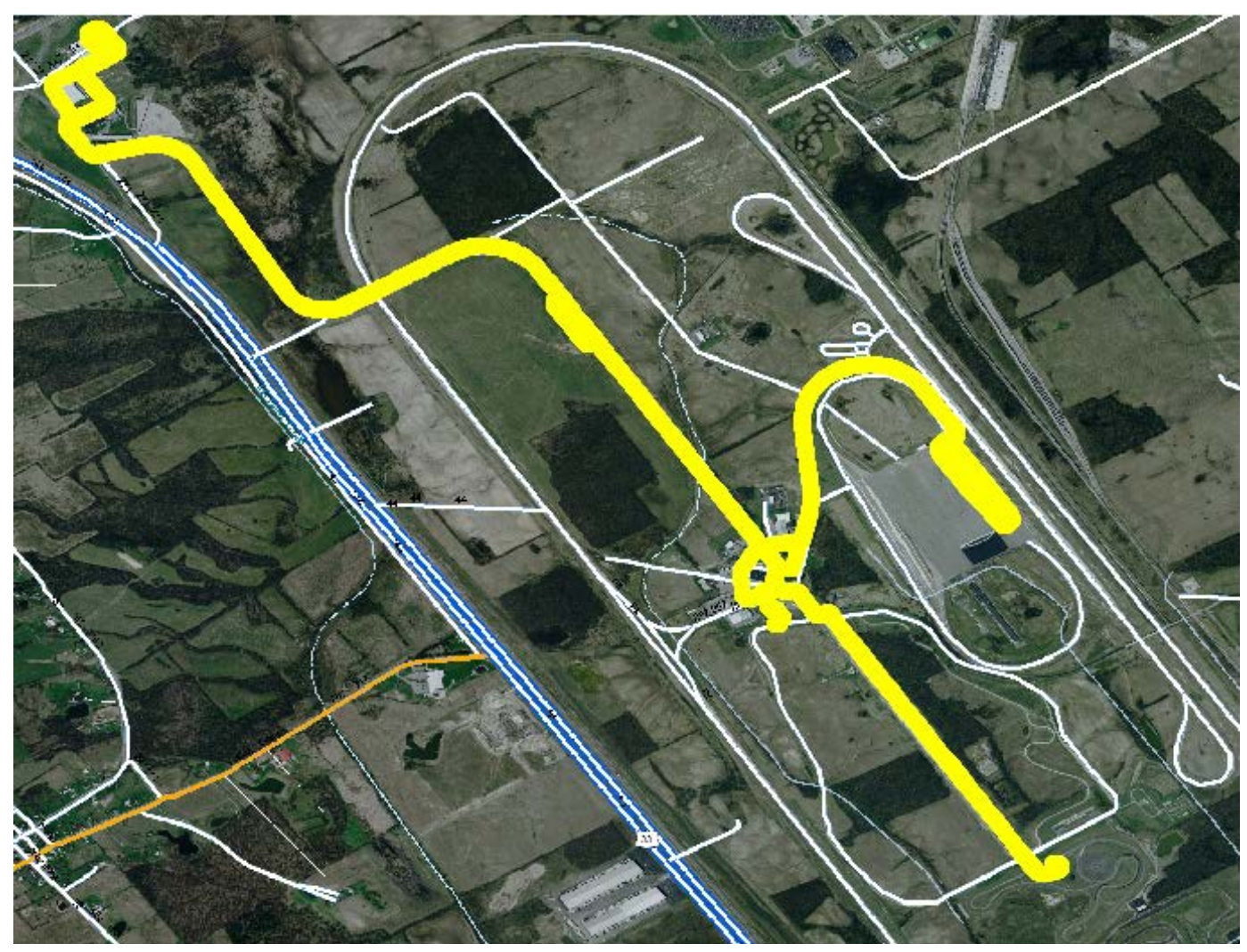

Fig. 93. Tanker routes at TRC during second day of field test.

Communication issues with the data retrieval system occurred throughout the day, mainly due to cellular dead zones within the TRC site where the tanker was traveling. The Evidential Reasoning Team relied on phone communications with the Communications Team (both on site with the tanker and offsite in Knoxville, Tennessee). The communications with on-site researcher, however, were much limited due to the dead-zone issue mentioned previously.

During the times that the system was communicating properly, a data file from each 5 min transmission was successfully received, converted, and stored into the database that fed the GIS system, as soon as it appeared in the designated file folder on the "server" (i.e., the desktop computer or the temporary data retrieval system). Due to data gaps in the signal transmissions, however, only limited near-real-time analysis (analysis on the files received every $5 \mathrm{~min}$ ) could be conducted. The full set of data was obtained from the on-board system on Monday evening, which provided better data with much less missing signals. 
All post-processed data files from the first day of testing were successfully converted into a readable format for the GIS system. Unfortunately, data from the loading process of the first scenario was missing from the data set, likely due to a power issue the Communications Team encountered during the first part of the first day of testing. Certain "disappearing" of signals could clearly be observed from the data collected. With the exception of a few moments at the beginning of the day, the testing process was very similar to the first day of testing. Because the team was not aware that the TRC internet has an overnight time-out policy (probably due to guest login), there were some confusions as to why the DAS would not communicate properly on Tuesday morning. Communication issues were encountered the second day of testing as well.

One of the greatest challenges encountered during the second day of field testing was the weather. Adverse weather conditions further added to the already troublesome communication issues. Along with the DAS connectivity issue, one of the valve sensors gave incorrect values for a portion of the test, likely due to a mounting issue.

\subsubsection{Results and Data Analysis}

Due to the mounting solutions employed for the marker concentration and fuel level sensors, it was determined that readings from all sensors would only be "valid"-for the purpose of evidential reasoning - during the periods that the valve was open and fuel was flowing through the instrumented sight glass. In fact, due to the installation locations of sensor devices in the sight glass, the marker concentration and fuel level sensors are not capable of obtaining fuel activities during the time period prior to the valve opening or the time period after the valve closed. Thus, all analyses were conducted using data collected during time periods that the valves are opened.

Due to the mounting configuration, the fuel level data typically had a higher level of noise in its readings, particularly when the valve was first opened. The noise level in fuel level data was clearly higher when the fuel was loaded into the compartments; that is, data were smoother when the fuel was offloaded from the tanker. Similarly, marker concentration data also had a higher noise under the fuel intake process than when the fuel was being offloaded. The noise in the marker sensor data for the opening and closing parts (when valve was first opened and when valve was about to close) seemed to also be higher than those in the mid-stream for the marker sensors.

Activities from each of the test scenarios are presented in the following section. Data gathered from the loading and offloading processes, by compartments, are presented in separate figures. The compartments are referred as "valve 1" and "valve 4" in the charts shown. Note that certain default (preset) values are used when sensor signals are "missing." For fuel level, the default was 35, while fuel marker was set at 1.0. Some of these can be observed in the figures that follow.

\subsubsection{Nominal scenario}

The initial trip was designed to verify the functionality of all the sensors and evidential reasoning system through implementing a legal scenario. In this scenario 500 gal marked fuel and 500 gal unmarked fuel were loaded onto the tanker at the Fuel Plaza. The marked fuel was then offloaded at Building C, and the unmarked fuel offloaded at the WRC Pad.

\section{Loading period}

As pointed out earlier in this report, data from this time period were not collected due to a power issue associated with a drained truck battery. This issue was corrected, and similar problems were prevented during the remainder of the testing by leaving the truck running at all times to avoid draining the battery. 


\section{Off-loading period}

Data from the offloading process for both compartments were analyzed for scenario one. Based on the compartment 1 reading obtained from sensors (Fig. 94), measurements at the conclusion of offloading that would be used for evidential reasoning algorithm were

- Type of stop: scheduled,

- Marker concentration 0.5, and

- Final fuel level 0.0.

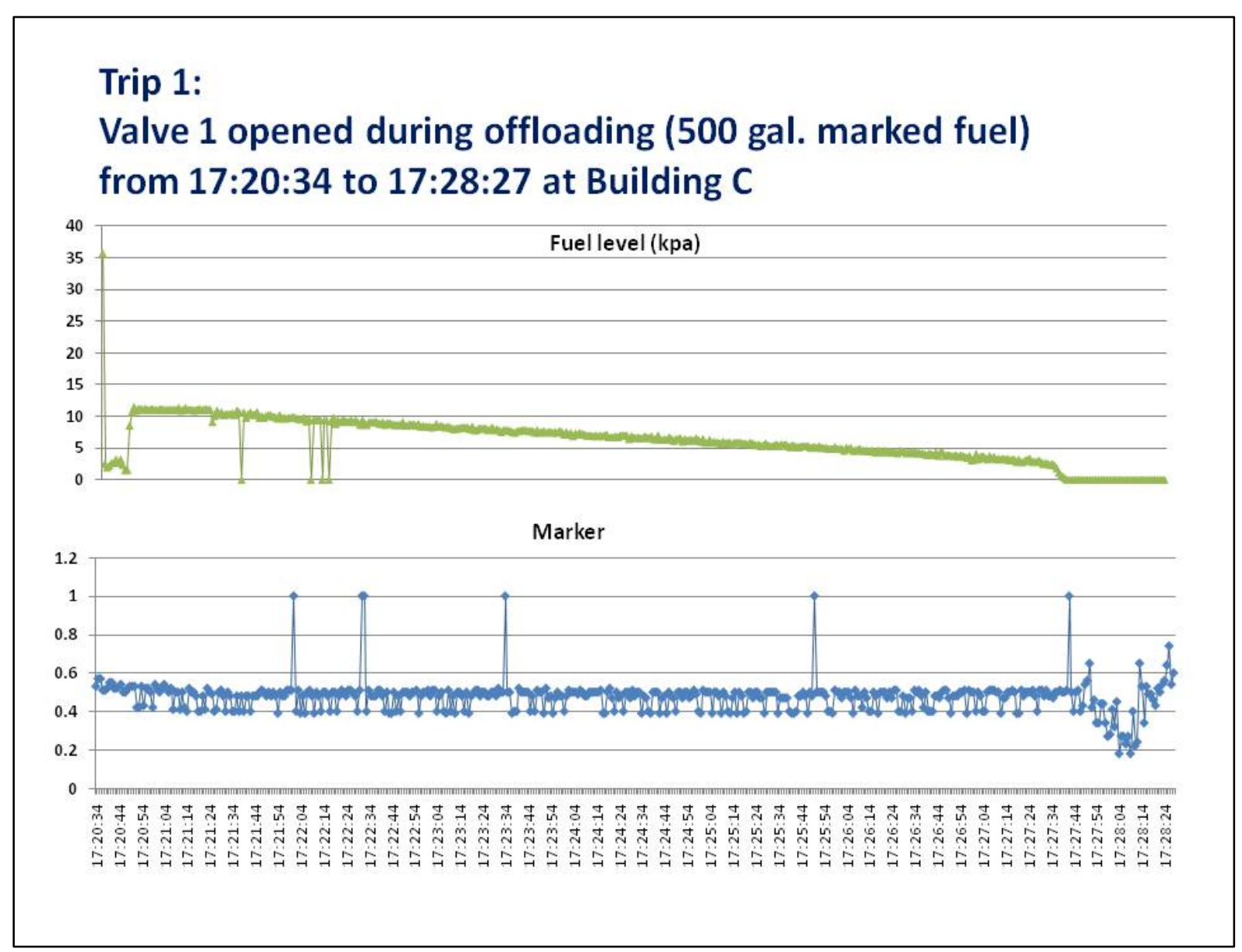

Fig. 94. Activity from offloading of compartment 1 under Scenario 1.

Based on the compartment 4 reading obtained from sensors (Fig. 95), measurements at offloading that would be used for evidential reasoning algorithm were

- Type of stop: scheduled,

- Marker concentration 0.2, and

- Fuel level 0.0 .

\section{Warning level estimates}

An alert was not expected for this scenario, but since measurements from loading period were not available, no warning score could be computed for either compartment 1 or compartment 4 . 
Trip 1:

Valve 4 opened during offloading (500 gal. unmarked fuel) from 17:50:58 to 17:58:25 at WRC
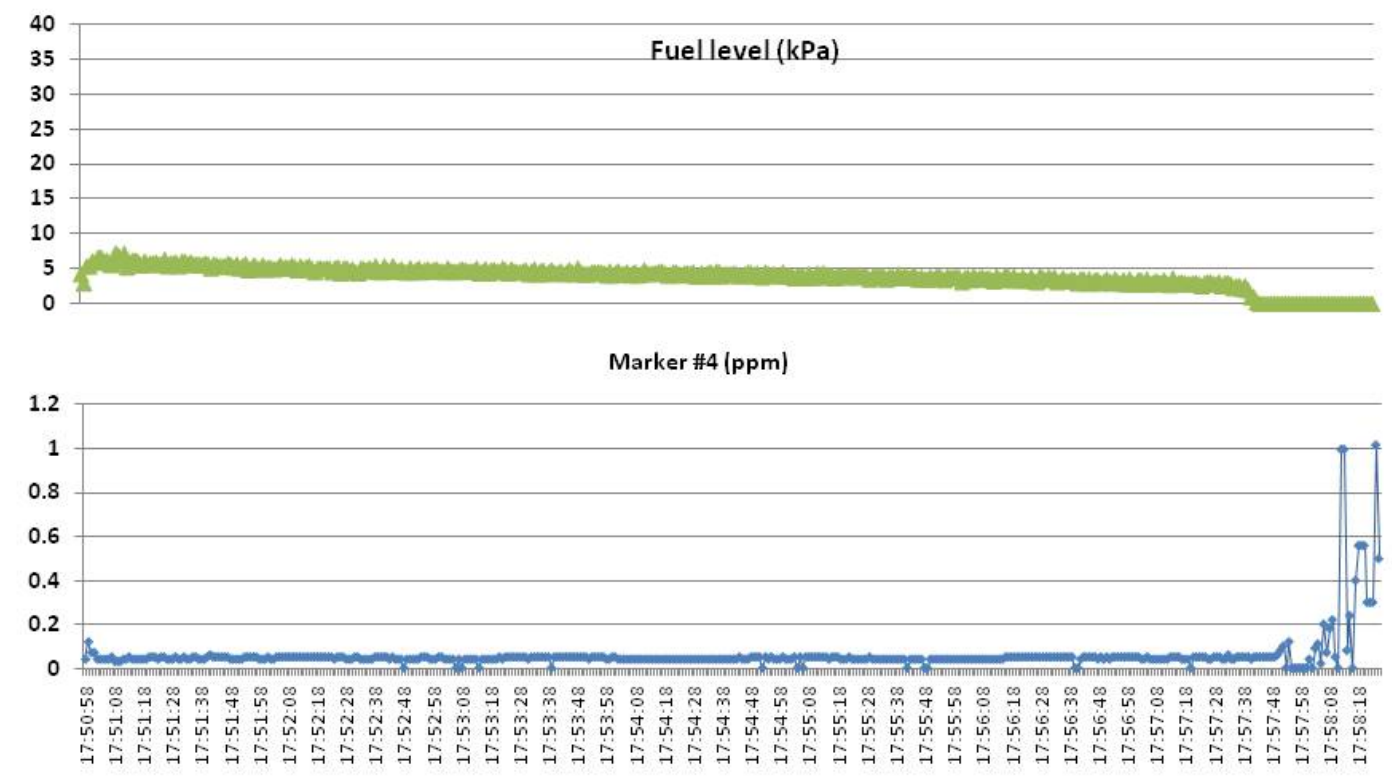

Fig. 95. Activities from offloading of compartment 4 under Scenario 1.

\subsubsection{Jurisdiction crossing and alternate nominal scenarios}

The next trip was designed to incorporate "illegal" actions to begin testing the evidential reasoning system's ability to recognize suspicious deviations from the "assigned" route. The officially assigned route was to begin with the loading of 300 gal unmarked fuel and 400 gal marked fuel at the WRC Pad. The unmarked fuel was then to be offloaded at the SE VDA, and the marked fuel at the Fuel Plaza. In keeping with the "official" route, the marked fuel was offloaded as prescribed. However, delivery in a different jurisdiction was simulated when the marked fuel was offloaded at Building $\mathrm{C}$ instead.

\section{Loading period}

Data from the loading process for both compartments were captured and analyzed for the second trip of the field test. As mentioned previously, the noise level in fuel level data was relatively high when the fuel was being loaded into the compartment. The noise level for the marker concentration data typically was also higher at the beginning of fueling process. Based on the compartment 1 reading obtained from sensors (Fig. 96), measurements during the loading of compartment 1 were

- Marker concentration 0.065 (the fuel was unmarked, i.e., ideally it should have been 0.00) and

- $\quad$ Fuel level 8.680. 


\section{Trip 2:}

\section{Valve 1 opened during loading ( 300 gal. unmarked fuel)}

from 18:01:55 to 18:08:08 at WRC

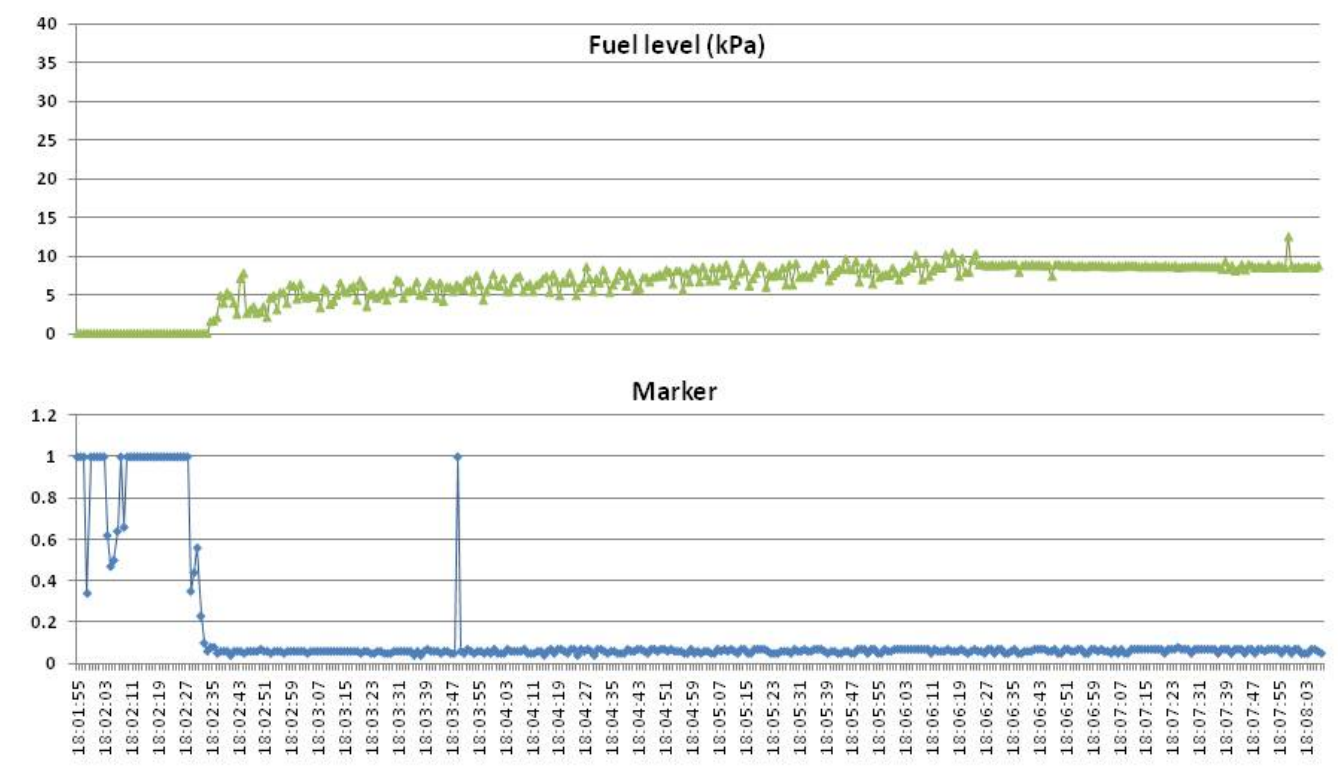

Fig. 96. Activities from loading of compartment 1 under Scenario 2.

Similar observations on data noises could be seen from the loading of compartment 4 (Fig. 97). The measurements at loading of compartment 4 were

- Marker concentration 0.825 and

- Fuel level 12.950.

\section{Off-loading period}

Again, data noise level in the fuel pressure was much lower for the offloading process. Due to issues with the marker sensor originally installed on this compartment, a replacement with a different operating scale was installed before the compartment of fuel was offloaded. Noise level for the marker concentration was much worse toward the end of offloading, which seemed to be corresponding to the "empty" fuel tanks. Based on the compartment 1 reading obtained from sensors (Fig. 98), measurements at offloading that would be used for evidential reasoning algorithm were

- Type of stop: scheduled,

- Marker concentration 0.07, and

- $\quad$ Fuel level 0.280 . 


\section{Trip 2:}

Valve 4 opened during loading (400 gal. marked fuel) from 18:16:02 to 18:22:15 at WRC

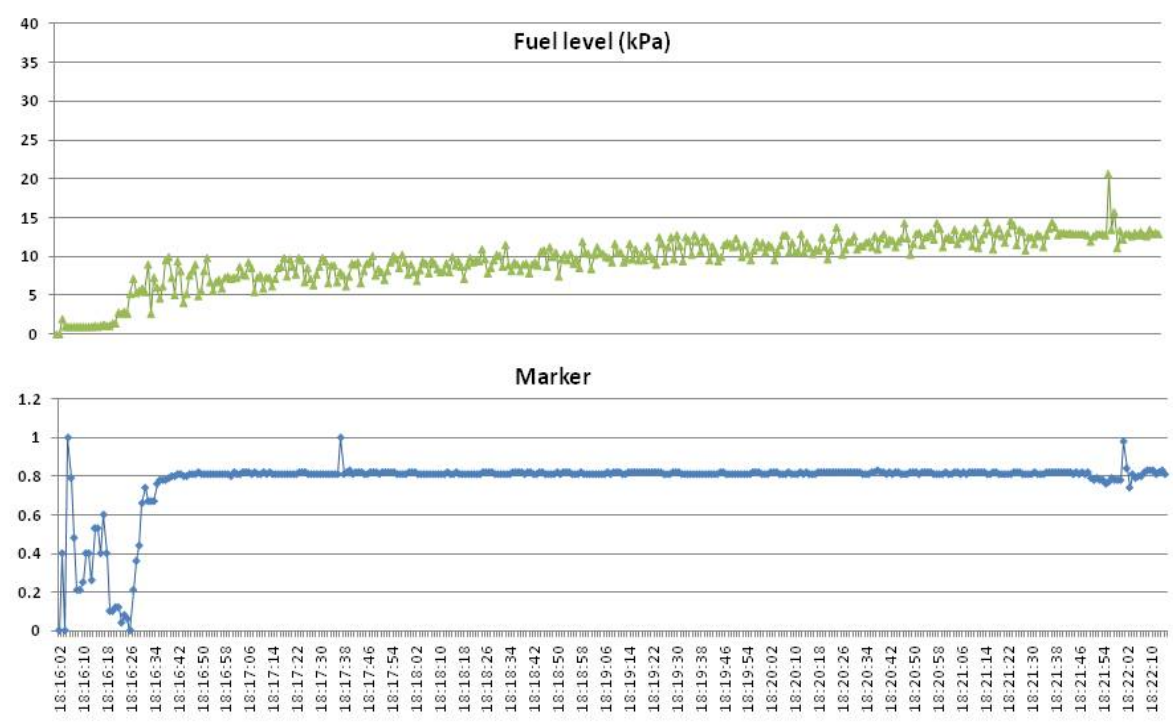

Fig. 97. Activities from loading of compartment 4 under Scenario 2.

Trip 2:

Valve 1 opened during offloading (300 gal. unmarked fuel) from $18: 41: 10$ to $18: 46: 11$ at SE VDA

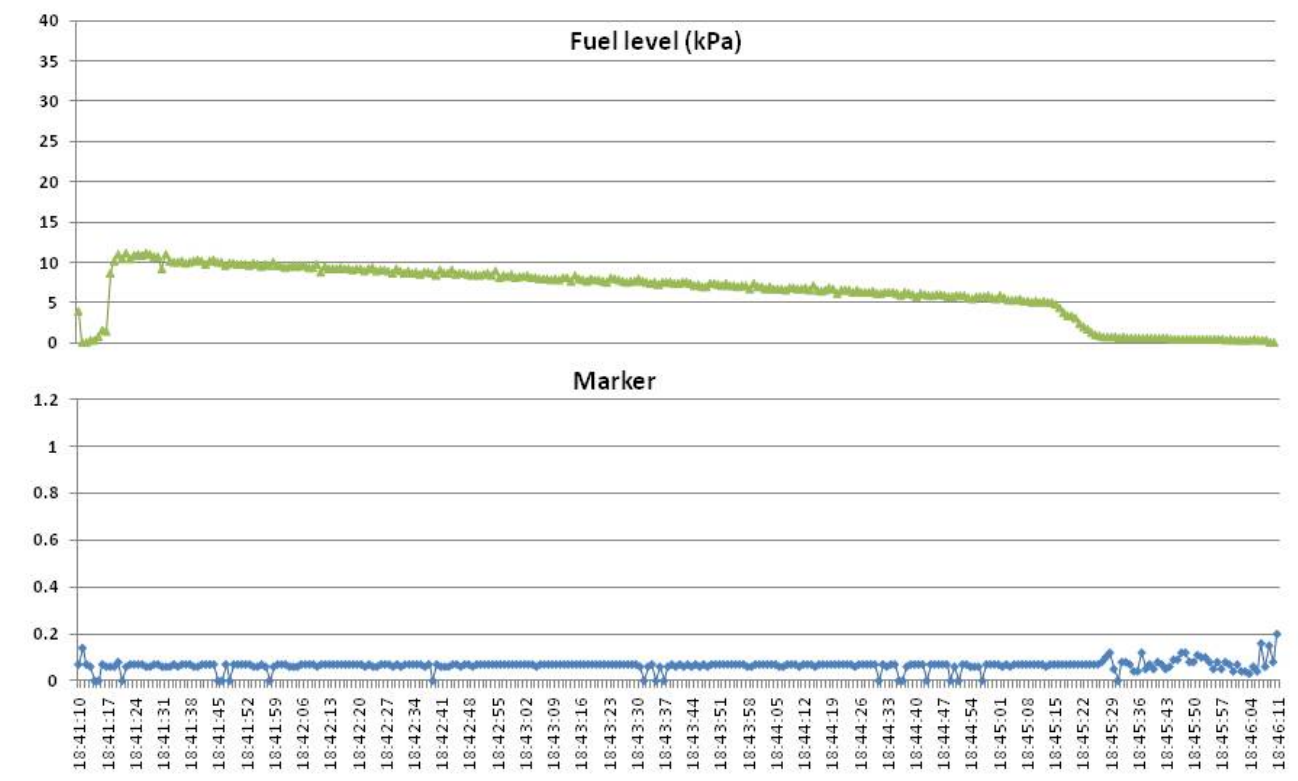

Fig. 98. Activities from offloading compartment 1 under Scenario 2. 


\section{Warning level estimates after offloading compartment 1}

The warning level based on evidential reasoning algorithm returned a value of 58\%, which was high for a "legal” case. This false alarm was mainly caused by two factors:

1. The change of marker concentration from 0.065 to 0.07 is about $8 \%$, which was higher than the preset threshold of $5 \%$ for error bound. Furthermore, it was also expected that the marker concentration value for "unmarked" fuel would be near 0.00 . A reading of 0.07 was thus determined as "marked" by this interpretation. This reflects the larger than the noise level expected by the algorithm in the marker concentration data generated from the optical sensor.

2. The fuel level of 0.280 was outside the threshold set for "empty" tank. Similar to above, this was also due to unexpected level of noise in the data collected from the sensor.

Again, noise could be seen in fuel level measures at the beginning of offloading compartment 4 (Fig. 99), as well as those toward the end of marker sensor readings. As pointed out previously, the fluctuating marker sensor measures appeared to be associated with the drop of fuel level to the empty state (zero). Measurements from the offloading of compartment 4 used for the evidential reasoning were

- Type of stop: unscheduled,

- Marker concentration 0.400, and

- $\quad$ Fuel level 0.000 .

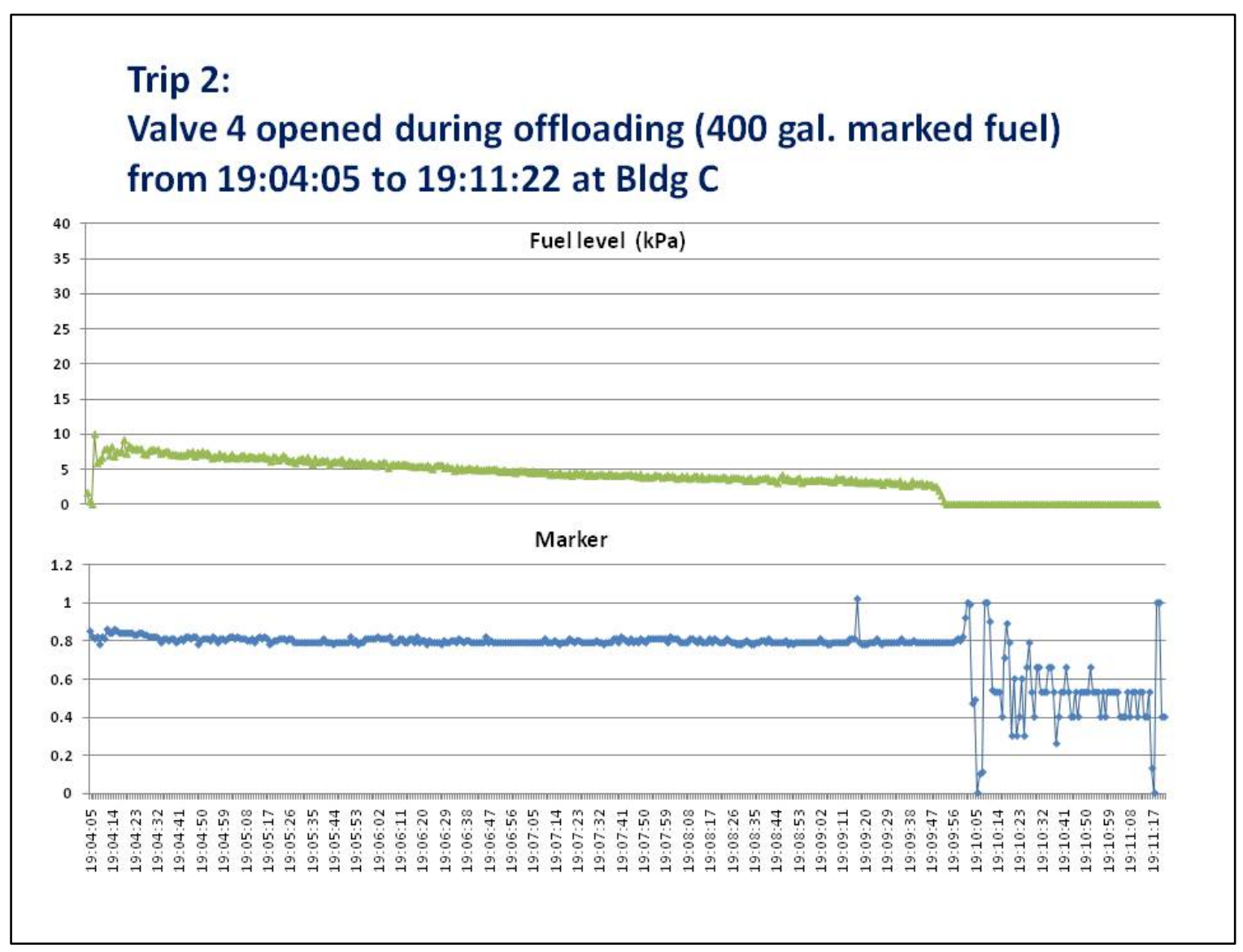

Fig. 99. Activities from offloading compartment 4 under Scenario 2. 


\section{Warning level estimates after offloading compartment 4}

The warning level based on information collected from sensors and evaluated by the evidential reasoning algorithm returned a high value of $84 \%$. Two factors caused the alert: an unscheduled stop where the valve is opened and a large change (a 52\% decrease) in the marker concentration (dropping from the reading of 0.825 at loading to a value of 0.400 at offloading). Thus the crossing jurisdiction case was successfully detected, although the marker sensor should not have reported a change in concentration for this scenario.

\subsubsection{Theft scenario}

"Legal” activities for this route would have involved the transfer of 400 gal marked fuel from Building C to the WRC Pad. However, the test vehicle stopped at the SE VDA after loading to offload 50 gal of its marked fuel payload, thereby simulating theft in transit. Only 350 gal of fuel remained to be delivered to the WRC Pad. Only one compartment was used in this scenario.

\section{Loading period}

This scenario only utilized one compartment (compartment 1), and the total amount involved was 400 gal of marked fuel. Similar to previous scenarios, noise levels in fuel level data were relatively high when the fuel is being loaded into the compartment. Noise on the marker concentration data was also higher at the beginning of fueling process, with a few missing signals (shown as 1.0) occasionally. Based on the compartment 1 reading obtained from sensors (Fig. 100), measurements at loading of compartment 1 were

- Marker concentration 0.575 and

- Fuel level 10.920.

\section{Off-loading periods}

\section{First stop}

This activity offloaded 50 gal from the tanker, which not only was a very small amount but also a very short process that only lasted about 1.5 min (from valve opened to valve closed). Again, the noise level in the fuel level data was much lower for this offloading process. The marker concentration data did not show significant noise level changes (besides a missing signal), when compared to the noise previously observed. Based on the compartment 1 reading obtained from sensors (Fig. 101), measurements for this stop resulted in the following values:

- Type of stop: unscheduled,

- Marker concentration 0.584, and

- Fuel level 6.650.

\section{Second stop}

This is the "scheduled" delivery destination, where the remaining 350 gal were offloaded from the tanker. As discussed previously, noise in the fuel level signal was low except at the beginning of the offloading process. The marker concentration data again showed a significant increase in noise level when the fuel level dropped to zero at the end of the offloading process. Based on reading obtained from sensors (Fig. 102), measurements for this stop are

- Scheduled stop,

- Marker concentration 0.728, and

- Fuel level 0.000. 
Trip 3:

Valve 1 opened during loading (400 gal. marked fuel) from 19:21:10 to 19:27:46 on 4/18/11 at Bldg C

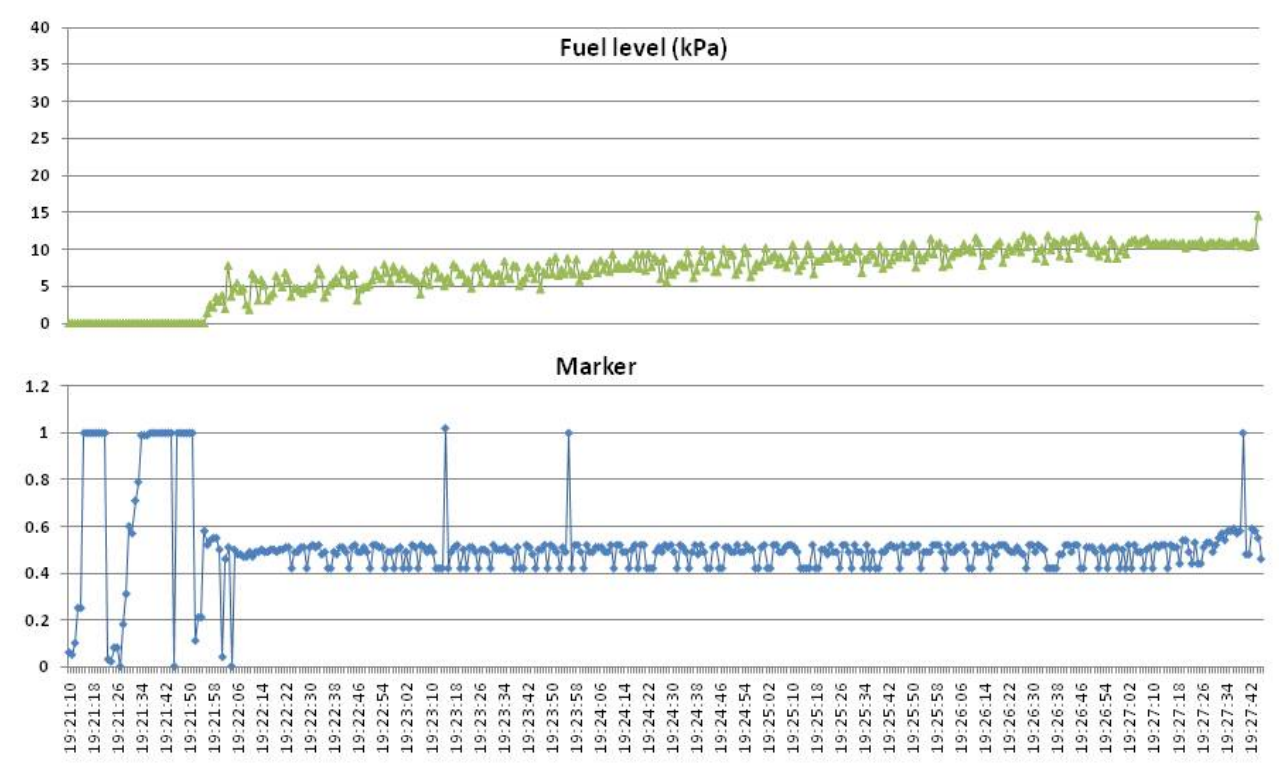

Fig. 100. Activities from loading of compartment 1 under Scenario 3.

\section{Trip 3:}

Valve 1 opened during offloading ( 50 gal. fuel) from 13:45:13 to 13:46:36 on 4/19/11 at SE VDA
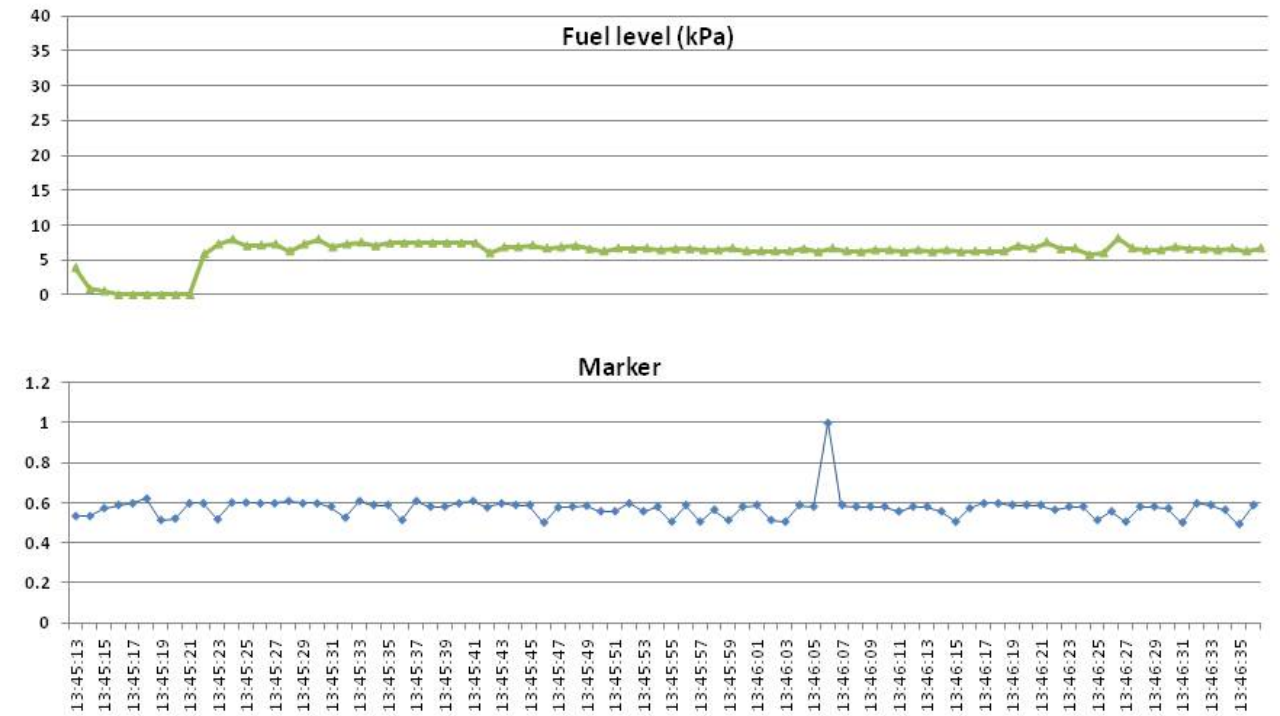

Fig. 101. Offloading 50 gal at an unscheduled location under Scenario 3. 


\section{Trip 3:}

\section{Valve 1 opened during offloading (350 gal. fuel)}

from 14:05:33 to $14: 11: 22$ on $4 / 19 / 11$ at WRC

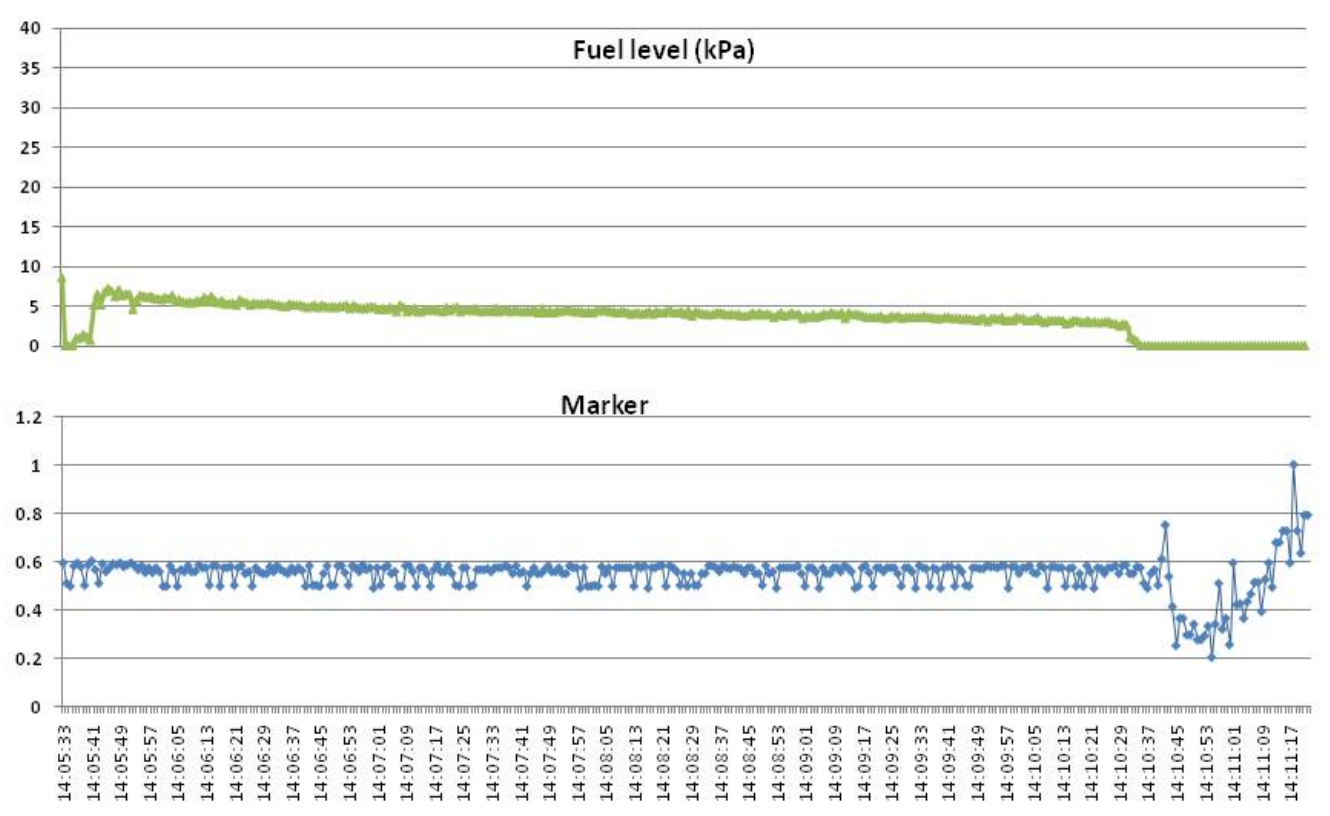

Fig. 102. Activities measured from offloading at destination under Scenario 3.

\section{Warning level estimates}

\section{Unscheduled stop}

As indicated previously, due to design limitations regarding where the sensors could be installed, fuel characteristic information could only be measured when it flowed through the valve. Thus, in this case, the small amount ( 50 gal) of fuel moving through the sensors could only be "seen" for less than 1.5 min. This short time frame did not prevent the sensors from performing their functions. The optical sensor successfully read what was needed to determine that the fuel marker concentration was acceptable, 1.5\% difference from the "base" measured at loading, which was within the error bound.

The reading from the fuel level sensor, which was limited to measure what was flowing through at that time period, however, could not really be used for comparison with the "base" value. The major alarming activity for this stop then was the unscheduled stop. The estimated warning level for this stop was $48 \%$, not a high value but one that could be an initial warning pending on further confirmation from other actions (i.e., waiting on results from next activity).

\section{Final destination stop}

The final warning level estimated for this scenario was 100\%. The final measure for the fuel marker concentration was higher than normal mainly due to the large variation at the end when the fuel was dropped to empty. In the future, modification of avoiding reading marker measurements from the period when the fuel level is down to zero should be implemented. In this case, both marker and fuel level showed variations above the maximum error bound set for the algorithm. It reflects a supposedly very 
likely anomaly, even in the measurement itself or due to actual abnormal operations. This might be a false positive warning, since such high warning was not expected. This might have indicated that the proposed method of anomaly detection failed, or it might be inappropriate, or the data quality caused "confusion" in the algorithm. Further investigation and improvement is, thus, needed.

\subsubsection{Blending Scenario 1}

This scenario also only utilized one compartment (compartment 4). The "assigned" scenario called for the test vehicle to deliver 300 gal of marked fuel from the WRC Pad to Building C. Instead, the test vehicle loaded only 150 gal of marked fuel at the WRC pad, and stopped in route at the Fuel Plaza to add 150 gal of unmarked fuel, thus simulating the blending of taxed and untaxed fuel to be "passed off" as taxed. The mixed fuel was delivered to Building C.

\section{Loading period}

\section{First loading}

Same as those observed previously, the noise level in the fuel level data was relatively high when the fuel was being loaded into the compartment. Noise in the marker concentration data was also higher at the beginning of the fueling process. Since only 150 gal were loaded, it took a little more than 3 min for the entire process, from valve opened to valve closed. Based on the compartment 4 readings obtained from sensors (Fig. 103), measurements at this loading were

- Marker concentration 0.936 and

- $\quad$ Fuel level 7.000.

\section{Second loading (first stop after initial loading)}

Although this was another fuel loading, it was not included in the vehicle's "legal” route and was therefore considered to be an unscheduled stop. The noise level in the fuel level data was again high during the loading process. Noise in the marker concentration data was higher at the beginning of the fueling process but dropped dramatically when it started "seeing" the unmarked fuel which was loaded at this stop. This additional 150 gal also took about $3 \mathrm{~min}$ to load from the time the valve was opened to the time it was closed. Based on the readings obtained from the sensors (Fig. 104), measurements for this loading were

- Type of stop: unscheduled,

- Marker concentration 0.096, and

- $\quad$ Fuel level 7.560.

\section{Off-loading periods}

This activity offloaded 300 gal as scheduled from the tanker, which should have been all marked fuel (had the vehicle's activities been confined to the "legal" scenario). As shown in Fig. 105, similar noise levels existed as those that had been observed from other cases. The fuel level, however, did not completely drop to the zero level at the end of the offloading. Measurements for this stop resulted in the following values:

- Scheduled stop,

- Marker concentration 0.354, and

- Fuel level 0.840 . 
Trip 4:

Valve 4 opened during loading (150 gal. marked fuel) from 14:16:03 to $14: 19: 21$ on $4 / 19 / 11$ at WRC

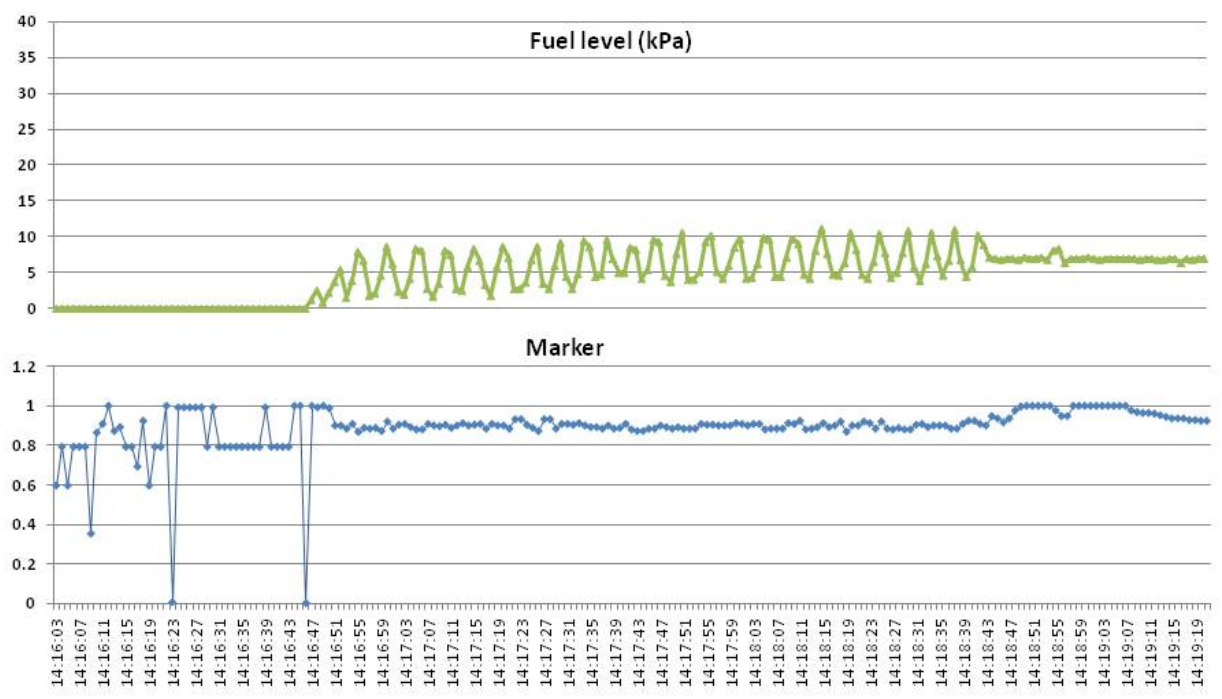

Fig. 103. Activities of loading first 150 gal under Scenario 4.

\section{Trip 4:}

Valve 4 opened during loading (150 gal. unmarked fuel) from 14:38:11 to $14: 41: 07$ on 4/19/11 at Fuel Plaza

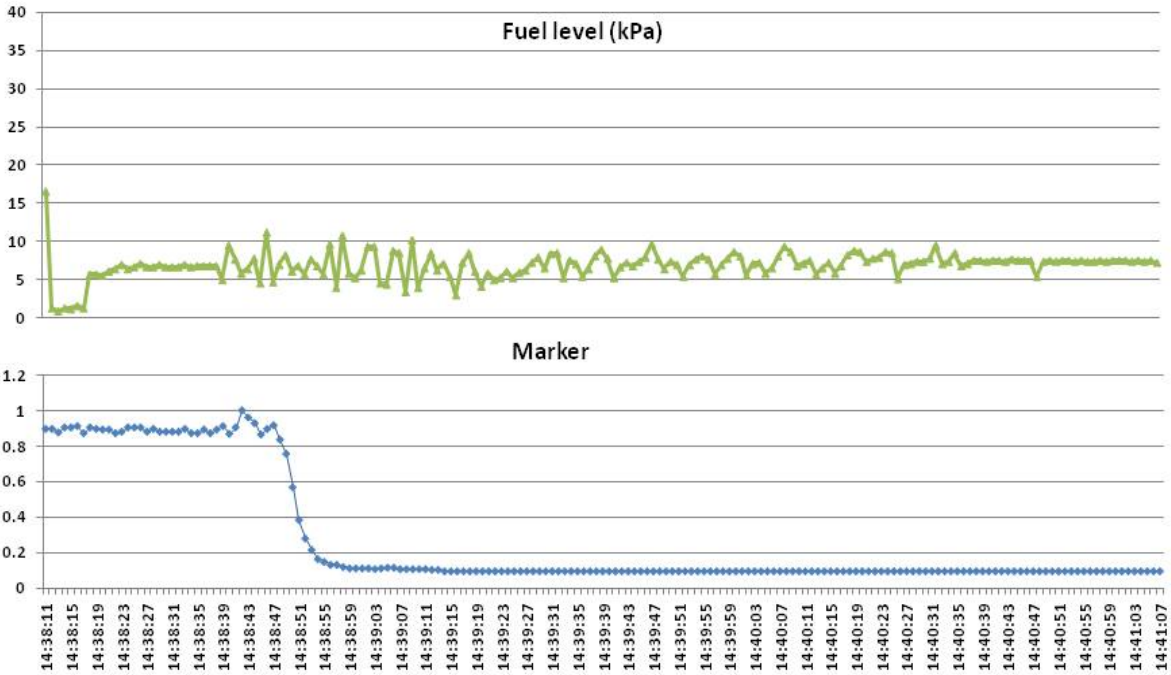

Fig. 104. Activity from adding 150 gal unmarked fuel under Scenario 4. 


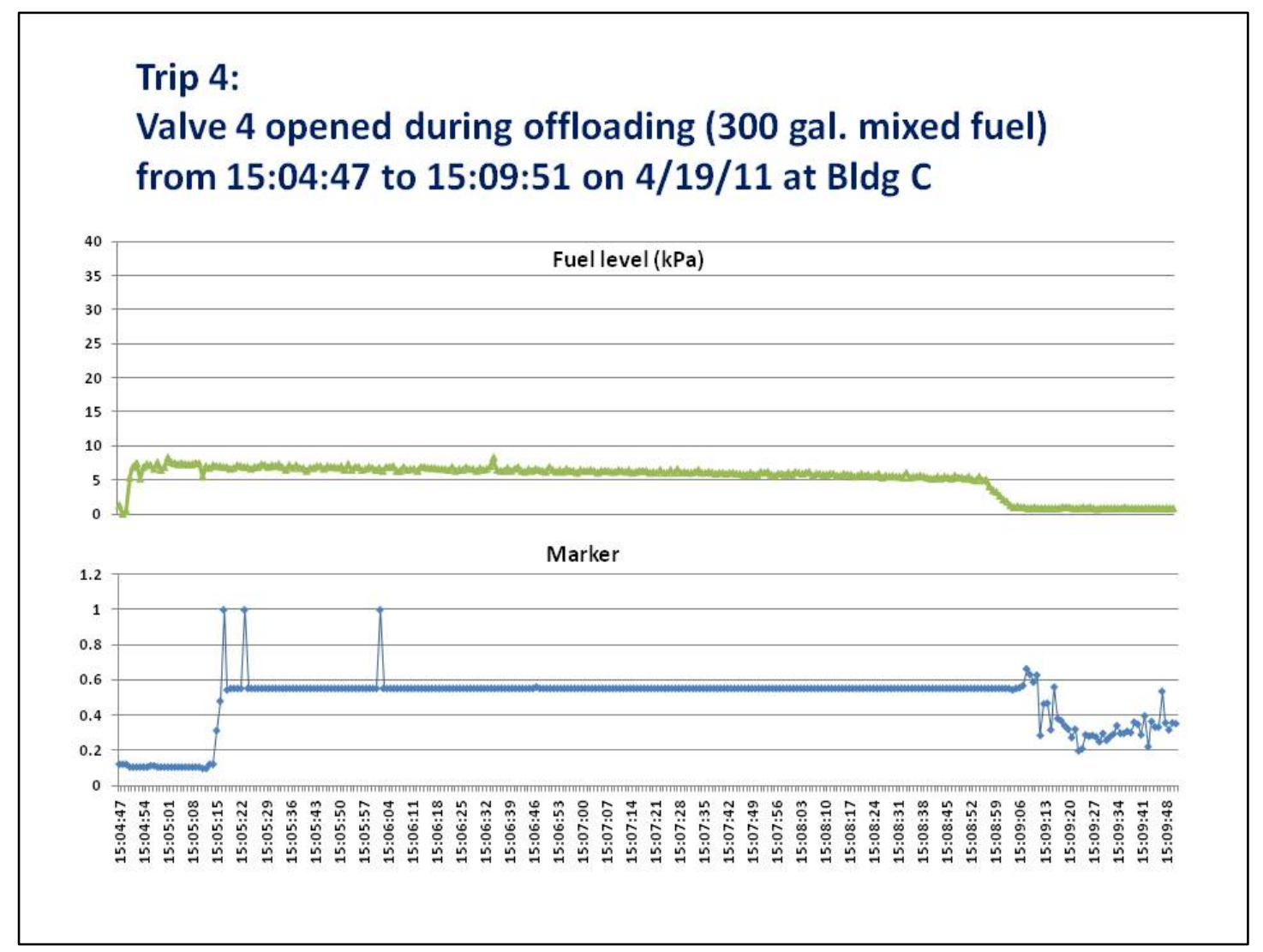

Fig. 105. Activity from offloading at delivery destination under Scenario 4.

\section{Warning level estimates}

\section{Unscheduled (second) fuel loading stop}

This was an unscheduled stop which would have raised some concern by itself already (without the benefit of sensor readings). The warning concern became more severe when the marker sensor detected the additional fuel being loaded into the tanker was basically unmarked (low value of 0.096). Thus the warning level estimated for this stop was $87 \%$; as such, the algorithm successfully detected a problem for this activity.

\section{Final destination stop}

At the final destination of this delivery, the marker concentration was detected at a significantly lower level than anticipated for marked fuel (0.354 instead of the original level of 0.936). This mixed fuel raised the concern and provided an estimated warning level at $63 \%$, which would raise an alarm for a reasonably suspicious activity.

\subsubsection{Blending Scenarios 2 and 3}

This was the most complex scenario tested at TRC, and simulated two additional types of blending. The vehicle's "assigned" route involved the delivery of two compartments of marked fuel (200 gal and 300 gal, respectively) at the fuel plaza after loading at Building C. Instead, compartment 1 of the tanker was loaded with the 200 gal marked fuel (as "assigned”), but then the vehicle made an unscheduled stop at the SE VDA where 20 gal of unmarked fuel (simulating hazardous waste or other substance) was added via the hatch. Furthermore, the 200 gal of fuel load into compartment 4 at the start of the trip were 
unmarked (rather than the 300 gal of marked fuel "assigned”), and at the unscheduled stop en route to the final destination, 100 gal of marked fuel were added. This simulated the addition of a lesser amount of marked fuel to unmarked fuel, making the entire load "look" marked (taxed). Both compartments were offloaded at the Fuel Plaza.

\section{Loading period}

Loading at origin (i.e., terminal)

The mounting solution for the valve sensor for this compartment led to data issues during this testing. The signal showed "valve open" continuously for a long period of time, during which the valve was known to actually be closed. This lasted several hours during some of the driving time and during the time when the tanker was idle while the team took their lunch breaks. Signal disruptions over this time period seemed to be more severe as well. This might have been impacted, at least to some degree, by the strong wind and heavy rain encountered the morning this scenario was tested.

The noise in the fuel level and marker concentration data (Fig. 106 and Fig. 107) were slightly higher than what had been observed thus far. Measurements for compartment 1 (Fig. 106) at the end of the loading were

- Marker concentration 0.582 and

- $\quad$ Fuel level 9.240.

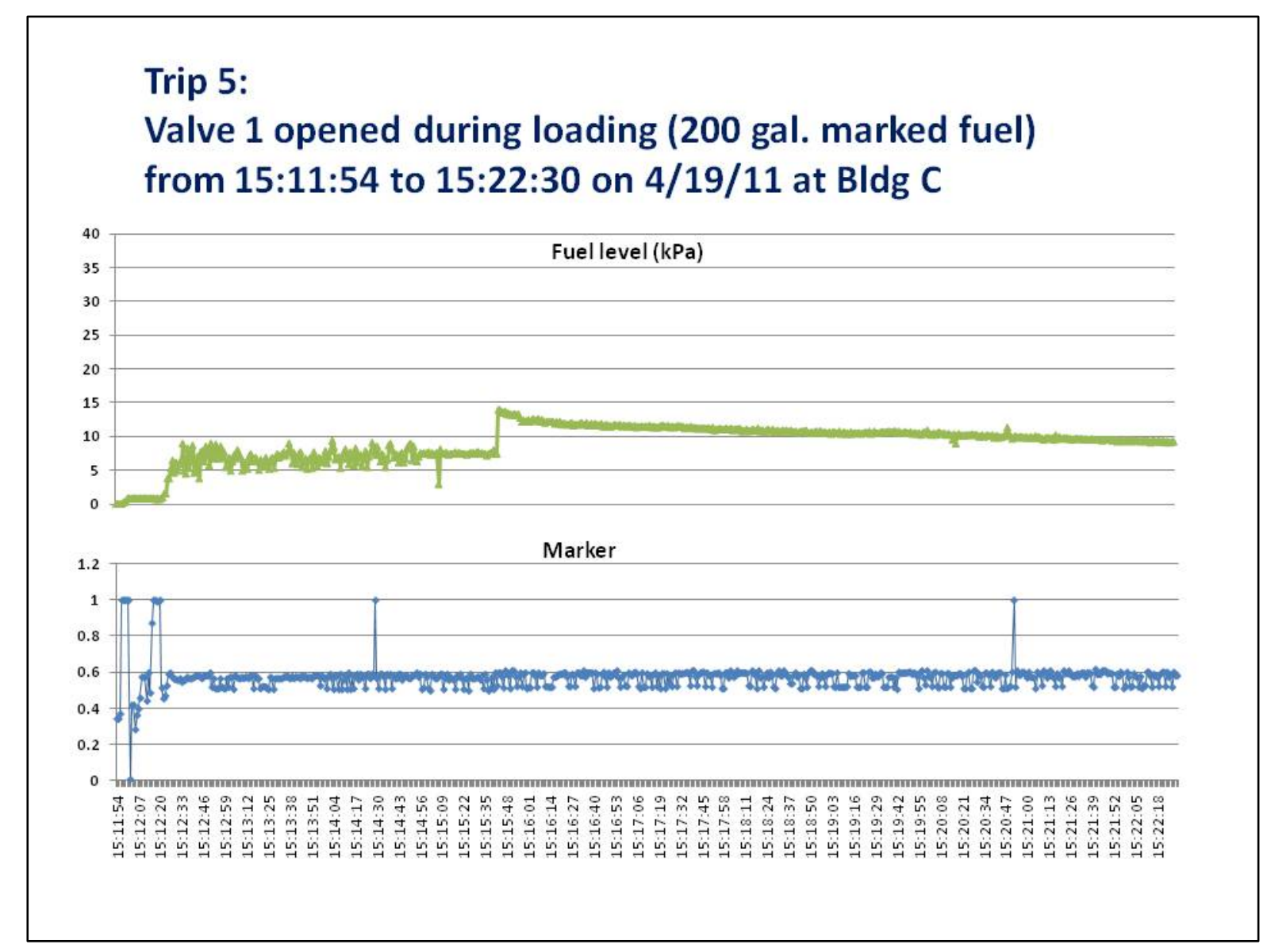

Fig. 106. Activity from loading of compartment 1 under Scenario 5. 


\section{Trip 5:}

\section{Valve 4 opened during loading (200 gal. unmarked fuel) from 15:22:31 to $15: 25: 47$ on 4/19/11 at Bldg C}

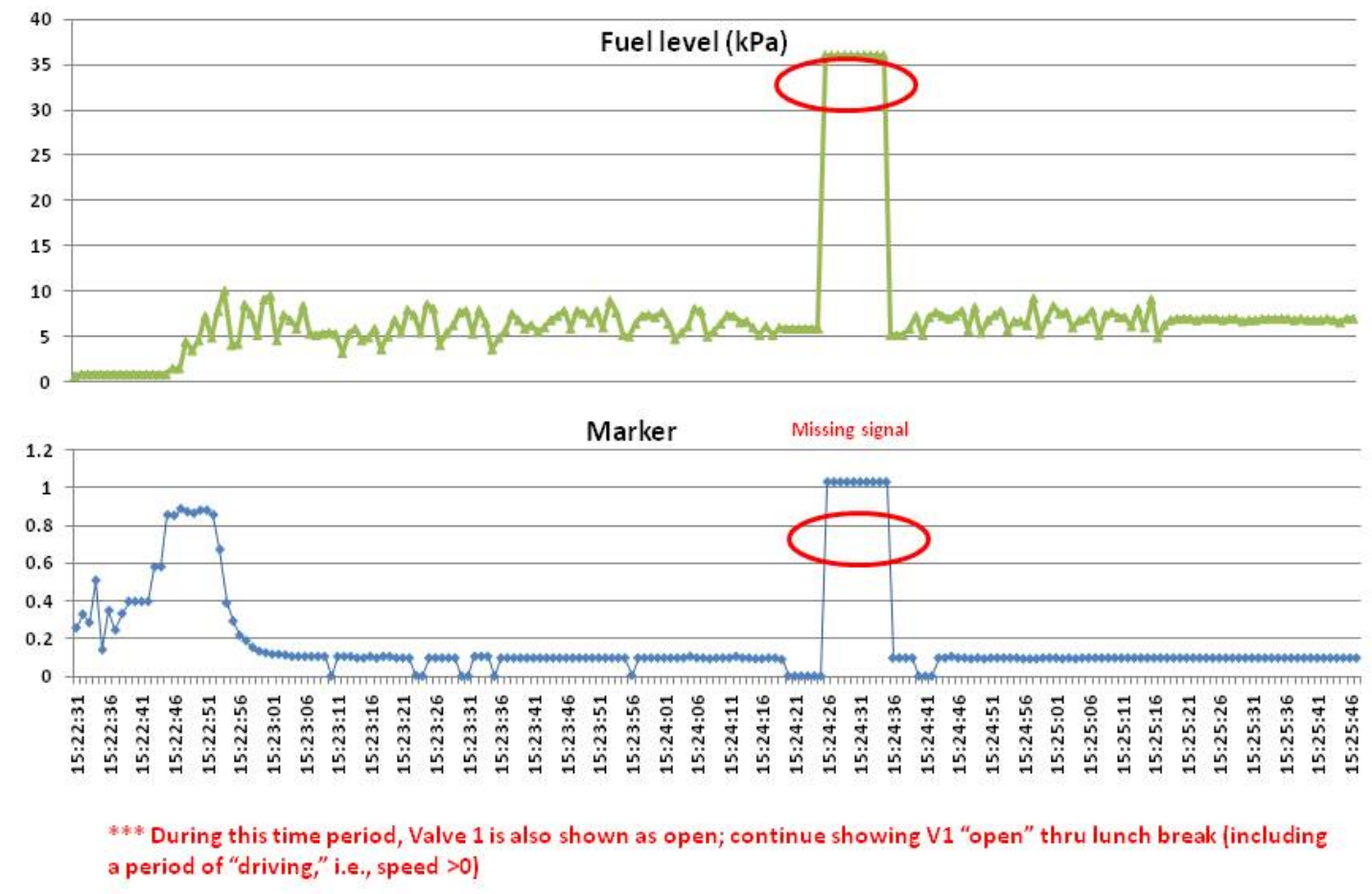

Fig. 107. Activity from loading of compartment 4 under Scenario 5.

Although unmarked fuel was loaded into compartment 4, the data from the optical sensor on compartment 4 still showed an appreciable (but low) level of marker concentration (indicating an error of around 10\% in measurement). Measurements from compartment 4 (Fig. 107) at the end of its loading were

- Marker concentration 0.096 and

- Fuel level 6.930.

\section{Unscheduled stop with hatch loading}

As a part of this trip, an unscheduled stop with 20 gal unmarked fuel added into compartment 1 of the tanker was performed. Because there was no other sensors (for fuel level and marker) present on the top of the tanker (only a hatch switch) no fuel pressure or marker concentration changes could be measured. Thus, the only available indicator that something unusual was occurring was the fact that the hatch was open and it was at an unscheduled stop. In this case, however, the hatch was open for only 44 seconds. With such a short period of stop, one could easily conclude that as a traffic stop (e.g., stop sign, or waiting for a turn). Therefore, under this scenario only the "hatch open" indicator was flagged in the data. 
Same unscheduled stop with additional fuel into compartment 4

At the same unscheduled stop location as above, an additional 100 gal of marked fuel were added into compartment 4. Higher noises in fuel marker and fuel pressure measurements can be observed in Fig. 108. Specifically, the measurements estimated at the end of this loading were

- Unscheduled stop,

- Marker concentration: 0.836, and

- $\quad$ Fuel level: 8.890.

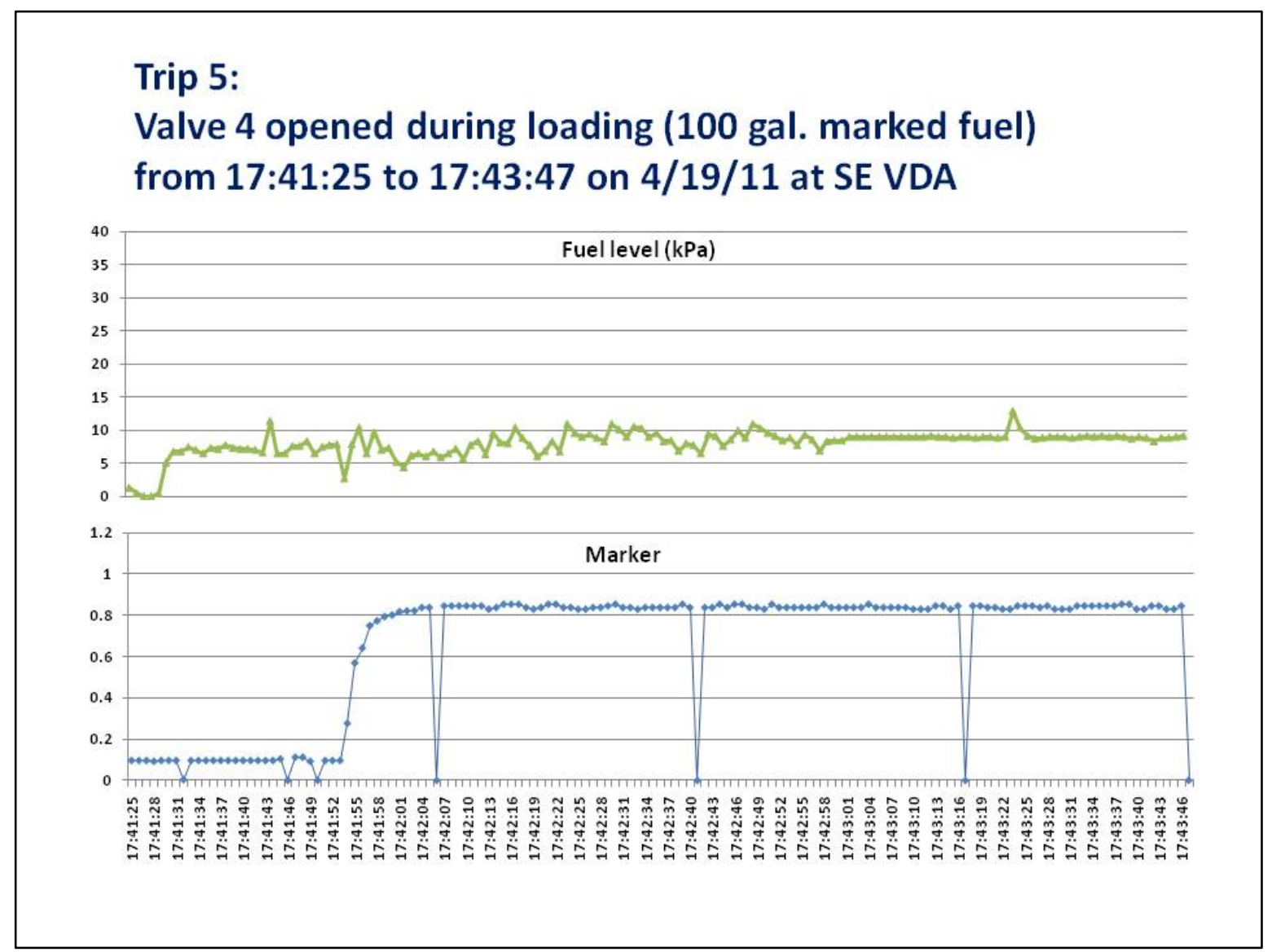

Fig. 108. Activity from loading at unscheduled stop under Scenario 5.

\section{Off-loading periods}

At the final destination of this scenario, more than the expected amount of fuel was offloaded from compartment 1 (220 gal instead of 200). Similar levels of noises in fuel pressures and marker concentration were seen in this data as observed in previous scenarios (Fig. 109). The fuel level did not drop completely to zero where it should be empty at the end of the offloading, however. The measurements showed

- Scheduled stop,

- Marker concentration: 0.680, and

- Fuel level: 0.980. 


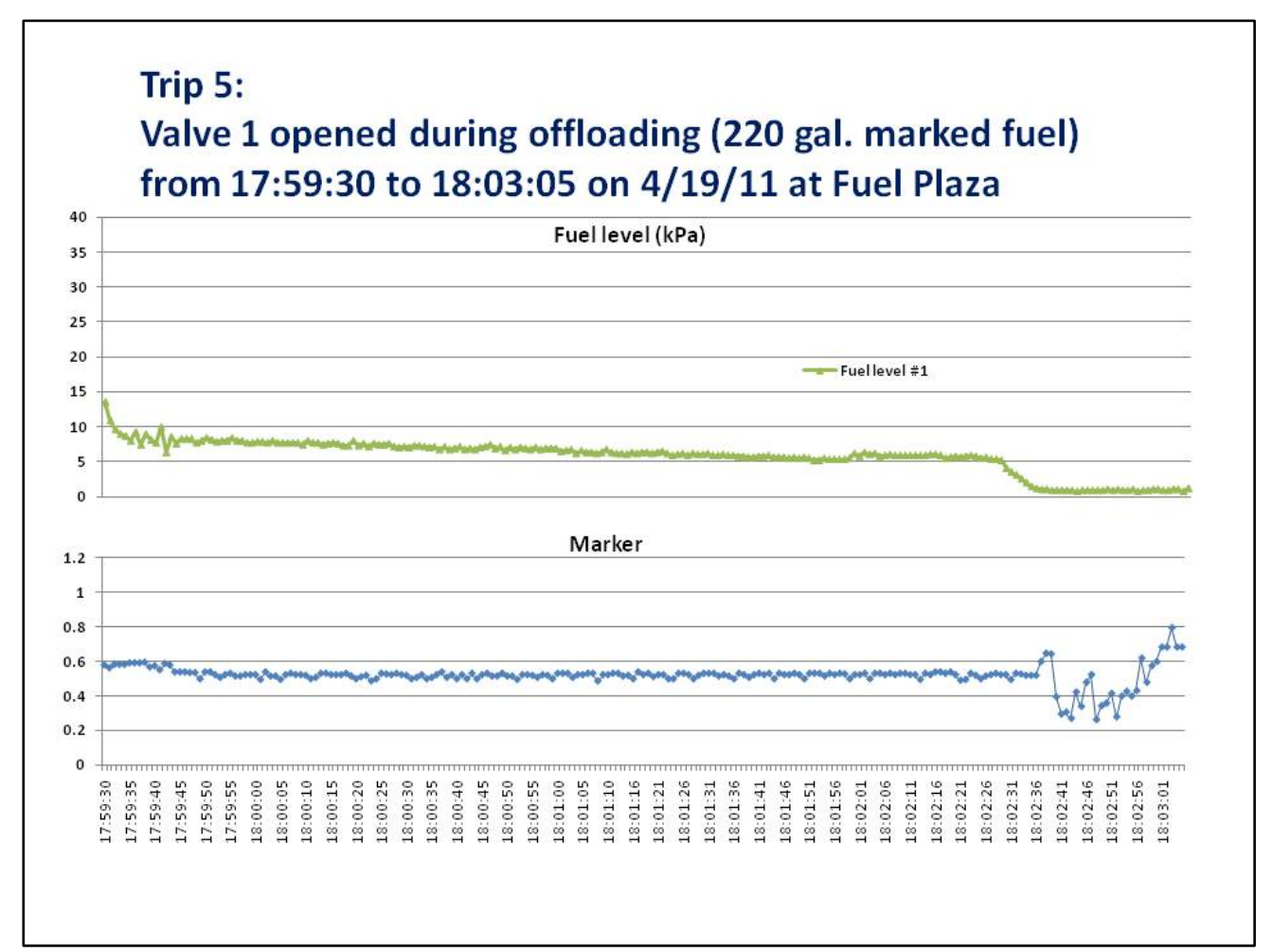

Fig. 109. Activity from offloading of compartment 1 under Scenario 5.

As in data collected from compartment 1 , similar levels of noises in fuel pressures and marker concentration were presented in the compartment 4 measurement data (Fig. 110). The fuel level also did not drop to zero where it should be empty at the end of the offloading. The measurements from compartment 4 are

- Scheduled stop,

- Marker concentration: 0.264, and

- Fuel level: 0.840

\section{Warning level estimates}

\section{Unscheduled loading stop}

At this stop, two parts of warnings could be detected. First, although no measurable quantities (see discussion above) could be used from the sensors, a hatch-open indicator for compartment 1 was detected to reflect a potential problem. Second, the fuel marker level for the additional fuel being loaded into compartment 4 was significantly higher, indicating marked fuel was flowing into compartment 4 (which should have been holding unmarked fuel per information obtained during the loading period). Thus, the warning level from this stop was estimated at $98 \%$. This provided a very high level of certainty regarding the warning.

\section{Final destination stop}

At the final destination of this delivery, the significant change in marker concentration from compartment 1 was detected. Adding to the unscheduled stop issue earlier, this removed any uncertainty in the concern and provided an estimated warning level of $100 \%$. Similar results were also observed in compartment 4 data, leading to a warning level for compartment 4 which was also at 100\%. 


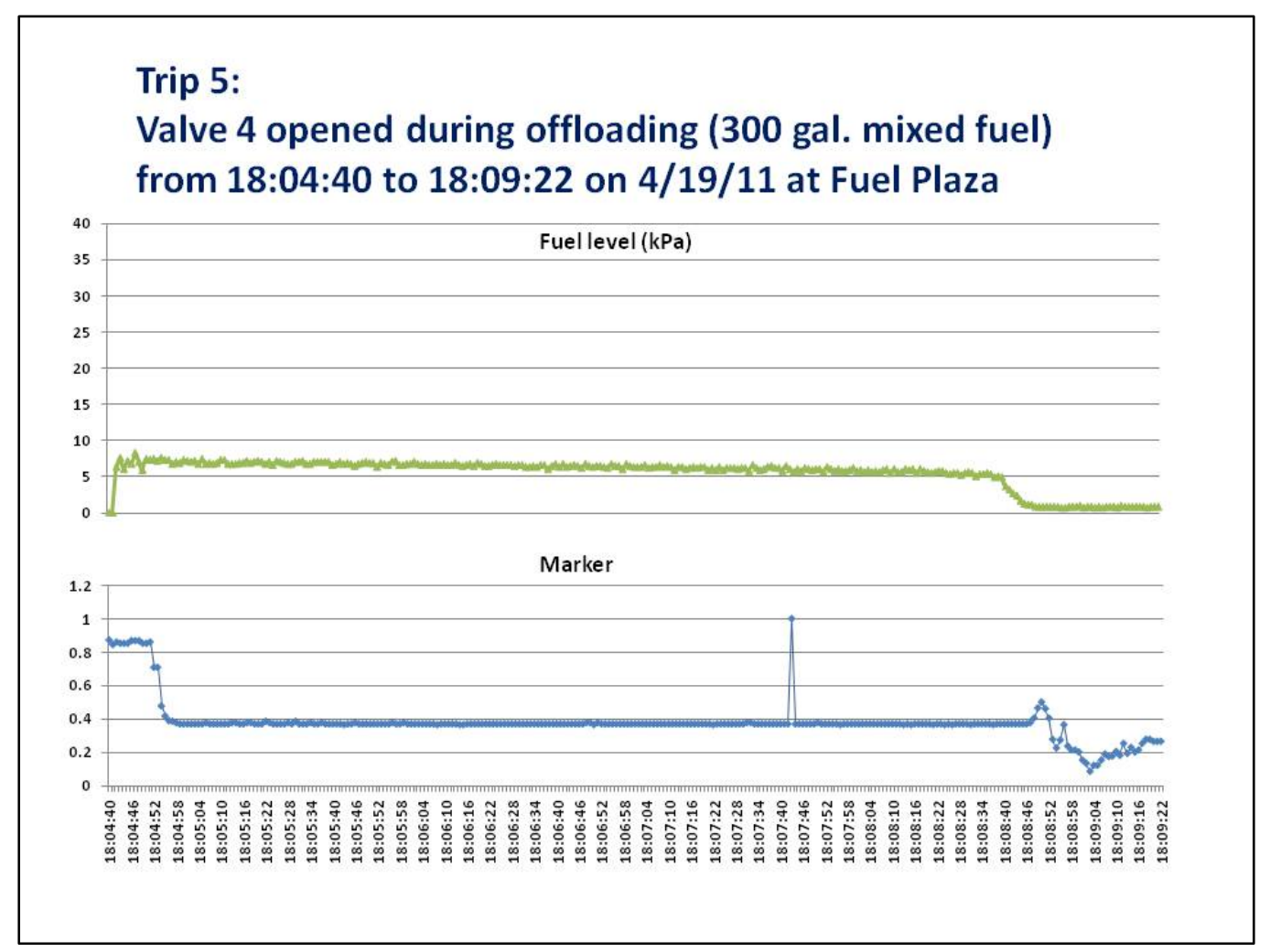

Fig. 110. Activity from offloading of compartment 4 under Scenario 5.

\subsubsection{De-Instrumentation}

The Pilot tractor-trailer was de-instrumented at the conclusion of the second day of testing with the exception of installing the original sight glasses. ORNL staff returned to TRC to oversee the installation of the original sight glasses on the Pilot trailer and to give instructions as to the reloading of the fuel borrowed from Pilot for the testing. The Pilot driver returned later that morning and received the test vehicle that had been restored to original condition. 


\section{LESSONS LEARNED}

\subsection{APPROACH AND METHODOLOGY}

The nature of the POC testing precluded the ability to collect data from a vehicle hauling fuel in a realworld environment due to the fact that the sensors and electronics had not been certified for use on tankers hauling flammable liquids and due to the need to very closely control the sequence of test events. We found that the decision to conduct the testing at a test track proved to be an acceptable alternative to real-world testing. The public was not exposed to the testing in any way, and all procedures, equipment, and installation methods were pre-approved by the Safety Committee at TRC. Further, the installation of the equipment, the driving of the vehicle, and the transfer of the fuel was all handled by TRC staff.

\subsection{FUEL MARKER}

Three primary lessons were learned in the development of a fuel marker chemistry for the FTE POC. First, it is imperative that the stability of the fluorescence signal of the marker be tested over weeks or months, not days. Second, the aging must be done within a diesel matrix at representative temperatures, not just a "pure" industrial solvent surrogate such as a toluene-isopropanol-benzene mix, as this lacks the heterogeneity and chemical complexity of real diesel. As we have seen, components of diesel such as naphthenic acids can react with and diminish or deactivate entirely the fluorescent signal from a potential marker. Third, the subtleties of organic dyes and organic-inorganic complexes cannot be overlooked, including the fact that, during the synthesis of a dye, multiple differently fluorescent by-products or incompletely reacted reagents or intermediates may remain. The optical signal from these parasitic yields will degrade in a far different way from that of the purified product. The process of purification becomes the main source of expense in the preparation of a marker chemical due to separation apparatus, wash solvent consumption, and labor hours to execute the purification and collect the purified product.

As field tests approached, significant progress was made to address the minor variations in fuel chemistry that had the potential to affect absolute marker concentration and thereby impact both the ultimate cost and sensitivity of the marker sensor measurement. Specifically, regional and seasonal variations in the density of diesel were carefully evaluated to assess how best to add marker to test fuel and, in a broader sense, how much accommodation for the normal variation in diesel density should be made as the monitoring method is standardized and a protocol written. Why, if the marker sensor reading is a relative measurement made within the frame of a single truckload of fuel, should such a seemingly negligible density variation be tracked? It must be accounted for, no matter how small, because bounds must be placed upon every variable that could effect change in the apparent marker concentration. In defining the greatest reasonable potential variation in fuel density, marker concentration variations detected by the sensor can more accurately reflect the possible range of unauthorized dilution. Density variation and its effect on concentration follows. In the most fundamental definition, density measures the amount of mass per unit of volume in a substance. If "more” molecules of fuel are packed into the same volume (i.e., higher density), slightly more marker molecules would be required to mix into that matrix to yield the same sensor signal. For this reason, it was imperative that the fuel marker team equip the sensor team with the density variation value so that marker sensor values could be assessed in consideration of seasonal and regional variations impacting physical properties of the diesel fuel. During the winter months, more volatile, lower molecular weight components are included in diesel blends to avert any of the "waxing out" of the fuel during exposure to temperatures below $20-40^{\circ} \mathrm{F}$. This waxing out or gelation, measured with the Cloud Point Test, is the process by which paraffinic fuel components aggregate and form insoluble particles that can render fuel transfers difficult and compromise engine efficiency. Similarly, during the summer months, diesel fuel blends are enriched with less volatile compounds to lower vapor pressure and increase total heat capacity of the fuel. 
It was found that, in general, the density values for diesel vary in greater degrees season to season within the same region than values vary region to region within the same season. However, broad variation in density does exist when comparing region-to-region, same season in climate extremes such as Minnesota versus Arizona. The absolute ranges of density values were difficult to pinpoint, in large part due to the proprietary nature of fuel blending information. Literature searches and discussions with ORNL's Fuels, Engines, and Emissions Research Center's senior fuel scientist Bruce Bunting (formerly of Cummins and Amoco) led to a final assessment of diesel density variation of $2.9 \%$ to encompass both seasonal and regional differences.

Finally, it was found that any residual concentrated dye deposited in dead volume or semi-dry metal surfaces within the tank, preventing the calculated concentration of dye from fully mixing with the total volume of diesel. As was seen in the Fuel Loop testing at the test track in East Liberty, Ohio, in April 2011, this type of residual undissolved marker creates a situation of constant "dosing" within the tanker and leads to an initially false-low marker content reading and an increasing upward drift in the marker concentration. A precise dosing mechanism for the marker into the fuel is needed. It is not known whether existing systems for dyeing agricultural diesel could provide adequate precision for dosing the highly concentrated SiNaph into diesel fuel.

\subsection{SENSORS}

The valve sensor proved somewhat challenging due to the fact that the valve handle had considerable freedom to move as the trailer vibrated in over-the-road operation. To mitigate this variability, the mount for the valve proximity sensor was designed to allow for adjustment in the field. Therefore, a position could be chosen that minimized the unwanted effects of vibration. However, even with this adjustment, early field testing data showed that when the vehicle was in motion the vibration produced some spurious and erroneous signals. To correct this issue during testing, the valve handle was taped in position during transport over the road.

During the field tests, the isolation valve for the pressure transducer was found to be mostly unneeded. The impulse pressure from the transfer pump used at TRC was found to produce only mild fluctuations in fluid pressure. These fluctuations were below the levels required to damage the pressure sensor. The bleed valve, however, was found to be useful in certain tests. Because the diesel trapped in the line between the trailer bottom valve and the "belly" valve experienced changes in pressure as the ambient temperature varied, it was often necessary to bleed the pressure to validate the pressure sensors operations. Likewise, the custom sight glass that served as a mount for most of the sensors would experience some level of leaking around the bolt holes due to pressure build up. In a real-world application, these components would likely "screen-out" as the liquid level measurement would be made from within the tank compartment, not in the fuel transfer plumbing.

A number of lessons learned were related to the instrumentation of the truck. First, there were Leak issues with the 7-bolt (versus 8-bolt) configuration on the sight glass flange. This issue could be avoided with an alternate optical sensor mounting solution to allow all eight flange bolts to be utilized.

The torquing of the bolts was an issue as well; these originally were not tightened enough by the TRC technician who did the installation because he was afraid of damaging the sight glass. This was corrected onsite prior to testing, and it was later determined that they torqued at about $20 \mathrm{ft}-\mathrm{lb}$. In the future, the proper torque specifications should be determined prior to testing for a proper mounting of test items.

Leaks were also a problem with the pressure sensor attachment; the threaded sleeve into sight glass was not properly sealed and tightened. A TRC technician was able to tighten without have to remove sight glass assembly from trailer. A short-term solution could involve mounting the pressure transducer directly 
to the sight glass flange, sealing with Teflon tape. Future prototypes will likely have a level sensor in tank compartment to allow level readings at times beyond when the belly valve is open.

Because of the location of the pressure transducer, the pressure reading was only valid when the belly valve was open and the valve for fuel loading/unloading was closed. Ideally, the level sensor should be in the fuel compartment to afford more accurate and longer duration readings. The sight glass location was selected for testing due to the requirement not to permanently modify the trailer or install sensors in the fuel compartment. This will not be an issue for real-world systems with an in-tank sensor.

Due to the location of the pressure transducer and restrictions from tubing/plumbing connections and trapped air, there was concern over the accuracy of the pressure transducer. It was found that when the fuel temperature changed, the pressure transducers went off scale (for temperature increases) or to zero (for temperature drops). This was due to the fuel expanding or contracting in the deadheaded section of piping. This issue would also be corrected by using an in-tank level sensor.

In addition to the marker sensor noise, the fuel level (pressure sensor) also had a higher-than-expected noise level. We also assumed that the fuel level will drop to zero when the tank is off loaded, but this appeared to be not always the case. This also points to the need for use of an alternate level sensor and alternate location (in the tanker compartment).

Apparently, the Air-Weigh self-weighing system was not calibrated when it was installed by the AirWeigh technician in Knoxville. This was not identified until the Air-Weigh tech supporting our upfit noticed it during the installation of the new ComLink to support the T2TCU signals. We did a calibration at TRC using their certified scales. We added what weight we could (1000 gal of fuel), but the calibration would have been more accurate across the full range of weights encountered in normal operation if we could have fully loaded the tanker. This and other integration issues would have been determined in a "test fit" of all components and communications paths prior to the trip to the test track. This was not possible due to the nature of the test vehicle's operation; however, in the future, every effort should be made to coordinate a time for such a test of instrumentation prior to extended testing. This should include a full "shakedown" of the system and exercising at least one of the test scenarios to validate full system functionality and to identify flaws in procedures, methods, and assumptions.

The marker sensor was a prototype and was not shielded for noise. Noise was an issue and caused problems for the Evidential Reasoning Team. Also, the sampling rate of the sensor communications system $(1 \mathrm{~Hz})$ was low relative to the noise, and thus it was hard to determine if a given output was a real reading of the fuel marker concentration or just an abnormal reading attributed to noise. A suggested improvement would involve shielding for RF and perhaps a faster sampling rate in conjunction with onboard filtering.

The suite of marker sensors (three total, including one spare) did not have the same reading for the same fuel marker concentrations. The output of the marker sensors was relative and not exact for the same fuel sample. Again, this caused problems for the FTE Upload Data Service software. For future work, a design and/or calibration should be explored that provides more standardization, giving the same output for the same marker concentration.

In general, the fuel marker sensor performed as expected. However, the noise levels associated with this sensor during the field testing was higher than those recorded during preparations for the test. Further investigation of the problem showed the radio frequency (RF) interference at the test track was to blame for spurious signals that showed up in the sensor's output. 
For the POC test, the sensor's housing was made of plastic because the plastic housing could be easily fabricated and modified. Because plastic is a poor barrier to RF radiation, it was possible for RF signal to enter the sensor's electronics package and produce unwanted signal fluctuations at the IC photodetectors and amplifiers. These signals resulted in noise as shown in Fig. 111.

In addition, the plastic housing was poorly suited for waterproofing the sensor's electronics. This was known in advance of the field test, but for cost and design reasons, the plastic housing was not replaced with a metal housing. Although this was a prototype system, we did experience heavy wind and rain during the field testing. Silicone caulk, tape, and plastic sheeting were used to protect the sensors enough that they functioned well during the 2 days of actual testing. Future designs should take bad weather into consideration (even for testing) and not wait until real-world deployment of such features.

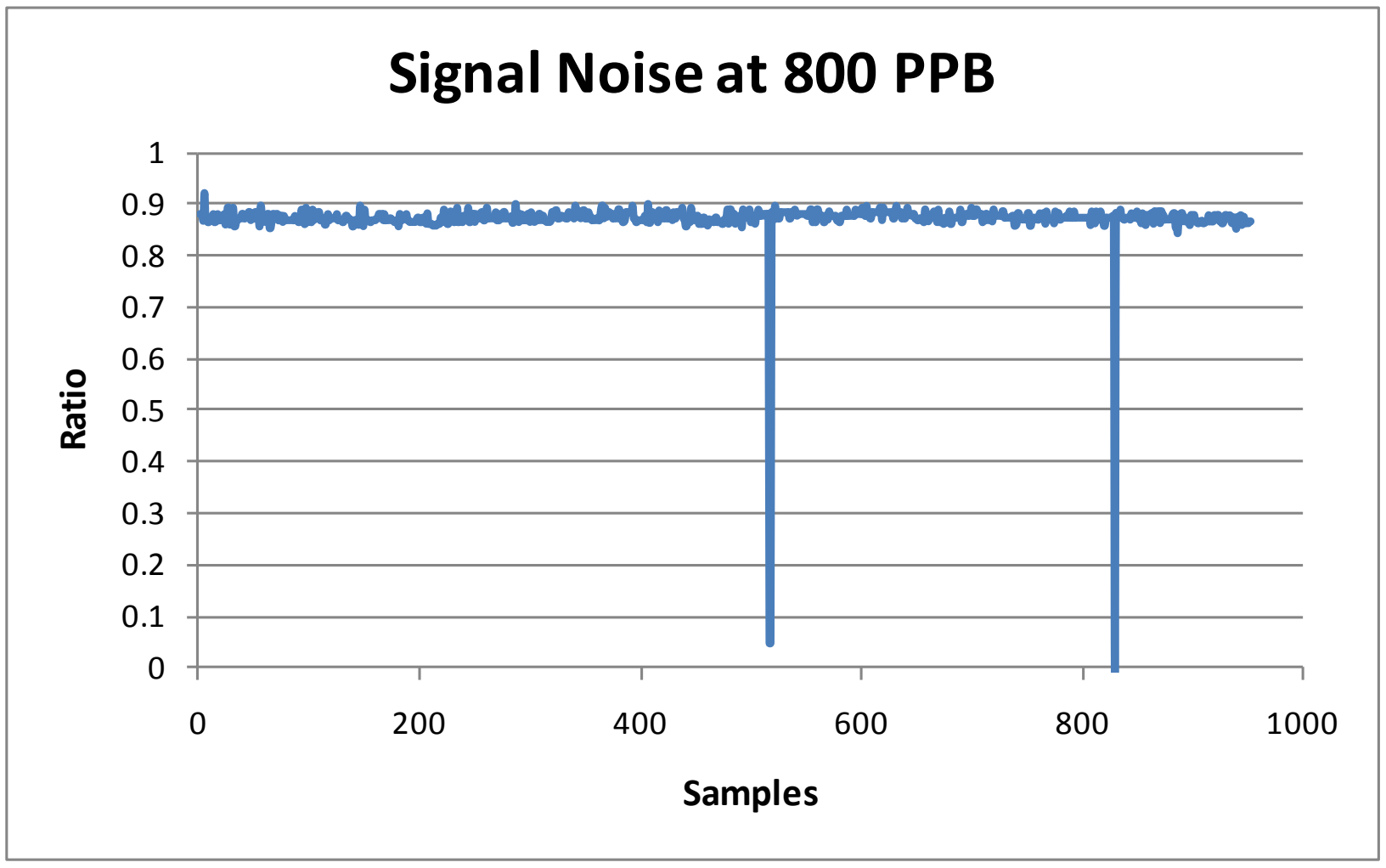

Fig. 111. RF-induced noise in prototype fuel marker sensor.

Because of the handle design of the fuel loading/unloading valve, it was able to move during truck movement, which caused the magnetic switches used on the valves to report open or momentary open conditions during movement. This could be corrected by screening out valve movement during times when vehicle movement is reported. However, depending on where the valve handle moves, it might show "open" during a stop even if it is closed. For future work, a new switch mounting solution should be explored, perhaps one where the switch is in the actual valve stem or other location that is not affected by the flopping of the handle. 


\subsection{COMMUNICATIONS}

The decision to use the ORNL DAS provided the needed flexibility for data retrieval in this POC stage of testing. During the preliminary testing at TRC, it was discovered that the initial data sampling rate of $0.1 \mathrm{~Hz}$ was too low. Because the data retrieval component was under ORNL's direct control, this could be altered with a relatively minor software change.

Another issue encountered during installation was that the truck battery went dead due to the drain encountered from the truck doors being open and equipment being powered up that was connected directly to the vehicle's batteries. This is a common problem during an instrumentation upfit; vehicles of this type (long operating duty cycles) typically have weak batteries. This caused us to lose some data at the beginning of the first test. When the driver attempted to start the tractor once we initialized our DAS, the voltage drop caused the DAS to shut down (and we were unaware of this for several minutes of the initial testing). For future upfits, shop personnel should be asked to charge the truck batteries during equipment installation; in addition, the DAS should be checked for data transfer at the start of each test.

An issue related to the T2TCU was the crashing of data over the ABS power line (J560 connector). The ABS power line is used by the vehicle for communications beyond the FTE data. There were apparent data conflicts that caused our Air-Weigh T2TCU system to slow down as each moment passed to the point that the data that posted data was several minutes old rather than real time. This caused confusion and delays in getting the system working and ready for testing (about a day and a half of delay overall). This problem was solved by adding a power line (isolated from the truck battery by using an AC inverter in conjunction with a DC power supply to convert back to DC) directly from the trailer unit to the cab unit. Such a cable would not be practical in the real world. However, Air-Weigh stated that they have a filter for such problems but did not send us one because they did not think it would be needed given the age of the test vehicle. This filter would need to be tested to be sure it corrects this problem for future systems. This problem was not initially identified in Knoxville because we never actually installed our equipment on this specific tractor.

The TRC campus has several areas of no cellular phone signal. This caused a loss of data to the back office. (A local copy of the data was on the DAS, so no data was actually lost. It was just not available in real time.) This points to the need for some on-board processing of data and decision making in addition to a communication component to resend key data later if a communication link cannot be established. Otherwise, loss of cell signal will render such a system useless during times of signal loss, which could be of long duration if they coincided with the vehicle being parked for fuel loading/unloading in a cellular dead zone. A number of factors prevented the discovery of this communications issue during the communications testing conducted at TRC several weeks prior. First, the vehicle did not always park in the exact locations used in the testing, although the general areas (WRC Pad, Building C, etc.) were the same. Second, the weather during the testing was much worse, hampering both cellular and GPS reception. Finally, the DAS was placed down inside the cab of the truck; during the earlier tests, the DAS was placed on the seat of the passenger car.

\subsection{EVIDENTIAL REASONING}

With the exception of the GPS data, all "sensors' responses" were simulated using the sensor simulation unit during the pretest. Thus, for the evidential reasoning team, the purpose of this pretest at TRC was mainly to be familiar with the site and its configurations, in terms of areas where the tanker would travel during the field test. An important purpose of the pretest was to test the coordination and data transfer between communications team systems and those of the evidential reasoning team using the DAS system. It was discovered after the pretest that certain short durations on the simulated activities had resulted in missing signal responses from the sensors (i.e., an activity started and finished within the 10 second data 
interval originally set for the pretest). Thus, the data collection rate was increased from $0.1 \mathrm{~Hz}$ to $1 \mathrm{~Hz}$ for the actual tanker testing, keeping the 5 min interval of transmitting data. This data collection and transmission rate was used during the field testing. Based on information gathered through the field test, this rate of data collection appeared to be appropriate. Under real-world conditions, however, this frequency rate may not be practical. Additional research involving real-world operations would be necessary to determine proper and reasonable data collection and transmission rates.

Based on data gathered from the fuel loop testing conducted during the pretest, the team realized that there were significant noises in the fuel marker measurements, particularly those measurements collected during the beginning of fueling or offloading process. The general consensus of the team was to use measurement readings obtained from the period immediately prior to the valve closure for the evidential reasoning algorithm. This method was implemented in the analysis of data collected from the scenario testing with the instrumented tanker. As shown in the field test results presented in Sect. 6, appreciable signal noise also could be seen in data collected during the time period immediately before the valve was closed. There seemed to be a correlation between the noise level of the marker concentration signal and the fuel level; the noise tended to be more significant in the marker measurement when the fuel level was low (typically at the end of off-loading fuel). This indicated that data obtained from the end period (i.e., period immediately prior to the valve closed) was not as stable as originally expected, and thus it might not provide accurate information for evaluating "evidence” (i.e., examining variations or changes in sensor readings due to a given event). Additional testing would be necessary to derive methods for making the necessary adjustments to the data so that measurements collected from the sensors could be better used as inputs to identify abnormalities using evidential reasoning algorithms.

In terms of evidential reasoning algorithms, the method employed for the POC evaluated information regarding the following: scheduled/unscheduled stop, valve opened/closed, hatch opened/closed, fuel level, and marker concentration. The use of weight information from the GPS system as an input should be further explored. Since the weight was obtained independently from the GPS system and other sensors, it is expected that this information could improve the evidential reasoning algorithm, particularly in the event that a sensor is not functioning properly.

Since the evidential reasoning relied heavily on the input data from sensors and GPS systems, it is important to point out that any improvements in the data quality will have a direct impact on the results generated from the evidential reasoning analysis. The more the noise in data can be reduced, the more accurate the resulting estimates. Data collected from the TRC field test, in fact, was the first "real" data set to be processed by the evidential reasoning algorithm developed for this project. Thus, without any prior calibration (not possible due to lack of access to the vehicle), some unfavorable results from the system (or algorithm) would be expected. A lesson learned from this process highlighted the need for realistic data to ensure the success of an algorithm development. As in any model development, data is needed to train the models so that they can behave properly for the intended purposes.

The time sequencing of the data collection is also important and will reduce unnecessary data cleaning effort during the data analysis. From the data analysis point of view, each test scenario should be conducted in a logical sequence without interruptions. For example, when conducting one of the test scenarios at the TRC, after the initial loading, the tanker was moved to an unspecified location for a period of break and then returned to the initial loading place to restart the original sequence in the scenario. This testing break caused a certain skew in the time sequencing of the tests and additional effort had to be spent to identify and remove the portion of "unneeded" data prior to the algorithm operation. These added "events" (driving to an unspecified location, stopped for a period of time, and returned back to the original place) also caused some confusion during the examination of the GPS data. 


\section{ARCHITECTURE AND COSTS}

\subsection{RECOMMENDED ARCHITECTURE}

The following architecture recommendations for the fuel delivery truck based FTE detection solution are made as a result of information gained in the POC. The architecture described in this section could facilitate both real-time notification and auditing uses of the data collected. Interdiction may be possible with this architecture depending on how the real-time notifications are handled and the availability of enforcement resources. In order to conserve bandwidth, this architecture takes an exception-based approach with much of the data processing taking place in the on-board system. This design is based on the assumption that the dispatch is able to provide up-to-the-minute delivery instructions in an electronic form which can be used by an on-board algorithm.

\subsubsection{System Components}

The recommended architecture for the FTE detection system is based on the information available from the following sensors installed in each fuel compartment of the trailer: fuel level, marker concentration, valve status, and hatch status. Communications between the tractor and trailer is handled by the trailer-totractor communications unit (T2TCU). The trailer-side T2TCU monitors sensors and reports measurements to the tractor-side T2TCU via power line communications (or frequency shift keying). Upon receipt of the messages from the trailer T2TCU, the tractor-side T2TCU posts the messages to the J1939 data bus in the tractor. The telematics device in the cab reads the signals from the data bus and sends and receives information to and from the telematics back office system and dispatch. The telematics back office system (BOS) sends dispatch information (amounts, offloading locations, taxed/untaxed) to on-board telematics devices. The telematics BOS also receives information from the on-board telematics device and maintains records including hours of service information and loading/offloading incidents. In case of apparent fuel theft, an alert is passed to the fleet dispatch and may also be passed to a command and control center. The command and control center provides notification to enforcement as appropriate in cases of apparent fuel theft. The key components of this system are shown in Fig. 112.

\subsubsection{Data and Communications}

The data flow for the recommended architecture minimizes the amount of data transferred to the command and control center and the processing which needs to be done there. As such, the majority of the data processing takes place on board the vehicle while also balancing the complexity of the T2TCU and electronic on-board recorder (EOBR) processing algorithms.

Each compartment of the tanker trailer is instrumented with the following RS485 sensors (where signals are not directly available from the tanker electronics). The RS485 communication was selected because of the flexibility to handle a variable number of inputs (depending on the number of compartments):

- Hatch status (open, closed),

- Valve status (open or closed),

- Marker concentration (in ppb or similar measurement), and

- Compartment fuel level (calibrated to provide level estimate in gallons).

The trailer-side T2TCU monitors all sensors in all compartments at a rate of $0.5 \mathrm{~Hz}$ (every 2 seconds). The recommended architecture uses one-way communication from trailer to tractor to limit complexity and communication requirements. For normal circumstances when neither loading nor offloading is taking place, the trailer side will report that sensors are functioning properly at a rate of $0.1 \mathrm{~Hz}$ (every 


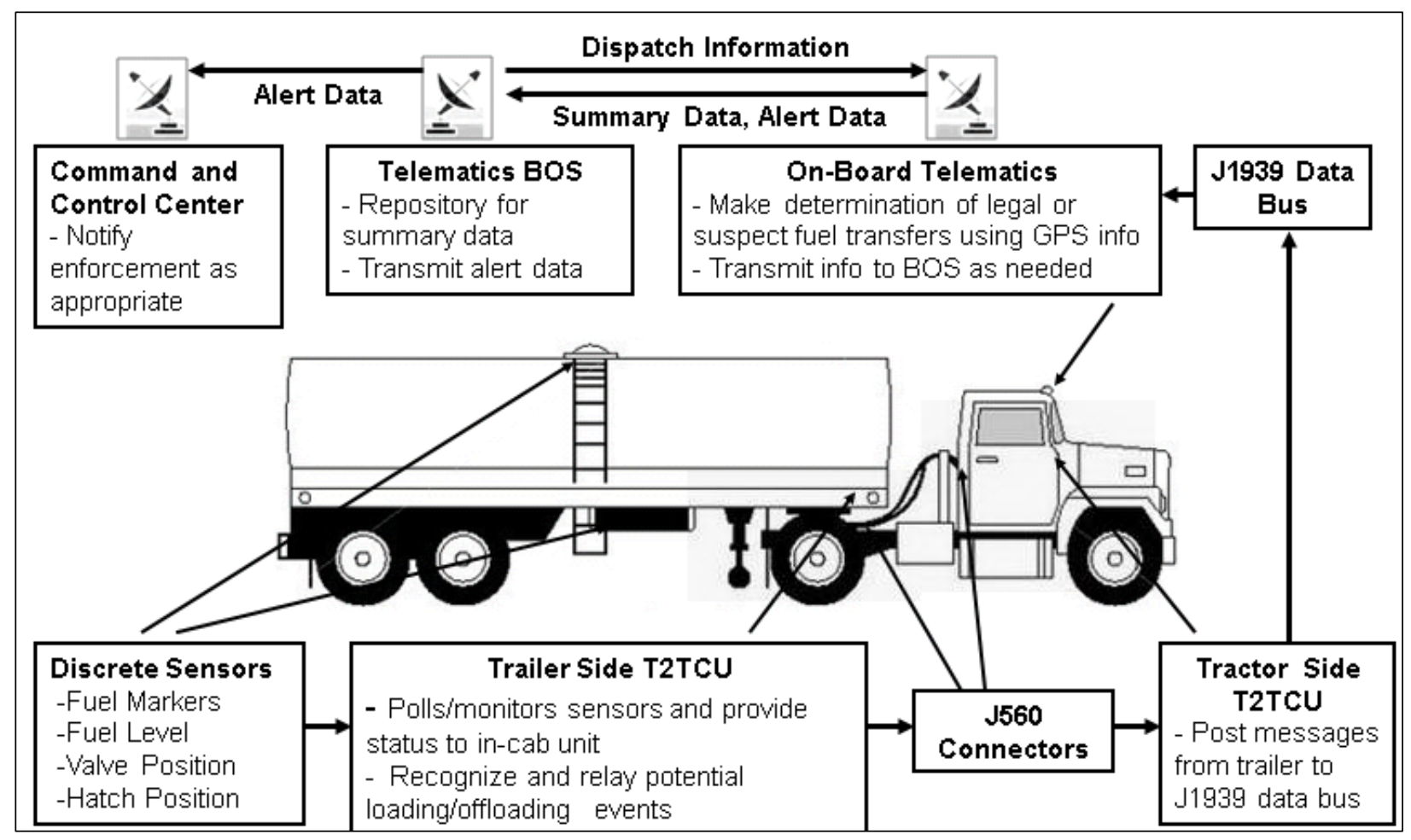

Fig. 112. Data flow for recommended architecture.

10 seconds). Otherwise, the T2TCU will report compartment-specific information (compartment number, level, marker reading, hatch and valve status) when any of the following conditions are met (and continue to provide updates to trailer unit at a rate of $0.2 \mathrm{~Hz}$ as long as these conditions persist):

- $\quad$ any trailer sensor goes offline,

- hatch is open,

- valve is open,

- marker concentration reading changes more than a certain amount (more than the tolerance of the sensor), and

- level reading changes more than a certain amount (more than the tolerance of the sensor). Note that this condition will typically be redundant with hatch or valve opening.

The tractor-side T2TCU posts messages from the trailer-side T2TCU to the vehicle's J1939 databus upon receipt. As described previously, a generic "sensors okay" message will be sent at $0.1 \mathrm{~Hz}$ (standard message indicating fuel transfer is not taking place and sensors functioning) or a warning message at $0.5 \mathrm{~Hz}$ (compartment-specific messages when conditions warrant additional data transmission as described previously).

The on-board telematics device contains a GPS unit, enabling it to process the received messages from the trailer (read from the J1939 data bus) in light of the dispatch messages to determine whether a potentially suspicious message is part of the normal, legal operations or not. The telematics device also functions as an electronic on-board recorder and may read other signals from the data bus for fleet management purposes. An application on the telematics device processes the data from the trailer and makes a determination regarding whether tampering, FTE, or regular legal operations are taking place. When fuel transfers are taking place, the on-board GPS information is compared to electronically received dispatch information from the telematics BOS. If the algorithm determines the activities are 
legal, a summary fuel transfer conditions (amount, type of fuel, compartment, and drop location) are stored on-board until the next regular upload period when the data is transferred to the BOS.

The algorithm will prompt the telematics device to provide immediate notification when the trailer goes offline during the course of regular delivery operations or fuel is being moved in amounts or at locations different from those received from dispatch. This notification will be sent from the telematics back office to the fleet dispatch and command and control center (from which it may be sent to enforcement as well if the situation warrants). Also needed is a way to handle interrupted communications (e.g., areas of poor cell coverage). The suggested algorithm makes use of a non-GIS approach, in which GPS data is only used to determine loading and drop-off locations, not to make routing determinations. Should a GIS approach be desired, it could be incorporated into the on-board functionality of the telematics device.

\subsection{COST ASSESSMENT}

The costs related to the implementation of the proposed architecture can be divided into five major groups: (1) sensor costs (including equipment and installation); (2) communications costs (from trailer to tractor and from/to vehicle); (3) telematics service provider cost; (4) cost of fuel marker (including chemical components and injection into fuel); and (5) cost of software (including development and deployment of evidentiary reasoning algorithms and GIS databases). Some of these costs are incurred only once during the lifetime of the equipment (e.g., sensor costs, software development cost), while other costs are recurrent (e.g., fuel marker, from/to vehicle communication costs, telematics service provider costs).

Table 6 presents a summary of these costs for the proposed architecture. In that table, the "from/to vehicle" communications costs and the telematics service provider costs are grouped into one item. This is because the proposed architecture only requires a minimal communication frequency with the command and control center, and in general this low frequency of communication is included in the telematics provider monthly service fee. For higher communication frequencies (discussed later in this section for an architecture that relies on a centralized system), the telematics service provider costs and the communication costs are separated.

In Table 6 the costs are not homogeneous (i.e., some are on a per vehicle basis, and others on a per month basis, etc.) and therefore cannot be added to determine the financial impact that the proposed FTE architecture could impose on fuel-hailing companies and ultimately on fuel consumers. However, assumptions can be made to compute a total cost per gallon of fuel that a FTE system would impose. These assumptions are as follows.

1. The life cycle of the sensors is 5 years.

2. Each vehicle makes four trips per day with a full tank (8,800 gal of fuel).

3. The development cost of the Evidentiary Reasoning Software is $\$ 1$ million and will be used by 50,000 vehicles.

4. The maintenance cost of the Evidentiary Reasoning Software is negligible.

With these assumptions, the additional cost per gallon of fuel would be $\$ 0.0702$. That is, the impact of the sensor/communications/software technology would be only $\$ 0.0002$ per gallon of fuel hauled (i.e., \$0.0702-\$0.07), with the bulk of the cost attributed to the fuel marker itself (note: even in an extreme case in which a tanker trailer only hauls a full tank once per week, the cost per gallon of fuel would be $\$ 0.0758)$. The total annual cost for the fuel transportation company would be $\$ 2,651$. 
Table 6. Summary of FTE-related costs for the proposed architecture

\begin{tabular}{|c|c|c|c|}
\hline & \# of units & Cost per unit & Total cost \\
\hline \multicolumn{4}{|l|}{ Discrete sensors } \\
\hline Hatch position sensors & 4 & $\$ 50.00$ & $\$ 200.00$ \\
\hline Fuel level sensors & 4 & $\$ 1,500.00$ & $\$ 6,000.00$ \\
\hline \multicolumn{4}{|l|}{ Vehicle weight sensors $^{a}$} \\
\hline Tanker trailer & 1 & $\$ 125.00$ & $\$ 125.00$ \\
\hline Tractor & 1 & $\$ 950.00$ & $\$ 950.00$ \\
\hline Valve position sensors & 4 & $\$ 50.00$ & $\$ 200.00$ \\
\hline Fuel marker sensors & 4 & $\$ 100.00$ & $\$ 400.00$ \\
\hline Spatial location sensor (GPS) & 1 & $\$ 0.00^{b}$ & $\$ 0.00$ \\
\hline Sensor installation & 1 & $\$ 800.00$ & $\$ 800.00$ \\
\hline Total cost discrete sensors (\$/veh) & & & $\$ 8,675.00$ \\
\hline \multicolumn{4}{|l|}{ Communications } \\
\hline T2TCU w/sensors 1st compartment & 1 & $\$ 875.00$ & $\$ 875.00$ \\
\hline Additional compartments & 3 & $\$ 225.00$ & $\$ 675.00$ \\
\hline Total cost communications (\$/veh) & & & $\$ 1,550.00$ \\
\hline \multicolumn{4}{|l|}{ Telematics service provider } \\
\hline Includes from/to vehicle communications & 1 & $\$ 50.00$ & $\$ 50.00$ \\
\hline $\begin{array}{r}\text { Total cost telematics service } \\
\text { provider (\$/month) }\end{array}$ & & & $\$ 50.00$ \\
\hline \multicolumn{4}{|l|}{ Fuel marker } \\
\hline Fuel marker cost & 1 & $\$ 0.07^{c}$ & $\$ 0.07$ \\
\hline Total cost fuel marker (\$/gal) & & & $\$ 0.07$ \\
\hline \multicolumn{4}{|l|}{ Software } \\
\hline \multicolumn{4}{|l|}{ Evidentiary reasoning application } \\
\hline Development $^{d}$ & 1 & $\$ 20.00$ & $\$ 20.00$ \\
\hline Installation & 1 & $\$ 30.00$ & $\$ 30.00$ \\
\hline Total cost software (\$/veh) & & & $\$ 50.00$ \\
\hline
\end{tabular}

\footnotetext{
${ }^{a}$ Assumes that the T2TCU is installed.

${ }^{b}$ Included in Telematics Provider's monthly fee.

${ }^{c}$ The cost of the fuel marker in mass production is as of 2011 about $\$ 0.72$ /gal; however, there is a significant probability that this cost could be reduced by $90 \%$.

${ }^{d}$ Assumes 50,000 tanker trailers.
}

\subsubsection{Real-time Interdiction System}

As described elsewhere in this report, a real-time interdiction system would require that as soon as a suspicious action by a driver is detected, the dispatcher be alerted. Early detection, in turn, requires the determination of a likely route that the vehicle would follow to its assigned fuel distribution destination; any deviation from that rout would set up an alarm. The Evidentiary Reasoning Software is capable of generating those routes and of determining when diversions occur. However, to be able to accomplish this, the software requires additional information, such as an up-to-date GIS database of the transportation network. 
The proposed architecture does not consider this capability. However, it could be added at an additional cost of about \$150/vehicle/year, which covers the annual costs of a GIS database license and some additional on-board hardware. Notice that all the other costs remain the same as indicated in Table 1, since it is expected that the need to communicate with the dispatcher would only increase slightly or (in most cases) not at all. In this case, and under the assumptions discussed above, the additional cost per gallon of fuel hauled would be $\$ 0.0702$ gal (i.e., same as before). In this case, the total annual cost for the fuel transportation company would be $\$ 2,681$.

\subsubsection{Centralized System}

The architecture proposed here is such that all of the evidentiary reasoning to identify a FTE event is performed on-board of the vehicle and only alarms are transmitted to the dispatcher or other interested parties. If a centralized system that is a depository of all the information collected and that houses the Evidentiary Reasoning Software is required, then the frequency with which the vehicle communicates with the central system would increase significantly. This in turn would increase the telematics providers fee to $\$ 100 /$ month or more, depending on how often the vehicle is required to submit the status of the onboard sensors (including spatial location). Under the assumptions discussed above, a centralized system would increase the cost per gallon of fuel hauled by $\$ 0.0703 /$ gal. This figure does not include the operation costs of the centralized system. For the fuel transportation company, the total annual cost would be $\$ 3,251$.

\subsubsection{Fuel-marker Break-even Cost}

The U.S. Energy Information Administration Annual Energy Review 2010 (Table 5.13c) ${ }^{9}$ indicates that in 2009 the daily consumption of diesel fuel and jet fuel in the United States was 2,600,000 and 1,407,000 barrels, respectively. With this information and assuming a tax evasion of $\$ 1$ billion per year, it is possible to estimate the break-even cost of the fuel marker (i.e., fuel-marker cost at which the dollar amount that would be recovered from avoiding tax evasion would be completely spent in adding the fuel marker to the fuel). Considering 42 gal/barrel, in 2009 the United States consumed 61,473M gal of fuel, which gives a break-even cost for the fuel marker of $\$ 0.016 /$ gal ( $=\$ 1 \mathrm{~B} / 61,473 \mathrm{M}$ gal). Therefore, to make economic sense, the fuel marker would have to cost much less than \$0.016/gal for an annual fuel tax evasion of $\$ 1$ billion. Conversely, and considering the price of fuel marker included in Table 6 (i.e., $\$ 0.07 /$ gal), the annual fuel tax evasion figure should be larger than $\$ 4.30$ billion.

This shows the very large financial impact that the fuel marker has in implementing this technology to avoid FTE. A system like the one proposed here (see Proposed Architecture above), but without the fuel marker component, would still derive most of the benefits at a very minimal cost. Such a system will still have the auditing capabilities; that is, all the fuel loading and unloading events would be registered with location and time, as well as all the hatch opening events. Therefore, if a hatch or valve vents occurs at an "unofficial" site, it could be further investigated. This system would also greatly reduce the costs for the fuel transportation company. For example, it will go from $\$ 2,651 /$ year to $\$ 720 /$ year (= $\$ 600 /$ year for the telematics service fee plus $\$ 120 /$ year for the cost and installation of the valve and hatch sensors amortized over a 5 year period), since only valve and hatch sensors would be needed (i.e., fuel level and vehicle weight sensors would not be a requisite for the system). Additionally, there are variants of this reduced technology scheme (e.g, hatch, valve and vehicle weight; hatch, valve and fuel level). 



\section{FUTURE DIRECTIONS}

\subsection{RECOMMENDATIONS FOR PHASE II}

\subsubsection{Approach and Methodology}

Real-world testing is needed to fully exercise the evidential reasoning algorithm and to validate the FTE concept. Clearly future test efforts must include real-world testing. However, in future phases of testing, the lessons learned from the POC cannot be ignored. Any future system will need to be qualified on the test vehicle(s) and at a test track before being placed in real-world testing.

\subsubsection{Fuel Marker}

Any further work in this area will benefit significantly in both a regulatory and environmental sense from an engine testing campaign of marked diesel fuel emissions and performance in the diesel engine test cells of the Fuels, Engines, and Emissions Research Center at ORNL. This would serve as a necessary validation of literature and mathematical calculation-based affirmations that the addition of SiNaph to diesel fuel does not compromise engine performance or create problematic emissions or deposits.

Going forward, the greatest challenge and opportunity associated with the fuel marker portion of the project is cost of the marker itself. Several approaches can reduce the cost of a chemical, and the most obvious, bulk pricing, was utilized to a certain extent in the POC phase of this work. By negotiating with the vendor (Sigma Aldrich, St. Louis, Missouri), the fuel marker team was able to reduce the cost of the SiNaph procured for testing by fivefold. However, deeper savings would be realized with the development of a streamlined synthesis and purification strategy that removes large commercial interests from the equation. SiNaph was pioneered as a complex molecule for photodynamic therapy in humans, so the development costs and licensing fee to Sigma were commensurate with a pharmaceutical, despite its being sold as a research chemical. The greatest potential area for cost savings in the synthetic route to SiNaph area likely lies in the man-hours required to properly purify the synthetic product, removing unattached cyanine compounds and silicon centers that are not fully complexed. A specialty synthesis route could be developed and handed over to a smaller chemical company more suited to the particularity of supplying low (compared to the bulk Sigma deals) and mid-range advanced synthetic chemical processing. This effort would be a collaboration between ORNL that would later be taken up by an outside entity.

To lay the groundwork for this prospective specialty chemistry research, contact was made and preliminary costing and feasibility questions were asked of a specialty chemicals company (Fig. 113) about establishing a specific SiNaph fuel marker dye bulk synthesis and purification operation. This route would only be cost-saving and applicable to a marking campaign of hundreds of thousands of gallons of diesel were to be marked.

A final cost analysis would not be possible until the sensor system's linear dynamic range had been decided upon and a more realistic estimate of cost added per gallon of fuel by the various markers (SiNaph and sister compounds, whether commercially available or newly synthesized) could be made based on more advanced instruments' actual calibrations and limit of quantitation data. In the meantime, some examination was made of potential extensions of the use of the SiNaph dye in more covert scenarios that still may relate to FTE, either with or without on-vehicle, real-time monitoring. The passive observation of SiNaph with light farther into the infrared region would exploit the longer wavelength excitation of the marker in order to achieve minimal interference by fuel handlers. Minimal handler 


\section{NovaChem: CONTRACT MANUFACTURING SPECIALTY CHEMICAL MANUFACTURING}

Nova offers chemical organic synthetic manufacturing services on a toll or contract basis. Our core expertise is in handling large volumes of flammable and corrosive liquids and is reflected in our facilities and experience. We have significant expertise in controlling exothermic processes and in conducting reactions at sub-zero temperatures.

\section{Reaction Vessels}

Glass-lined and or stainless-jacketed stirred reactors, capacities from 500 gal to 4000 gal. Reactors can be heated up to $400^{\circ} \mathrm{C}$ or down to $-15^{\circ} \mathrm{C}$. Our flexible and multipurposed equipment is designed for rapid deployment.

\section{Solvent Distillation}

Distillation columns available are both packed or tray configuration. Can be operated in full or partial reflux mode.

\section{Blending Service}

Nova offers custom liquid blending and formulation services.

\section{Drumming Service}

We offer a bulk solvent drumming and repackaging service. Bulk liquid can be delivered by rail or road tankers to our facility for storage in our bulk tanks or packaging into drum or smaller quantities.

\section{Warehousing}

Approximately 50,000 sq ft covered warehouse space is available for storage of flammable or hazardous materials.

\section{Environmental Compliance}

Our facility's air emission system is designed to contain VOCs and acids. Our wastewater system is governed by a NPDES permit for specialty organic chemical manufacturing. In most instances contract work can be undertaken without major permit modification.

Fig. 113. NovaChem capabilities for bulk manufacturing.

interference could facilitate maximized intelligence collection or enforcement because the subjects would be unaware their activities were being monitored. For instance, such minimally detectable observation approaches would lead to roadside sampling and/or inspection for tax status monitoring that connects a fuel sample to its sourcing locations by use of a specific dye undetectable with the unaided eye introduced in a meaningful fueling location. This utilization of the fuel marker could serve as a secondary check of either dilution or illegal sourcing after an interdiction has been made, although the sensitivity of the lawenforcement-borne visual detection system would fall well below what the on-board sensor could detect.

\subsubsection{Sensors}

Several improvements to the hatch, valve, and level sensors could be envisioned in a Phase II effort. For the valve sensor, one of the following options may prove viable. First, it may prove viable to use the valve mounting holes to design a sensor that detects the removal of a valve dust cover as opposed to handle actuation (Fig. 114). 


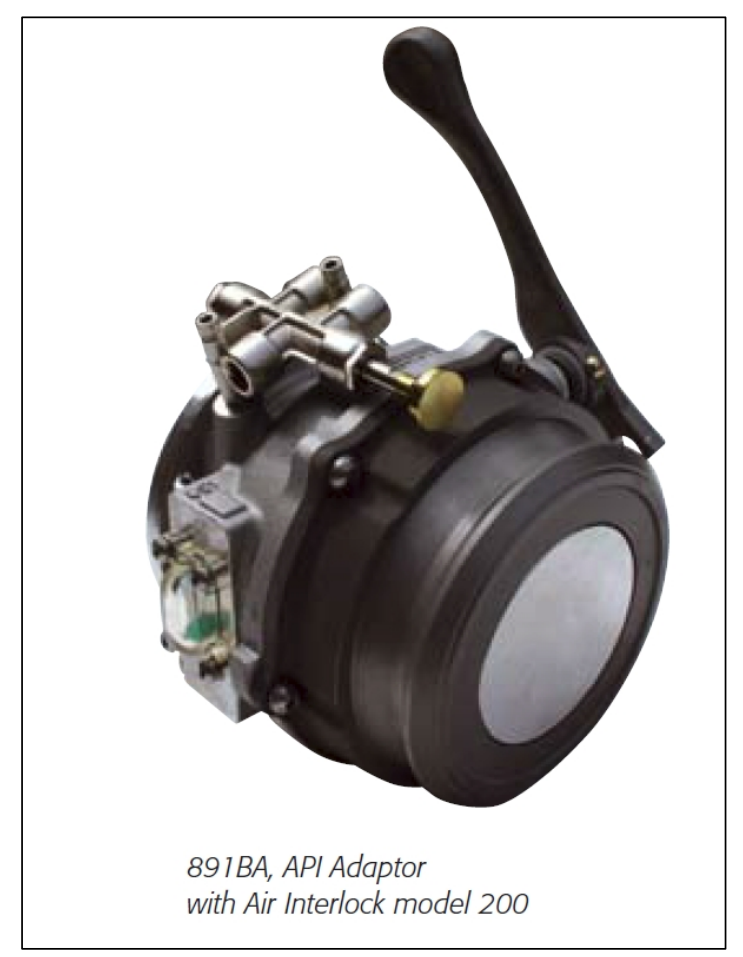

Fig. 114. Valve with dust cover sensing apparatus.

Another option might be to replace the valve handle sensor with an emergency valve opening sensor. Such a sensor would tie into the electronics controlling the emergency valve. Alternatively, it may prove acceptable to instrument the "safety bar" across all four valves in Fig. 46 so all valves are monitored with one sensor. In the next phase, options should be explored for COTS sensors that are less expensive than those used in the POC while meeting safety requirements.

Similarly, alternatives for hatch sensing should also be explored. One possibility is to put in a "bar" across all four hatches that will only require one sensor to detect motion. This would also reduce the potential of removing an entire hatch to defeat the sensor; the hatch assembly itself is attached like a drum lid and can be removed in whole. Another possibility would be to use a photocell inside compartment instead of a proximity switch to determine if the compartment hatch is open.

While several alternative technologies which may prove feasible for level sensing may exist, some modification will likely be required for this application. Possibilities include going to a Petrotape-style level measurement combined with a tilt sensor or using a submersible differential pressure-based level sensor that comes down through hatch. A thorough investigation of the maximum resolution limits for pressure-based sensors (or whatever sensor will be used for level detection), in conjunction with the data bus signal resolution.

One area in which future work should focus is in determining better sensors and/or mounting solution for switches, preferably coordinating with a manufacturer of tanker components to explore the possibility of getting switches built into the valve and hatch components. In addition, one limitation of this research was that the sensors gave one of only two status indicators: open or shut, with no "error" or "disconnected" indication. Thus, when problems were experienced, it was difficult to determine whether the issue was with a mounting solution, part failure, cable, PLC, etc. Such an error indicator may also improve the ability of the evidential reasoning system to place confidence in the readings. 
An additional area of sensor selection/development needed in future work is in regard to the level sensor; a reliable in-tank system should be identified or developed that can provide values at all times (whether the valve is open or closed) and provides readings in volume rather than simply pressure. It is anticipated that this will require some initial sensor calibration based on the compartment geometry, but this should be transparent to the in-cab equipment as it reads signals from the data bus.

The main development effort needed with the Fuel Marker sensor involves ruggedizing the sensor such that it could survive a series of rigorous environmental tests. Moving to a cast aluminum housing, as opposed to the plastic housing used in the POC test, is clearly a needed improvement that would not only waterproof the sensor but would also eliminate unwanted RF interference.

Furthermore, to better integrate the fuel marker sensor into the operation of the tanker vehicle, a mounting location on the truck that gives more continuous access to the stored fuel is desired. For example, if the fuel marker sensor could be integrated into the vehicle's tank, then it would be possible to monitor the fuel marker concentration at all times (not just when the valve was open and fuel was flowing). In addition, locating the sensor in the tank would eliminate possibility that the marker sensor-which was located at the sight glass for the field test—would interfere with the normal operation of the fuel tanker vehicle.

For Phase II of this project, the prototype sensor must be certified to permit installation and operation on a tanker vehicle not being operated on a controlled test track. This means certifying that the sensor is explosion proof and that it meets other regulatory requirements for operation on a fuel tanker vehicle; this will not only require a certified sensor but an approved or certified location and mounting method. Achieving this level of sensor maturity would likely require a commercial partner familiar with this process and willing to perform the necessary testing and design improvements required.

\subsubsection{Communications}

In the interest of moving toward standardization, the sensor interfaces may need to be reexamined. The inputs to the T2TCU in this phase of testing involved digital, analog, and RS485 interfaces with a set number of hard-wired inputs. The T2TCU should ultimately be designed to handle multiple compartments, and it may be that interfaces with the flexibility to handle multiple sensors (such as the RS485 marker interface) would be most appropriate for the final design.

The location of messages on the J1939 data bus is governed by Society of Automobile Engineers standards. Because this was a POC test and no standard message is currently defined for the signals needed for fuel payload monitoring, the Proprietary A message format was used. While this format was suitable for this preliminary testing, a fuel-tax-related message(s) must be standardized in order for this information to be readable by other monitoring devices. This will require decisions to be made as to the exact signals which may be present and the units and resolution for those signals while maintaining consistency with existing sensors and standards. The prescribed transmission rate must also be determined, taking into consideration the capacity of power line communications and the vehicle's J1939 data bus, as well as the required transmission rate to support the detection of various FTE scenarios. In addition, the message(s) must support a variable number of compartments while maintaining clarity as to which compartment(s) the posted readings are from. This effort will require cooperation between the standards committee(s), industry, and developers of the evidential reasoning system.

A next step in both the communications and evidential reasoning areas is the integration of a portion of the intelligence system into existing on-board telematics devices. Such devices handle vehicle-to-backoffice communications throughout the day to maintain up-to-date log information in a central back office 
and provide real-time alerts. The inclusion of much of this intelligence on board the vehicle would minimize the vehicle-to-BOS communication of FTE data to be mostly exception or alerts based.

A truck-to-BOS communications methodology that does not require constant communication with the BOS should be tested. This could involve backup satellite communications, monitoring of cell signal strength (with a notification message to the BOS as signal begins to drop off), and/or the resending of information that was not successfully transmitted (upon recovery of communications).

\subsubsection{Evidential Reasoning}

There are developments that need to be made in the evidential reasoning area in the second phase of this program. One key component is the implementation of a web-based geospatial (i.e., GIS) display of the terminal, retail outlets, and highway routes. This system would be used to track and validate vehicle and fuel locations against information on the shipping document. The GPS tracking device currently used by the partnering fleets would be used for this project. Second, an interface should be developed between a communications center and the fleet partner's shipping documentation system. Third, the evidential reasoning system should be designed based on the logic developed based on inputs from experts. Additionally, improvements are needed in methods used in the evaluation of marker concentration and fuel level changes. This effort would be closely linked with the improvement of the sensor suite.

For example, an alternative method for calculating the changes such as that of the fuel level variation could be to estimate the measurements $X_{t}$ at the beginning and at the end of intervals when the valve is open, as illustrated in Fig. 115. In this case, the changes in marker concentration and in the fuel level at each stop are calculated in three steps.

1. Calculate the median of two sample of data points within a small time period after the valve is open and right before the valve is closed.

2. Calculate the absolute change for each stop, $\Delta \mathrm{X}_{\mathrm{t}}=\mathrm{X}_{\mathrm{t} 2}-\mathrm{X}_{\mathrm{t} 1}$.

3. Calculate the relative change by comparing the current change $\Delta \mathrm{X}_{\mathrm{t}}$ against the reference value $\Delta \mathrm{X}_{0}$, according to the following equations $\mathrm{A}$ and $\mathrm{B}$.

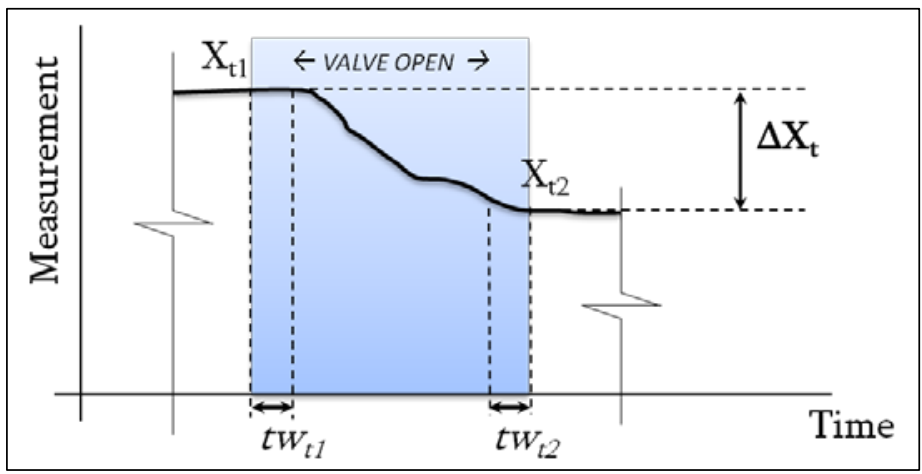

Fig. 115. Portion Fuel Level Profile at Stop t.

For a non-scheduled stop, the observed change would be

$$
\Delta \mathrm{X}=\left|\Delta \mathrm{X}_{\mathrm{t}}\right| / \Delta \mathrm{X}_{0}
$$


For a scheduled stop, the observed change would be

$$
\Delta \mathrm{X}=\left|\Delta \mathrm{X}_{0}+\Delta \mathrm{X}_{\mathrm{t}}\right| / \Delta \mathrm{X}_{0}
$$

\subsection{LONG-TERM EFFORTS AND APPLICATIONS}

In the recommended system architecture called out in Sect. 8.1, it is propose to deploy a system in which most of the data monitoring and process is done on board the vehicle to reduce the data communications cost and reduce the complexity of the central system. This recommended architecture and the associated onboard electronics would need to be tested to determine if accurate and responsive exception-based alerts could be process as well as the validation of a localized geolocation system.

There is a need for real-world testing to validate the concept, the hardware, and the FTE Upload Data Service software, as well as the usage and acceptance to stakeholders. This need must be balanced relative to timing with the need to mature the hardware and software to be able to effectively conduct real-world testing. With this said, an intermediate testing step may be required to validate hardware and software maturity as well as trailer hardware and electronics mounting solutions before committing to the expense or real-world testing.

A particularly important component to any future FTE research efforts is a cost-benefit analysis. In order for such any developed system to be feasible and accepted by industry and enforcement, there must be a financial case for such a system. As such, significant incentives will likely be needed for fleet participation.

An additional area in which research will need to be performed along with maturation of hardware is use of the data. Part of a large-scale deployment such as might be conducted in a field operational test should include studying how enforcement might make use of the data. In addition, there may also be a military application for fuel transfer to be explored. The cost-benefit analysis may show certain applications to be more appropriate than others, and future research should focus on these applications.

Lastly, there are hybrids of the proposed system that, while not providing the full complement of information, could provide information useful in fuel thief detection and post-fuel-delivery audit. For example, a hybrid system without the fuel marker component would still register the fuel loading and unloading events location and time, as well as all the hatch opening events. The benefit of these hybrid systems is cost saving relative to vehicle-borne technology as well the cost to mark the fuel. The value of these hybrid systems should be explored to determine if a less complex system is capable of providing similar value towards the prevention and detection of FTE. 


\section{REFERENCES}

1. Wiley Online Library, Kirk-Othmer Encyclopedia of Chemical Technology: Naphthenic Acids, doi: 10.1002/0471238961, published online December 4, 2000, pp. 1-10.

2. W. Boyes, "First the application, then the product," ControlGlobal.com [Online], February 1, 2007 [cited: May 29, 2011] http://www.controlglobal.com/articles/2007/022.html.

3. J. Hunter, Civacon Corporation, Kansas City, Missouri, private communication, 2011.

4. “Liquid level monitor uses guided-wave radar,” Bulk Transporter Magazine [Online], April 1, 2003 [cited: November 10, 2010]. .http://bulktransporter.com/mag/transportation_liquid_level_monitor/.

5. Petrotape Level Gauge for Petroleum Products,” JOWA USA, Littleton, Mass., November 2009.

6. P. Valin, "Reasoning Frameworks for Fusion of Imaging and Non-imaging Sensor Information,” in Proceedings of the Workshop on Estimation, Tracking and Fusion: A Tribute to Yaakov Bar-Shalom, Naval Postgraduate School, Monterey, Calif., May 17, 2001, pp. 269-282.

7. J-B. Yang and D-L. Xu, "On the Evidential Reasoning Algorithm for Multiple Attribute Decision Analysis under Uncertainty," IEEE Transactions on Systems, Man, and Cybernetics-Part A: Systems and Humans, Vol. 32, No. 3, May 2002.

8. Y. Tang and E. Al-shaeer, "Reasoning about Uncertainty for Overlay Fault Diagnosis Based on EndUser Observations,” 2009, http://www.arc.uncc.edu/pubs/faultdiag-infocom09.pdf.

9. U.S. Energy Information Administration, Annual Energy Review 2010, www.eia.gov/aer. 



\section{APPENDIX A. FLUORESCENCE SPECTRA}

The following figures show fluorescence spectra collected for pump diesel containing the fielded fuel dye Solvent Red 26, Sudan Orange II, and Solvent Blue 59 to determine whether competing fluorescence in the wavelength region of excitation above $630 \mathrm{~nm}$ would pose any significant interference, and none was noted.

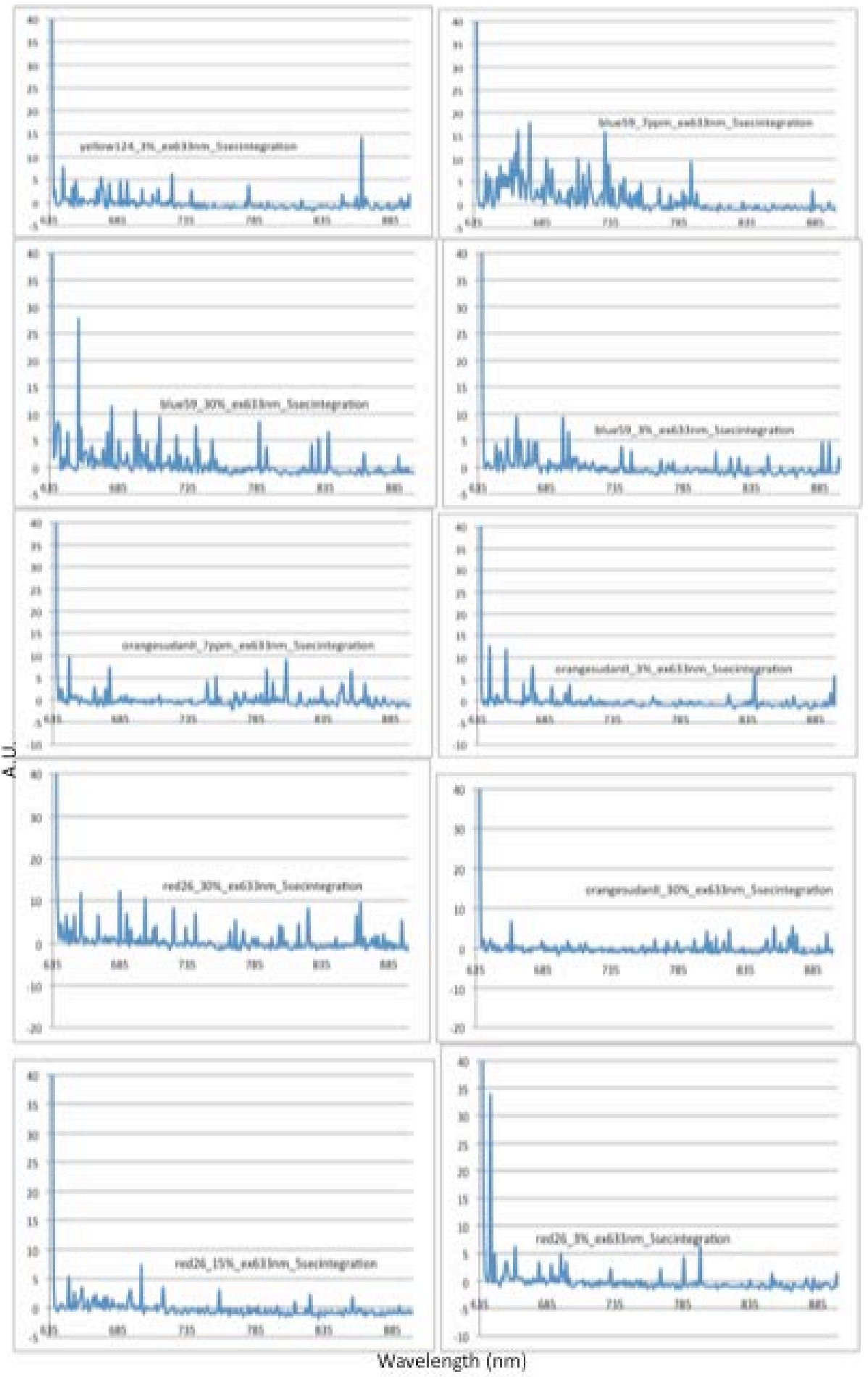



APPENDIX B. FUEL MARKER SENSOR SCHEMATICS AND LAYOUT

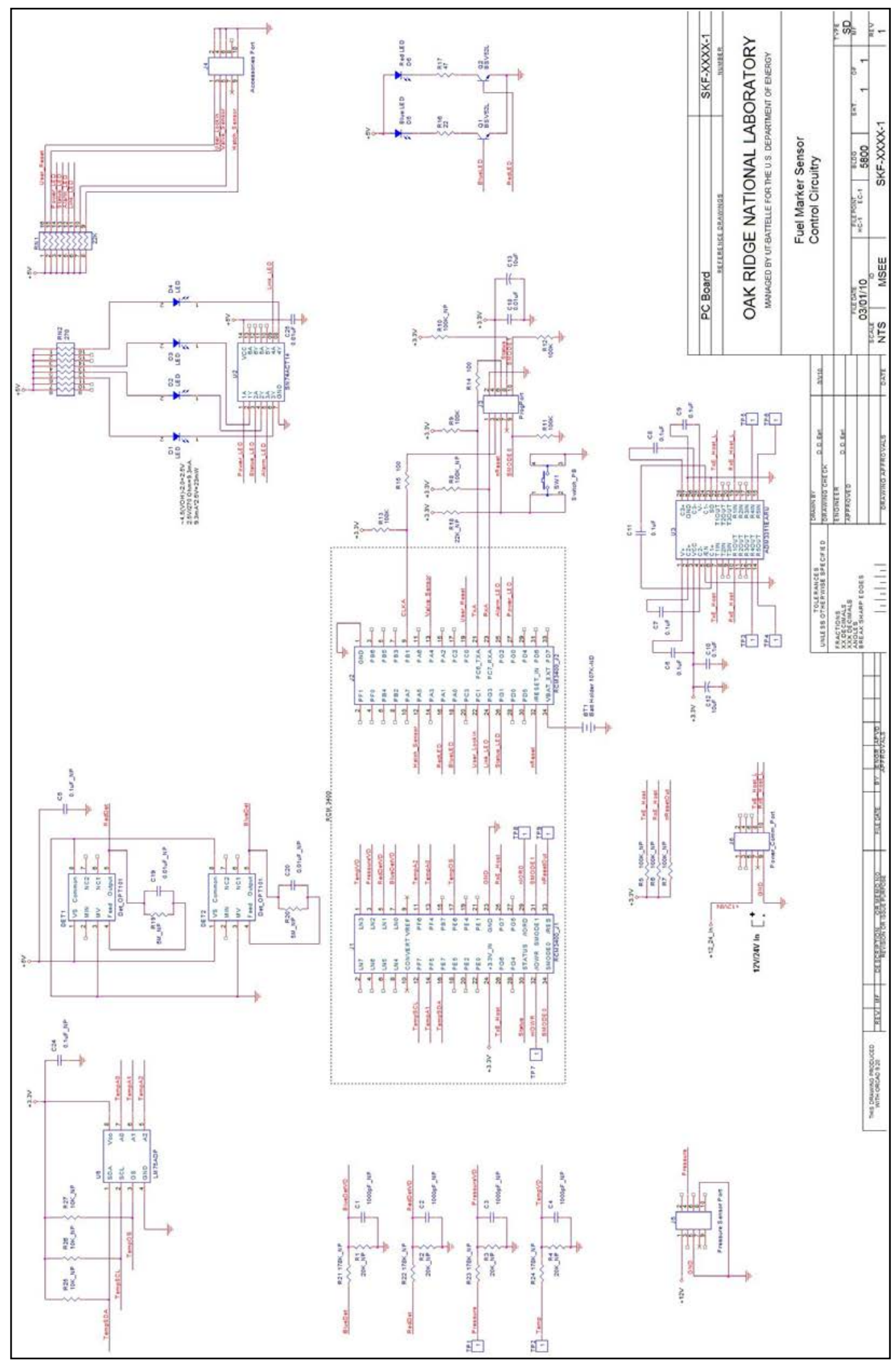

B-1 


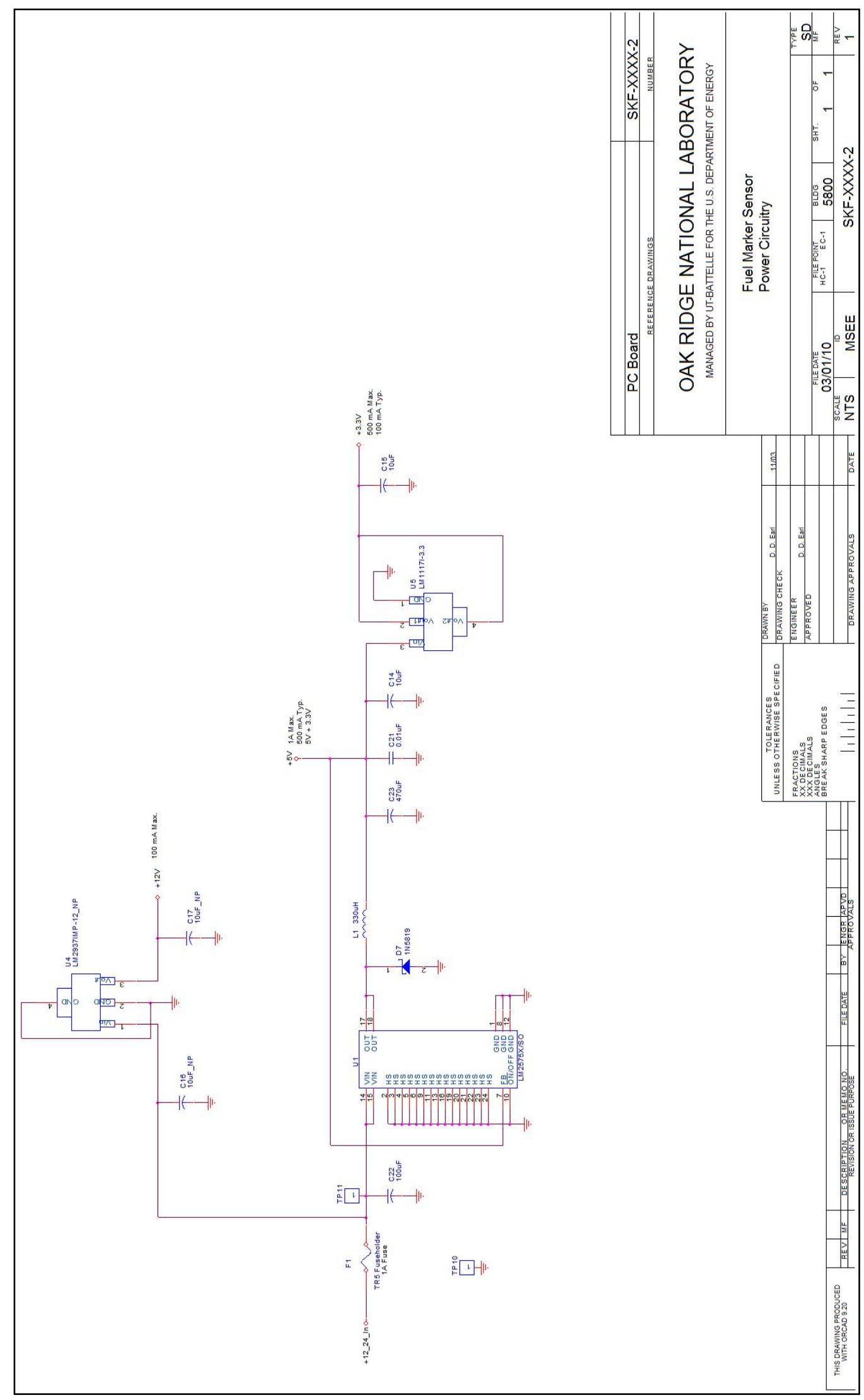

B-2 


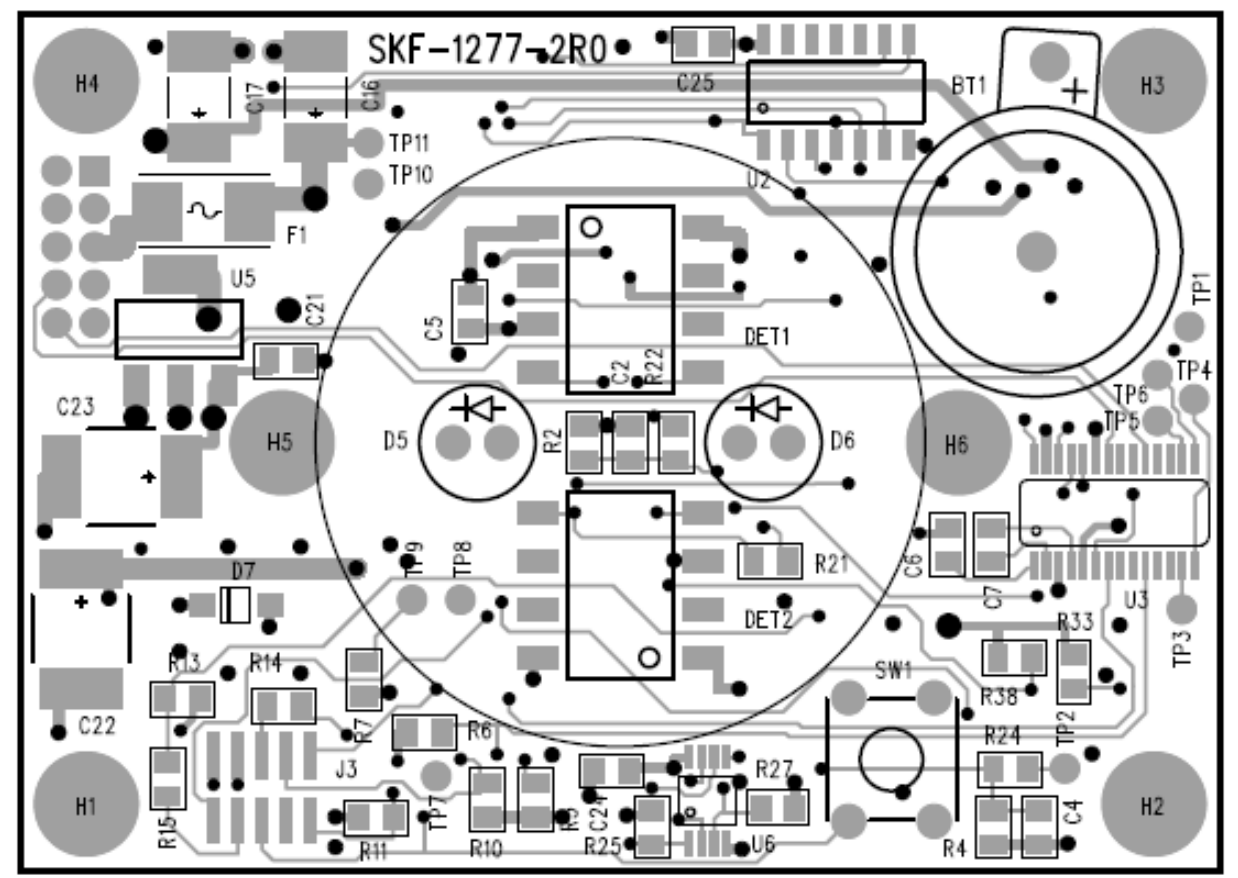

TOP SIDE SILKSCREEN

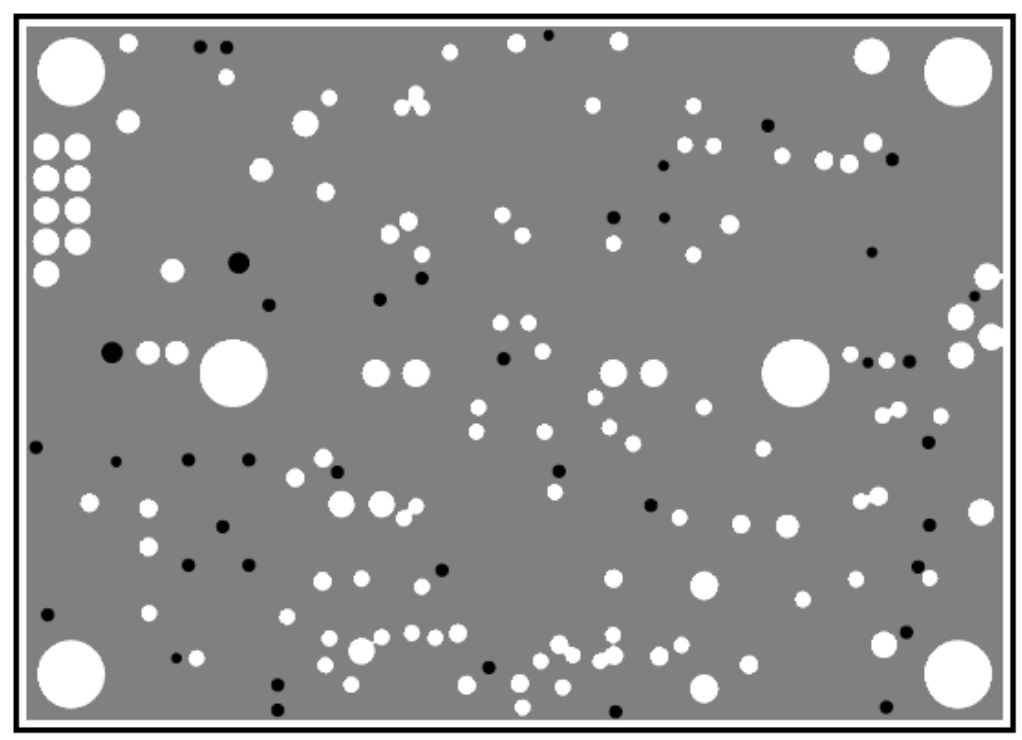

INNER LAYER 2 


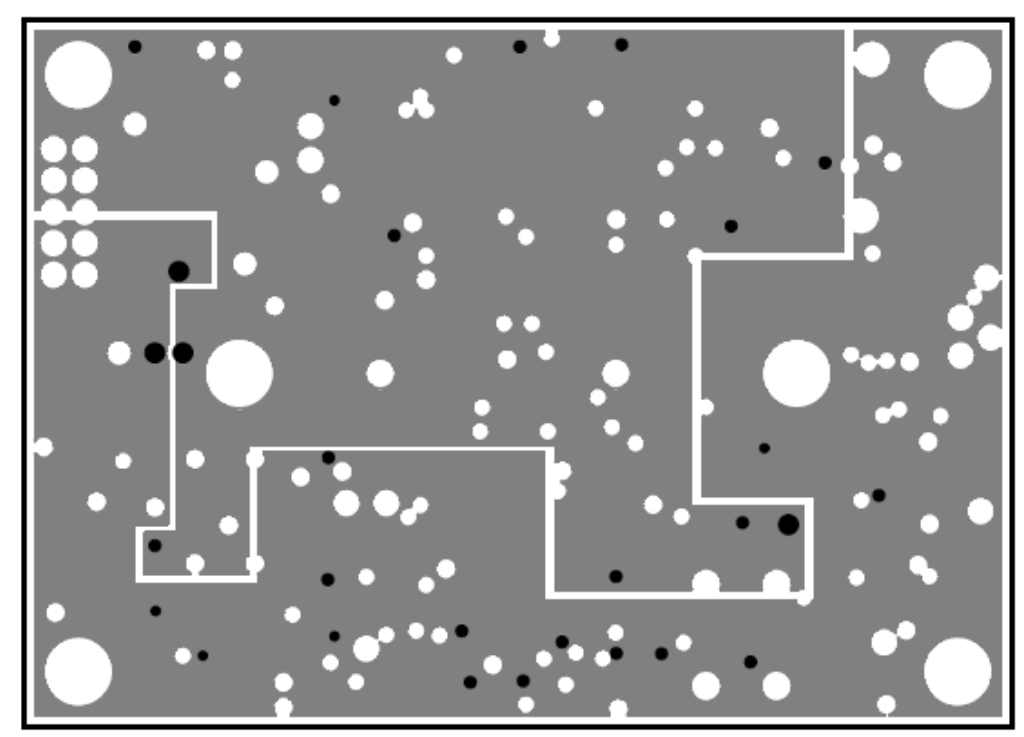

INNER LAYER 3

$-\phi$

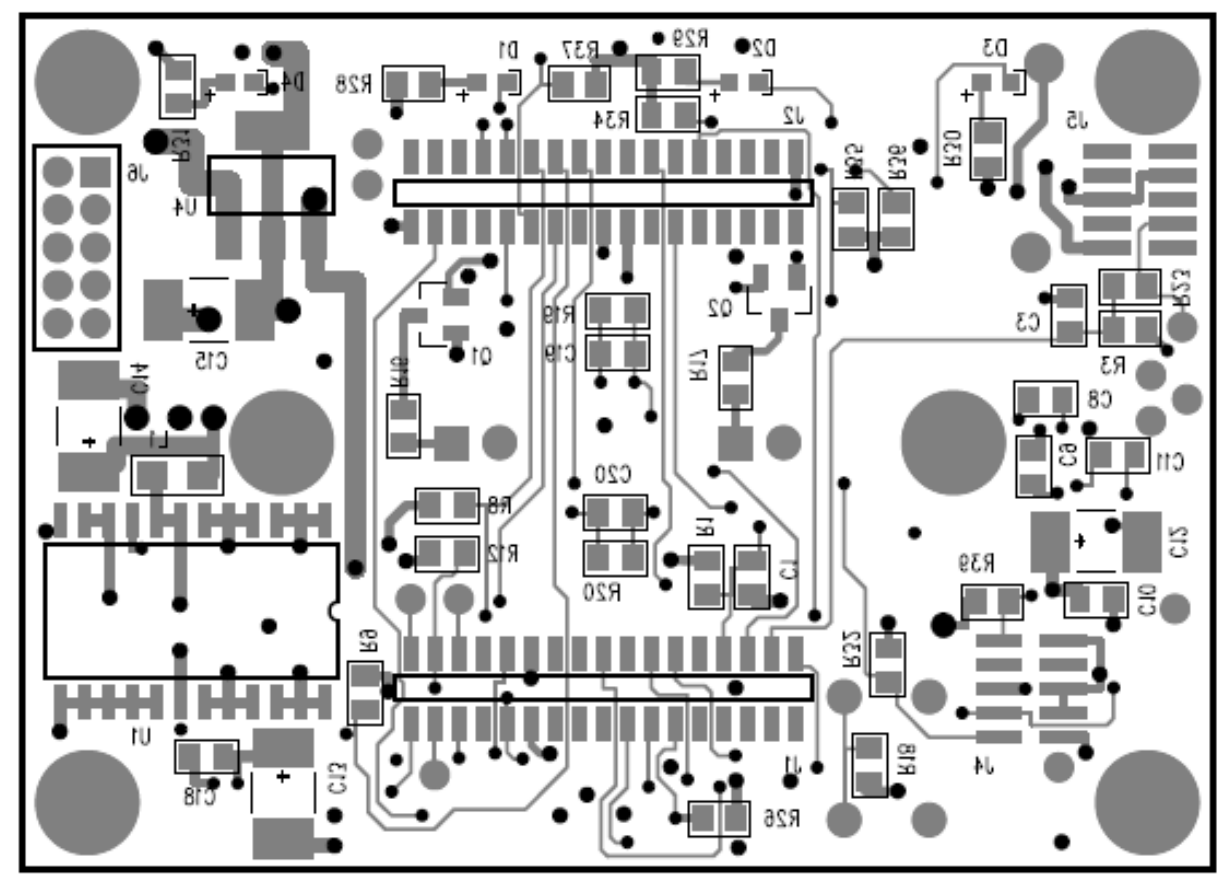

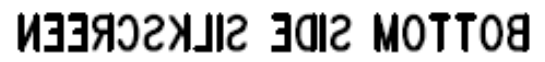

(क) 


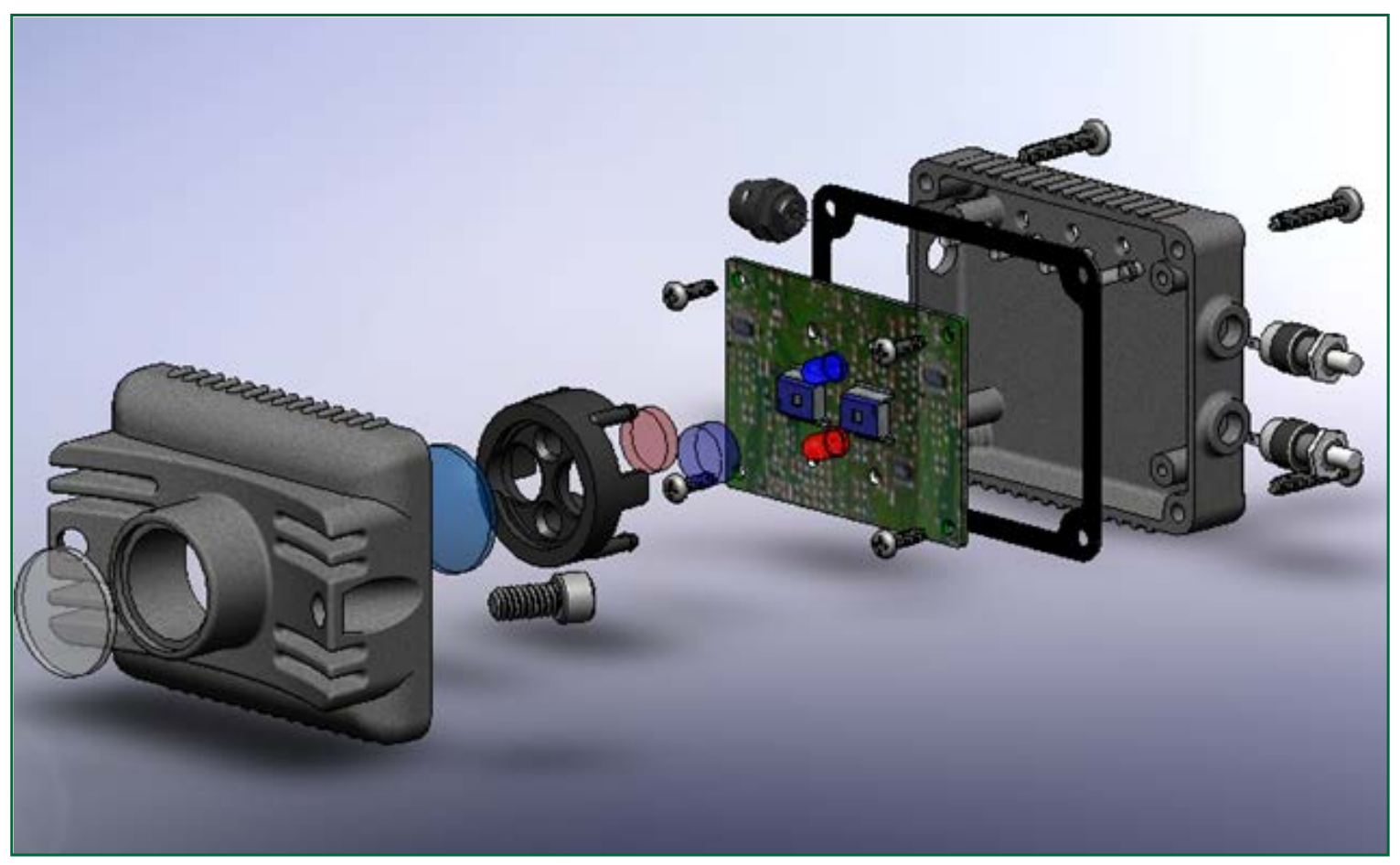

Fig. C.1. Assembly of sensor components.

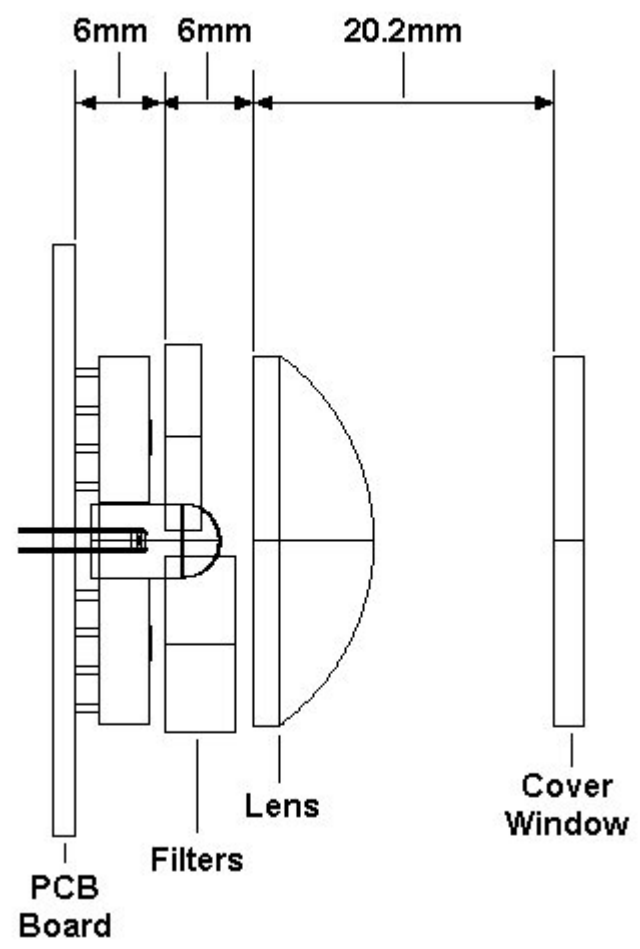

Fig. C.2. Side view of critical dimensions. 


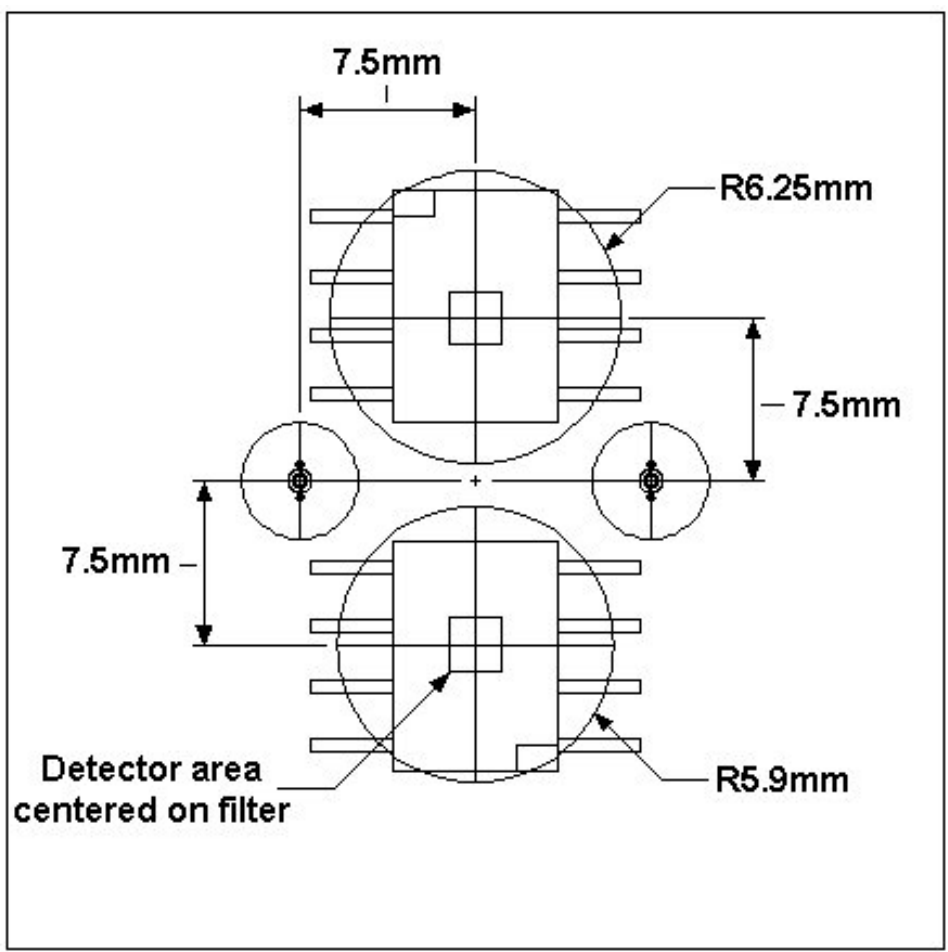

Fig. C.3. Top view of critical dimensions.

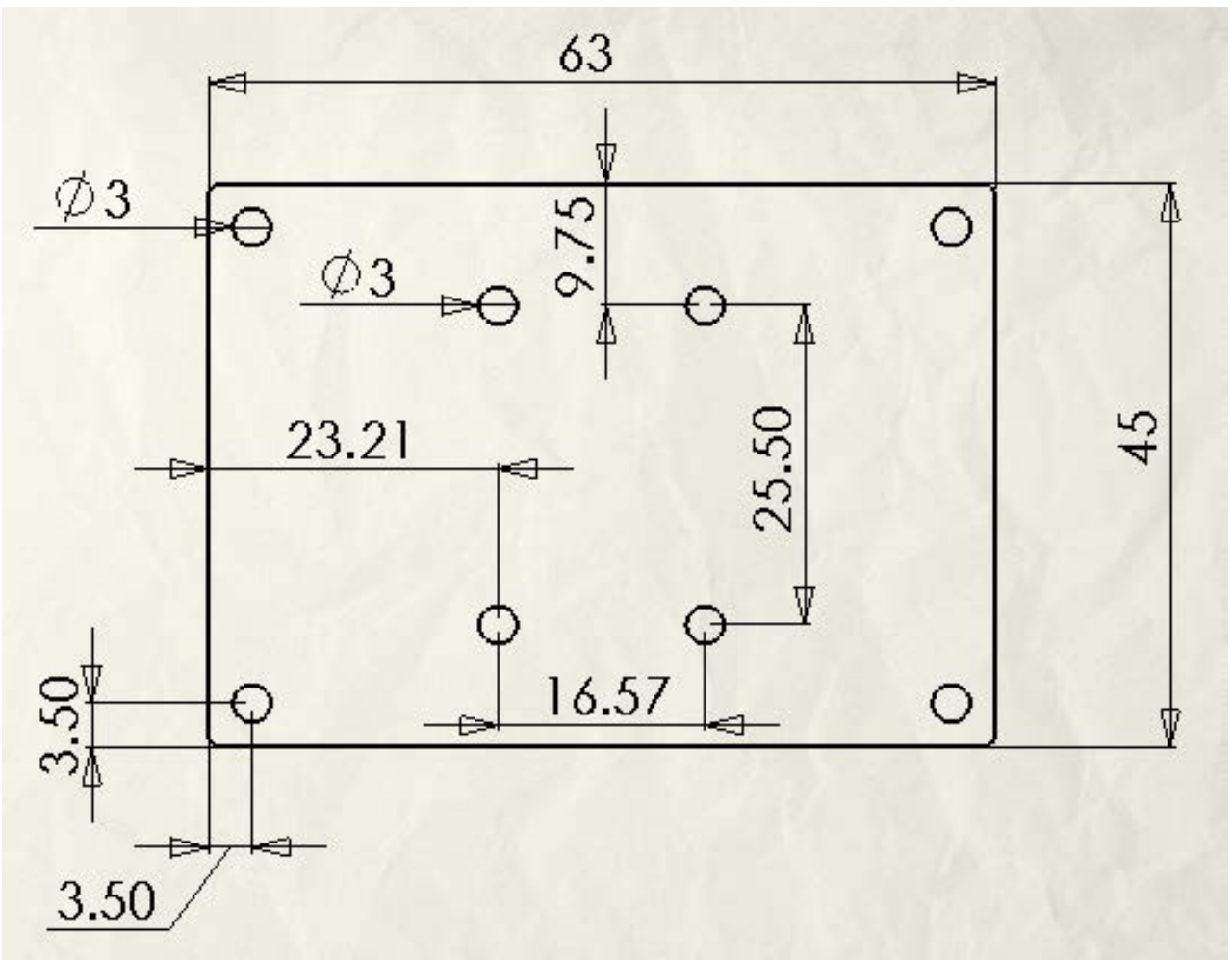

Fig. C.4. Critical holes and edges on PCB board layout. 


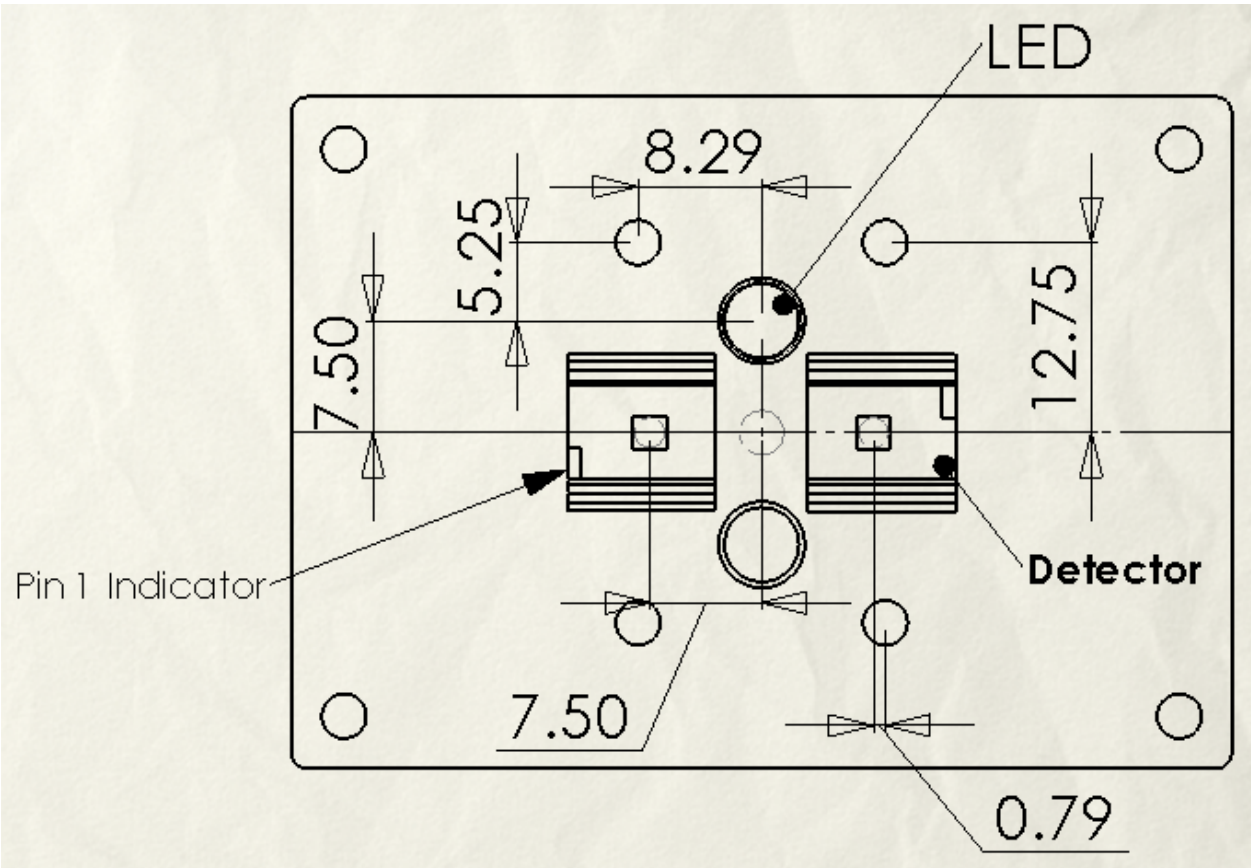

Fig. C.5. Critical PCB component placement (front).

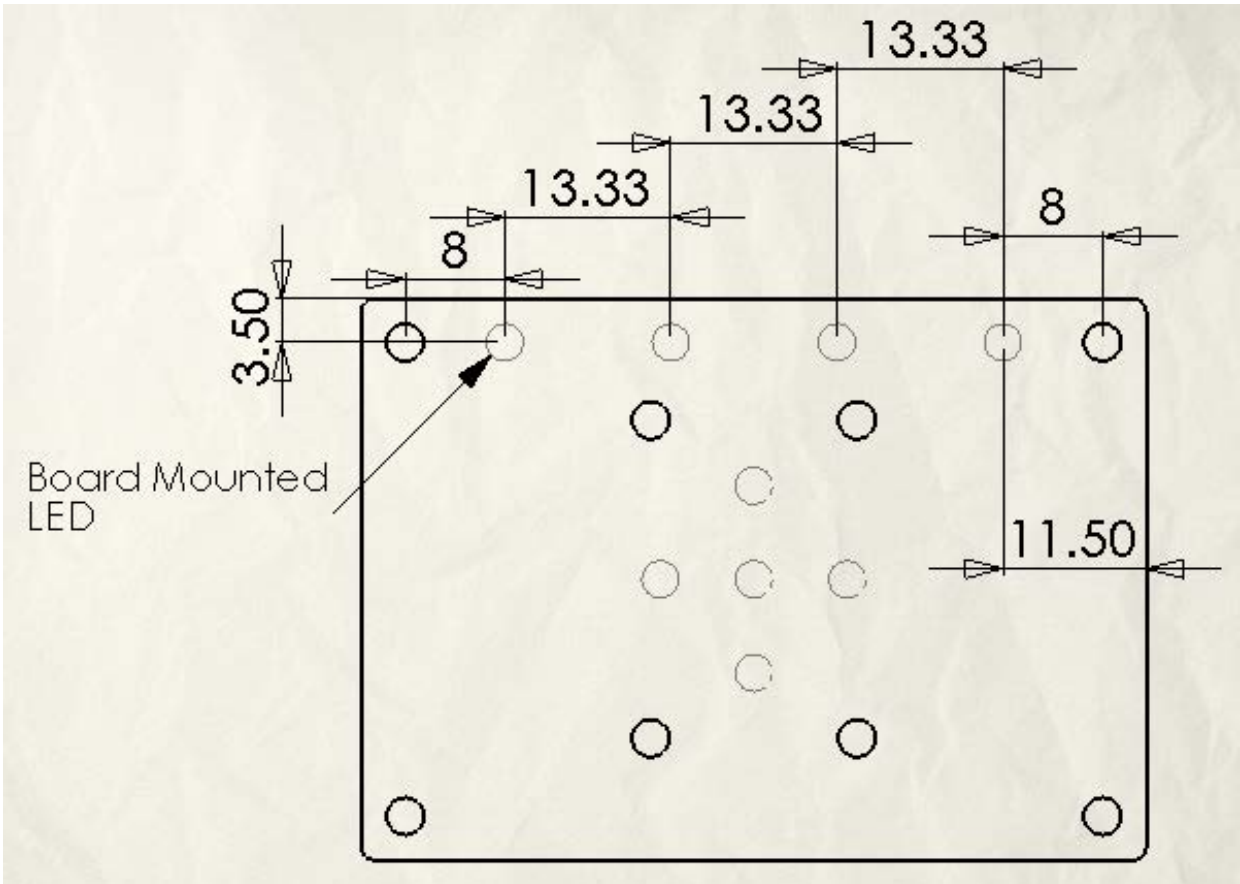

Fig. C.6. Critical PCB component placement (back). 



\section{APPENDIX D. FUEL MARKER SENSOR BILL OF MATERIALS}

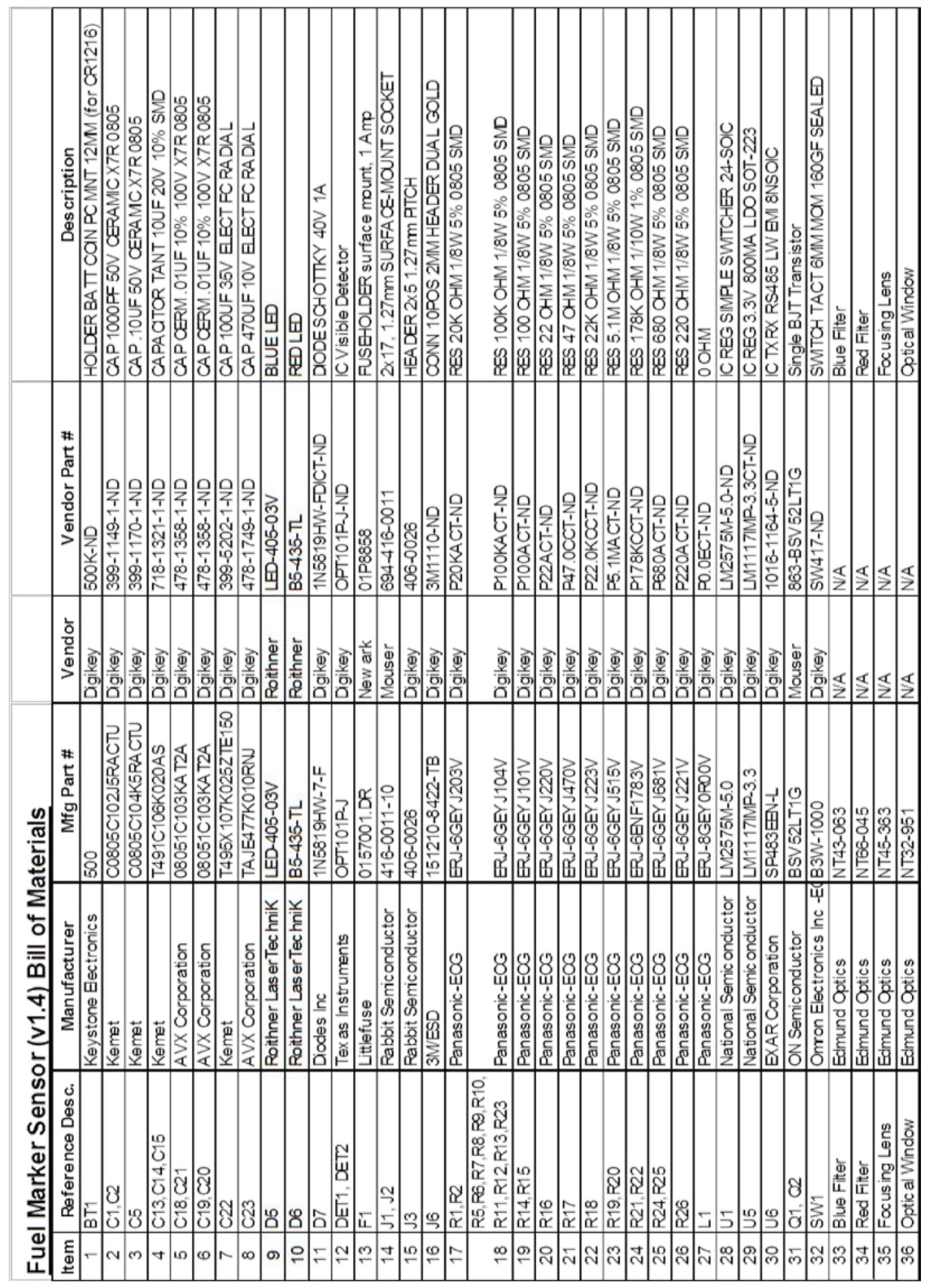

D-1 



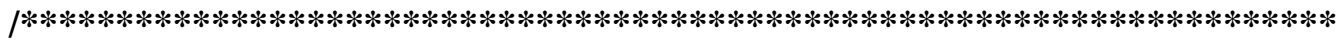

FTC_Main.c

Duncan Earl, 2011

This program is for the Rabbit Semiconductor RCM3400 series controllers And prototype ORNL Fuel Marker Sensor Prototype

Description

This program turns a red and blue LED on and off while acquiring the signal from two filtered photodetectors. The LEDs illuminate a sample under test and the fluorescence returns a signal in two distinct wavelength bands. The ratio of the filtered detector outputs is an indicator of a marker concentration in the sample.

The program communicates over an RS485 serial link to relay data and to receive instructions from a host computer.

The programmed pins for all inputs and outputs are shown below:

Blue LED = PA0

Red LED $=$ PA1

Blue Detector Input $=$ LNO $($ AI0)

Red Detector Input $=$ LN1 (AI1)

This program uses TxD (PC0), RxD (PC1) and PD4 for transmit enable.

Version Notes:

v0.01 - First implementation

v0.02 - Added RS232 Communication Capabilities

v0.03 - Converted to RS485 Communication Capabilities

v0.04 - Converted to RS485 Hardware

v0.05 - Corrected Rx/Tx issue on RS485

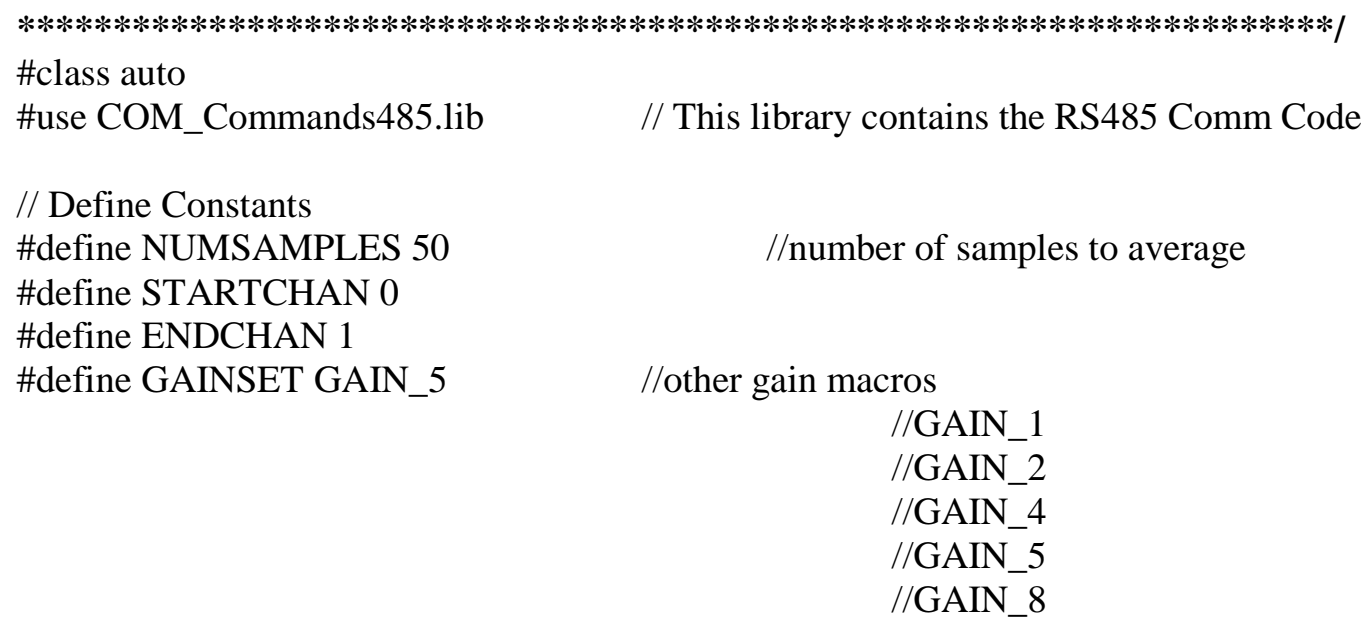


\#define DS1 6

\#define DS2 7

\#define ON 0

\#define OFF 1

\#define CH_ESCAPE 27

\#define E_BAUDRATE19200L

// Setup Chip A/D Conversion Resolution

\#define ADC_RESOLUTION 12

// RS485 communications

\#define ser485open serDopen

\#define ser485close serDclose

\#define ser485wrFlush serDwrFlush

\#define ser485rdFlush serDrdFlush

\#define ser485putc serDputc

\#define ser485getc serDgetc

//////

// change serial buffer name and size here

// for example: DINBUFSIZE to CINBUFSIZE

//IIII

\#define DINBUFSIZE 15

\#define DOUTBUFSIZE 15

////I/

// change serial baud rate here

I/I/I/I

\#ifndef_485BAUD

\#define_485BAUD 19200

\#endif

nodebug

void ser485Tx( void )

\{

\}

BitWrPortI(PDDR, \&PDDRShadow, 1, 4); // set port D bit 4 high
//GAIN_10

//GAIN_16

//GAIN_20

//port D bit 6

//port D bit 7

//state to turn on led

//state to turn off led

\section{/*}

Disables the RS485 transmitter.

This puts the device in listen mode which allows to receive data from the RS485 interface.

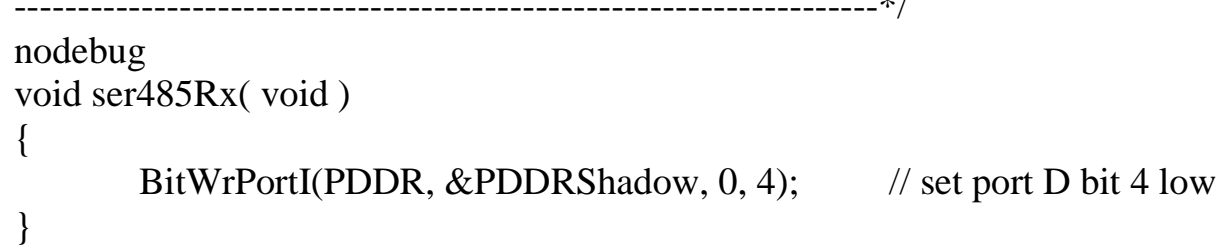


// set the STDIO cursor location and display a string

void DispStr(int $\mathrm{x}$, int $\mathrm{y}$, char *s)

\{

$\mathrm{x}+=0 \times 20$

$\mathrm{y}+=0 \times 20$

printf ("\x1B=\%c\%c\%s", $\mathrm{x}, \mathrm{y}, \mathrm{s})$;

\}

// read the A/D with using the low level driver

//nodebug (unsigned int -> changed to float)

double sample_ad(int channel, int num_samples)

\{

auto unsigned long rawdata;

auto unsigned int sample;

auto unsigned int cmd;

//convert channel and gain to ADS7870 format

$/ /$ in a direct mode

cmd $=0 \times 80 \mid($ GAINSET $* 16+($ channel $\mid 0 \times 08))$;

for (rawdata $=0$, sample=num_samples; sample $>0$; sample--)

\{

// execute low level A/D driver

\}

rawdata $+=$ (long) anaInDriver(cmd, ADC_RESOLUTION);

\}

return ((double) (rawdata/num_samples));

//changed float to double, changed int to double

double convert_volt(int channel, double value)

\{

//changed auto float to double

double voltage;

// convert the averaged samples to a voltage

voltage $=($ value -

_adcCalibS[channel][GAINSET].offset)*(_adcCalibS[channel][GAINSET].kconst);

\}

return voltage;

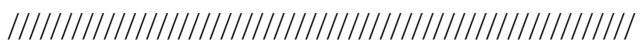

// RED LED on prototype is controlled by port A bit 1

$/ /$ turns on if state $=1$

$/ /$ turns off if state $=0$

///////////////////////////////////////////////////

void RedLED(int state)

\{

if (state $==\mathrm{ON})$

\{

BitWrPortI(PADR, \&PADRShadow, 1, 1); //reversed for use with laser 


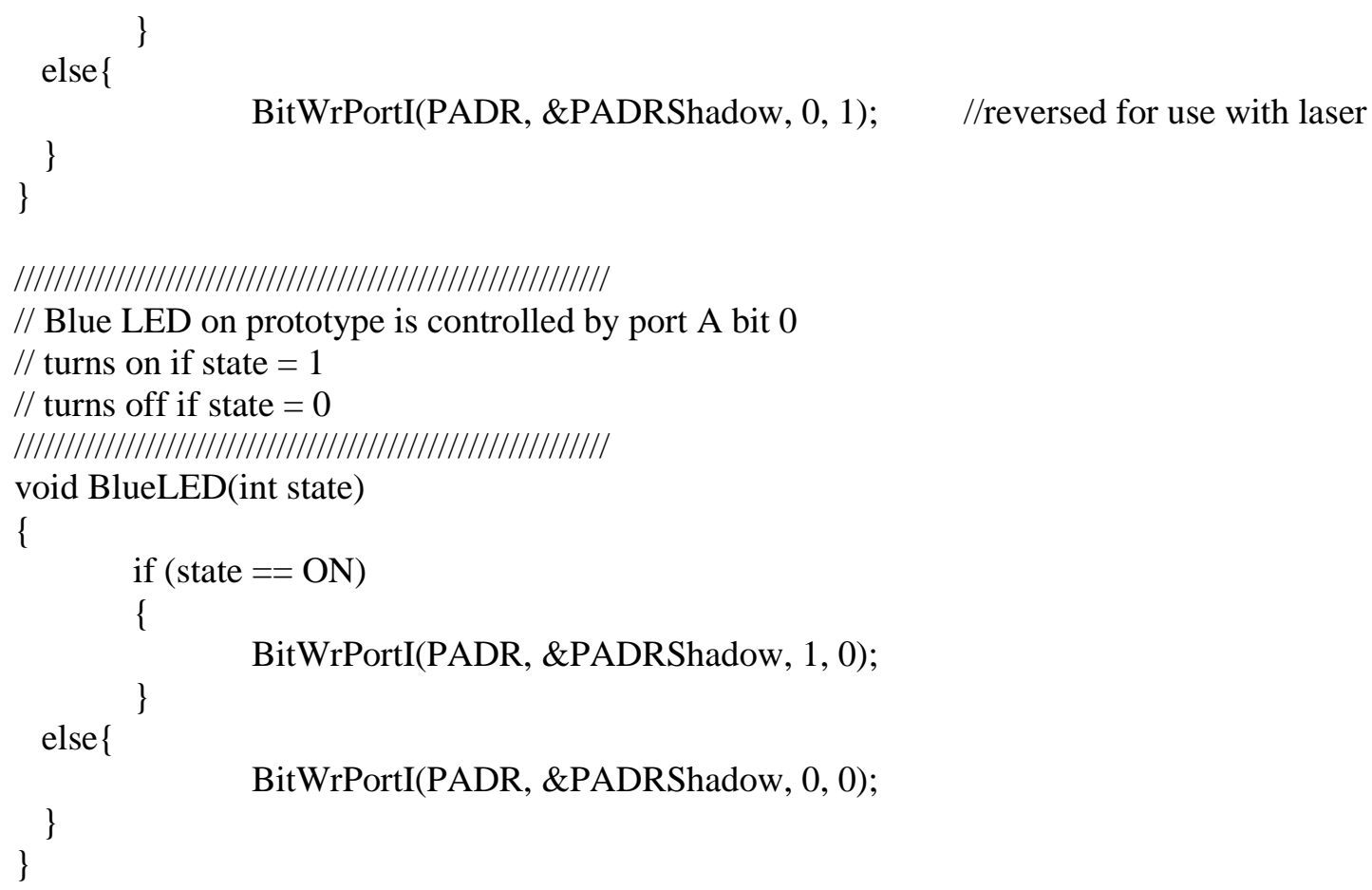




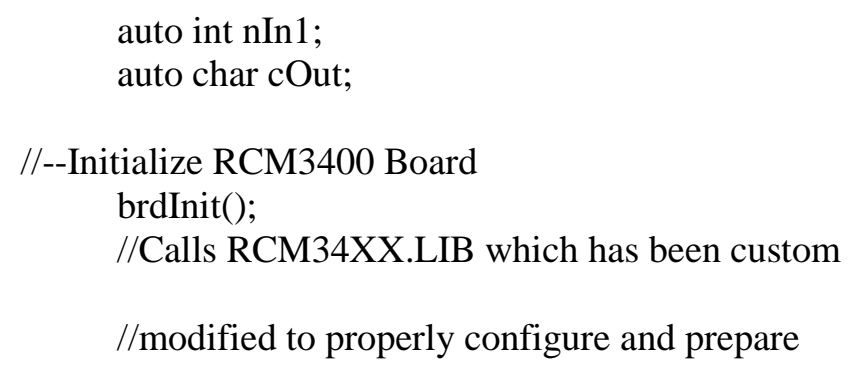


background

avg_sample = sample_ad(channel, NUMSAMPLES);

//Acquire Average Signal

ad_background[channel] = convert_volt(channel, avg_sample); //Store as

\}

//--Step 2: Acquire Blue Fluorescence Levels

BlueLED(ON);

RedLED(OFF);

//Turn On Blue LED

waitfor( DelayMs( 100 ) ); //Wait 100 milliseconds to allow LEDs to adjust

channel $=0$;

avg_sample = sample_ad(channel, NUMSAMPLES); $\quad$ //Acquire average blue detector voltage ad_inputs[channel] = convert_volt(channel, avg_sample); //Store signal

BlueLED(OFF); $\quad$ //Turn off Blue LED

RedLED(OFF);

waitfor( DelayMs( 100 ) ); //Wait 100 milliseconds to allow LEDs to adjust

//--Step 3: Acquire Red Fluorescence Levels

BlueLED(OFF); $\quad$ //Turn off Blue LED

RedLED(ON);

//Turn on Red LED

waitfor( DelayMs( 100 ) ); //Wait 100 milliseconds to allow LEDs to adjust

channel $=1$;

avg_sample = sample_ad(channel, NUMSAMPLES);

//Acquire average red detector voltage

ad_inputs[channel] = convert_volt(channel, avg_sample); //Store signal

//--Step 4: Turn Off LEDs and Compute Results

BlueLED(OFF);

RedLED(OFF);

//Turn off Blue LED

//Turn off Red LED

if ((ad_inputs[0]-ad_background[0])! $=0)\{$ ratio $=($ ad_inputs[1]-ad_background[1] $) /($ ad_inputs[0]-ad_background[0] $) / 1$; //2.523625;

///1.553;

\}

else

\{

ratio $=0.0$

\}

//--Step 5: Store Results

wfd StoreVariables(ad_inputs[0]-ad_background[0], ad_inputs[1]-ad_background[1],ratio, 0);

//--Step 6: Display Results (FOR DEBUG PURPOSES ONLY)

display[0] = '10';

channel $=0$;

sprintf(s, "\t\%6.3f", ad_background[channel]);

strcat(display, s); 


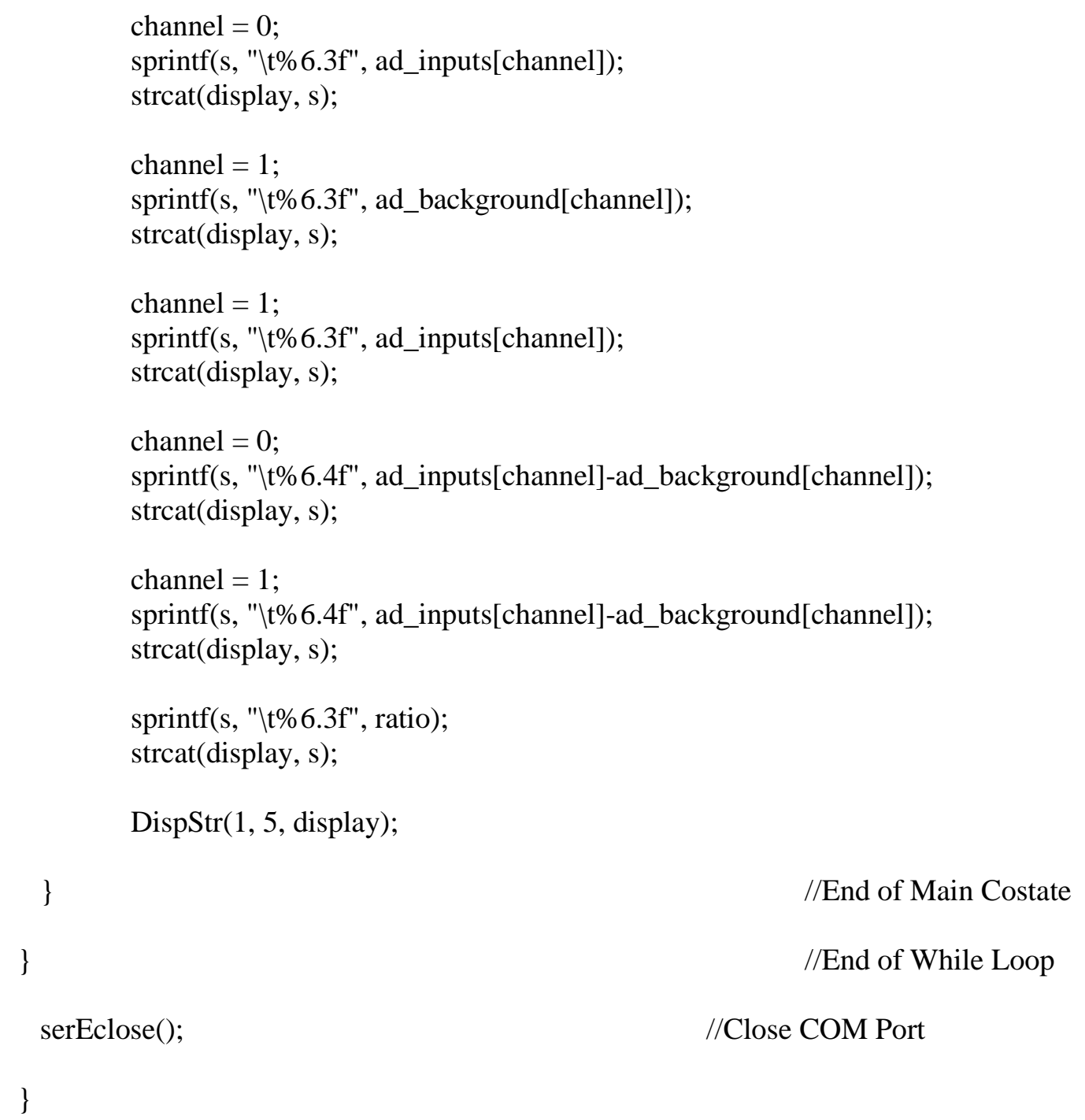




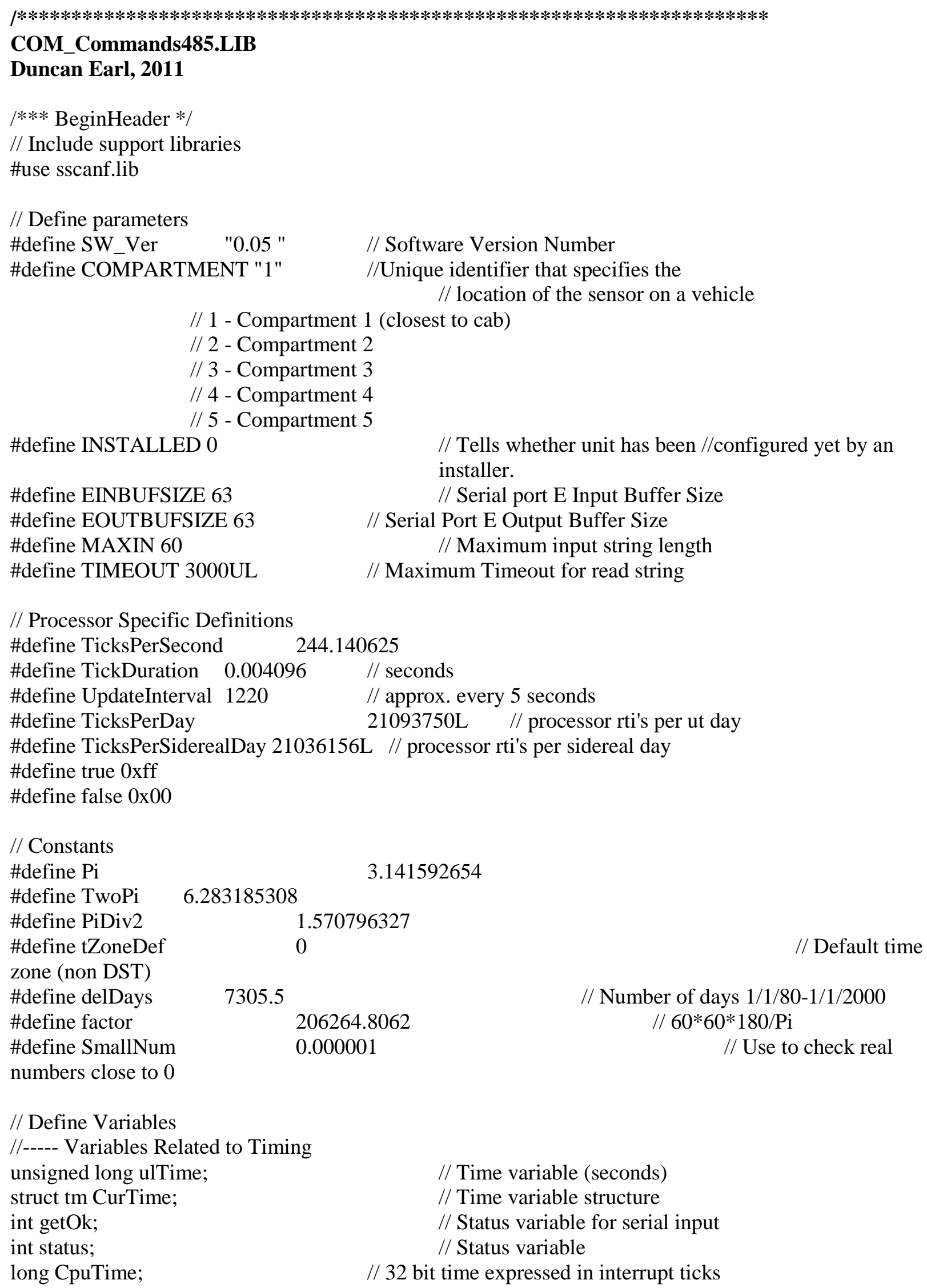


byte Day;

byte Month;

int Year;

short Hour;

short Minute;

short Second;

//------ Variables Related to String Parsing

char instr[MAXIN+1];

char out_str[32];

char CmdType;

char StopFlag;

char QFlag;

char OffSunFlag;

char StowFlag;

long qd_count[2];

char qd_errorflags[2];

char qd_isrflag;

//------- Variables related to stored parameters

double local_reddet;

double local_bluedet;

double local_ratio;

int local_alarm;

double init_ratio;

char id[33];

word SerialNumber;

/*** EndHeader */

/*** Beginheader StoreVariables */

cofunc StoreVariables(double sv1, double sv2, double sv3, int sv4);

/*** endheader */

/* START FUNCTION DESCRIPTION

StoreVariables

$<$ COM_Commands.LIB $>$

SYNTAX: $\quad$ cofunc StoreVariables(int *ratio, int *alarm);

DESCRIPTION: $\quad$ Receives key variables and stores them.

PARAMETER1: double reddet

PARAMETER2: double bluedet

PARAMETER3: double ratio

PARAMETER1: int alarm

RETURN VALUE: (none)

END DESCRIPTION

cofunc StoreVariables(double sv1, double sv2, double sv3, int sv4) // Handle incoming commands from host
// Curent Day

// Current Month

// Current Year

// Curent Hour

// Current Minute

// Current Second

// Input string for RS-232 commands

// Output string for RS-232 status

// Command type

// Stop Flag

// Query Flag

// Off-Sun Request to Host Flag

// Stow Request to Host Flag 


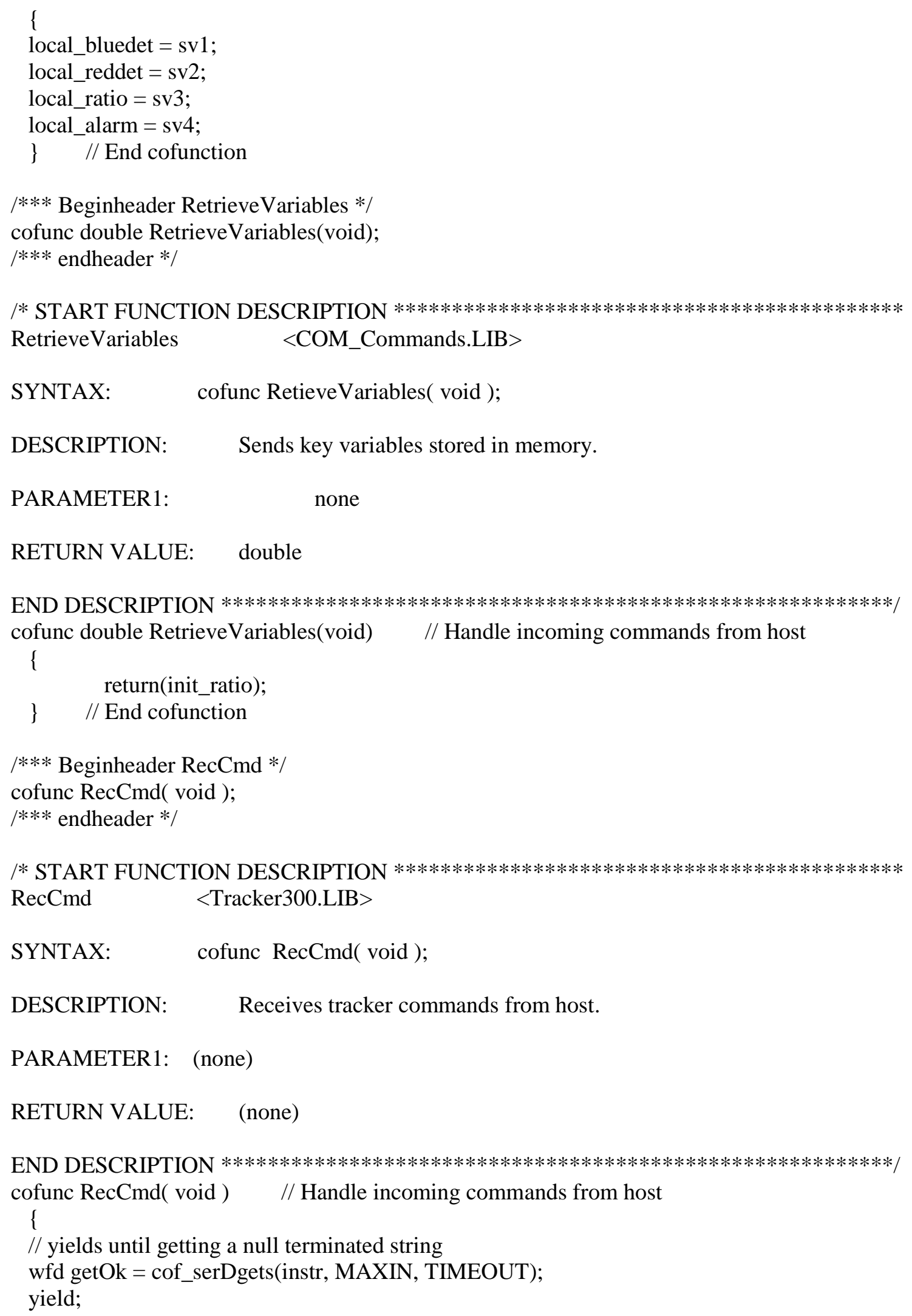


if (!getOk) abort;

yield;

sprintf(\&CmdType, "\%s",\&instr[0]);

yield; $\quad$ // Only allow Stop and Pos Cmd while moving

// Send string for parsing

ProcessCommand(instr);

\} // End receive commands cofunction

/*** BeginHeader msDelay2 */

void msDelay2(unsigned int delay)

\{

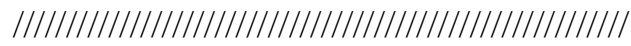

// Waits for $\mathrm{N}$ milliseconds of delay

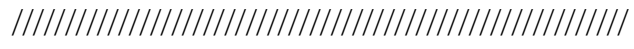

auto unsigned long done_time;

done_time = MS_TIMER + delay;

\}

while( (long) (MS_TIMER - done_time) $<0$ );

/*** EndHeader */

/*** BeginHeader ConsoleHelp */

void ConsoleHelp(void);

/*** EndHeader */

//Print help list showing commands

void ConsoleHelp(void) \{

// Enable transmitter

BitWrPortI(PDDR, \&PDDRShadow, 1, 4); // set port D bit 4 high

sprintf(out_str,"Commands-\r|n");

serDputs(out_str);

sprintf(out_str,"get [ratio|bluedet|reddet] -- print detector values\r\n");

serDputs(out_str);

sprintf(out_str,"get [date|ut|id|sernum|firmware] -- get setup info\r\n");

serDputs(out_str);

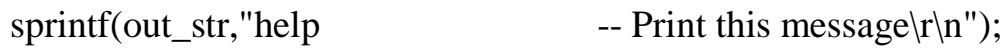

serDputs(out_str);

sprintf(out_str,"set date $<\mathrm{mm} / \mathrm{dd} /$ yyyy $>\quad$-- set date value $\backslash r \mid n ") ;$

serDputs(out_str);

sprintf(out_str,"set ut <hh:mm:ss.sss> $\quad$-- set GMT time value $>r \backslash n ")$;

serDputs(out_str);

sprintf(out_str,"set [sernum|id|adr] < val> $\quad$-- establish new configuration values $\backslash r \backslash n ") ;$

serDputs(out_str);

// Disable transmitter

msDelay2(500); //Wait 500 milliseconds to allow RS485 Signal to Complete

BitWrPortI(PDDR, \&PDDRShadow, 0, 4); // set port D bit 4 low 


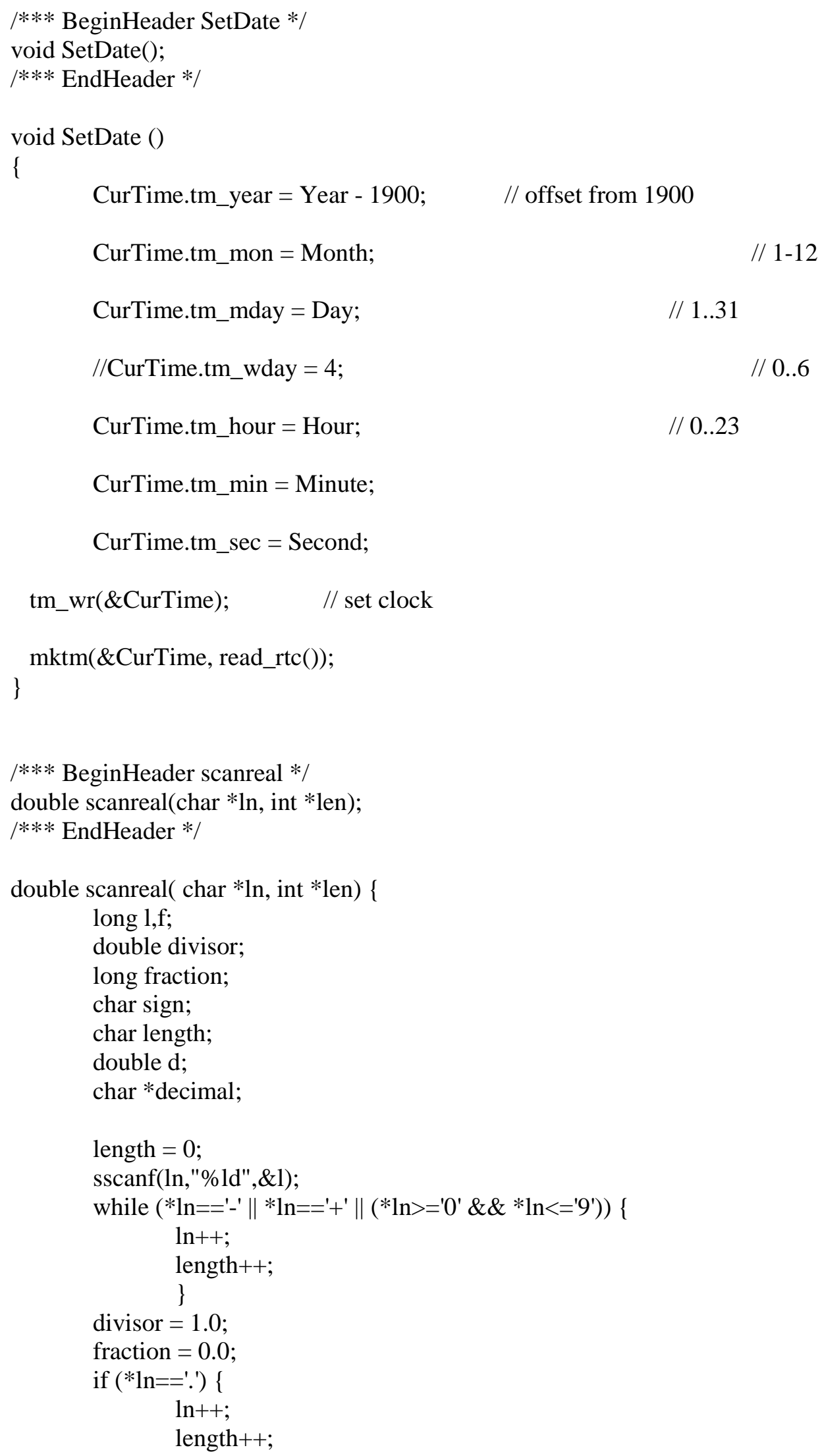




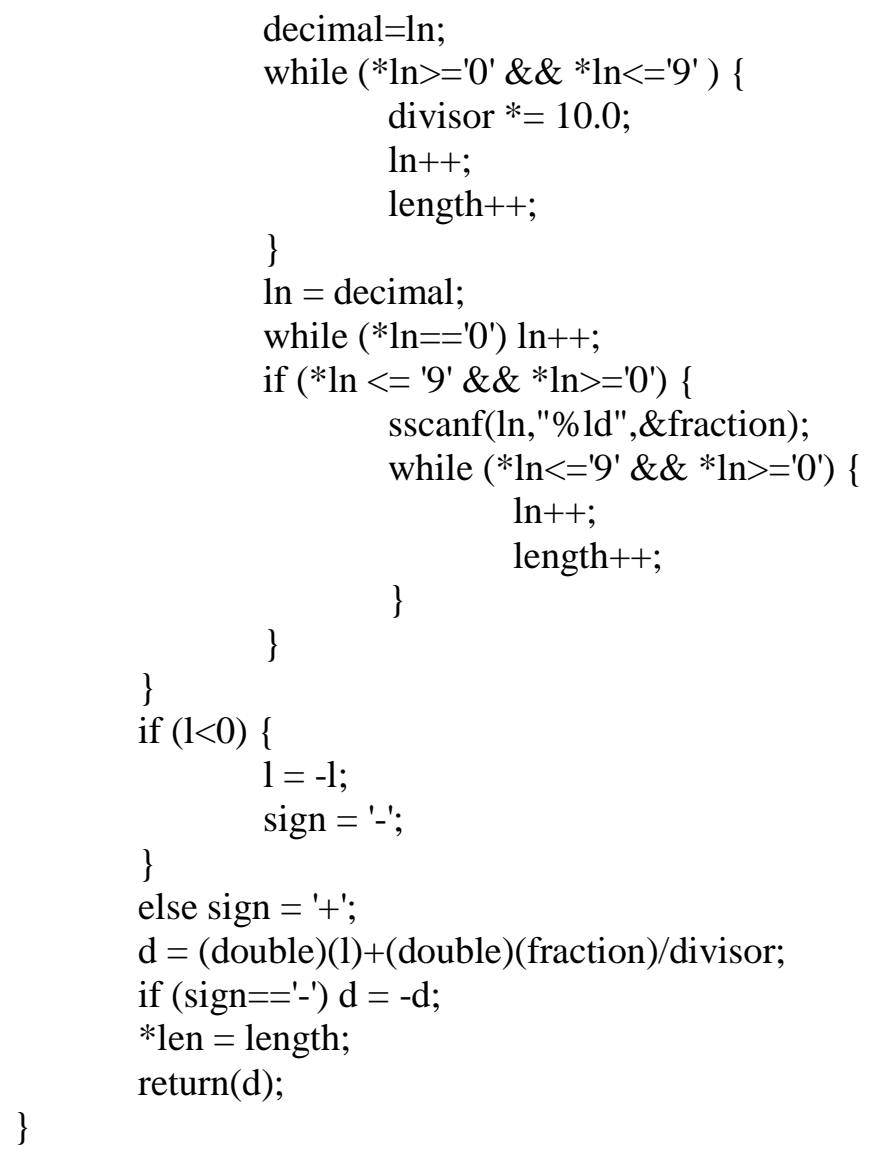




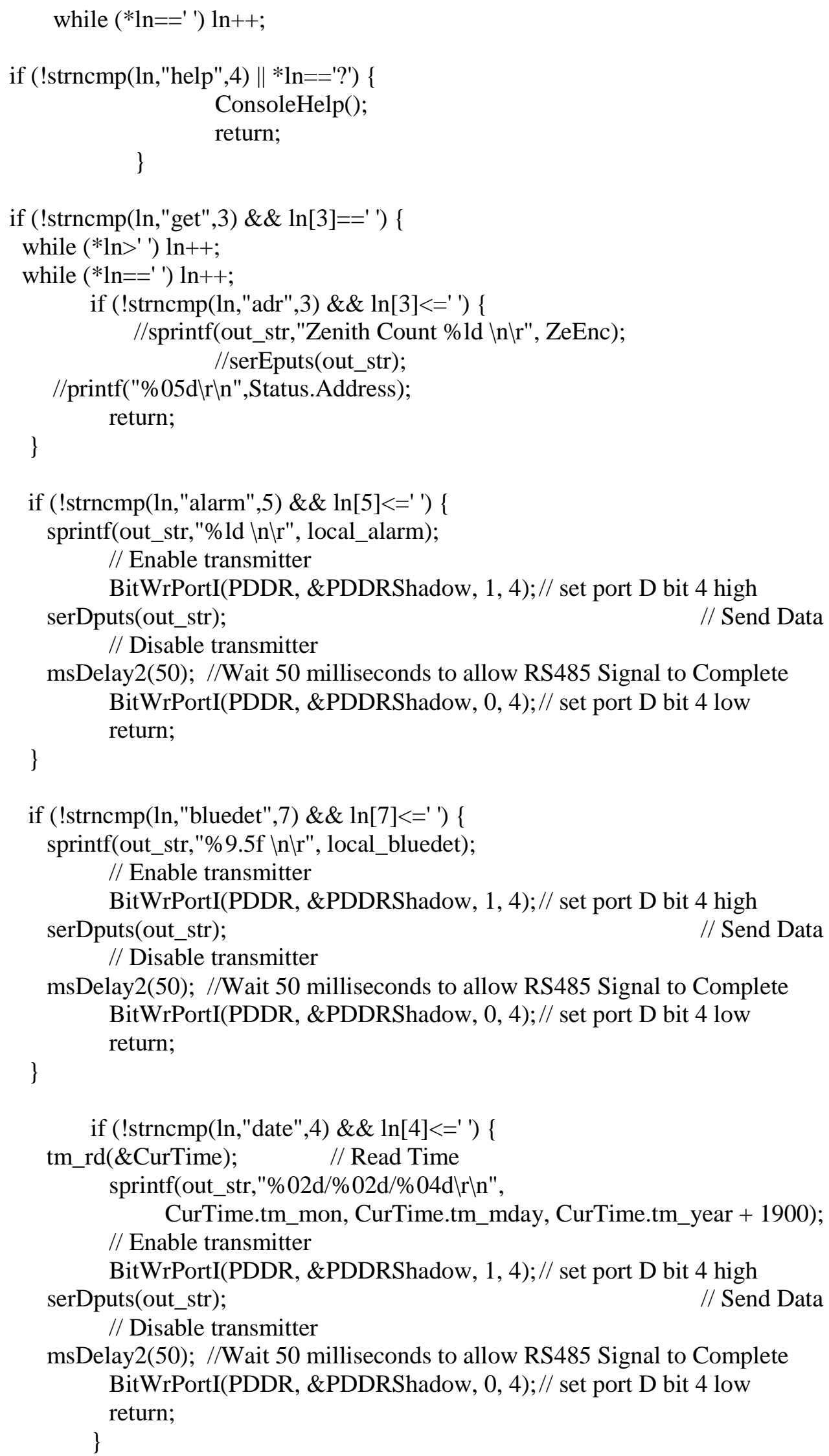




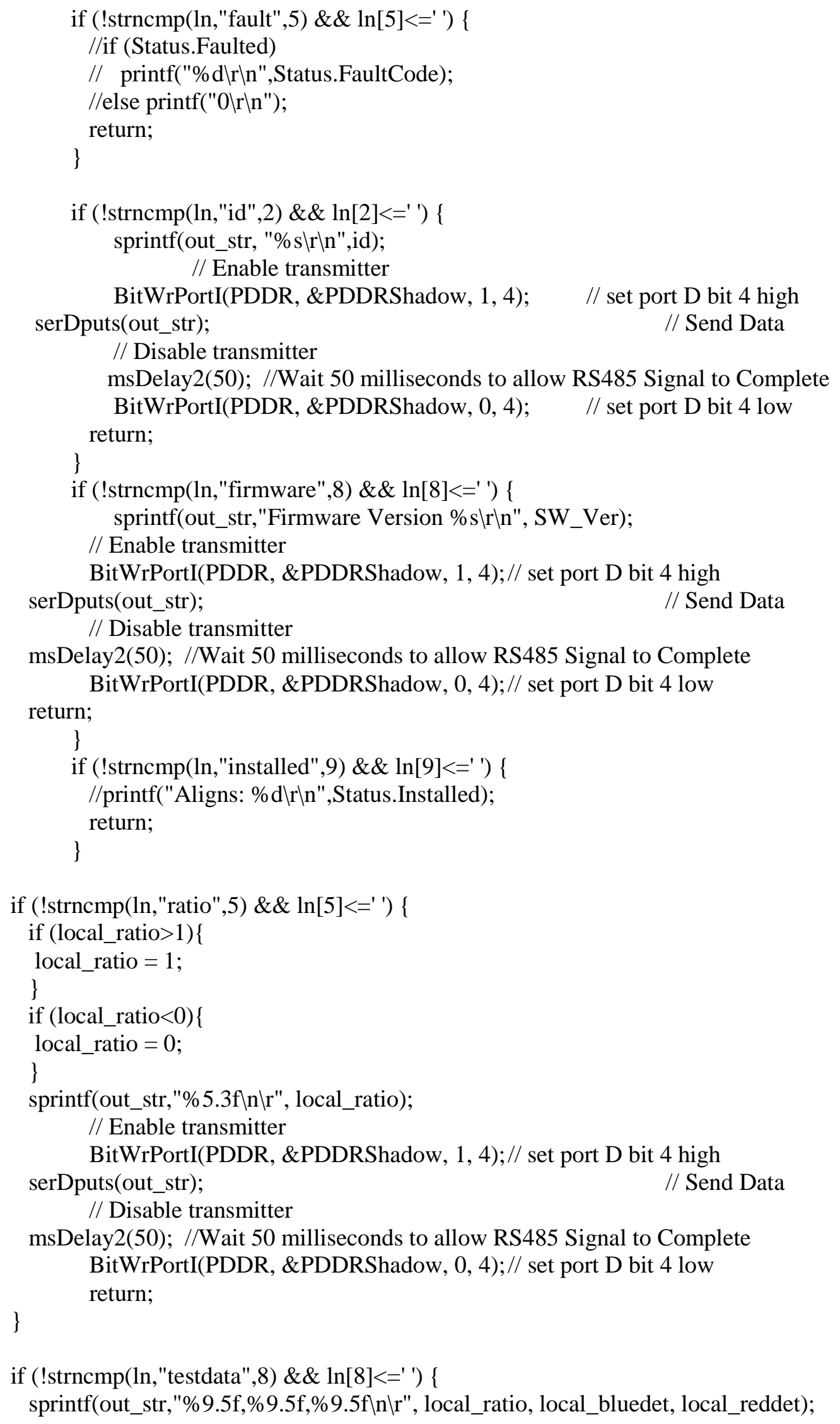


// Enable transmitter

BitWrPortI(PDDR, \&PDDRShadow, 1, 4);// set port D bit 4 high serDputs(out_str);

// Send Data

// Disable transmitter

msDelay2(50); //Wait 50 milliseconds to allow RS485 Signal to Complete BitWrPortI(PDDR, \&PDDRShadow, 0, 4); // set port D bit 4 low

\} return;

if (!strncmp(ln,"reddet",6) \&\& $\ln [6]<==^{\prime}$ ') \{

sprintf(out_str,"\%9.5f $\backslash n \backslash r "$, local_reddet);

// Enable transmitter

BitWrPortI(PDDR, \&PDDRShadow, 1, 4); // set port D bit 4 high serDputs(out_str);

// Disable transmitter

msDelay2(50); //Wait 50 milliseconds to allow RS485 Signal to Complete

BitWrPortI(PDDR, \&PDDRShadow, 0, 4); // set port D bit 4 low

return;

\}

if (!strncmp(ln,"sernum",6) \&\& $\ln [6]<=$ ' ') \{

sprintf(out_str,"\%5dırln",SerialNumber);

// Enable transmitter

BitWrPortI(PDDR, \&PDDRShadow, 1, 4);// set port D bit 4 high

serDputs(out_str); / Send Data

// Disable transmitter

msDelay2(50); //Wait 50 milliseconds to allow RS485 Signal to Complete

BitWrPortI(PDDR, \&PDDRShadow, 0, 4);// set port D bit 4 low

return;

\}

if (!strncmp(ln,"ut",2) \&\& $\ln [2]<=$ ' ') \{

tm_rd(\&CurTime); // Read Time

sprintf(out_str,"\%02d:\%02d:\%02d \rın",CurTime.tm_hour, CurTime.tm_min,

CurTime.tm_sec );

// Enable transmitter

BitWrPortI(PDDR, \&PDDRShadow, 1, 4);// set port D bit 4 high serDputs(out_str);

// Send Data

// Disable transmitter

msDelay2(50); //Wait 50 milliseconds to allow RS485 Signal to Complete BitWrPortI(PDDR, \&PDDRShadow, 0, 4); // set port D bit 4 low return;

\}

\}

if (!strncmp $(\ln , "$ set",3) \&\& $\ln [3]=='$ ') \{

while $(* \ln >$ ' ') $\ln ++$;

while $\left(* \ln ===^{\prime}\right) \ln ++$;

//if (!strncmp(ln,"adr",3) \&\& $\ln [3]=='$ ') \{

$/ /$ while $(* \ln >$ ' ') $\ln ++$;

// while $(* \ln =='$ ') $\ln ++$;

// sscanf(ln,"\%d",\&Status.Address); 


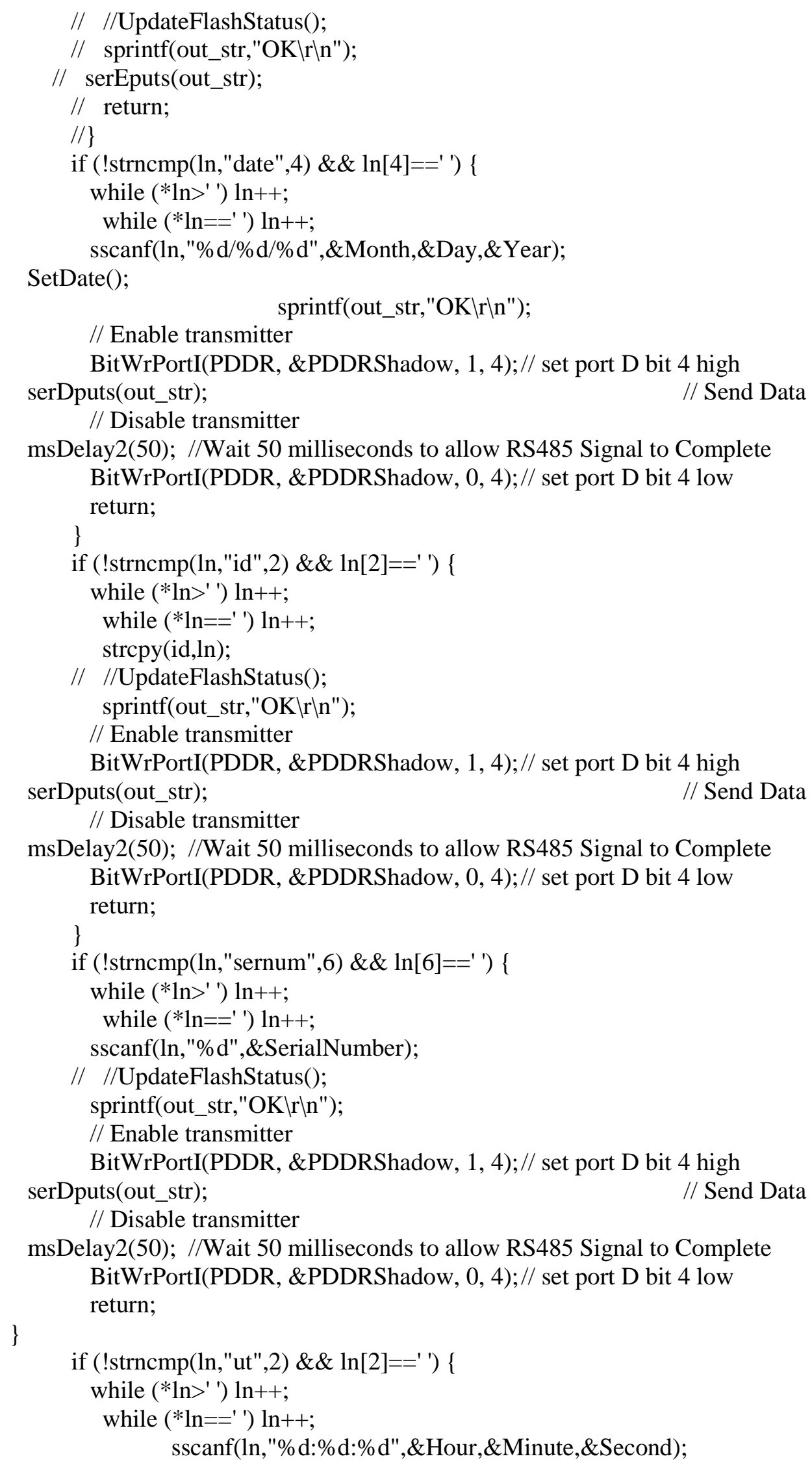




\section{// //UpdateFlashStatus(); \\ SetDate(); \\ sprintf(out_str,"OK\r\n"); \\ // Enable transmitter}

BitWrPortI(PDDR, \&PDDRShadow, 1, 4); // set port D bit 4 high

serDputs(out_str); // Send Data

// Disable transmitter

msDelay2(50); //Wait 50 milliseconds to allow RS485 Signal to Complete

BitWrPortI(PDDR, \&PDDRShadow, 0, 4); // set port D bit 4 low

\} return;

\} 


\title{
APPENDIX F. FUEL MARKER SENSOR COMMAND SPECIFICATION
}

\author{
Oak Ridge National Laboratory \\ Fuel Marker Sensor Command Specification \\ Firmware Version 0.4
}

$01 / 21 / 2010$

Document Version 1.4

\section{INTRODUCTION}

This document describes the RS485 command set used with the ORNL Fuel Marker sensor. The communication protocol is designed so that multiple sensors may be commanded and controlled by either a knowledgeable operator with an ASCII terminal device, or via simple control hardware that communicates using the protocol described in this document.

\section{PHYSICAL LAYER}

All communications employ a 3-wire, half-duplex, RS485 physical connection. Communication on the RS485 is conducted using standard asynchronous start stop protocol at 19200 baud, using 1 stop bit and no parity.

\section{COMMAND PROTOCOL}

The following sections outline the master command syntax and summarize the action of units to the commands. In general, all commands follow the same ASCII format shown below:

$$
\text { \$ + COMPARTMENT_IDENTIFIER + Command + Carriage Return (ASCII code 0x0D). }
$$

There are five COMPARTMENT_IDENTIFIER that identify the position of a given sensor in a fuel compartment on a tanker vehicle. They include:

\begin{tabular}{|c|c|}
\hline COMPARTMENT_IDENTIFIER & Sensor Position \\
\hline 100 & Compartment \#1 (Nearest Tanker Cab) \\
\hline 200 & Compartment \#2 \\
\hline 300 & Compartment \#3 \\
\hline 400 & Compartment \#4 \\
\hline 500 & Compartment \#5 (Furthest from Tanker Cab) \\
\hline
\end{tabular}

An example of a command sent to a sensor attached to compartment \#2 would be: \$200help<cr>

This format eliminates cross talk among the various sensors. Reply from the sensors will vary, but most will be composed of one or more lines of text terminated by a line feed and carriage return (ASCII 0x0A, 0x0D). 


\section{Point-to-Point Commands}

\section{\$<COMPARTMENT_IDENTIFIER $>$ get bluedet $<$ cr $>$}

Reply:

ddd.ddd $<$ cr $><$ lf $>$

Summary

The connected unit returns a decimal value of the measured amount of blue fluorescent light measured with the sensor's blue filtered detector.

\section{\$<COMPARTMENT_IDENTIFIER $>$ get date<cr $>$}

Reply:

$$
\mathrm{mm} / \mathrm{dd} / \text { yyyy }<\mathrm{cr}><\mathrm{lf}>
$$

Summary

The connected unit shall return its current UT date.

\section{\$<COMPARTMENT_IDENTIFIER $>$ help $<$ cr $>$}

Reply:

Text list

Summary

Shows help screen.

\section{\$<COMPARTMENT_IDENTIFIER $>$ get id $<$ cr $>$}

Reply:

$$
<\text { adr }>\text { : }<\text { serialum }>-<\text { id }><\text { cr }><\text { lf }>
$$

Summary

Returns a string containing the units address serial number and identity string.

\section{\$<COMPARTMENT_IDENTIFIER > get ratio<cr>}

Reply:

$$
\text { d.ddd }<\text { lf }><\text { cr }>
$$

\section{Summary}

The connected unit returns a decimal value of the measured "ratio" between the amount of fluorescing blue light versus red light measured with the sensor's two filtered detectors. 


\section{\$<COMPARTMENT_IDENTIFIER $>$ get reddet $<$ cr $>$}

Reply:

ddd.ddd $<$ cr $><$ lf $>$

Summary

The connected unit returns a decimal value of the measured amount of red fluorescent light measured with the sensor's red filtered detector.

\section{\$<COMPARTMENT_IDENTIFIER > get sernum<cr $>$}

Reply:

dddddd $<$ cr $><$ lf $>$

Summary

The connected unit shall return its serial number.

\section{\$<COMPARTMENT_IDENTIFIER $>$ get ut $<$ cr $>$}

Reply:

hh:mm:ss.sss $<$ cr $><$ lf $>$

Summary

The connected unit shall return its current internal time.

\section{\$ $<$ COMPARTMENT_IDENTIFIER $>$ set id " $<$ id $>$ " $<$ cr $>$}

Reply:

$$
\mathrm{OK}<\mathrm{cr}><\mathrm{lf}>
$$

Summary

The connected unit will store the id string as its new name. This is the string it will use to reply to connection requests and during roll calls. This string cannot exceed 32 characters.

\section{\$ $<$ COMPARTMENT_IDENTIFIER $>$ set serialnum $<$ dddddd $><$ cr $>$}

Reply:

$$
\mathrm{OK}<\mathrm{cr}><\mathrm{lf}>
$$

Summary

The selected unit's serial number shall be set to the supplied number. The number must be within the range $1 . . .65535$. 
SAMPLE CONFIGURATION SCRIPT

Typical Script for Communicating with Single Sensor (for sensor on compartment 3)

*Commands in Bold

*Responses in Italics

\$300get sernum

1001

\$300get id

Sample Sensor ID

\$300get ratio

1.123

*Ratio value changes with increase or decrease in fuel marker concentrations 
APPENDIX G. CUSTOM SIGHT GLASS DRAWINGS

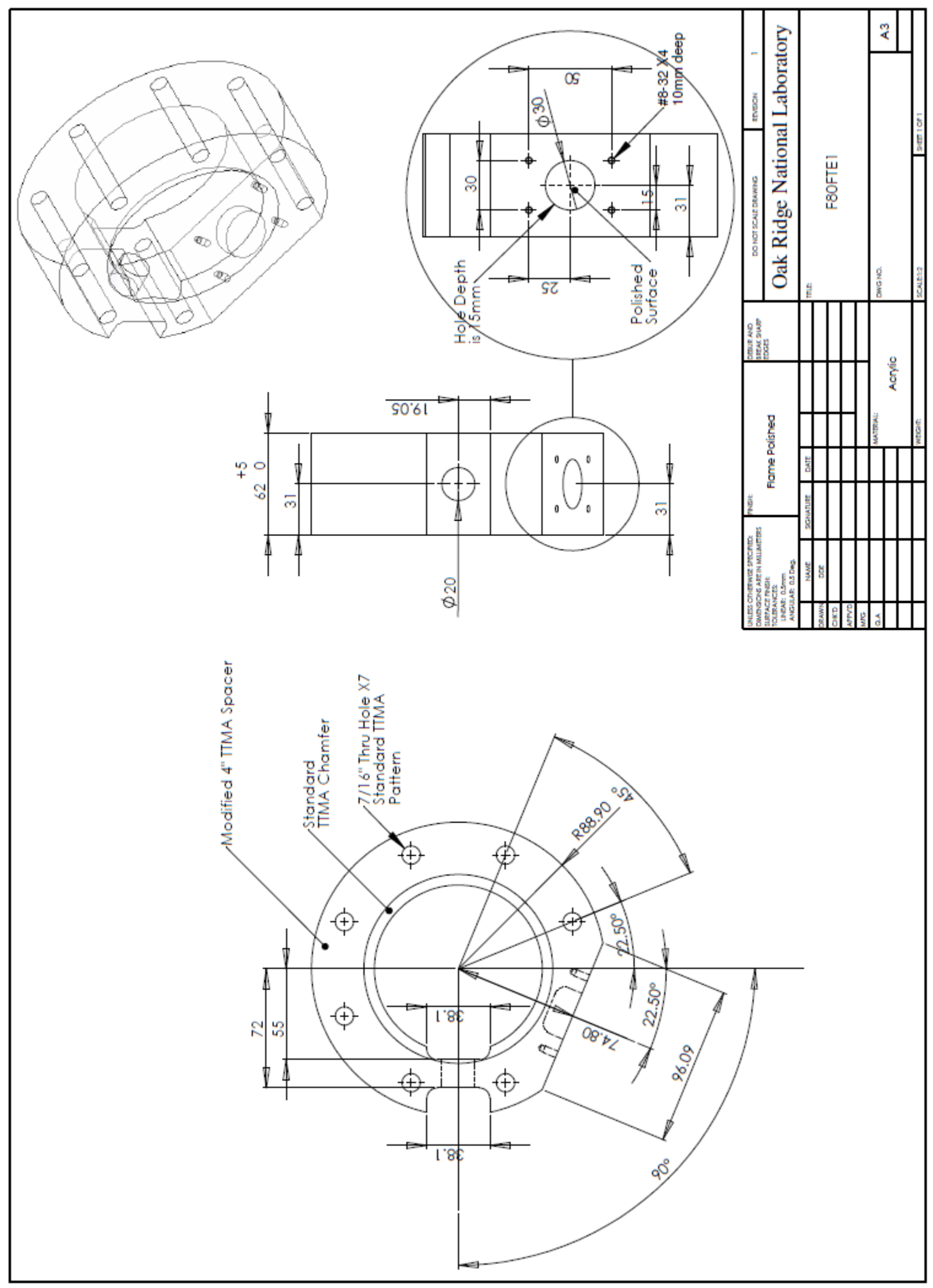





\begin{tabular}{|c|l|c|c|}
\hline \multicolumn{5}{|c|}{ RTDBA POC Final Report Revision Log } \\
\hline $\begin{array}{c}\text { Revision } \\
\text { Number }\end{array}$ & \multicolumn{1}{|c|}{ Description of Change } & Editor(s) & $\begin{array}{c}\text { Change Effective } \\
\text { Date }\end{array}$ \\
\hline 0 & Initial outline & MBL & 05 May 2011 \\
\hline 1 & Draft Chapter 1 & SAM & 18 May 2011 \\
\hline 2 & Full report & SAM & 19 Aug 2011 \\
\hline 3 & $\begin{array}{l}\text { Added recommended architecture and } \\
\text { cost-effectiveness evaluation }\end{array}$ & MBL & 26 Sep 2011 \\
\hline & & & \\
\hline
\end{tabular}

